\title{
Álgebra Homológica em Topos
}

\author{
Ana Luiza da Conceição Tenório \\ DISSERTAÇÃO APRESENTADA \\ $\mathrm{AO}$ \\ Instituto De MATEMÁticA E EstatísticA \\ $\mathrm{DA}$ \\ Universidade DE SÃo PaUlo \\ PARA \\ OBTENÇÃO DO TÍTULO \\ $\mathrm{DE}$ \\ Mestre em CiÊnCIAS \\ Programa: Matemática \\ Orientador: Prof. Dr. Hugo Luiz Mariano
}

Durante o desenvolvimento deste trabalho o autor recebeu auxílio financeiro do $\mathrm{CNPq}$

São Paulo, março de 2019 


\section{Álgebra Homológica em Topos}

Esta versão da dissertação contém as correções e alterações sugeridas pela Comissão Julgadora durante a defesa da versão original do trabalho, realizada em 19/2/2019. Uma cópia da versão original está disponível no Instituto de Matemática e Estatística da Universidade de São Paulo.

Comissão Julgadora:

- Prof. Dr. Prof. Dr. Hugo Luiz Mariano (orientador) - IME-USP

- Prof. Dr. Walter Alberto de Siqueira Pedra - IF-USP

- Prof. Dr. Peter Arndt - Universidade de Düsseldorf 


\section{Agradecimentos}

Os anos dedicados ao Mestrados foram muito importantes e gratificantes para mim. Sei que o trabalho só pode ser desenvolvido graças a uma série de situações e pessoas que contribuíram e muito para minha formação em Matemática e como pessoa, a elas tento demonstrar minha gratidão cotidianamente.

Contudo, algumas pessoas estiveram mais diretamente envolvidos com a Dissertação. Agradeço ao meu orientador, Hugo Luiz Mariano, por todo o tempo dedicado, pela atenção e pelo cuidado durante os seminários, correções de texto, conversas sobre Matemática e todas as atividades que a Pós-Graduação demanda. Poder ter liberdade para seguir esse tema de pesquisa e ser orientada com tanto afinco foi uma grande oportunidade.

Aos professores Peter Arndt e Walter Pedra, sou grata pelos comentários que não apenas enriquecem este texto, como também apresentam novas possibilidades de pesquisa.

Deixo também meus agradecimento aos coletivos Existimos! e Mulheres do IME. Ter mulheres - alunas e professoras - nas quais pude me inspirar foi fundamental nesse percurso. Agradeço também aquelas que pelos mais diversos motivos não participam dos coletivos, mas contribuiram para a minha formação - são todas muito queridas.

Aos colegas com quem cursei disciplinas, obrigada. A Pós-Graduação tem suas dificuldades, mas podemos compartilhá-las e tornar esse um momento a ser lembrado com carinho.

Agradeço a minha família pelo suporte constante, mesmo que a realidade da carreira acadêmica seja distante da que vocês conhecem, minha escolha foi sempre respeitada e acolhida.

Por fim, agreadeço ao CNPq, Conselho Nacional de Desenvolvimento Científico e Tecnológico, pelo apoio fornecido, sem o qual esse trabalho não teria sido possível. 


\section{Resumo}

TENÓRIO, A. L. C. Álgebra Homológica em Topos. 2019. 129 f. Dissertação (Mestrado) - Instituto de Matemática e Estatística, Universidade de São Paulo, São Paulo, 2019.

O objetivo dessa Dissertação é detalhar resultados conhecidos de Cohomologia em Topos de Grothendieck. Para isso, apresentamos a Álgebra Homológica em seu contexto mais geral, através de Categorias Abelianas, introduzindo as principais noções da área como funtores derivados e sequências espectrais. Desenvolvemos também o essencial da Teoria de Topos, explicando como um topos de Grothendieck surge como uma certa generalização dos feixes de conjuntos e fornecemos aspectos lógicos dos topos elementares. Focamos sobretudo nos Topos de Grothendieck pois a partir deles podemos construir categorias abelianas com suficientes injetivos, as quais são necessárias para expressar os grupos de cohomologia.

Palavras-chave: Categorias Abelianas, Álgebra Homológica, Feixes, Topos de Grothendieck. 


\section{Abstract}

TENÓRIO, A. L. C. Homological Algebra in Toposes. 2019. 129 f. Dissertação (Mestrado) - Instituto de Matemática e Estatística, Universidade de São Paulo, São Paulo, 2019.

The final objective of this Dissertation is to detail known results of Cohomology in Grothendieck Topos. For this, we present Homological Algebra in its more general context, through Abelian Categories, introducing the main notions of the area as derived functors and spectral sequences. We also develop the basics of the Topos Theory, explaining how a Grothendieck Topos arises as a certain generalization of sheafs and we provide logical aspects of the elementary topos. We focus mainly in the Grothendieck Topos because from them we can construct abelians categories with sufficient injectives, which are necessary to express the cohomology groups.

Keywords: Abelian Categories, Homological Algebra, Sheaves, Grothendieck Topos. 


\section{Sumário}

Introdução $\quad$ ix

1 Categorias Abelianas e Álgebra Homológica $\quad 1$

1.1 Categorias Abelianas . . . . . . . . . . . . . . . . . . . . 1

1.2 Funtores Exatos . . . . . . . . . . . . . . . . . . . . . . . 13

1.3 Homologia e Cohomologia . . . . . . . . . . . . . . . . . . 16

1.4 Funtores Derivados . . . . . . . . . . . . . . . . . . 20

1.5 Funtor Ext . . . . . . . . . . . . . . . . . . . . 31

1.6 Sequências Espectrais . . . . . . . . . . . . . . . . . . 34

1.7 Complementos sobre Categorias Abelianas . . . . . . . . . . . . . . . 36

2 Teoria de Topos $\quad 43$

2.1 Feixes de Conjuntos . . . . . . . . . . . . . . . . . . . . . 44

2.2 Topologias e Topos de Grothendieck e Feixes . . . . . . . . . . . . . . . 50

2.3 Topos Elementares . . . . . . . . . . . . . . . . . . . . . . . . . 61

2.4 Morfismos Geométricos e Categoria Slice . . . . . . . . . . . . . . . . . . 70

2.5 Álgebras de Heyting em um Topos . . . . . . . . . . . . . . . . . . . . . . . 79

2.6 Aspectos Lógicos da Teoria de Topos . . . . . . . . . . . . . . . . . . . . 87

3 Álgebra Homológica em Topos $\quad 95$

3.1 Feixes Algébricos . . . . . . . . . . . . . . . . . . . . . . 95

3.2 Cohomologia de Feixes . . . . . . . . . . . . . . . . . . 100

3.3 Cohomologia de $\mathbf{A b}(\mathcal{E}) \ldots \ldots \ldots . \ldots \ldots$

3.4 Cohomologia de Čech . . . . . . . . . . . . . . . . . . . . 112

4 Considerações Finais $\quad 125$

5 Apêndice $\quad 129$

$\begin{array}{ll}\text { Referências Bibliográficas } & 131\end{array}$ 


\section{Introdução}

A Matemática está em constante desenvolvimento. Grandes áreas de estudo do presente, no passado, atuaram como ferramentas capazes meramente de auxiliar teorias em foco, mas muitas dessas cresceram e agora caminham de forma independente. Dois bons exemplos desse fenômeno são a Álgebra Homológica e a Teoria de Topos.

A princípio, Álgebra Homológica era uma linguagem especialmente útil à Topologia Algébrica para fins computacionais envolvendo grupos de homologia, além de ter sido anteriormente utilizada no século XIX por B. Riemann e E. Betti em "números de homologia". Contudo, por volta de 1940 o estudo dos funtores Ext e Tor, entre outros objetos, ampliou a atuação da álgebra homológica culminando no aparecimento do clássico: Homological Algebra de H. Cartan e S. Eilenberg [CE99]. Através dessa obra a teoria foi consolidada e marcou o seu primeiro grande período, com homologias e cohomologias de grupos e álgebras de Lie, sequências espectrais e cohomologia feixes, por exemplo.

O segundo período inicia-se com o famoso "Tohoku", artigo de A. Grothendieck cujo nome oficial é Sur quelques points d'algèbre homologique [Gro57]. Aqui a noção de categoria derivada é desenvolvida graças aos métodos cohomológicos; surgem os $\delta$-funtores, a cohomologia de feixes generalizados e a étale (esta última responsável pela demonstração de Deligne das Conjecturas de Weil). Além disso a introdução de categorias abelianas levou a uma formulação da álgebra homológica onde não era mais necessário distinguir entre feixes de grupos abelianos e módulos sobre um anel. Vemos, portanto, que este foi um momento bastante frutífero tanto para Álgebra Homológica quanto para a Geometria Algébrica.

$\mathrm{O}$ terceiro período diz respeito às pesquisa atuais na área, onde o uso de categorias derivadas e as trianguladas crescem cada vez mais, ampliando sua utilidade para novas áreas tais como a teoria de $D$-módulos.

Agora, para falarmos da teoria de topos é mais interessante fazermos uma divisão baseada nas abordagens em vez dos períodos, ou seja, podemos nos aproximar do que é um topos partindo de pontos muitos distintos e visualizar de forma diferente um mesmo objeto. Todavia, vamos assinalar apenas as duas primeiras abordagens que surgiram, quase simultaneamente: via a Geometria Algébrica de Grothendieck e via Lógica Categórica de W. Lawvere.

Na primeira metade do século XX um problema conhecido da Geometria Algébrica era que a topologia de Zariski das variedades algébricas não possuia "a quantidade suficiente de abertos" para produzir uma noção adequada de localização do ponto de vista geométrico. Nos anos 60 Grothendieck considerou introduzir "morfismos étale" no lugar de "inclusão por abertos de Zariski", mas para isso foi necessário generalizar a noção de feixes para um novo contexto de topologia, a de Grothendieck. Assim formou-se um plano de fundo para 
o nascimento da cohomologia étale.

Entre os resultados que surgiram na época encontra-se o Teorema de Giraud, fornecendo as equivalências que definem um topos (de Grothendieck). É com esse teorema que topos passou a ser sinônimo de um "espaço generalizado", pois uma das equivalências diz que um topos é uma categoria de feixes munido da topologia de Grothendieck e uma categoria com uma topologia pode ser vista como um espaço topológico generalizado. Nesse contexto os topos são associados a ferramentas para o desenvolvimento de diversas teorias de cohomologia, não só da étale.

A outra abordagem que deu origem ao conceito de topos vem do trabalho de Lawvere em teoria das categorias e permite uma definição mais geral, na qual existem topos que não são topos de Grothendieck. Seu uso tem sido principalmente em áreas ligadas à Lógica.

Historicamente é importante dizer que a primeira tentativa de definir topos nessa direção não foi bem sucedida, pois era especializada demais e não dialogava com outras teorias. Apenas quando Lawvere voltou sua atenção para os topos de Grothendieck e percebeu que eles possuem um objeto $\Omega$ de valores de verdade é que foi acrescido na definição de topos a noção do classificador de subobjetos $\Omega$ e então a teoria deslanchou e ficou conhecida como "teoria elementar de topos".

Atualmente as duas abordagens que citamos têm deixado sua marca. A primeira continua sendo utilizada nas pesquisas em Geometria Algébrica devido ao seu papel em cohomologias e a segunda tem sido aplicada, por exemplo, em Teoria de Homotopia (classificação de topos) e também Ciência da Computação Teórica.

Outra visão moderna e a qual seguiremos nesse projeto é a dos toposes como uma teo$\mathrm{ria}^{1}$, mais precisamente como uma teoria cohomológica. Usaremos a linguagem de Álgebra Homológica do terceiro período, mas teremos sempre em mente a bagagem histórica dos anteriores, consultando e citando os grandes clássicos mencionados acima.

Segue uma breve descrição dos capítulos dessa Dissertação.

Começamos o Capítulo 1 falando sobre categorias abelianas. É comum nos livros de Álgebra Homológica desenvolverem a teoria na categoria $R$-Mod, que é um exemplo categoria abeliana, mas pouco se fala sobre as modificações que seriam necessárias no contexto mais geral. Usamos [Bor94b] para apresentar o básico categorias abelianas, posteriormente, nas seções 3, 4, 5 e 6 introduzimos os conceitos e resultados mais conhecidos de Álgebra Homológica tendo como principal referência [Wei95] 3, 4, 5 e 6, mas pontuando as diferenças de estar em categorias abelianas em vez de $R$-Mod. Na última seção voltamos a nos guiar por [Bor94b] para demonstrar o Lema da Serpente usando os chamados "pseudoelementos" e justificar adequadamente porquê os resultados de Álgebra Homológica valem para categorias abelianas.

No Capítulo 2 esquecemos de Álgebra Homológica começamos a falar da Teoria de Topos, o conteúdo apresentado está essencialmente em [Joh14] e [MLM94]. Iniciamos com feixes de conjuntos para motivar a definição dos Topos de Grothendieck na seção seguinte, onde estudamos propriedades do funtor $a: \operatorname{Sets}^{\mathcal{C}^{o p}} \rightarrow \operatorname{Sh}(\mathcal{C}, J)$ da categoria dos pré-feixes em um feixe munido com uma topologia de Grothendieck $J$. Na seção 3 focamos nos topos elementares, um conceito mais geral que Topos de Grothendieck, e ao estudar sua

\footnotetext{
${ }^{1}$ No livro Sketches of an Elephant: A Topos Theory Compendium de Peter T. Johnstone, os topos são vistos de várias perspectivas - como categorias, espaços, teorias e universos matemáticos - é no mesmo sentido do que o utilizado nessa obra que dizemos "topos como teoria".
} 
estrutura nos deparamos com vários resultados que categorias abelianas também possuem. As seções seguintes nos fornecem mais informação sobre o topos elementares. Morfismos geométricos e a categoria slice serão bastante utilizados no Capítulo seguinte, a seção 5 não é diretamente utilizada, mas induz a pensarmos em topos de um ponto de vista da Lógica depois de termos percorrido um caminho geométrico através dos feixes e fortalecemos essa outra perspectiva com a última seção.

O Capítulo 3 tem a função de reunir os dois capítulos anteriores, o que fazemos nas seções 3 e 4 onde aplicamos Álgebra Homológica em um Topos de Grothendieck. A Cohomologia de feixes é bem conhecida e nos aproveitamos dela para motivar a Cohomologia em um topos de Grothendieck, umas vez que este é, de certa forma, uma generalização de feixes. Um conceito fundamental dessa parte é a categoria $\mathbf{A b}(\mathcal{E})$ de objeto de grupo abeliano de $\mathcal{E}=S h(\mathcal{C}, J)$, pois é uma categoria abeliana que possui suficientes injetivos e é através delas que definiremos os objetos de cohomologia de um Topos de Grothendieck.

A Dissertação é concluída no Capítulo 4 comparando categorias abelianas com topos e cohomologia de feixes com cohomologia de um Topos de Grothendieck além de deixarmos caminhos para pesquisas futuras.

Ao longo do texto assumimos que o eventual leitor tem familiariedade com conhecimentos básicos de teoria de categorias. 


\section{Capítulo 1}

\section{Categorias Abelianas e Álgebra Homológica}

O primeiro grande marco da Álgebra Homológica foi a publicação do livro "Homological Algebra"de H. Cartan e S. Eilenberg, em 1956 ([CE99]). Muitas das ideias presentes do livros como complexos de cadeia e resoluções já estavam circulando no meio matemático, mas foi nessa obra que esses conceitos foram agrupados pela primeira vez e apareceram definições inéditas como módulos projetivos e funtores derivados. As construções foram todas feitas para $R$-módulos mas eram evidentes os paralelos com o que já tinha sido feito até então para cohomoliga de feixes, portanto era natural querer encontrar um ambiente mais geral para desenvolver a Álgebra Homológica de forma que englobasse módulos e feixes simultâneamente. Quem teve a abordagem mais bem sucedida nesse aspecto foi A. Grothendieck em 1957 [Gro57]. Nesse artigo desenvolve-se Álgebra Homológica em Categorias Abelianas e mais generalizações são possíveis: é o caso dos $\delta$-funtores em relação aos funtores derivados e a sequência espectral de Grothendieck em relação à sequência espectral de Leray.

Todavia, também surgiram alguns problemas. Em categorias abelianas a noção de elemento se perde e a princípio não faria sentido procurar elementos através de caça ao diagramas, técnica que permite demonstrar resultados conhecidos como o Lema da Serpente. Uma forma de resolver essa questão é através do resultado de P. Freyd que diz que algumas categorias abelianas podem ser mergulhadas na categoria dos módulos sobre um anel, [Fre64]. Entretanto, nessa Dissertação faremos outra abordagem: introduziremos o conceito de pseudoelemento, como em [Bor94c], também conhecido como elemento generalizado [ML98].

Não temos resultados novos ou demonstrações inovadoras, mas trabalharemos com homologia e cohomologia no ambiente das categorias abelianas, o que mesmo hoje em dia não é tão fácil encontrar.

As referências utilizadas para as seções 1, 2 e 7 desse capítulo foram [Bor94b] e [Fre64]. Nas demais seções utilizamos principalmente [GM02], [Wei95] e [HS71].

Além disso, recomendamos também entitulado "A History of Homological Algebra", disponível no site de Charles A. Weibel, que conta a história da Álgebra Homológica desde quando começou, como mais uma ramificação da Topologia Algébrica, até aplicações modernas em, por exemplo, $C^{*}$-álgebras.

\subsection{Categorias Abelianas}

A cohomologia de feixes e a cohomologia de grupos apresentam semelhanças: de fato, ambas surgem como funtores derivados em categorias abelianas.

Não falaremos sobre cohomologia de grupos, mas no terceiro capítulo iremos trabalhar um pouco com a cohomologia de feixes para motivar o desenvolvimento da cohomologia de topos. Queremos com esse comentário tornar clara a importância das categorias abelianas 
mas nessa seção apenas apresentaremos os principais resultados e definições, seguindo a forma de [Bor94b].

Definição 1.1.1 Seja $\mathscr{C}$ uma categoria.

1. Um objeto $t$ é dito terminal em $\mathscr{C}$, se para todo objeto $a$ em $\mathscr{C}$ existe um único morfismo $a \rightarrow t$.

2. Um objeto $s$ é dito inicial em $\mathscr{C}$, se para todo objeto $a$ em $\mathscr{C}$ existe um único morfismo $s \rightarrow a$.

3. Um objeto em $\mathscr{C}$ é dito nulo (ou zero) se é terminal e inicial simultaneamente.

Observe que dois objetos terminais (ou iniciais) são isomorfos. Se, por exemplo, $t$ é terminal, existe um único morfismo $t \rightarrow t$, o morfismo identidade.

Definição 1.1.2 Seja $\mathscr{C}$ uma categoria com objeto nulo 0 . Um morfismo $f: A \rightarrow B$ é dito nulo quando se fatora ao longo de 0 , isto é, o seguinte diagrama comuta:

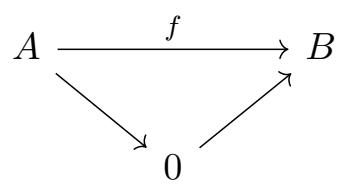

Proposição 1.1.3 Em uma categoria $\mathscr{C}$ com objeto nulo 0 , a composição de um morfismo nulo com um morfismo arbitrário é também um morfismo nulo.

\section{Demonstração:}

Seja $f: A \rightarrow B$ o morfismo nulo e $g: B \rightarrow C$ um morfismo qualquer. Digamos que a fatoração ao longo de 0 é da forma $f=r s, \operatorname{com} r: 0 \rightarrow B$ e $s: A \rightarrow 0$. Então $f g=r s g$, logo, a composição $f g$ se fatora ao longo de 0 também.

É comum denotar tanto o objeto nulo como o morfismo nulo por 0, ficando claro ao que nos referimos pelo contexto.

Definição 1.1.4 Seja $\mathscr{C}$ uma categoria com objeto nulo 0. Um morfismo $k: K \rightarrow A$ é kernel de $f: A \rightarrow B$ se for o equalizador de $f$ e do morfismo nulo $0: A \rightarrow B$. $f \circ k=0$ e para todo morfismo $h$ tal que $f \circ h=0$ existe uma única $h^{\prime}$ tal que $h=k \circ h^{\prime}$.

Podemos reescrever essa definição em termos da propriedade universal do equalizador.

$k: K \rightarrow A$ é kernel de $f: A \rightarrow B$ se $f \circ k=0$ e para todo morfismo $h$ tal que $f \circ h=0$ existe uma única $h^{\prime}$ tal que $h=k \circ h^{\prime}$. Em forma de diagrama temos:

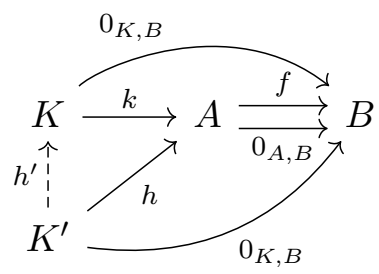

Sabendo que um equalizador é um monomorfismo (Teorema 2.6 [McL96]) imediatamente obtemos que o kernel é mono. Analogamente, o cokernel - definido de forma dual é um epirmorfismo. Não é difícil mostrar que, se existirem, tanto o kernel como o cokernel são único a menos de isomorfismo.

Vamos provar pequenos resultados com essas definições para nos acostumarmos.

Proposição 1.1.5 Seja $f$ um monomorfismo em uma categoria com morfismos nulos. Se $f \circ g=0$, para algum morfismo $g$, então $g=0$. 


\section{Demonstração:}

Pela Proposição 1.1.4 ao compor $f$ com o morfismo nulo temos $f \circ 0=0$. Como $f \circ g=0$, temos $f \circ g=f \circ 0$ que implica $g=0$ pois $f$ é mono.

Proposição 1.1.6 Numa categoria com morfismos nulos o kernel de morfismo nulo 0 : $A \rightarrow B$ é, a menos de isomorfismo, a identidade em $A$.

\section{Demonstração:}

Temos $0 \circ i d_{A}=0$ e $0 \circ 0=g$, dado $g: X \rightarrow A$ qualquer, ambas igualdades valem pela Proposição 1.1.4. Então $i d_{A}$ equaliza 0, pois $g$ se fatora ao longo de $i d_{A}$ apenas pelo próprio morfismo $g$, isto é, $g$ é único tal que $g=i d_{A} \circ g$.

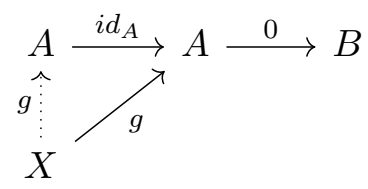

Com esse conceitos básicos introduzidos podemos dar início ao nosso caminho em direção às categorias abelianas, primeiro percorrendo as noções de categorias pré-aditivas e categorias aditivas.

Definição 1.1.7 Uma categoria $\mathscr{C}$ é dita pré-aditiva (ou Ab-categoria) se cada conjunto $\operatorname{Hom}(A, B)$, com $A$ e $B$ objetos de $\mathscr{C}$, possui estrutura de um grupo abeliano e a composição dos morfismos é bilinear, da seguinte forma:

$$
\begin{gathered}
\operatorname{Hom}(A, B) \times \operatorname{Hom}(B, C) \rightarrow \operatorname{Hom}(A, C),(f, g) \mapsto g \circ f \\
\left(g+g^{\prime}\right) \circ\left(f+f^{\prime}\right)=g \circ f+g \circ f^{\prime}+g^{\prime} \circ f+g^{\prime} \circ f^{\prime}
\end{gathered}
$$

As categorias dos grupos abelianos (Ab) e dos módulos sobre aneis (R-Mod e Mod-R) são exemplos de categorias pré-aditivas.

Temos um lema que nos será útil.

Lema 1.1.8 Sejam $A, B$ e $C$ objetos de uma categoria pré-aditiva $\mathscr{C}$. Se $f$ é o zero de $\operatorname{Hom}(A, B)$ ou se g é o zero de $\operatorname{Hom}(B, C)$, então $g \circ f$ é zero de $\operatorname{Hom}(A, C)$.

\section{Demonstração:}

Se $g$ é zero de $\operatorname{Hom}(B, C)$, usando a bilinearidade temos $g \circ f+g \circ f=(g+g) \circ f=g \circ f$, $\log$, $g \circ f=0$. Procedemos analogamente se $f$ é zero de $\operatorname{Hom}(A, B)$.

Proposição 1.1.9 Seja $\mathscr{C}$ categoria pré-aditiva. São equivalentes:

(i) $\mathscr{C}$ possui objeto inicial.

(ii) $\mathscr{C}$ possui objeto terminal.

(iii) $\mathscr{C}$ possui objeto nulo.

\section{Demonstração:}

Pela definição de objeto nulo é imediato que (iii) implica $(i)$ e $(i i)$. Vamos mostrar apenas que $(i)$ implica (iii), pois demonstração de $(i i) \Longrightarrow$ (iii) é dual, basicamente, bastaria inverter o sentido das flechas.

Seja 0 objeto inicial, como 0 é inicial existe um único morfismo que vai de 0 em 0 , a identidade. Então $i d_{0}$ é o único elemento de $\operatorname{Hom}(0,0)$.

Se tomarmos $C$ objeto de $\mathscr{C}$, teremos que o grupo $\operatorname{Hom}(C, 0)$ possui pelo menos um elemento, a saber, o zero. Mas veja que se $f: C \rightarrow 0$ é um morfismo qualquer, fazemos $\operatorname{Hom}(C, 0) \times \operatorname{Hom}(0,0) \rightarrow \operatorname{Hom}(C, 0),\left(f, i d_{0}\right) \mapsto i d_{0} \circ f=f$ e obtemos que $f$ é o zero de $\operatorname{Hom}(C, 0)$, pois $i d_{0}$ é o zero de $\operatorname{Hom}(0,0)$ e aplicamos o Lema 1.1.8. Isso vale para qualquer objeto $C$, logo, 0 é também terminal e, portanto, nulo. 
Proposição 1.1.10 Dados $A, B$ objetos em uma categoria pré-aditiva $\mathscr{C}$. São equivalentes:

(i) O produto $\left(P, p_{A}, p_{B}\right)$ de $A$ e $B$ existe.

(ii) $O$ coproduto $\left(P, s_{A}, s_{B}\right)$ de $A$ e $B$ existe.

(iii) Existe um objeto $P$ e morfismos

$$
p_{A}: P \rightarrow A, p_{B}: P \rightarrow B, s_{A}: A \rightarrow P \text { e } s_{B}: B \rightarrow P \text { com as propriedades }
$$
$p_{A} \circ s_{A}=i d_{A}, p_{B} \circ s_{B}=i d_{B}, p_{A} \circ s_{B}=0, p_{B} \circ s_{A}=0 e s_{A} \circ p_{A}+s_{B} \circ p_{B}=i d_{P}$

Nessas condições ainda temos

$$
s_{A}=\operatorname{ker}\left(p_{B}\right), s_{B}=\operatorname{ker}\left(p_{A}\right), p_{A}=\operatorname{coker}\left(s_{B}\right), p_{B}=\operatorname{coker}\left(s_{A}\right)
$$

\section{Demonstração:}

Mais uma vez por dualidade, é suficiente mostrar $(i) \Longleftrightarrow($ iii $)$.

$((i) \Longrightarrow($ iii $))$ As primeiras propriedades são imediatas da definição de produto. Dados $A$ e $B$ cujo produto é $P$ e $p_{A}$ e $p_{B}$ são as projeções, definimos $s_{A}: A \rightarrow P$ como o único morfismo tal que $p_{A} \circ s_{A}=i d_{A}$ e $p_{B} \circ s_{A}=0$, isto é, o diagrama abaixo comuta

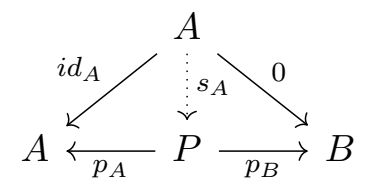

Analogamente definimos $s_{B}: B \rightarrow P$.

Agora fazemos algumas contas, compondo $p_{A}$ e $p_{B}$ com a soma da última propriedade que queremos provar:

(I) $p_{A} \circ\left(s_{A} \circ p_{A}+s_{B} \circ p_{B}\right)=\left(p_{A} \circ s_{A}\right) \circ p_{A}+\left(p_{A} \circ s_{B}\right) \circ p_{B}=i d_{A} \circ p_{A}+0 \circ p_{B}=p_{A}$

$(I I) p_{B} \circ\left(s_{A} \circ p_{A}+s_{B} \circ p_{B}\right)=\left(p_{B} \circ s_{A}\right) \circ p_{A}+\left(p_{B} \circ s_{B}\right) \circ p_{B}=0 \circ p_{A}+i d_{B} \circ p_{B}=p_{B}$

Observe que usamos o Lema 1.1.8 nas última igualdade de $(I)$ e de $(I I)$, com $0 \circ p_{B}=0$ e $0 \circ p_{A}=0$, respectivamente.

Por $(I)$ e por $(I I)$ concluímos $s_{A} \circ p_{A}+s_{B} \circ p_{B}=i d_{P}$

$(($ iii $) \Longrightarrow($ i) $)$ Dado que vale $($ iii $)$, considere $C$ um objeto de $\mathscr{C}$ e morfismos $f: C \rightarrow A$ e $g: C \rightarrow B$. Defina $h: C \rightarrow P$ por $h=s_{A} \circ f+s_{B} \circ g=i d_{P}$. Queremos ver que $h$ nos dá o diagrama de produto. Vamos compor com $p_{A}$ e $p_{B}$ :

$$
\begin{aligned}
& p_{A} \circ h=p_{A} \circ s_{A} \circ f+p_{A} \circ s_{B} \circ g=f+0=f \\
& p_{B} \circ h=p_{B} \circ s_{A} \circ f+p_{B} \circ s_{B} \circ g=0+g=g .
\end{aligned}
$$

Assim temos que $h$ faz os triângulos comutarem, falta ver que é o único. Suponha $h^{\prime}$ tal que $p_{A} \circ h^{\prime}=f$ e $p_{A} \circ h^{\prime}=g$. Então:

$h^{\prime}=i d_{P} \circ h^{\prime}=\left(s_{A} \circ p_{A}+s_{B} \circ p_{B}\right) \circ h^{\prime}=s_{A} \circ p_{A} \circ h^{\prime}+s_{B} \circ p_{B} \circ h^{\prime}=s_{A} \circ f+s_{B} \circ g=h$

Desta forma temos o diagrama do produto

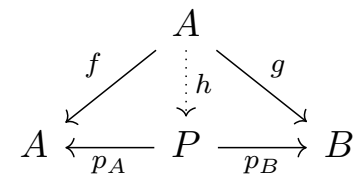


Com isso encerramos a demonstração das equivalências e podemos usá-las para verficiar que $s_{A}=\operatorname{ker}\left(p_{B}\right)$.

Já sabemos que $p_{B} \circ s_{A}=0$. Fixe $X$ objeto de $\mathscr{C}$ e escolha um morfismo $k: X \rightarrow P$ tal que $p_{B} \circ k=0$

Precisamos de uma única fatoração, única ao longo de $k$, tal que $p_{B}$ composta com tal fatoração dê zero. Vamos tentar $p_{A} \circ k: X \rightarrow A$. Temos que $p_{A} \circ s_{A} \circ p_{A} \circ k=i d_{A} \circ p_{A} \circ k=$ $p_{A} \circ k$ e, por outro lado, $p_{B} \circ s_{A} \circ p_{A} \circ k=0 \circ p_{A} \circ k=0=p_{B} \circ k$.

Assim $k=s_{A} \circ p_{A} \circ k$ e a fatoração é única pois $p_{A} \circ s_{A}=i d_{A}, \operatorname{logo}, s_{A}$ é mono.

Então, de fato, o kernel de $p_{B}$ é $s_{A}$.

Analogamente verificamos e $s_{B}=\operatorname{ker}\left(p_{A}\right)$ e as igualdades $p_{A}=\operatorname{coker}\left(s_{B}\right)$ e $p_{B}=$ $\operatorname{coker}\left(s_{A}\right)$ são obtidas dualmente.

Definição 1.1.11 Chamamos de biproduto a quíntupla $\left(P, p_{A}, p_{B}, s_{A}, s_{B}\right)$ com as propriedades descritas em Proposição 2.1.10(iii).

Notação: $P=A \oplus B$.

Definição 1.1.12 Uma categoria aditiva é uma categoria pré-aditiva que possui objeto nulo e biprodutos.

Observe que em uma categoria aditiva o zero de $\operatorname{Hom}(A, B)$ coincide com o morfismo nulo $0_{A B}$.

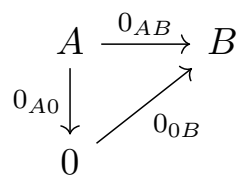

Pois, como argumentamos na demonstração da Proposição 1.1.9, como $i d_{0}$ é zero em $\operatorname{Hom}(0,0)$, fazemos $i d_{0} \circ 0_{A 0}=0_{A 0}$ e, pelo Lema 1.1.8, concluímos que $0_{A 0}$ é zero em $\operatorname{Hom}(A, 0)$. Assim, aplicando novamente o Lema 1.1.8, $0_{A B}=0_{0 B} \circ 0_{A 0}$ é zero de $\operatorname{Hom}(A, B)$.

Como isso encontramos nosso objetivo nessa seção:

Definição 1.1.13 Uma categoria abeliana $\mathscr{C}$ é uma categoria aditiva que satisfaz as seguintes propriedades adicionais:

1. Todo morfismo de $\mathscr{C}$ possui um kernel e um cokernel.

2. Todo monomorfismo de $\mathscr{C}$ é um kernel e todo epimorfismo de $\mathscr{C}$ é um cokernel.

O que fizemos foi gradualmente refinar a noção de categoria pré-aditiva passando por um categoria aditiva e chegamos em categorias abelianas. Poderíamos ter ido direto ao ponto definindo categorias abelianas como uma categoria que possui objeto nulo e todo par de objetos possui produto e coproduto, além das propriedades 1 e 2 enunciadas acima.

Por outro lado, poderíamos ter desenvolvido ainda mais passos de refinamento e, por exemplo, incluir a definição de categoria pré-abeliana entre a aditiva e a abeliana - a saber, é uma categoria aditiva que satisfaz a propriedade 1.

Optamos por essa apresentação acreditando que de tal forma não perdemos as ideias por trás da construção, permitindo o vislumbre de refinamentos da própria noção de categoria abeliana (como categorias trianguladas), sem sermos demasidamente extensos.

É interessante observar que a noção de categoria abeliana é autodual, isto é, a categoria oposta de uma abeliana também é abeliana, isso significa que os resultados apresentados são válidos em suas respectivas versões duais.

Como exemplos, temos novamente Ab, R-Mod e Mod-R e citamos mais dois que aparecem ao longo desse trabalho: a categoria de funtores $\mathscr{C} \rightarrow \mathscr{D}$, onde $\mathscr{D}$ é categoria acabeliana e a categoria dos complexos de cadeias $C h \bullet(\mathscr{C})$ com $\mathscr{C}$ abeliana. 
Dada a importância das categorias abelianas para a Álgebra Homológica, vamos investir certo tempo trabalhando com algumas de suas propriedades.

Proposição 1.1.14 Seja $\mathscr{C}$ um categoria abeliana. Um morfismo $f$ em $\mathscr{C}$ é isomorfismo se, e somente se, $f$ é monomorfismo e epimorfismo.

\section{Demonstração:}

$(\Longrightarrow)$ Como $f$ é é iso, existe $g$ tal que $f g=i d$. Com o morfismo identidade é epi, temos que $f$ é epi. Por outro lado, $f$ ser iso também implica que $g f=i d$ e como a identidade é mono, temos que $f$ é mono.

$(\Longleftarrow)$ Como estamos numa categoria abeliana $f$ mono implica que $f$ é kernel de algum morfismo $g, f=k e r g$. Por definição de kernel e por propriedade do morfismo nulo, $g \circ f=$ $0=0 \circ f$. Como $f$ é epi, $g=0$. Então $f=k e r 0$ e o resultado segue da Proposição 1.1.6.

Antes de prosseguirmos vamos definir rapidamente o que são subobjetos, para qualquer categoria.

Definição 1.1.15 Dizemos que dois monomorfismos $m_{1}: A_{1} \longmapsto A$ e $m_{2}: A_{2} \rightarrow A$ em $A$ são equivalentes se existem morfismos $h: A_{1} \rightarrow A_{2}$ e $h^{\prime}: A_{2} \rightarrow A_{1}$ tais que os seguintes diagramas comutam:
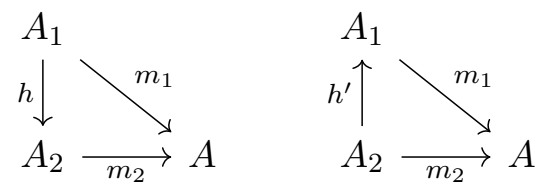

Note que $h$ e $h^{\prime}$ são únicos e isomorfismos inversos.

Uma classe de equivalência de monomorfismos em $A$ é o que chamamos de subobjeto de A.

Estabelecemos uma relação de ordem entre subobjetos de $A$ da seguinte forma:

$$
[m] \leq[n] \text { se, e somente se, existe } f \text { tal que } m=n \circ f
$$

Proposição 1.1.16 Seja $\mathscr{C}$ um categoria abeliana. A intersecção (também visto como o infimo em relação a ordem parcial $\leq$ ) de dois subobjetos sempre existe em $\mathscr{C}$.

\section{Demonstração:}

Considere dois monomorfismos $a: A \longmapsto C$ e $b: B \longmapsto C$ em $\mathscr{C}$. Por definição de categoria abeliana, $a$ e $b$ possuem kernel. Então existem morfismos $f: C \rightarrow D$ e $g: C \rightarrow E$ tais que $a=k e r f$ e $b=k e r g$. Vamos considerar a fatoração $(f, g): C \rightarrow D \times E$, onde $f=p_{D} \circ(f, g)$, com $p_{D}: D \times E \rightarrow D$ a projeção em $D$. Veremos que a interseç̧ão é dada por $\operatorname{ker}(f, g): K \rightarrow C$. Chame $k=k e r(f, g)$, então $f \circ k=p_{D} \circ(f, g) k=p_{D} \circ 0=0 \mathrm{e}$ $g \circ k=0$.

Assim, ainda podemos decompor $k: K \rightarrow C$ em $K \stackrel{a^{\prime}}{\rightarrow} A \stackrel{a}{\rightarrow} C$ e $K \stackrel{b^{\prime}}{\rightarrow} B \stackrel{b}{\rightarrow} C$. Mostraremos agora que $\left(K, a^{\prime}, b^{\prime}\right)$ é pullback de $a$ e $b$. Para isso vamos ter que lidar com uma caça ao diagrama complicada, dependendo do costume. Vamos exibir o diagrama inteiro de uma vez, mas vamos detalhar as passagens.

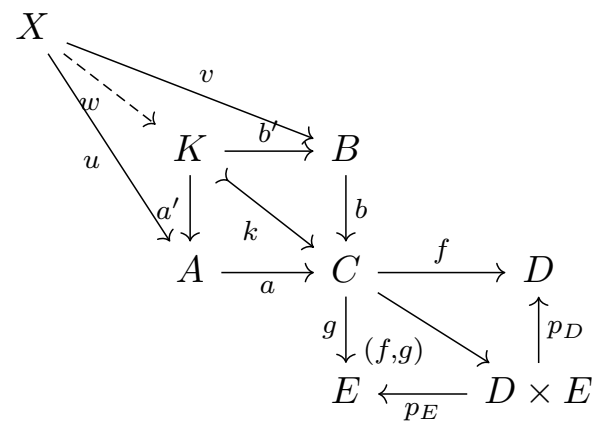


Sabemos que o quadrado superior "interno" comuta, isto é $b b^{\prime}=a a^{\prime}$ e supomos que o quadrado superior "externo" também comuta, isto é, $a u=b v$. Compondo com $f$ dos dois lados: $f a u=f b v$, mas $a=k e r f, \operatorname{logo}, f b v=0 \circ u=0$.

Como também temos $g b v=0$, por definição de kernel, existe um único morfismo $w$ tal que $b v=k w$. Pela decomposição de $k$, temos $k=b b^{\prime}$, assim, $b v=b b^{\prime} w$. Como $b$ é mono, encontramos um único morfismo tal que $v=b^{\prime} w$. Resta ver que $u=a^{\prime} w$.

Usando a outra decomposição $k=a a^{\prime}$ e as igualdades já comentadas: $a u=b v=k w=$ $a a^{\prime} w$. Como $a$ é mono, encontramos o desejado. Então, de fato, $\left(K, a^{\prime}, b^{\prime}\right)$ é pullback de $a$ e $b$ e, portanto, a intersecção dos subobjetos $a$ e $b$.

Note que existe um motivo para verificarmos que justamente o kernel de $(f, g)$ iria nos dar a intersecção. Se estivessemos na categoria dos grupos abelianos $A b$ teríamos

$$
\begin{gathered}
A=\{c \in C \mid f(c)=0\} \text { e } B=\{c \in C \mid g(c)=0\} . \text { Daí: } \\
A \cap B=\{c \in C \mid f(c)=0 \text { e } g(c)=0\}=\{c \in C \mid(f, g)(c)=0\}=\operatorname{ker}(f, g)
\end{gathered}
$$

Uma vez que categorias abelianas podem ser vistas como uma generalização de $A b$, é natural pensar na generalização do que ocorre na categoria $A b$ quando provamos resultados para categorias abelianas.

Proposição 1.1.17 Uma categoria abeliana é finitamente completa e finitamente cocompleta.

\section{Demonstração:}

Vamos mostrar apenas que é finitamente completa pois os argumentos para finitamente cocompleta são análogos.

Um resultado bem conhecido em teoria de categorias é que uma categoria é finitamente completa se, e somente, possui objeto terminal, produtos binários e equalizadores. Uma demonstração disso pode ser encontrada em [Bor94a], Proposição 2.8.2.

Como, por definição de categoria abeliana, já temos que objetos terminais e produtos binários existem, só precisamos verificar a existência dos equalizadores. ${ }^{1}$

Vamos pegar dos morfismos $f, g: A \longrightarrow B$ e procurar um equalizador para eles.

A partir de $f, g$ consideramos os monomorfismos $\left(i d_{A}, f\right): A \rightarrow A \times B$ e $\left(i d_{A}, g\right)$ : $A \rightarrow A \times B$ (são monos porque a composta deles com a projeção em $A$ é identidade, que é um mono - $\left.p_{A} \circ\left(i d_{A}, f\right)=i d_{A}=p_{A} \circ\left(i d_{A}, g\right)\right)$. Se são monos, são subobjetos e, pela Proposição 1.1.16 acima, garantimos que existe uma intersecção entre eles dada pelo diagrama de pullback:

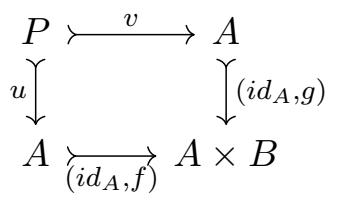

Veremos que $P$ equaliza $f$ e $g$ (assim como na proposição anterior, esse palpite vem do que ocorre no caso dos grupos abelianos).

Estamos pensando em algo da forma

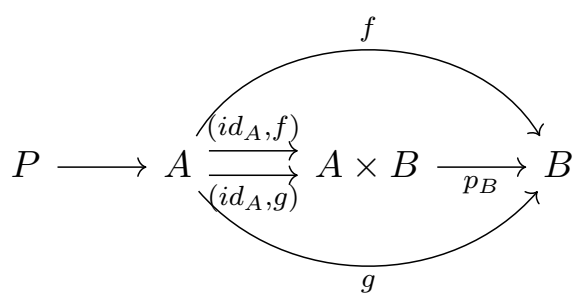

${ }^{1}$ Alternativamente, o equalizador é dado por $e q(f, g)=k e r(f-g)$. 
Usando a projeção em $A$ temos $u=i d_{A} \circ u=p_{A} \circ\left(i d_{A}, f\right) \circ u=p_{A} \circ\left(i d_{A}, g\right) \circ v=i d_{A} \circ v=v$.

Usando a projeção em $B$ temos $f \circ u=p_{B} \circ\left(i d_{A}, f\right) \circ u=p_{B} \circ\left(i d_{A}, g\right) \circ v=g \circ v$.

Com essas duas sequências de igualdade obtemos $f u=g v=g u$. Resta apenas verificar a propriedade universal: Considere $x: X \rightarrow A$ um outro morfismo tal que $f x=g x$, então $\left(i d_{A}, f\right) \circ x=\left(i d_{A}, g\right) \circ x$. Como $P$ é um pullback, existe um único $y: X \rightarrow P$ tal que $u y=v y$. Portanto, $(P, u)$ nos dá um equalizador de $f$ e $g$.

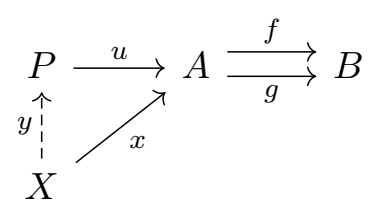

Lema 1.1.18 Sejam $\mathscr{C}$ categoria abeliana e $f: A \Rightarrow B$ um morfismo em $\mathscr{C}$. As seguintes condições são equivalentes:

(i) f é monomorfismo.

(ii) $\operatorname{kerf}=0$.

(iii) Para todo objeto $C$ em $\mathscr{C}$, para todo morfismo $g: C \rightarrow A$, vale que $f \circ g=0$ implica $g=0$.

\section{Demonstração:}

Iremos utilizar apenas que (iii) implica $(i)$ no Teorema a seguir e começamos por essa implicação. Supondo (iii), sejam $h, k$ morfismos em $\mathscr{C}$ tais que $f h=f k$. Então $f h-f k=0$, logo, como vale (iii), $h-k=0$. Portanto, $h=k$. Veja que (i) implica (iii) foi provado na Proposição 1.1.5.

Suponha que vale $(i)$, mostraremos (ii). Queremos o diagrama abaixo é de kernel

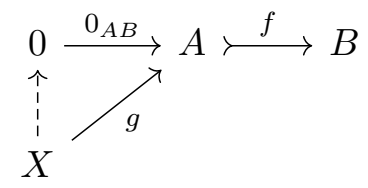

Pela Proposição 1.1.3 temos $O_{0 A} \circ f=0_{K B}$. Seja $g: X \rightarrow A$ um morfirmos tal que $f \circ g=f \circ g=0$. Pela Proposição 1.1.5, $g=0$. Temos que o morfismo $X \rightarrow 0$ é único, pois 0 , sendo objeto nulo é também terminal e por definição de terminal o morfismo que chega em 0 deve ser único. Assim, Kerf $=0$.

Faremos agora $(i i)$ implica $(i)$ para fechar a equivalência.

Sejam $f: A \rightarrow B u: X \rightarrow A$ e $v: X \rightarrow A$ morfismos tais que $f \circ u=f \circ v$. Considere o coequalizador $q: B \rightarrow Q$ de $u$ e $v$. Assim temos o seguinte diagrama de coequalizador:

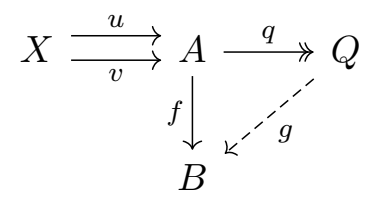

Por definição de categoria abeliana, como $q$ é epi, $q=$ Cokerw para algum morfismo $w$.

Agrupando essas informações encontramos

$$
g \circ w=g \circ q \circ w=g \circ 0=0
$$

Logo, pela propriedade universal do kernel, existe um único $h$ tal que $w=k \circ h$ onde $k$ é o kernel de $f$. Agora o diagrama comutativo passar a ser da forma: 


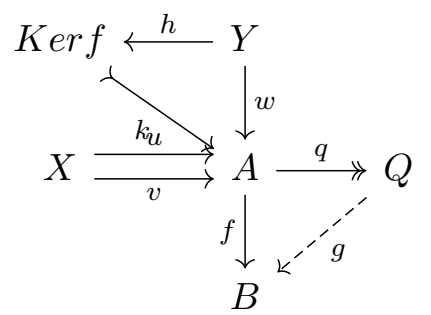

- Como $\operatorname{Kerf}=0$, temos $w=0$ e $q=$ Coker0. Pela Proposição 1.1.6, $q$ é uma identidade, $\operatorname{logo}$, um mono. Como $q \circ u=q \circ v$, então $u=v$ de onde segue que $f$ é mono.

Teorema 1.1.19 Sejam $\mathscr{C}$ categoria abeliana e $f$ um morfismo qualquer em $\mathscr{C}$. Temos que $f$ pode ser fatorado como $f=i \circ p$, onde $i$ é monomorfismo e $p$ é epimorfismo e essa fatoração é única a menos de isomorfismo.

\section{Demonstração:}

Nessa demonstração em vez de denotarmos os objetos pelas letras $A, B, C, \ldots$ vamos denotá-los apenas por "•"pois precisaremos de muitos morfismos e podemos ou não estarmos falando de um mesmo objeto mas assim devemos conseguir diagramas menos poluídos e, mais importante, transmitir que o essencial está nos morfismos e não nos objetos.

Feita essa consideração, tome $f$ morfismo em $\mathscr{C}$ e seja $k=k \operatorname{er} f$ seu kernel. Por definição de kernel temos algo da forma $\underset{l}{i} \stackrel{\substack{i \\:}}{\longrightarrow} \stackrel{f}{\longrightarrow} \bullet$ um único $l$ tal que $h=k \circ l$

Dualmente, chamando $p=$ cokerk (assim já temos $p$ epi) ${ }^{2}$, encontramos o seguinte diagrama:

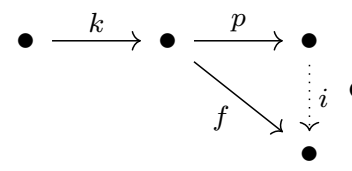
onde $p \circ k=0$ e como $f \circ k=0$, existe um único i tal que $f=i \circ p$.

Se juntarmos os dois diagramas, colocando já na forma que nos será útil em breve:

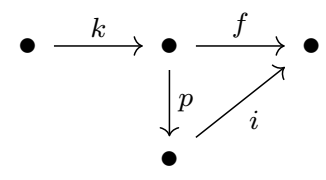

Vamos mostrar que $i$ é monomorfismo.

Pelo Lema 1.1 .18 basta mostrar que $i \circ x=0$ implica $x=0$ para qualquer morfismo $x$. Então suponhamos $i \circ x=0$ e consideremos $q=$ coker $x$ :

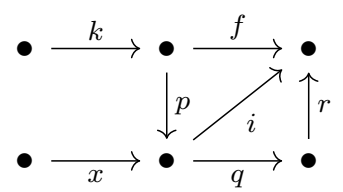

Assim $q \circ x=0$ e como $i \circ x=0$ existe um único $r$ tal que $i=r \circ q$.

Como $p$ e $q$ são cokerneis, ambos são epimorfismos, logo $q \circ p$ também é epi. Por estarmos em categorias abelianas isso implica que $q \circ p$ é o cokernel de algum morfismo. Digamos, $q \circ p=$ cokerh - esse $h$ cumpre todas as funções do $h$ anterior por isso não tem problema algum usar o mesmo.

\footnotetext{
${ }^{2}$ Mais do que isso, é precisamente $p=\operatorname{coker}(k e r f)$, isso será utilizado na seção de complementos sobre categorias abelianas
} 


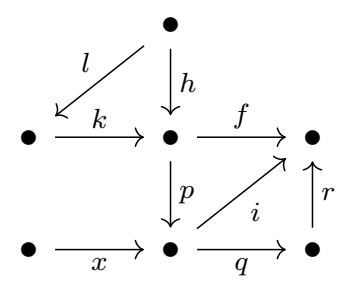

De fato, $f \circ h=i \circ p \circ h=r \circ q \circ p \circ h=r \circ 0=0$.

Por outro lado, $p \circ h=p \circ k \circ l=0 \circ l=0$. Por definição de cokernel, existe uma fatoração única de $p$ ao longo de $q \circ p$, digamos, $p=s \circ q \circ p$. Mas como $p$ é epi, obtemos $s \circ q=i d$.

Sendo $s \circ q$ a identidade, essa composição é portando um mono, logo, $q$ é mono, mas $q \circ x=0$ (não esqueça que $q=\operatorname{coker} x$ ). Portanto, $x=0$.

Temos então $i$ mono e $p$ epi. Agora vamos mostrar que a fatoração é única a menos de isomorfismo.

Seja $f=j \circ q$ uma outra fatoração com $j$ mono e $q$ epi quaisquer.

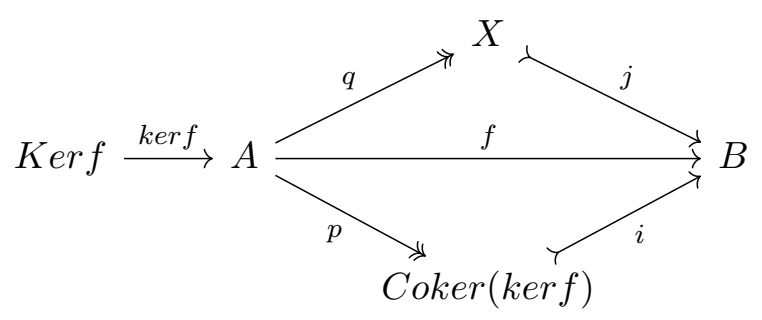

Assim $q \circ \operatorname{ker} f=0$ e, pela propriedade universal do cokernel, existe um único morfismo $u: C o k e r(k e r f) \rightarrow X$ tal que $u \circ p=q$. Como $q$ é epi, $u$ também é epi.

Veja ainda que $i \circ p=f=j \circ q=j \circ u \circ p$. Como $p$ é epi, $i=j \circ u$ de onde segue que $u$ é mono porque $i$ é mono.

Verificamos que $u$ é epi e mono, logo, pela Propopsição 1.1.14, é um iso. Pelo que vimos, é o único iso que fazer o diagrama abaixo comutar.

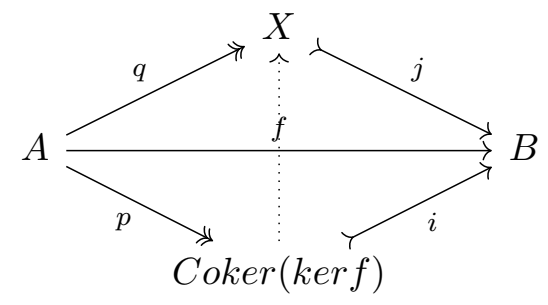

Portanto, as fatorações $j \circ q$ e $i \circ p$ são isomorfas.

Daqui em diante a notação fica mais carregada, pois iremos manter o ker e coker para os morfismos e objetos com a única distinção que no primeiro caso utilizaremos letras minúscula e no segundo iniciaremos com letra maiúscula, como fizemos para mostrar que a fatoração em epi e mono é única a menos de isomorfismo.

A propriedade 2 da definição 1.1.13 é enunciada de forma diferente no artigo de A. Grothendieck "Para todo morfismo $f$, temos que Coimf $\cong \operatorname{Imf}$ é um isomorfismo". Apesar de ser bem conhecido que os dois enunciados são equivalentes, não encontramos uma demonstração e aproveitaremos esse espaço para fazer isso de forma detalhada.

Algo que não mencionamos mas nos será útil é que todo mono é kernel de seu cokernel (dualmente, todo epi é cokernel de seu kernel).

Lema 1.1.20 Todo monomorfismo é kernel de seu cokernel.

\section{Demonstração:}

De fato, seja $f: A \rightarrow B$ um mono, pela propriedade 2 da definição de categoria abeliana, $f=k e r g$ para algum $g: B \rightarrow C$. Como $g \circ f=g \circ k e r g=0$, temos o seguinte diagrama de cokernel: 


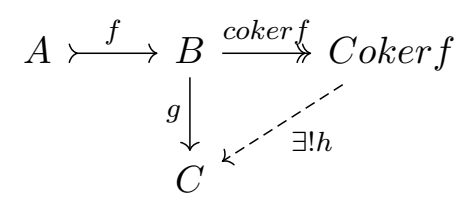

Seja $p: D \rightarrow B$ tal que $($ coker $f) \circ p=0$. Como $f=$ kerg e $g \circ p=h \circ($ coker $f) \circ p=0$, existe um único $q: D \rightarrow A$ tal que $p=f \circ q$, ou seja, $f=k e r(\operatorname{coker} f)$, o mono $f$ é justamente quem faz o papel de kernel do morfismo coker $f$, como queríamos.

Por um instante esqueceremos o axioma 2 de categoria abeliana. Note que o primeiro axioma nos permite a seguinte construção:

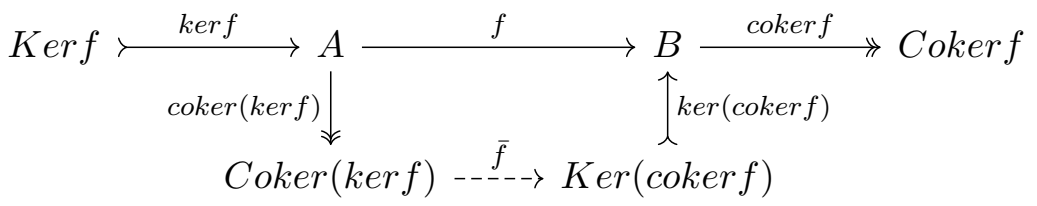

onde $f$ é um morfismo qualquer e Coker $(\operatorname{ker} f)=\operatorname{Coimf}$ e $\operatorname{Ker}(\operatorname{coker} f)=\operatorname{Im} f$. Primeiro veremos que existe um único morfismo $\bar{f}: \operatorname{Coker}(\operatorname{ker} f) \rightarrow \operatorname{Ker}(\operatorname{coker} f)$

Por definição de cokernel, $(\operatorname{coker}(\operatorname{ker} f)) \circ k e r f=0$ e, por definição de kernel, $f \circ k e r f=$ 0 . Pela propriedade universal do cokernel existe uma única $u: \operatorname{Coker}(k e r f) \rightarrow B$ tal que $f=u \circ \operatorname{coker}(\operatorname{ker} f) \quad(\star)$.

Agora temos $(\operatorname{coker} f) \circ \operatorname{ker}(\operatorname{coker} f)=0$ (por definição de kernel) e:

$$
\begin{aligned}
(\operatorname{coker} f) \circ u \circ \operatorname{coker}(\operatorname{ker} f) & =\operatorname{coker} f \circ f & & \text { por }(\star) \\
& =0 & & \text { por definição de cokernel } \\
& =0 \circ \operatorname{coker}(\operatorname{ker} f) & &
\end{aligned}
$$

Como coker $(k e r f)$ é epi, temos $(\operatorname{coker} f) \circ u=0$. Então, pela propriedade universal do kernel existe uma única $\bar{f}: \operatorname{Coker}(\operatorname{ker} f) \rightarrow \operatorname{Ker}(\operatorname{coker} f)$ tal que $u=(\operatorname{ker}(\operatorname{coker} f)) \circ \bar{f}$. Portanto, $f=k e r(\operatorname{coker} f) \circ f$ o coker $(k e r f)$ e como ker(cokerf) é mono e coker $(k e r f)$ é epi, $\bar{f}$ é o único morfismo que faz o diagrama comutar.

Uma vez que $\bar{f}$ existe, queremos:

Proposição 1.1.21 Seja $\mathscr{C}$ uma categoria qua satisfaz AB1, isto é, todo morfismo possui kernel e cokernel. Então são equivalentes:

2 Todo mono é kernel e todo epi é cokernel.

2' O morfismo $\bar{f}:$ Coker $(\operatorname{ker} f) \rightarrow \operatorname{Ker}($ cokerf $)$ é iso.

\section{Demonstração:}

Vamos começar pelo lado mais fácil.

$\left(2^{\prime} \Longrightarrow 2\right)$ Entendo $f: A \rightarrow B$ e $\operatorname{Ker}(\operatorname{coker}(f))$ como objetos sobre $B$, vamos mostrar que $f \cong k e r(\operatorname{coker}(f))$ quando $f$ é mono.

Já vimos que $f=k \operatorname{ker}(\operatorname{coker} f) \circ \bar{f} \circ(\operatorname{coker}(\operatorname{ker} f))$, logo, $f \leq \operatorname{ker}(\operatorname{coker}(f))$.

Como $f$ é mono, temos quer coker $(k e r(f))$ é mono. Logo, como já era epi por ser um coequalizador, coker $(k e r(f))$ é isomorfismo. Usando $2^{\prime}$, a composição $\bar{f} \circ(\operatorname{coker}(k e r f))$ é um iso, então adminte inversa $h: A \rightarrow \operatorname{Ker}(\operatorname{coker}(f))$ tal que $f \circ h=\operatorname{ker}(\operatorname{coker}(f))$, isto é, $\operatorname{ker}(\operatorname{coker}(f)) \leq f$.

Portanto $f \cong k e r(\operatorname{coker}(f))$.

Analogamente, se $f$ for epi concluímos que $f$ cokernel de seu kernel.

$\left(2 \Longrightarrow 2^{\prime}\right)$ Como estamos assumindo $A B 2$, podemos usar os teoremas vistos até agora. $\mathrm{Na}$ demonstração do Teorema 1.1.17 vimos não apenas que um morfismo $f$ se decompõe em epi e mono, mas que o epi é justamente da forma coker $(k e r f)$. Veremos agora que o $i=\operatorname{ker}(\operatorname{cokerf})$. 
Como $p$ é epi, temos

$$
g \circ f=0 \Longleftrightarrow g \circ i \circ p=0 \Longleftrightarrow g \circ i \circ p=0 \circ p \Longleftrightarrow g \circ i=0
$$

Note que isso nos dá que Coker $f \cong$ Cokeri. Com efeito, sabemos que (coker $f) \circ f=0$, $\operatorname{logo},($ coker $f) \circ i=0$. Pela propriedade universal do cokernel existe um único morfismo Cokeri $\rightarrow$ Cokerf. Analogamente, (cokeri) $\circ i=0$ implica (cokeri) $\circ f=0$ e, portanto, existe um único morfismo Coker $f \rightarrow$ Cokeri. A existência desses dois morfismos únicos nos garante o isomorfismo. Como, pela Proposição 1.1.20, todo mono é kernel de seu cokernel $i=k e r($ cokeri). Pelo iso acima, $i \cong k e r(\operatorname{cokerf})$.

Assim, por um lado temos $f=k e r(\operatorname{cokeri}) \circ \operatorname{coker}(\operatorname{kerp}) \cong \operatorname{ker}(\operatorname{coker} f) \circ \operatorname{coker}(\operatorname{ker} f)$, isto é, existem isomorfismos $g$ e $h$ tais que $k e r($ cokeri $)=k e r($ coker $f) \circ g$ e coker $($ kerp $)=$ $h \circ \operatorname{coker}(\operatorname{kerf})$.

Por outro lado temos $f=\operatorname{ker}(\operatorname{coker} f) \circ \bar{f} \circ \operatorname{coker}(k e r f)$, então:

$$
\operatorname{ker}(\operatorname{coker} f) \circ g \circ h \circ \operatorname{coker}(\operatorname{ker} f)=\operatorname{ker}(\operatorname{coker} f) \circ \bar{f} \circ \operatorname{coker}(\operatorname{ker} f)
$$

Usando que ker (coker $f)$ é mono, obtemos $g \circ h \circ \operatorname{coker}(k e r f)=\bar{f} \circ \operatorname{coker}(k e r f)$. Como coker (kerf) é epi, isso implica que $\bar{f}=g \circ h$ e, portanto, $\bar{f}$ é um iso.

Definição 1.1.22 Temos a seguinte nomenclatura para imagem e coimagem de um morfismo $f$, respectivamente, $\operatorname{Im}(f)=\operatorname{Ker}(\operatorname{Coker}(f))$ e $\operatorname{Coim}(f)=\operatorname{Coker}(\operatorname{Ker}(f))$.

Observe que se tivermos $A \stackrel{f}{\rightarrow} B \stackrel{g}{\rightarrow} C$ e pensarmos em $\operatorname{Im} f$ e $\operatorname{Ker}(g)$ como subobjetos de $B$, então $g \circ f=0$ se, e somente se, $\operatorname{Im} f \leq \operatorname{Ker}(g)$.

Definição 1.1.23 Seja $\mathscr{C}$ categoria abeliana. Dizemos que uma sequência $A \stackrel{f}{\rightarrow} B \stackrel{g}{\rightarrow} C$ em $\mathscr{C}$ é exata se a imagem de $f$ e o kernel de $g$ coincidem. Em outras palavras, se temos a seguinte fatoração

$$
A \stackrel{p}{\longrightarrow} \operatorname{Kerg} \stackrel{\text { kerg }}{\longrightarrow} B
$$

$\operatorname{Com} p=\operatorname{ker}(\operatorname{coker} f)$.

Considerando $\operatorname{Im}(f)$ e $\operatorname{Ker}(g)$ como subobjetos de $B$, observe que $g \circ f=0$ se, e somente se, $\operatorname{Im}(f) \leq \operatorname{Ker}(g)$.

Proposição 1.1.24 A sequência $A \stackrel{f}{\rightarrow} B \stackrel{g}{\rightarrow} C$ em $\mathscr{C}$ é exata se, e somente se, $g$ admite fatoração em epi e mono da forma $B \stackrel{\text { cokerf }}{\longrightarrow}$ Cokerf $\stackrel{m}{\longrightarrow} C$

\section{Demonstração:}

$(\Longrightarrow)$ Dada a sequência exata, temos o seguinte diagrama comutativo:

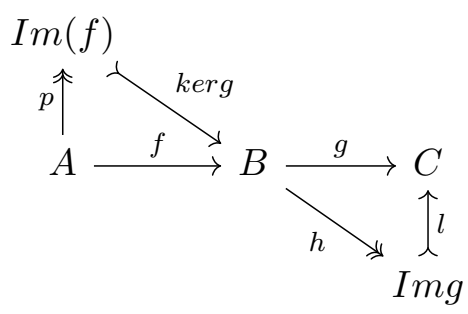

Uma vez que $g \circ f=0$, temos $l \circ h \circ f=0$. Como $l$ é mono, pela Proposição 1.1.5, $h \circ f=0$. Logo, existe uma única $\alpha:$ Coker $f \rightarrow \operatorname{Img}$ tal que $\alpha$ o coker $f=h$. Além disso, $h$ é epi, assim, $\alpha$ também é.

Por outro lado, Img $=$ Coker $($ Kerg $)$. Logo:

$$
(\operatorname{cokerf}) \circ(\operatorname{kerg})=(\operatorname{coker} f) \circ(\operatorname{coker}(\operatorname{ker} f))=0
$$


. Então existe uma única $\beta:$ Img $\rightarrow$ Cokerf tal que $\beta \circ h=\operatorname{cokerf.~Mas~} h$ e cokerf são epis e $\beta \circ \alpha \circ$ cokerf $=\operatorname{coker} f$, então $\beta \circ \alpha=i d$, de onde segue que $\alpha$ é mono. Temos $\alpha$ mono e epi, pela Proposição 1.1.14, $\alpha$ é iso. Agora chame $m=l \circ \alpha$, como $\alpha$ é iso e $l$ é mono, $m$ é mono e encontramos a fatoração desejada.

$(\Longleftarrow)$ Como $m$ é mono, $\operatorname{Kerg}=\operatorname{Ker}(m \circ \operatorname{coker} f)=\operatorname{Ker}(\operatorname{coker} f)=\operatorname{Imf}$

A sequência longa (e finita) $\ldots \rightarrow A_{n} \rightarrow A_{n+1} \rightarrow \ldots$ é exata se cada dois morfismos consecutivos são exatos.

Demais definições (sequência exata curta, por exemplo) e os principais resultados que seguem da noção de sequências exatas na categoria de módulos sobre um anel valem de forma análoga em categorias abelianas. Recomendamos [Bor94b] para quem desejar ver os enunciados e respectivas demonstrações explicitamente para o caso das categorias abelianas.

Poderiamos agora definir pseudoelementos, mas faremos isso apenas na seção 7. Alguns cometários que fogem do contexto da Álgebra Homológica merecem ser feitos e preferimos deixá-los para o fim do capítulo.

Contudo, o Lema de Serpente será necessário antes então iremos enunciá-lo aqui e demonstrá-lo mais tarde.

Lema 1.1.25 (Lema da Serpente) Considere o seguinte diagrama em uma categoria abeliana

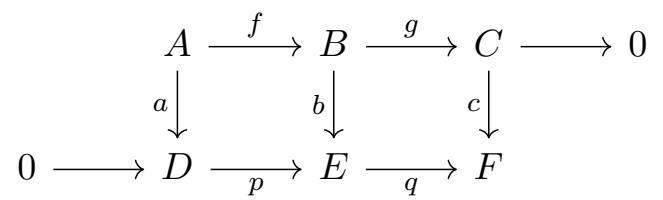

onde $A \rightarrow B \rightarrow C \rightarrow 0$ e $0 \rightarrow D \rightarrow E \rightarrow F$ são exatas e os quadrados comutam. Então o diagrama abaixo comuta

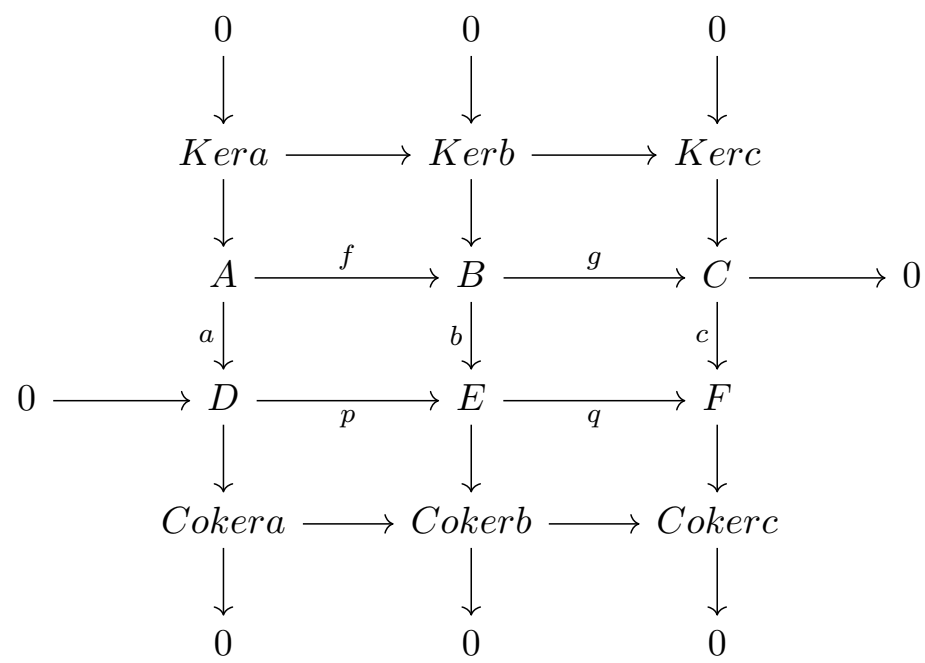

e existe morfismo d : Kerc $\rightarrow$ Cokera tal que a sequência $0 \rightarrow$ Kera $\rightarrow$ Kerb $\rightarrow$ Kerc $\stackrel{\partial}{\rightarrow}$ Cokera $\rightarrow$ Cokerb $\rightarrow$ Cokerc $\rightarrow 0$ é exata

\section{$1.2 \quad$ Funtores Exatos}

Essa seção é para apenas definir funtores exatos e mostrar alguns exemplos que serão necessários mais adiante. A ideia é que um funtor exato preserve a exatidão de sequências exatas.

Definição 1.2.1 Dadas duas categorias abelianas $\mathscr{C}$ e $\mathscr{C}^{\prime}$, um funtor $F: \mathscr{C} \rightarrow \mathscr{C}^{\prime}$ é dito aditivo quando o mapa

$$
F_{A, B}: \operatorname{Hom}_{\mathscr{C}}(A, B) \rightarrow \operatorname{Hom}_{\mathscr{C}^{\prime}}(F(A), F(B)), f \mapsto F(f)
$$


é um homomorfismo de grupo para todos os objetos $A$ e $B$ de $\mathscr{C}$.

Definição 1.2.2 Sejam $\mathscr{C}$ e $\mathscr{C}^{\prime}$ categorias abelianas e um funtor aditivo $F: \mathscr{C} \rightarrow \mathscr{C}^{\prime}$. Considere uma sequência exata $0 \rightarrow A \rightarrow B \rightarrow C \rightarrow 0$. Dizemos que $F$ é:

1. Exato se $0 \rightarrow F(A) \rightarrow F(B) \rightarrow F(C) \rightarrow 0$ é exata.

2. Exato à esquerda se $0 \rightarrow F(A) \rightarrow F(B) \rightarrow F(C)$ é exata.

3. Exato à direita se $F(A) \rightarrow F(B) \rightarrow F(C) \rightarrow 0$ é exata.

Proposição 1.2.3 Seja $\mathscr{C}$ categoria abeliana. Então $\operatorname{Hom}_{\mathscr{C}}(-, I)$ é funtor (contravariante) exato à esquerda para todo $I \in \mathscr{C}$.

\section{Demonstração:}

Seja $0 \rightarrow A \stackrel{f}{\rightarrow} B \stackrel{g}{\rightarrow} C \rightarrow 0$ sequência exata. Vamos fazer $g_{\#}=H_{\mathscr{C}}(g, I)$ e $f_{\#}=\operatorname{Hom}_{\mathscr{C}}(f, I)$

Seja $h \in H_{\mathscr{C}}(C, I)$ tal que $g_{\#}(h)=h \circ g=0$, então $h=0$, pois $g$ é epi. Logo, $g_{\#}$ é mono.

Temos também que $f_{\#} \circ g_{\#}(h)=f_{\#}(h \circ g)=h \circ g \circ f=h \circ 0=0$. Então $I m g_{\#} \leq k e r f_{\#}$

Por fim, veremos que $k e r f_{\#} \leq I m g_{\#}$. Tome $k \in \operatorname{Hom}_{\mathscr{C}}(B, I)$ tal que $f_{\#}(k)=k \circ f=0$, isto é, $k \in \operatorname{ker} f_{\#}$.

Proposição 1.1.24, $g$ se fatora pelo Coker $f$. Por propriedade do cokernel, dado $k: B \rightarrow$ $I$ onde $k \circ f=0$, existe um único morfismo $t:$ Coker $f \rightarrow I$ tal que $k=t \circ f=0$. Fatoramos de $t$ de forma que existe $h \in H_{\mathscr{C}}(C, I)$ tal que o seguinte diagrama comuta

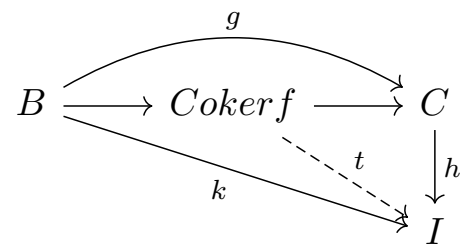

Então $k=t \circ \operatorname{coker} f=h \circ m \circ \operatorname{coker} f=h \circ g=g_{\#}(h), \operatorname{logo}, k \in I m g_{\#}$ e assim verificamos que $I m g_{\#}$ coincide com $k e r f_{\#}$.

Para as próximas duas proposições vamos definir uma categoria $\mathscr{C}^{+}$onde um objeto $A$ de $\mathscr{C}^{+}$consiste de uma família de morfismos $f_{i}^{A}: A_{i} \rightarrow A_{i-1}$ de $\mathscr{C}$ e morfismos $h: A \rightarrow B$ em $\mathscr{C}^{+}$são morfismos $h_{i}: A_{i} \rightarrow B_{i}$ em $\mathscr{C}$, com $i \in \mathbb{Z}$ de forma que o seguinte diagrama comuta

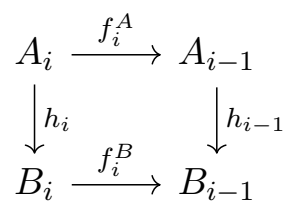

Assim, se $\mathscr{C}$ é uma categoria abeliana então $\mathscr{C}^{+}$também é.

Nessas condições, quando dizemos que $0 \rightarrow A \rightarrow B \rightarrow C \rightarrow 0$ é exata em $\mathscr{C}^{+}$, isso significa que $0 \rightarrow A_{i} \rightarrow B_{i} \rightarrow C_{i} \rightarrow 0$ é exata para todo $i \in \mathbb{Z}$.

Proposição 1.2.4 O funtor $\operatorname{Ker} f_{i}(-): \mathscr{C}^{+} \rightarrow \mathscr{C}$ é exato à esquerda para todo $i \in \mathbb{Z} . A$ notação usada é $\operatorname{Kerf}_{i}(A)=\operatorname{Kerf} f_{i}^{A}$.

\section{Demonstração:}

Vamos começar estudando o seguinte diagrama: 


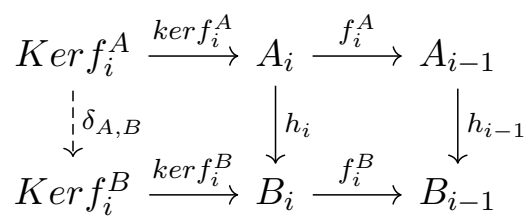

Por construção temos que o quadrado da direita comuta e a composição $h_{i} \circ \operatorname{ker} f_{i}^{A}$ é um morfismo que vai de $\operatorname{Kerf} f_{i}^{A}$ em $B_{i}$. Além disso, pela comutatividade do diagrama e propriedade do kernel de $f_{i}^{A}$

$$
f_{i}^{B} \circ h_{i} \circ \operatorname{kerf}_{i}^{A}=h_{i-1} \circ f_{i}^{A} \circ \operatorname{ker} f_{i}^{A}=0
$$

Então, pela propriedade universal do kernel de $f_{i}^{B}$, existe um único morfismo $\delta_{A, B}$ que faz o diagrama da esquerda comutar.

Procedemos analogamente para encontrar $\delta_{B, C}: \operatorname{Kerf} f_{i}^{B} \rightarrow \operatorname{Kerf} f_{i}^{C}$

Agora observe o seguinte diagrama:

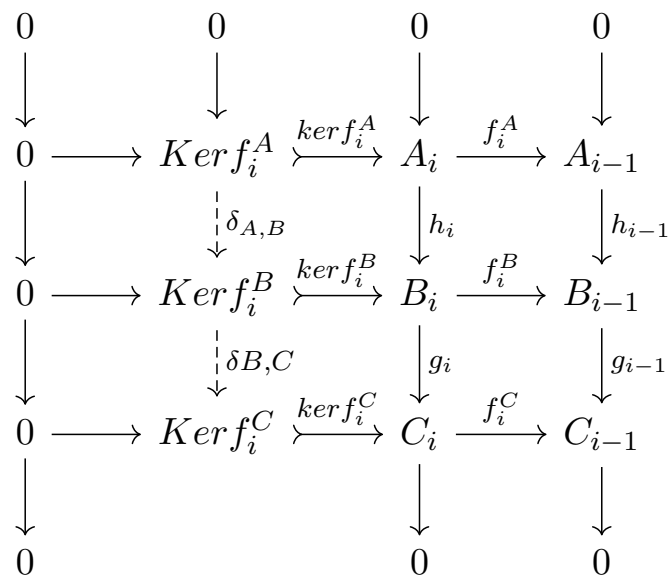

Como $h_{i} \circ \operatorname{ker} f_{i}^{A}=\operatorname{kerf}_{i}^{B} \circ \delta_{A, B}$, kerneis são monos e $h_{i}$ é mono, temos que $\delta_{A, B}$ é momo. Além disso, $g_{i} \circ h_{i}=0$ implica que $\delta_{B, C} \circ \delta_{A, B}=0$, logo, $\delta_{A, B} \leq \delta_{B, C}$. Resta então verificar que $\operatorname{ker} \delta_{B, C} \leq \operatorname{Im} \delta_{A, B}$.

Para facilitar faremos a caça como se estivéssemos em $R$-módulos (idealmente usariamos os pseudoelementos introduzidos na seção 1.7). Seja $k^{\prime} \in k e r \delta_{B, C}$, vamos verificar que $k^{\prime}=\delta_{B, C}(b)$ para algum $b \in \operatorname{Kerf} f_{i}^{A}$.

Tome $k^{\prime} \in \operatorname{Ker}_{B, C}$, então $\operatorname{kerf}_{i}^{B}\left(k^{\prime}\right) \in B_{i}$ e fazemos $g_{i} \circ \operatorname{kerf} f_{i}^{B}\left(k^{\prime}\right)=\operatorname{ker}_{i}^{C} \circ$ $\delta_{B, C}\left(k^{\prime}\right)=0$, pois os quadrados comutam e $k^{\prime} \in \operatorname{ker} \delta_{B, C}$.

Essa igualdade nos dá que $\operatorname{kerf}_{i}^{B}\left(k^{\prime}\right) \in \operatorname{Kerg}_{i}$. Como a sequência $0 \rightarrow A_{1} \stackrel{h_{i}}{\longrightarrow} B_{i} \stackrel{g_{i}}{\rightarrow}$ $C_{i} \rightarrow 0$ é exata, temos $\operatorname{kerg}_{i} \leq \operatorname{Im} h_{i}$, de onde segue que $\operatorname{kerf}_{i}^{B}\left(k^{\prime}\right)=h_{i}(a)$ para algum $a \in A_{i}$.

Mas note que $k^{\prime} \in \operatorname{Ker} \delta_{B, C}$ implica $k^{\prime} \in \operatorname{Ker} f_{i}^{B}, \operatorname{logo}, h_{i}(a)=\operatorname{ker} f_{i}^{B}\left(k^{\prime}\right)=0$

Agora seja $b \in \operatorname{Ker} f_{i}^{A}$, então $h_{i} \circ \operatorname{ker} f_{i}^{A}(b)=0$ e obtemos $h_{i}(a)=0=h_{i} \circ \operatorname{ker} f_{i}^{A}(b)$. Como $h_{i}$ é mono, $a=k e r f_{i}^{A}(b)$.

Assim, $\operatorname{kerf}_{i}^{B}\left(k^{\prime}\right)=h_{i}(a)=h_{i} \circ \operatorname{ker}\left(f_{i}^{A}(b)\right)=\operatorname{kerf}_{i}^{B} \circ \delta_{A, B}(b)$. Como $\operatorname{ker} f_{i}^{B}$ é mono, $k^{\prime}=\delta_{A, B}(b)$, como queríamos.

Proposição 1.2.5 O funtor Coker $f_{i}(-): \mathscr{C}^{+} \rightarrow \mathscr{C}$ é exato à direita para todo $i \in \mathbb{Z}$. A notação usada é Coker $f_{i}(A)=\operatorname{Coker}_{i}^{A}$.

\section{Demonstração:}

A demonstração desse resultado é dual ao anterior. 


\subsection{Homologia e Cohomologia}

Podemos dizer que (co)homologia associa sequências de objetos algébricos com outros objetos matemáticos. Em Topologia Algébrica, por exemplo, esse outro objeto matemático é um espaço topológico e, nesse caso, temos uma interpretação geométrica de forma que a homologia é capaz de calcular o "número de buracos" da estrutura, além do quão não exata é a sequência associada. Nessa seção nos guiamos na aprensetação feita em [GM02] e [Wei95]

Vamos dar alguma motivação e tornar preciso os comentários feitos acima.

Definição 1.3.1 Seja $\mathscr{C}$ uma categoria abeliana. Um complexo de cadeias de $\mathscr{C}$ é uma sequência $\left\{C_{i}\right\}$ de objetos de $\mathscr{C}$ equipada com morfismos, chamados operadores de bordo, $d_{i}^{C}: C_{i} \rightarrow C_{i-1}$ tais que $d_{i-1}^{C} \circ d_{i}^{C}=0$, com $i \in \mathbb{Z}$. Denotamos esse complexo por C.

Invertendo a direção das flechas e tomando o cuidado necessário com os índices, definimos a noção de complexo de cocadeia e mudamos a notação para $C$.

Sempre que não houver confusão, denotaremos os operadores de bordo apenas por $d$.

Definição 1.3.2 Sejam $C$ • e $D$ • complexos de cadeias. Definimos um morfismo entre complexos $h_{\bullet}: C_{\bullet} \rightarrow D_{\bullet}$ uma coleção de morfismos $h_{i}: C_{i} \rightarrow D_{i}$ tal que o seguinte diagrama comuta:

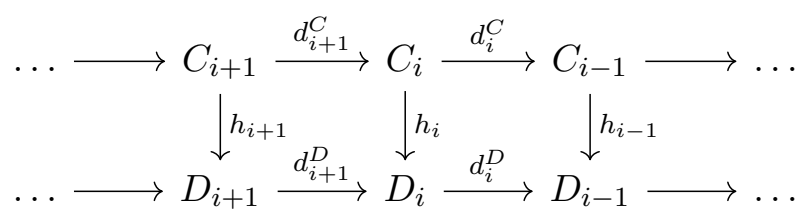

Chamamos de $C h(\mathscr{C})$ a categoria dos complexos de cadeias.

Observe que $C h(\mathscr{C})$ é subcategoria plena de $\mathscr{C}^{+}$.

Observação 1.3.3 A categoria $C h(\mathscr{C})$ é abeliana. É possível encontrar uma demonstração para isso em [Wei95] (Teorema 1.2.3).

Note que o índice $i$ não precisa parar em nenhum momento e pode assumir valores negativos. Contudo, normalmente queremos que a sequência pare em algum dos lados (qual lado depende se é cadeia ou cocadeia) e para isso fazemos $C_{i}=0$ para todo $i \leq 0$.

A propriedade $d^{2}=0$, nos garante que $0 \subseteq I m d_{i+1} \subseteq \operatorname{Kerd}_{i} \subseteq C_{n}$, isso é fundamental para que possamos definir homologia:

Definição 1.3.4 Seja $C_{\bullet}$ complexo de cadeia. Definimos o $q$-ésimo objeto de homologia de $C \bullet$ por

$$
H_{q}\left(C_{\bullet}\right)=\operatorname{Ker}\left(d_{q}\right) / \operatorname{Im}\left(d_{q+1}\right)
$$

Em mais detalhes, para categorias abelianas, se temos um monomorfismo $f: A \longmapsto B$ o quociente $B / A$ denota o cokernel de $f$. Dessa forma, $H_{q}\left(C_{\bullet}\right)=\operatorname{coker}\left(\operatorname{Im}\left(d_{q+1}\right) \longmapsto\right.$ $\operatorname{Ker}\left(d_{q}\right)$ ), onde $\operatorname{Im} d_{q+1} \subseteq \operatorname{Kerd}_{q}$ nos permite formar o seguinte diagrama comutativo

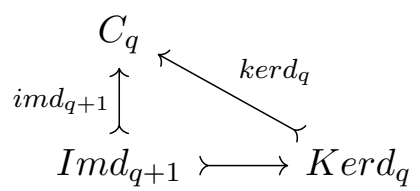

Para o $q$-ésimo objeto de cohomologia fazemos

$$
H^{q}\left(C^{\bullet}\right)=\operatorname{Ker}\left(d_{q}\right) / \operatorname{Im}\left(d_{q+1}\right)=\operatorname{coker}\left(\operatorname{Im}\left(d_{q+1}\right) \longmapsto \operatorname{Ker}\left(d_{q}\right)\right)
$$


Note que se a sequência dos complexos for exata então é claro que $H^{q}=0$ para todo $q$. Por isso dizemos que a homologia é uma forma de calcular quão não exata é a sequência.

Dissemos no início da seção que a homologia poderia associar sequências de objetos algébricos com espaços topológicos. Vamos dar um exemplo de como isso funciona tomando o círculo $S^{1}$.

O que precisamos é dizer como são são os operadores de bordo, para isso precisamos decidir uma boa forma de enxergar o espaço $S^{1}$. Optamos por usar simplexos, isto é, calcularemos a homologia simplicial.

Definição 1.3.5 Denominamos por simplexo n-dimensional o espaço topológico

$$
\Delta_{n}=\left\{\left(x_{0}, \ldots, x_{n}\right) \in \mathbb{R}^{n+1} \mid \sum_{i=0}^{n} x_{i}=1, x_{i} \geq 0\right\},
$$

onde, para cada $x_{i}=1$, o ponto $e_{i}=(0, \ldots 0,1,0, \ldots, 0)$, com 1 na $i$-ésima posição, é o $i$-ésimo vértice de $\Delta_{n}$ e o conjunto dos vértices é ordenado por $e_{0}<\ldots<e_{n}$.

Basicamente, simplexos são grupos abelianos livres formados por pontos, segmentos de retas, triângulos, tetraedros e suas demais contrapartes n-dimensionais.

Ainda, para cada $n$-simplexo existe uma $(n-1)$-face que é um mapa $\delta_{k}: \Delta_{n} \rightarrow \Delta_{n-1}$ que retira a $k$-entrada, isto é, $\delta_{k}\left(x_{0}, \ldots, x_{n}\right)=\left(x_{0}, \ldots, \widehat{i_{k}}, \ldots, x_{n}\right)$.

Com isso, o operador de bordo é definido como a soma alternada dessas faces, dado $\alpha \in C_{i}, d_{i}(\alpha)=\sum_{k=0}^{1} \delta_{k}(x)$, onde $x=\left(x_{0}, \ldots, x_{n}\right)$. Isso é o suficiente para prosseguirmos com o exemplo.

Temos que $S^{1}$ é um 2-simplexo como na figura abaixo, onde $u<v<w$.

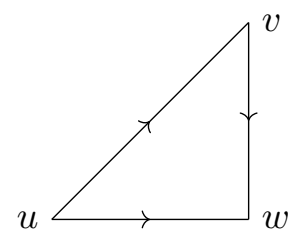

onde $u, v, w$ são 0 -simplexos com $u$ o menor de todos pois duas flechas saem dele e $w$ o maior pois duas flechas chegam (ou, como vimos na definição de simplexo, $u=e_{1}, v=$ $\left.e_{2}, w=e_{3}\right)$. Os 1-simplexos são os vértices $u v, v w$ e $u w$.

Temos uma sequência do tipo $\ldots \stackrel{d_{3}}{\longrightarrow} C_{2} \stackrel{d_{2}}{\longrightarrow} C_{1} \stackrel{d_{1}}{\longrightarrow} C_{0} \stackrel{d_{0}}{\longrightarrow} 0$.

Com $C_{i}$ é gerado pelos $i$-simplexos, logo a sequência acima se reduz à

$$
0 \stackrel{d_{2}}{\longrightarrow}<u v, v w, u w>\stackrel{d_{1}}{\longrightarrow}<u, v, w>\stackrel{d_{0}}{\longrightarrow} 0
$$

Observe que $C_{2}=0$ pois $S^{1}$ é formado apenas pelos pontos e segmentos de reta, disso já obtemos $\operatorname{Im} d_{2}=0$.

Agora fazemos $d_{1}(u v)=v-u, d_{1}(v w)=w-v$ e $d_{1}(u w)=w-u$. Desta forma, $I m d_{1}=<v-u, w-v, w-u>$. Dados $a, b, c$ inteiros, temos

$$
d_{1}(a u v+b v w+c u w)=(-a-b) u+(a-b) v+(b+c) w=0 \Longleftrightarrow a=b=-c
$$

Isso significa que $k e r d_{1}$ possui um único gerador, em outras palavras, $K e r d_{1}=\mathbb{Z}$. Além disso, é claro que $I m d_{0}=0$ e $\operatorname{Kerd}_{0}=\langle u, v, w\rangle$. Portanto, $H_{1}\left(S^{1}\right)=\mathbb{Z}$ e $H_{0}\left(S^{1}\right)=\mathbb{Z}$.

Por questões teóricas que não entraremos em detalhes a homologia do grau 0 sempre é $\mathbb{Z}$, mas o resultado algébrico $H_{1}\left(S^{1}\right)=\mathbb{Z}$ é interpretado geometricamente como dizer que $S^{1}$ possui um único buraco de dimensão 1 . 
Calculamos a homologia simplicial, mas poderíamos ter usado outras (a singular, por exemplo) e obteríamos o mesmo resultado. Isso ocorre porque essas e muitas outras satisfazem o que chamam de axiomas de Eilenberg-Steenrod, um livro que fala um pouco disso é [Bre13].

Agora vamos voltar para como os complexos de cadeia e as homologias se relacionam de um ponto de vista geral.

Proposição 1.3.6 Um morfismo entre complexos $h_{\bullet}: C_{\bullet} \rightarrow D_{\bullet}$ induz um morfismo $H(h): H_{q}\left(C_{\bullet}\right) \rightarrow H_{q}\left(D_{\bullet}\right)$. Desta forma, $H_{q}(-): C h(\mathscr{C}) \rightarrow \mathscr{C}$ determina um funtor.

\section{Demonstração:}

Sabemos por 1.2.4 que $\operatorname{Kerd}_{q}^{C}$ é um funtor, então existe $f_{q}: \operatorname{Kerd}_{q}^{C} \rightarrow \operatorname{Kerd}_{q}^{D}$ Por 1.2.5 temos também que $\operatorname{Coker} d_{q}^{C}$ é um funtor, logo, $\operatorname{Im} d_{q+1}=\operatorname{Ker}\left(\operatorname{coker} d_{q+1}\right)$ é um funtor e temos um morfismo $\operatorname{Im} d_{q+1}^{C} \rightarrow \operatorname{Im} d_{q+1}^{D}$ Chame $\alpha_{q}^{C}: \operatorname{Im} d_{q+1}^{C} \rightarrow \operatorname{Kerd} d_{q}^{C}$, temos o seguinte diagrama de cokernel

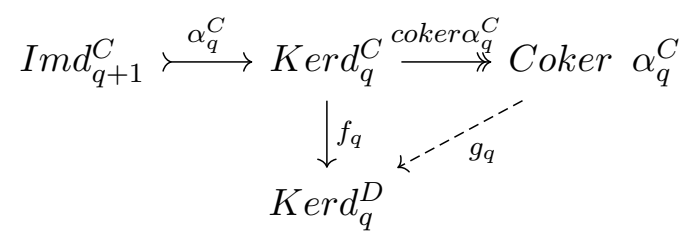

É claro que também existe um único morfismo $\alpha_{q}^{D}: \operatorname{Im} d_{q+1}^{D} \rightarrow \operatorname{Ker} d_{q}^{D}$, então existe um único $H_{q}\left(C_{\bullet}\right) \cong$ Coker $\alpha_{q}^{C} \rightarrow$ Coker $\alpha_{q}^{D} \cong H_{q}\left(D_{\bullet}\right)$

Pelo que vimos na seção de categorias abelianas temos que o morfismo $C_{q} \rightarrow C_{q-1}$ se fatora por um epi $C_{q} \rightarrow \operatorname{Imd}_{q} \cong \operatorname{Coimd}_{q}$.

Proposição 1.3.7 Seja $C$ • complexo de cadeia em uma categoria abeliana. Então o epimorfismo $C_{q} \rightarrow$ Imd $_{q}$ se fatora por um epimorfismo $p: C_{q} \rightarrow$ Cokerd $_{q+1}$ e temos $H_{q}\left(C_{\bullet}\right) \cong K \operatorname{erp}$

\section{Demonstração:}

A decomposição de $C_{q} \rightarrow \operatorname{Imd}_{q}$ por $p: C_{q} \rightarrow \operatorname{Cokerd}_{q+1}$ sai da definição de cokernel usando que $d_{q+1} \circ d_{q}=0$ e aplicando a decomposição em epi e mono no morfismo induzido Cokerd $_{q+1} \rightarrow C_{q-1}$, como ilustra o diagrama:

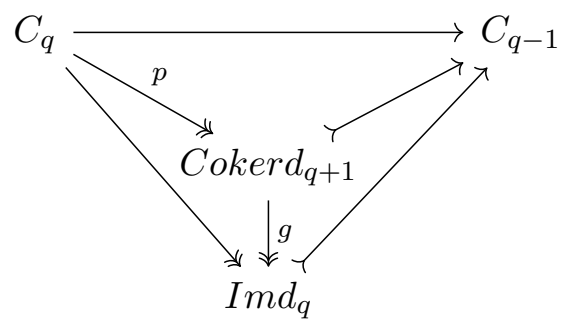

Agora vamos verificar o isomorfismo $H_{q}\left(C_{\bullet}\right) \cong K e r p$. Note que o diagrama abaixo é comutativo e as linhas são exatas (lembre que $\operatorname{Im} d_{q}=\operatorname{Coker}\left(k \operatorname{ker} d_{q}\right)$ ).

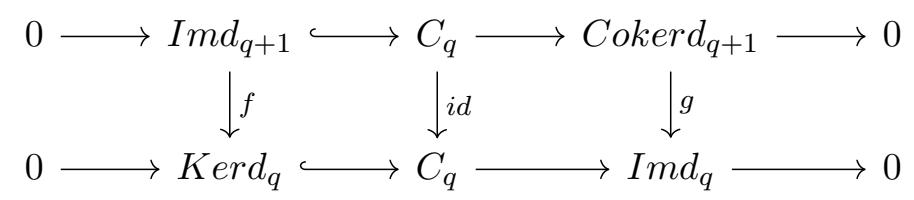

Pelo Lema da Serpente (1.1.25), existe $\partial: \operatorname{Kerp} \rightarrow \operatorname{Coker} f$ tal que a sequência

$$
0 \rightarrow \text { Kerf } \rightarrow \text { Kerid } \rightarrow \text { Kerg } \stackrel{\partial}{\rightarrow} \text { Coker } f \rightarrow \text { Cokerid } \rightarrow \text { Cokerg } \rightarrow 0
$$


é exata.

Lembre que para categorias abelianas Cokerf $=H_{q}\left(C_{\bullet}\right)$. Como Cokerid $=0=$ $\operatorname{Ker}(i d)$, concluímos que $\partial$ é um isomorfismo, pela Proposição 1.1.14.

Agora podemos demonstrar um teorema de amplo uso em Álgebra Homológica.

Teorema 1.3.8 Sejam $A_{\bullet}, B_{\bullet}, C_{\bullet}$ complexos em uma categoria abeliana $e 0 \rightarrow A \stackrel{f}{\rightarrow} B \stackrel{g}{\rightarrow}$ $C \rightarrow 0$ sequência exata curta. Então existem morfismos $\partial: H_{q}\left(C_{\bullet}\right) \rightarrow H_{q-1}\left(A_{\bullet}\right)$, chamados morfismos de conexão, tais que a sequência

$$
\ldots \longrightarrow H_{q+1}\left(C_{\bullet}\right) \stackrel{\partial}{\longrightarrow} H_{q}\left(A_{\bullet}\right) \longrightarrow H_{q}\left(B_{\bullet}\right) \longrightarrow H_{q}\left(C_{\bullet}\right) \stackrel{\partial}{\longrightarrow} \ldots
$$

é exata.

\section{Demonstração:}

Como $0 \rightarrow A \stackrel{f}{\rightarrow} B \stackrel{g}{\rightarrow} C \rightarrow 0$ é uma sequência exata, pelas proposições 1.2 .4 e 1.2.5, temos outras duas sequências exatas:

$$
\operatorname{Cokerd}_{q+1}^{A} \rightarrow \operatorname{Cokerd}_{q+1}^{B} \rightarrow \operatorname{Cokerd}_{q+1}^{C} \rightarrow 0
$$

e

$$
0 \rightarrow \operatorname{Kerd}_{q-1}^{A} \rightarrow \operatorname{Kerd}_{q-1}^{B} \rightarrow \operatorname{Kerd}_{q-1}^{C}
$$

E essas sequências se conectam por decomposições do tipo $\operatorname{Cokerd}_{q+1} \rightarrow \operatorname{Imd}_{q} \rightarrow$ $\operatorname{Ker} d_{q-1}$. De forma que nos deparamos com o seguinte diagrama, cujo retângulo central é comutativo:

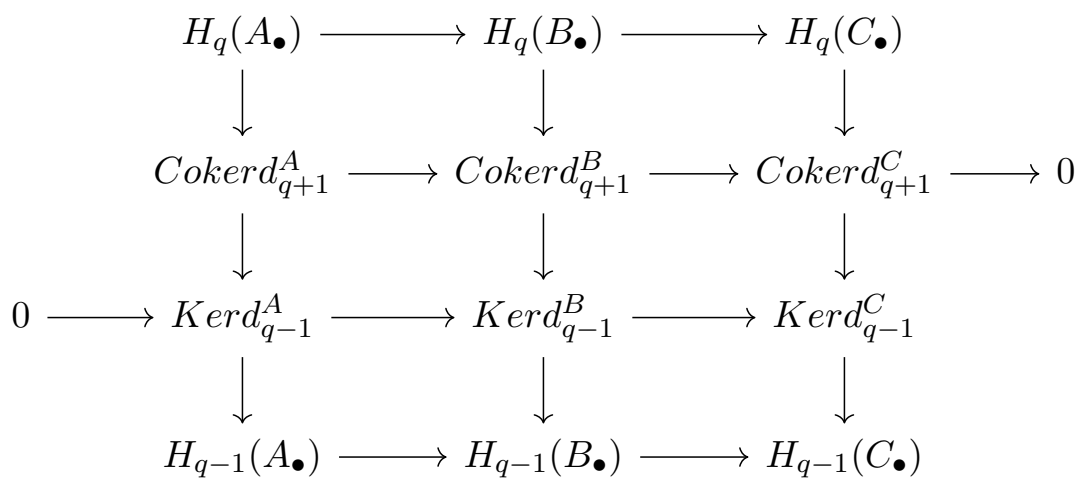

onde a linha de baixo vem simplesmente do $\operatorname{Coker}\left(\operatorname{Imd}_{q} \rightarrow \operatorname{Kerd} d_{q-1}\right)$ e para linha de cima usamos a Proposição 1.3.7.

Para concluir o Teorema basta aplicar o Lema da Serpente e temos que existe $\partial$ : $H_{q}\left(C_{\bullet}\right) \rightarrow H_{q-1}\left(A_{\bullet}\right)$ e a sequência longa induzida é exata.

Definição 1.3.9 Sejam $C_{\bullet}, D_{\bullet} \in C h(\mathscr{C})$ e $f_{\bullet}, g_{\bullet}: C_{\bullet} \rightarrow D_{\bullet}$ morfismos entres complexos. Dizemos que $f_{\bullet}$ e $g \bullet$ são homotópicas por cadeia se existe uma sequência de morfismos, chamada homotopia de cadeias $h_{q}: C_{q} \rightarrow D_{q+1}$ tal que $f_{q}-g_{q}=d_{q+1}^{D} \circ h_{q}+h_{q-1} \circ d_{q}^{C}$.

Usamos o seguinte diagrama (não comutativo) para ilustrar essa definição:

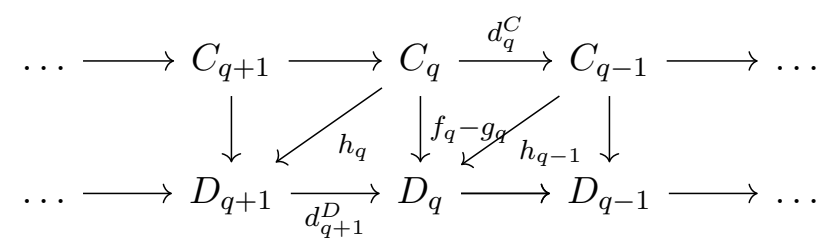

Notação: Quando dois morfismos $f, g$ são homotópicas por cadeia usamos $f \sim g$. 
Proposição 1.3.10 Se $f_{\bullet}, g_{\bullet}: C_{\bullet} \rightarrow D_{\bullet}$ são homotópicas por cadeia, então $H_{q}\left(f_{\bullet}\right)=$ $H_{q}\left(g_{\bullet}\right)$

\section{Demonstração:}

A rigor teríamos que usar aqui os pseudoelementos para demonstrar o resultado para uma categoria abeliana qualquer. Vamos apresentar como seria a demonstração desse resultado no caso de uma categoria abeliana concreta como $R-M o d$

Seja $h$ a homotopia entre $f_{\bullet}$ e $g_{\bullet}$. Dada $a \in H_{q}\left(C_{\bullet}\right)$ existe $[a] \in \operatorname{Kerd}_{q}^{C}$ (os colchetes denotam a classe) que representa $a$, veremos que $f_{q}([a]) \sim g_{q}([a])$, isto é, $f_{q}([a])-g_{q}([a])$ está em $\operatorname{Im} d_{q+1}^{D}$.

Sabemos que $d \circ h-h \circ d=f-g$. Aplicando $[a]$ :

$$
d_{q+1}^{D} \circ h_{q}([a])+h_{q-1} \circ d_{q}^{C}([a])=f_{q}([a])+g_{q}([a])
$$

Como $[a] \in \operatorname{Kerd}_{q}^{C}, d_{q+1}^{D} \circ h_{q}([a])=f_{q}([a])-g_{q}([a])$.

Então $f_{q}([a])-g_{q}([a]) \in \operatorname{Im} d_{q+1}^{D}, \operatorname{logo}, H_{q}\left(f_{\bullet}\right)=H_{q}\left(g_{\bullet}\right)$, como queríamos.

\subsection{Funtores Derivados}

Não é apenas o funtor $H_{q}(-)$ que produz sequências longas exatas a partir de sequências exatas curtas, da forma como vimos no Teorema 1.3.7. De forma geral os funtores que cumprem este papel são os funtores derivados.

Até o momento demos mais atenção para homologia pois o exemplos iniciais e o geométricos são de homologia, contudo, queremos trabalhar com Topos mais a frente e para os Topos o que vem naturalmente são os grupos de cohomologia, os quais coincidem com funtores derivados à direita. Nessa seção focaremos com objetos ligados à cohomologia, mas faremos comentários sobre suas versões duais atreladas à homologia. Mais uma vez seguimos [Wei95], além de [Har77] e [Pic02].

Definição 1.4.1 Seja $\mathscr{C}$ uma categoria abeliana e $I$ um objeto em $\mathscr{C}$, dizemos que $I$ é injetivo se satisfaz a seguinte propriedade: Dado um monomorfismo $f: A \rightarrow B$ e um morfismo $\alpha: A \rightarrow I$ existe pelo menos um $\beta: A \rightarrow I$ tal que $\alpha=\beta \circ f$. Diagramaticamente:

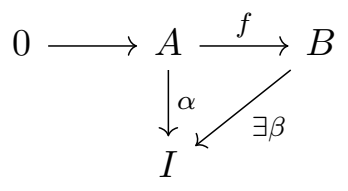

Dualmente definimos um objeto projetivo $P$ em $\mathscr{C}$ :

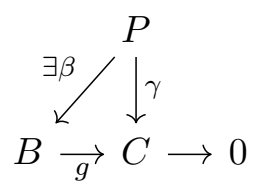

Note que um objeto $I$ em $\mathscr{C}$ é injetivo se $I$ é projetivo em $\mathscr{C}^{o p}$ e vale a recíproca, afinal $\mathscr{C}$ é abeliana se, e somente se, $\mathscr{C}^{o p}$ é abeliana . Consequentemente os resultados que veremos para objetos injetivos valem dualmente para os projetivos. Ainda, há uma outra caracterização possível, como se pode ver no seguinte lema.

Lema 1.4.2 Um objeto I em $\mathscr{C}$ é injetivo se, e só se, o funtor contravariante $\operatorname{Hom}_{\mathscr{C}}(-, I)$ é exato, isto é, se para cada sequência exata curta $0 \rightarrow A \rightarrow B \rightarrow C \rightarrow 0$ em $\mathscr{C}$ temos uma sequência exata curta em $\boldsymbol{A b}$ dada por $0 \rightarrow \operatorname{Hom}_{\mathscr{C}}(C, I) \rightarrow \operatorname{Hom}_{\mathscr{C}}(B, I) \rightarrow$ $\operatorname{Hom}_{\mathscr{C}}(A, I) \rightarrow 0$ 


\section{Demonstração:}

Seja $0 \rightarrow A \stackrel{f}{\rightarrow} B \stackrel{g}{\rightarrow} C \rightarrow 0$ exata em $\mathscr{C}$.

$(\Longrightarrow)$ Suponha $I$ injetivo. Sabemos que $\operatorname{Hom}_{\mathscr{C}}(-, I)$ é funtor exato à esquerda (Proposição 1.2.3), então basta mostrar que $f_{*}: \operatorname{Hom}_{\mathscr{C}}(B, I) \rightarrow \operatorname{Hom}_{\mathscr{C}}(A, I)$ é sobrejetora.

Como $I$ é injetivo e $f$ é mono, dado $\alpha \in \operatorname{Hom}_{\mathscr{C}}(A, I)$ existe $\beta \in \operatorname{Hom}_{\mathscr{C}}(B, I)$ tal que $\alpha=\beta f=f_{*} \beta, \operatorname{logo}, f_{*}$ é sobrejetora.

$(\Longleftarrow)$ Suponha $\operatorname{Hom}_{\mathscr{C}}(-, I)$ exato. Seja $\alpha \in \operatorname{Hom}_{\mathscr{C}}(A, I)$, ou seja, $\alpha: A \rightarrow I$ morfismo em $\mathscr{C}$.

Como $0 \rightarrow A \stackrel{f}{\rightarrow} B \stackrel{g}{\rightarrow}$ Coker $f \rightarrow 0$ exata e $\operatorname{Im} f \cong \operatorname{Ker}($ Coker $f)$, tome $C=$ Coker $f$. Temos que a sequência $0 \rightarrow \operatorname{Hom}_{\mathscr{C}}(C, I) \stackrel{g_{*}}{\rightarrow} \operatorname{Hom}_{\mathscr{C}}(B, I) \stackrel{f_{*}}{\rightarrow} \operatorname{Hom}_{\mathscr{C}}(A, I) \rightarrow 0$ é exata, $\operatorname{logo}, f_{*}$ é sobrejetora. Então existe $\beta \in \operatorname{Hom}_{\mathscr{C}}(B, I)$ tal que $\alpha=f_{*} \beta=\beta f$. Assim concluímos que $I$ é injetivo.

Proposição 1.4.3 Sejam $I_{1}$ e $I_{2}$ objetos em $\mathscr{C}$, então $I_{1} \oplus I_{2}$ é injetivo se, e só se, $I_{1} e$ $I_{2}$ são injetivos.

\section{Demonstração:}

$(\Longrightarrow)$ Seja $I_{1} \oplus I_{2}$ injetivo, vamos mostrar que $I_{1}$ é injetivo e, por simetria, vemos que $I_{2}$ é injetivo.

Sejam $f: A \rightarrow B$ um mono e $\alpha: A \rightarrow I_{1}$ morfismo qualquer. Considere $s_{1}: I_{1} \rightarrow I_{1} \oplus I_{2}$, como na Proposição 1.1.10. Já que $I_{1} \oplus I_{2}$ é injetivo, existe $\beta$ tal que $s_{1} \circ \alpha=\beta \circ f$. Agora compomos com a projeção: $p_{1} \circ s_{1} \circ \alpha=p_{1} \circ \beta \circ f$. Desta forma $p_{1} \circ \beta: B \rightarrow I_{1}$ é tal que $\alpha=p_{1} \circ \beta \circ f$, ou seja, $I_{1}$ é injetivo.

$(\Longleftarrow)$ Sejam $I_{1}$ e $I_{2}$ injetivos. Considere $f: A \rightarrow B$ mono e $\alpha: A \rightarrow I_{1} \oplus I_{2}$. Como $I_{1}$ é injetivo, considerando dado $p_{1} \circ \alpha: A \rightarrow I_{1}$, onde $p_{1}$ é a projeção, temos que existe $\beta_{1}$ tal que $p_{1} \circ \alpha=\beta_{1} \circ f$ e repetimos o argumento para $I_{2}$. Usando a propriedade do produto obtemos $\beta: B \rightarrow I_{1} \oplus I_{2}$ que faz o seguinte diagrama comutar:

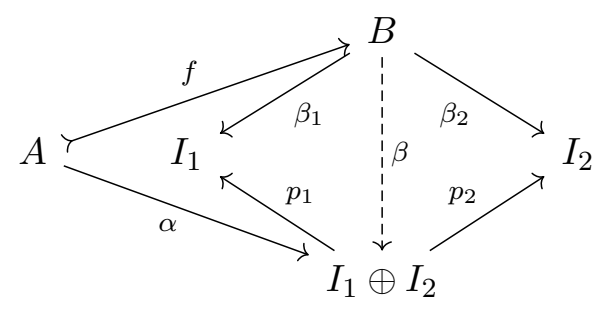

Portanto, $I_{1} \oplus I_{2}$ é injetivo.

Note que esse lado vale em geral para qualquer família $\left\{I_{j} \mid j \in J\right\}$.

Definição 1.4.4 Seja $A \in \mathscr{C}$ e $I^{\bullet} \in \mathbf{C h}(\mathscr{C})$ com $I^{i}=0$ para todo $i<0$ e seja $\mathcal{E}: A \rightarrow I$ morfismo. Dizemos que $I^{\bullet}$ é uma resolução de $A$ se a seguinte sequência é exata.

$$
0 \longrightarrow A \stackrel{\mathcal{E}}{\longrightarrow} I^{0} \longrightarrow I^{1} \longrightarrow I^{2} \longrightarrow \ldots
$$

Se ainda tivermos que $I^{i}$ é injetivo para todo $i$, chamamos de resolução injetiva.

Podemos abreviar a sequência acima por $0 \longrightarrow A \stackrel{\mathcal{E}}{\longrightarrow} I^{\bullet}$

Definição 1.4.5 Dizemos que uma categoria $\mathscr{C}$ possui suficientes objetos injetivos se para todo $A$ em $\mathscr{C}$ existe um monomorfismo $A \rightarrow I$ com $I$ injetivo.

Observe que se $\mathscr{C}$ possui suficientes objetos injetivos, então todo objeto $A$ de $\mathscr{C}$ admite resolução injetiva. 
Teorema 1.4.6 (Teorema da Comparação) Seja $J^{\bullet}$ resolução injetiva de $B$ e $f$ : $A \rightarrow B$ um morfismo em $\mathscr{C}$. Então, para toda resolução $I^{\bullet}$ de A existe um morfismo de complexos (único a menos de equivalência homotópica) $f^{\bullet}: I^{\bullet} \rightarrow J^{\bullet}$ tal que o seguinte diagrama comuta

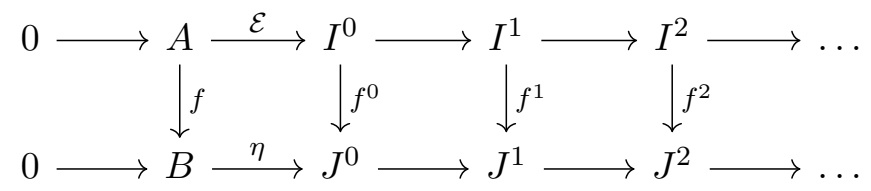

\section{Demonstração:}

Faremos uma prova por indução. ${ }^{3}$ Considere a composição $\eta \circ f: A \rightarrow J^{0}$. Como $J^{0}$ é injetivo, existe um morfismo de $I^{0} \rightarrow J^{0}$, o qual iremos chamar de $f^{0}$. Agora supomos que $f^{n}: I^{n} \rightarrow J^{n}$ existe e vamos encontrar $f^{n+1}$.

Temos $d_{I}^{n}: I^{n} \rightarrow I^{n+1}$, como estamos em categorias abelianas, esse morfismo admite uma fatora em epi e mono da forma

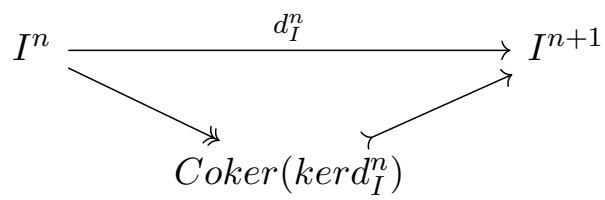

Sendo que $\operatorname{Coker}\left(k e r d_{I}^{n}\right) \cong \operatorname{Im}_{I}^{n}$. Mas $I^{\bullet}$ é um resolução, então $I^{n} \stackrel{d_{I}^{n}}{\longrightarrow} I^{n+1} \stackrel{d_{I}^{n+1}}{\longrightarrow} I^{n+2}$ é exata e, portanto, $\operatorname{Im} d_{I}^{n}=\operatorname{Ker} d_{I}^{n+1}$.

Chame o morfismo de $I^{n} \rightarrow J^{n+1}$ de $g=d_{J}^{n} \circ f^{n}$, usando a propriedade universal do cokernel e considere o cokernel $\operatorname{Coker}\left(k e r d_{I}^{n}\right) \cong \operatorname{Kerd}_{I}^{n+1}$ temos um diagrama comutativo

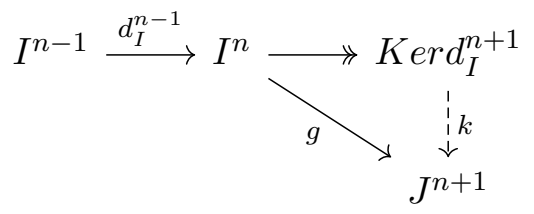

Como $J^{n+1}$ é injetivo, o diagrama $0 \longrightarrow \operatorname{Kerd}_{I}^{n+1} \succ I_{\substack{J^{n+1} \\ f^{n+1}}}$ comuta,

onde $\operatorname{Kerd}_{I}^{n+1} \longmapsto I^{n+1}$ é o mono obtido da decomposição em epi e mono de $d_{I}^{n}$.

Assim garantimos que $f^{n+1}$ existe, isto é, que o morfismo entre complexos $f^{\bullet}$ existe. Agora vamos supor que existe outro mosfismo de cadeias $f^{\prime \bullet}$ e e encontrar uma homotopia entre $f^{\bullet}$ e $f^{\prime \bullet}$ para provar a unicidade. Construiremos essa homotopia $h$ também por indução.

Podemos supor $f^{0}=0$ (apenas para facilitar as contas, mas não há surpresas para o caso geral), dessa forma queremos homotopias $h^{n}: I^{n} \rightarrow J^{n-1}$ que garantam que $f^{\bullet}$ é homotópico a 0 . No caso $n=0$ basta tomarmos $h^{0}=0$ e temos o primeiro passo da indução.

Suponha agora que vale $f^{n}=h^{n+1} \circ d_{I}^{n}+d_{J}^{n-1} \circ h^{n}$, vamos encontrar $h^{n}$. Isolamos $f^{n}-d_{J}^{n-1} \circ h^{n}$ e compomos com $d^{n-1}$. Assim:

\footnotetext{
${ }^{3}$ Esta pode ser formalizada com o uso adequado do axioma da escolha.
} 


$$
\begin{aligned}
\left(f^{n}-d_{J}^{n-1} \circ h^{n}\right) \circ d_{I}^{n-1} & =f^{n} \circ d_{I}^{n-1}-d_{J}^{n-1} \circ h^{n} \circ d_{I}^{n-1} \\
& =f^{n} \circ d_{I}^{n-1}-d_{J}^{n-1} \circ\left(f^{n-1}-d_{J}^{n-2} \circ h^{n-1}\right) \\
& =f^{n} \circ d_{I}^{n-1}-d_{J}^{n-1} \circ f^{n-1} \\
& =0
\end{aligned}
$$

A segunda linha vale pela hipótese de indução, na terceira usamos que $d_{J}^{n-1} \circ d_{J}^{n-2}=0$ e a igualdade final foi obtida pela comutatividade do diagrama.

Isso significa que temos uma decomposição $I^{n} \rightarrow \operatorname{Cokerd}^{n-1} \rightarrow I^{n+1} \rightarrow I^{n+1}$.

De forma similar ao que fizemos anteriormente, os diagramas abaixo comutam
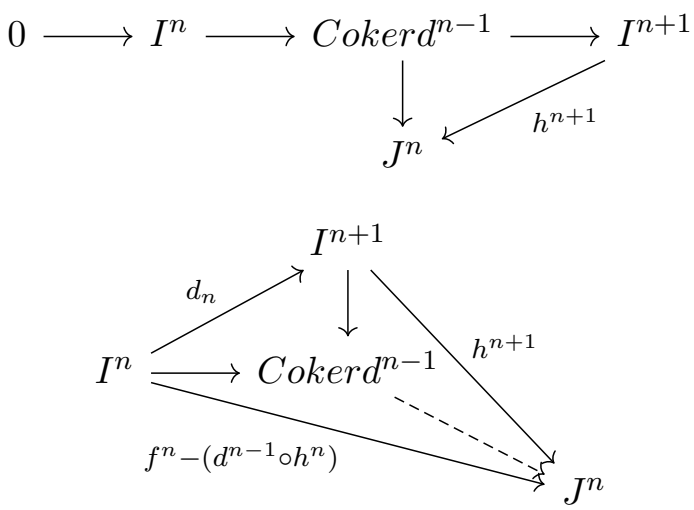

Assim, $f^{n}-d^{n-1} \circ h^{n}=h^{n+1} \circ d^{n}$.

Observação 1.4.7 No diagrama abaixo, as linhas são exatas se, e só se, as sequências diagonais são exatas, onde os objetos $X^{i}$ são tais que os morfismos $I^{i-1} \rightarrow I^{i}$ são decompostos em epis e monomorfismos.

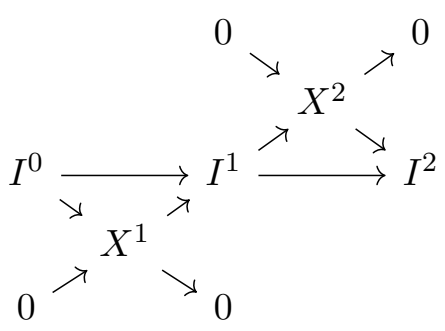

Veja que essa observação é consequência da Proposição 1.1.24

Com isso podemos pensar em sequências exatas como várias sequências exatas curtas que se cruzam, em particular, seria uma forma de enxergar uma resolução. Expandindo o diagrama acima obtemos:

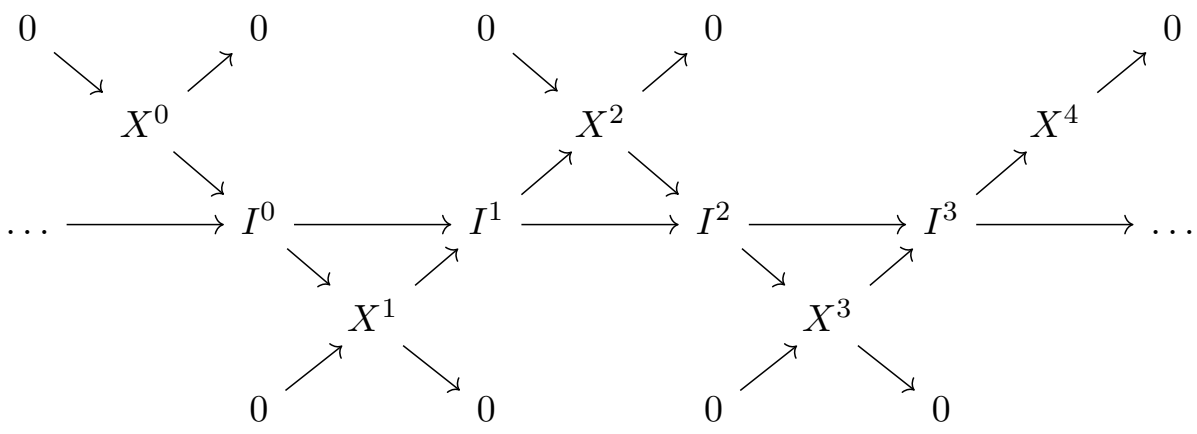

Vamos mostrar mais um resultado bastante conhecido sobre resoluções injetivas: 
Teorema 1.4.8 Seja $\mathscr{C}$ uma categoria com suficientes objetos injetivos. Se o diagrama em $\mathscr{C}$ dado por

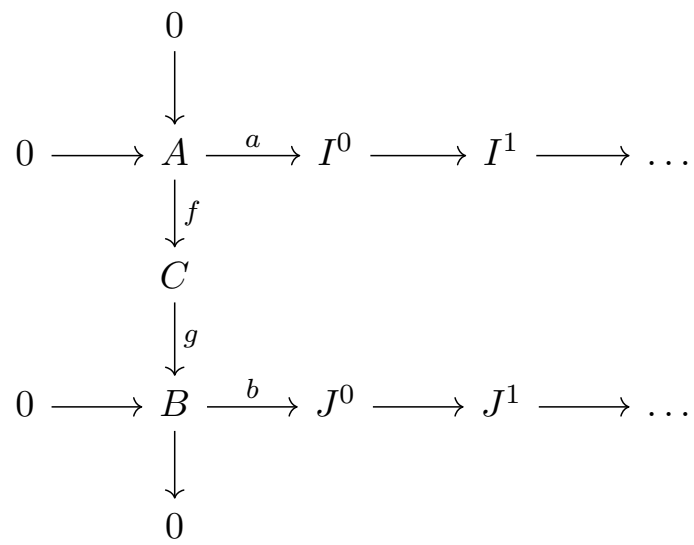

é tal que a coluna é uma sequência exata curta e as linhas são resoluções injetivas de $A$ e $B$, repectivamente. Então podemos completá-lo para um diagrama da forma

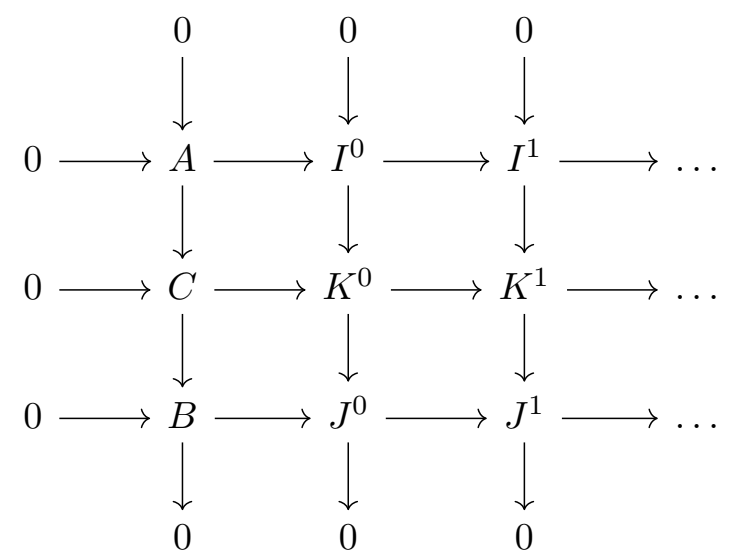

onde todas as colunas são exatas, a linha do meio é uma resolução injetiva de $C$ e $K^{n}=I^{n} \oplus J^{n}$ para todo $n \geq 0$.

\section{Demonstração:}

Vamos chamar os morfismos de $I^{n} \rightarrow I^{n+1}$ de $d_{I}^{n}$

Podemos tomar o cokernel de $a$ e $b$ para obter sequências exatas curtas da forma $0 \rightarrow A \stackrel{a}{\rightarrow} I^{0} \rightarrow$ Coker $\rightarrow 0$ e $0 \rightarrow B \stackrel{b}{\rightarrow} J^{0} \rightarrow$ Coker $b \rightarrow 0$, assim passamos a considerar o seguinte diagrama.

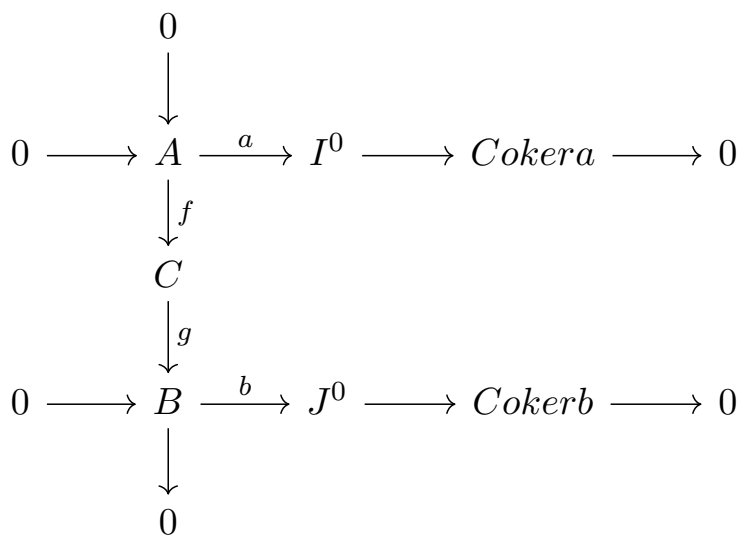

Pela propriedade do produto, os morfismos $\beta: C \rightarrow I^{0}$ tal que $\beta \circ f=a$ (que existe pois $I^{0}$ é injetivo) e $b \circ g$ induzem um morfismo $c: C \rightarrow I^{0} \oplus J^{0}$. 
Chame $s: I^{0} \rightarrow I^{0} \oplus J^{0}$, então as projeções fornecem: $p_{I^{0}} \circ c \circ f=\beta \circ f=a=p_{I^{0}} \circ s \circ a$ e $p_{J^{0}} \circ c \circ f=b \circ g \circ f=0=p_{J^{0}} \circ s \circ a$. Pela propriedade do produto, $c \circ f=q \circ a$. Pela contrução de $c$ encontramos $b \circ g=p_{J^{0}} \circ c$.

Agora é só aplicar o Lema da Serpente para obter que o seguinte diagrama comuta, cujas linhas e colunas são exatas.

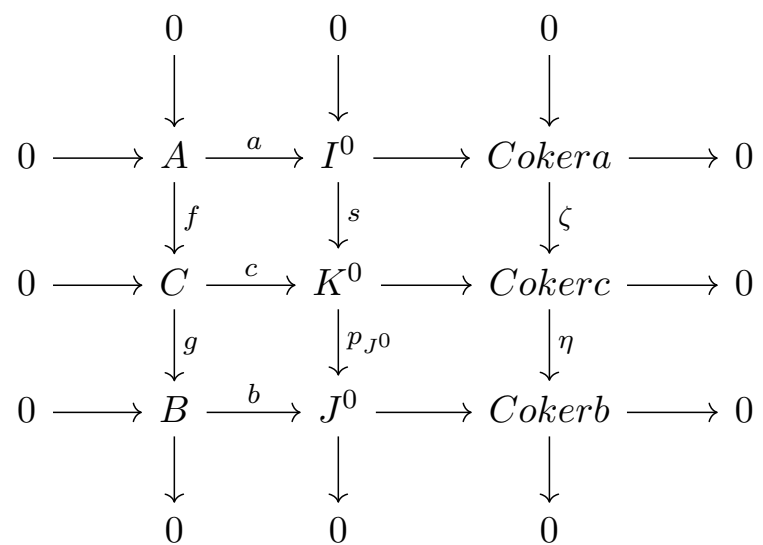

Note que a exatidão de $0 \rightarrow$ Coker $a \rightarrow$ Cokerb $\rightarrow$ Cokerc $\rightarrow 0$ também sai do Lema de Serpente, pois existe um morfirmos $\partial$ que conecta Kerb e Cokera e, como $0 \rightarrow B \stackrel{b}{\rightarrow}$ $J^{0} \rightarrow$ Cokerb $\rightarrow 0$ é exata curta, Kerb $=0$.

Aplicamos a mesma ideia acima na terceira coluna do último diagrama obtido. Indutivamente, a partir de

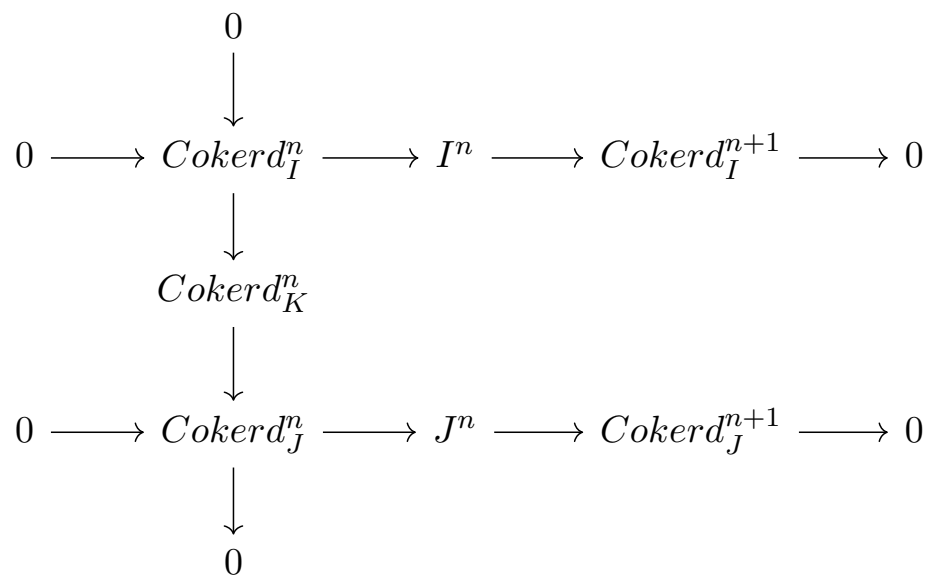

obtemos

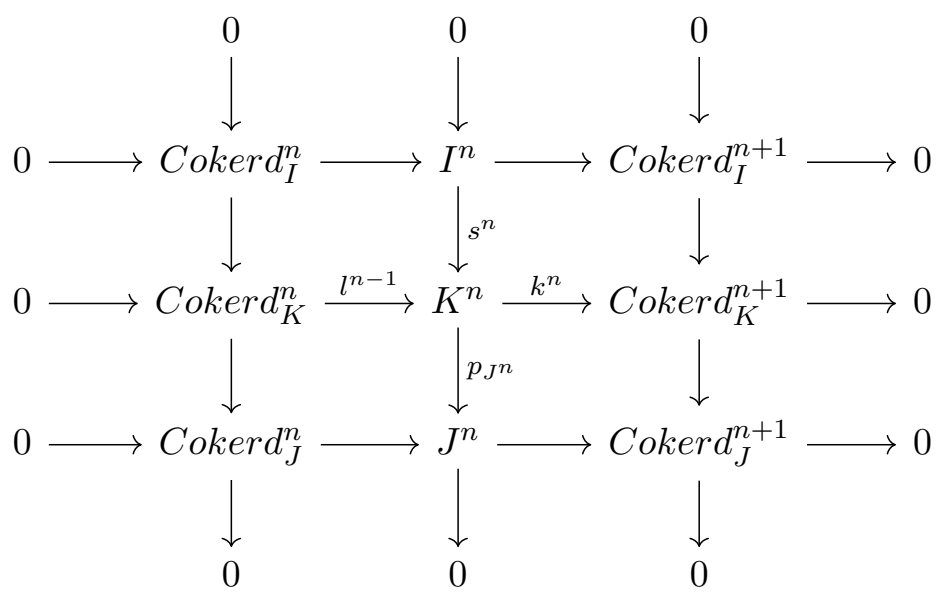

Fazendo $d_{K}^{n}=l^{n} \circ k^{n}$ temos a resolução $K^{\bullet}$ desejada. Pela Proposição 1.4.3, é injetiva e assim concluímos a demonstração do Teorema. 
Teorema 1.4.9 Sejam $\mathscr{C}$ e $\mathscr{C}^{\prime}$ categorias abelianas, sendo $\mathscr{C}$ com suficientes objetos injetivos e $F: \mathscr{C} \rightarrow \mathscr{C}^{\prime}$ funtor exato à esquerda. Então:

(i) Existem funtores aditivos $R^{q} F: \mathscr{C} \rightarrow \mathscr{C}^{\prime}$ para todo $q \geq 0$;

(ii) $F \cong R^{0} F$ é um isomorfismo;

(iii) Para cada sequência exata $E: 0 \rightarrow A_{1} \rightarrow A_{2} \rightarrow A_{3} \rightarrow 0$ e para cada $q \geq 0$, existe um morfismo natural $\delta_{E}^{q}: R^{q} F A_{3} \rightarrow R^{q+1} F A_{1}$ que torna a sequência a seguir exata

$$
\cdots \rightarrow R^{q} F A_{1} \rightarrow R^{q} F A_{2} \rightarrow R^{q} F A_{3} \stackrel{\delta_{E}^{q}}{\longrightarrow} R^{q+1} F A_{1} \rightarrow \ldots
$$

(iv) Os morfismo $\delta_{E}^{q}$ são naturais em $E$.

\section{Demonstração:}

Não custa lembrar que para um funtor ser exato à esquerda ele precisa ser aditivo. A propriedade de ser aditivo será mais utilizada do que a de ser exato à esquerda nessa demonstração.

(i) Seja $A$ um objeto qualquer de $\mathscr{C}$ e considere uma resolução injetiva $0 \stackrel{\mathcal{E}_{A}}{\longrightarrow} I_{A}^{\bullet}$. Temos que $F\left(I_{A}^{\bullet}\right)$ é um complexo de cadeia em $\mathscr{C}^{\prime}$ e com isso definimos, provisoriamente,

$$
R^{q} F(A)=H^{q} F\left(I_{A}^{\bullet}\right)
$$

Seja $f: A \rightarrow B$ um morfismo em $\mathscr{C}$

Pelo Teorema 1.4.6 existe um único morfismo, a menos de homotopia, $f^{\bullet}: I_{A}^{\bullet} \rightarrow I_{B}^{\bullet}$ tal que o diagrama abaixo comuta

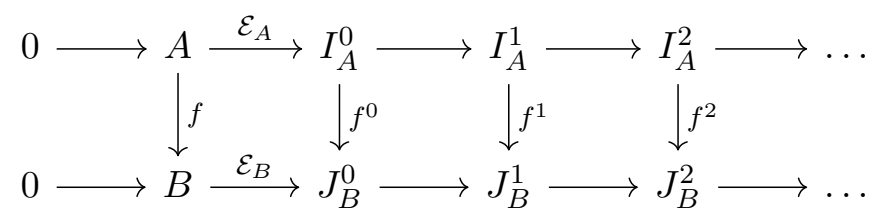

Agora defina provisoriamente $R^{q} F(f)=H^{q} F\left(f^{\bullet}\right)$. Como $F$ é aditivo, ele preserva homotopias. Então, pela Proposição 1.3 .10 e por $f^{\bullet}$ ser único a menos de homotopia, garantimos que $H^{q} F\left(f^{\bullet}\right)$ não depende da escolha de $f^{\bullet}$.

Antes de prosseguirmos devemos mencionar que estamos deixando passar alguns detalhes. Considere duas resoluções injetivas $0 \rightarrow A \stackrel{\mathcal{E}}{\rightarrow} I^{\bullet}$ e $0 \rightarrow A \stackrel{\mathcal{E}^{\prime}}{\rightarrow} I^{\prime \bullet}$ com morfismos de complexos (como já mencionamos, únicos a menos de homotopia) $f^{\bullet}$ : $I^{\bullet} \rightarrow I^{\prime \bullet}$ e $f^{\prime \bullet}: I^{\bullet \bullet} \rightarrow I^{\bullet}$. Assim, $f^{\prime \bullet} \circ f^{\bullet} \sim i d_{I^{\bullet}}$ e $f^{\bullet} \circ f^{\prime \bullet} \sim i d_{I^{\prime}}$. Então $R^{q}(f)$ e $R^{q}\left(f^{\prime}\right)$ são isomorfismos inversos, por isso dizemos que não depende da escolhar de $f^{\bullet}$, mas não garantimos ser a identidade de $R^{q} F(A)$.

Isso significa que a definição $R^{q} F(A)=H^{q} F\left(I_{A}^{\bullet}\right)$ não funciona perfeitamente. Fixada a resolução ela cumpre um papel satisfatório, mas se quisermos verificar de fato que a escolha da resolução não interfere em $H^{q} F\left(f^{\bullet}\right)$ e que $R^{q} F$ é funtor, precisarimos de truques categoriais. Mais precisamente, o que temos no momento é que $R^{F}(A) \cong$ $H^{q} F\left(I_{A}^{\bullet}\right)$ mas seguiremos sendo superficiais em relação a esse tipo de detalhe técnico (importante, mas excessivamente trabalhoso. Na seção 5 do Capítulo IV de [HS71] tem mais detalhes sobre isso. Apesar do foco ser em funtores derivados à esquerda para a categoria dos $R$-Mod, o procedimento é essencialmente o mesmo). Voltando para a demonstração: 
Considere um outro morfismo em $\mathscr{C}, g: B \rightarrow C$. Podemos aplicar o Teorema 1.4.6 na composição $g \circ f$ e obter um morfismo de complexos $g^{\bullet} \circ f^{\bullet}$.Assim,

$$
R^{q} F(g f)=H^{q} F\left(g^{\bullet} \circ f^{\bullet}\right)=\left(H^{q} F\left(g^{\bullet}\right)\right)\left(H^{q} F\left(f^{\bullet}\right)\right)=\left(R^{q} F(g)\right)\left(R^{q} F(f)\right)
$$

e, é claro,

$$
R^{q} F\left(i d_{A}\right)=H^{q} F\left(i d_{I_{A}^{\bullet}}\right)=i d_{H}^{q}\left(F\left(I_{A}^{\bullet}\right)\right.
$$

Então $R^{q} F$ é um funtor.

Para a aditividade note que um morfismo da forma $f+f^{\prime}$ induz um morfismo de cadeia $f^{\bullet}+f^{\prime \bullet}$. Por hipótese $F$ é aditivo e usando que $H^{q}$ é aditivo (não provamos isso, mas não é difícil verificar) obtemos

$$
R^{q} F\left(f+f^{\prime}\right)=H^{q} F\left(f^{\bullet}+f^{\bullet}\right)=H^{q} F\left(f^{\bullet}\right)+H^{q} F\left(f^{\bullet}\right)=R^{q} F(f)+R^{q} F\left(f^{\prime}\right)
$$

Portanto, $R^{q} F: \mathscr{C} \rightarrow \mathscr{C}^{\prime}$ é aditivo para todo $q \geq 0$

(ii) Temos que a sequência $0 \rightarrow A \stackrel{\mathcal{E}_{A}}{\rightarrow} I_{A}^{0} \stackrel{d^{0}}{\rightarrow} I_{A}^{1}$ é exata em $\mathscr{C}$. Como $F$ é funtor exato à esquerda, então $0 \rightarrow F A \stackrel{F \mathcal{E}_{A}}{\longrightarrow} F I_{A}^{0} \stackrel{F d^{0}}{\longrightarrow} F I_{A}^{1}$ é exata em $\mathscr{C}^{\prime}$. Como $I m F d^{-1}=0$, então existe um isomorfismo $H^{0}\left(F I_{A}^{\bullet}\right) \cong \operatorname{Ker}\left(F d^{0}\right)$.

Pela propriedade universal do kernel o seguinte diagrama comuta

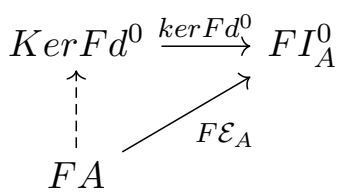

Como a sequência em $\mathscr{C}^{\prime}$ é exata, então $F \mathcal{E}_{A}=k e r F d^{0}$. Logo, existe um isomorfismo $k: F A \rightarrow H^{0} F_{A}^{\bullet}=R^{0} F A$, como queríamos.

(iii) Seja $E: 0 \rightarrow A_{1} \rightarrow A_{2} \rightarrow A_{3} \rightarrow 0$ uma sequência exata em $\mathscr{C}$. Aplicando o Teorema 1.4.8 encontramos uma resolução injetiva $0 \rightarrow A_{2} \stackrel{\mathcal{E}_{A_{2}}}{\longrightarrow} K^{\bullet}$ além de sequências exatas $0 \rightarrow I^{\bullet} A_{1} \stackrel{q^{\bullet}}{\longrightarrow} K^{\bullet} \stackrel{p^{\bullet} I A_{3}}{\longrightarrow} I^{\bullet} A_{3}$, sendo que a exatidão dessas sequências é mera consequência das decomposições da soma direta (lembre que $K=I_{A_{1}} \oplus I_{A_{3}}$ ). Dessa forma, como $F$ é aditivo e usando que não dependemos da escolha da resolução injetiva, conseguimos sequências exatas $0 \rightarrow F I^{\bullet} A_{1} \stackrel{F q^{\bullet}}{\longrightarrow} F K^{\bullet} \stackrel{F p^{\bullet} A_{3}}{\longrightarrow} F I^{\bullet} A_{3} \rightarrow 0$, onde $F K^{\bullet}=F\left(I_{A_{1}}^{\bullet}\right) \oplus F\left(I_{A_{3}}^{\bullet}\right)$

Pelo Teorema 1.3.8 existem morfismos de conexão que fornecem sequências exatas longas, já usando a definição dos funtores $R^{q} F$ obtemos:

$$
\cdots \rightarrow R^{q} F A_{1} \rightarrow H^{q} K^{\bullet} \rightarrow R^{q} F A_{3} \stackrel{\delta^{q}}{\rightarrow} R^{q+1} F A_{1} \rightarrow \ldots
$$

Definimos o morfismo desejado por $\delta_{E}^{q}=\delta^{q}$. Novamente, podemos fazer isso porque $\delta^{q}$ não depende da escolha da resolução $K^{\bullet}$. Veja, abrindo um pouco mais os comentários já feitos, mas ocultando ainda alguns detalhes, se tomarmos $J^{\bullet}$ uma outra resolução de $A_{2}$ conseguimos um diagrama comutativo como o seguinte: 


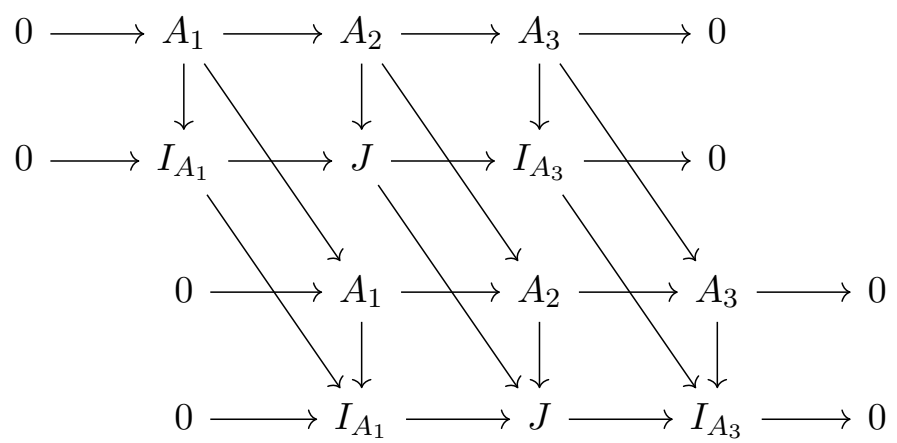

Aplicamos $F$ nas sequências exatas curtas e obtemos novas sequências exatas que gerantem que

$$
\cdots \rightarrow R^{q} F A_{1} \rightarrow H^{q} J^{\bullet} \rightarrow R^{q} F A_{3} \stackrel{\delta^{q}}{\rightarrow} R^{q+1} F A_{1} \rightarrow \ldots
$$

é também exata.

Agora vamos verificar que $H^{q} K^{\bullet} \cong R^{q} F A_{2}$ é um isomorfismo. Considere o diagrama comutativo

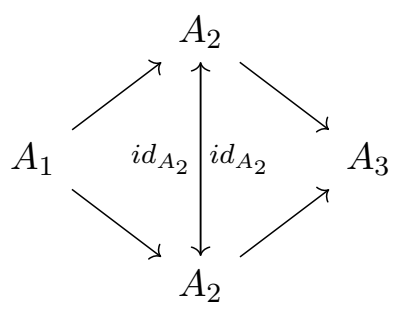

O Teorema 1.4.6 nos fornece morfismos entre complexos e garante a comutatividade (a menos de homotopia) do diagrama

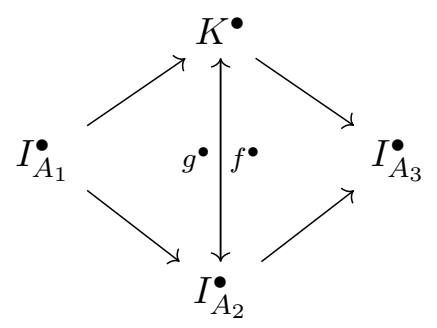

onde $K^{\bullet}$ é a resolução de $I_{A_{2}}$ construída acima e $I_{A_{2}}^{\bullet}$ é outra resolução de $I_{A_{2}}$. Assim, $f^{\bullet} \circ g^{\bullet} \sim i d_{I_{A_{2}}}$ e $g^{\bullet} \circ f^{\bullet} \sim i d_{K} \bullet$

Tanto $F$ quando $H^{q}$ são funtores aditivos, portanto, podemos aplicá-los nesse diagrama e preservar a comutatividade do diagrama. Em particular, $H^{q} F\left(f^{\bullet}\right)$ e $H^{q} F\left(g^{\bullet}\right)$ são inversos um do outro e usando a Proposição 1.3.10 são isos. Portanto,

$$
\cdots \rightarrow R^{q} F A_{1} \rightarrow H^{q} K^{\bullet} \rightarrow R^{q} F A_{3} \stackrel{\delta^{q}}{\rightarrow} R^{q+1} F A_{1} \rightarrow \ldots
$$

gera

$$
\cdots \rightarrow R^{q} F A_{1} \rightarrow R^{q} F A_{2} \rightarrow R^{q} F A_{3} \stackrel{\delta_{E}^{q}}{\longrightarrow} R^{q+1} F A_{1} \rightarrow \ldots
$$

(iv) Vamos indicar o caminho exibindo os diagramas, mas não vamos mostrar em detalhes. É mais fácil entender o que está acontecendo assim do que escrever cada passagem. Considere duas sequências exatas curtas $0 \rightarrow A_{1} \rightarrow A_{2} \rightarrow A_{3} \rightarrow 0$ e $0 \rightarrow B_{1} \rightarrow$ $B_{2} \rightarrow B_{3} \rightarrow 0$ tais que o diagrama abaixo comuta 


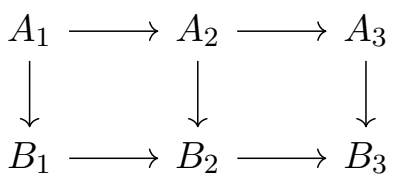

Pelos itens $(i)$ e $(i i i)$ temos que os dois primeiros quadrados do diagrama abaixo comutam

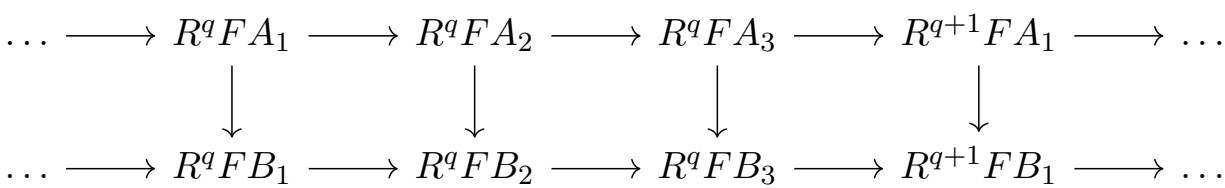

É possível verificar que os quadrados da forma $\left.\right|_{R^{q} F B_{3} \longrightarrow R^{q+1} F B_{1}} ^{R^{q} F A_{3} \longrightarrow R^{q+1} F A_{1}}$ também comumta e assim concluímos a naturalidade de $\delta$.

Definição 1.4.10 Os funtores $R^{q} F: \mathscr{C} \rightarrow \mathscr{C}^{\prime}$ da prova do Teorema 1.4 .12 são ditos funtores derivados à direita, isto é, seja $F$ funtor exato à esquerda e $I$ uma resolução de $A$, definimos o q-ésimo funtor derivado à direita de $\mathbf{F}$ :

$$
R^{q} F(A) \cong H^{q} F\left(I^{\bullet}\right)
$$

Observe que se $F$ também for exato à direita então $H^{q} F\left(I^{\bullet}\right)=0$ para todo $q>0$.

Dualmente contruímos funtores derivados à esquerda e os denotamos por $L_{n} G$, onde $G$ é funtor exato à direita.

Definição 1.4.11 Seja $F: \mathscr{C} \rightarrow \mathscr{C}^{\prime}$ como no Teorema anterior e $I$ um objeto de $\mathscr{C}$. Dizemos que $I$ é $F$-acíclico (ou acíclico para $F$ ) se $R^{q} F(I)=0$ para todo $q>0$.

Observação 1.4.12 Note que se $I$ for um objeto injetivo de $\mathscr{C}$, então $0 \rightarrow I \stackrel{i d}{\rightarrow} I \rightarrow 0 \rightarrow$ $0 \rightarrow \ldots$ é uma resolução injetiva de $I$ e obtemos $R^{q} F(I) \cong H^{q} F\left(I^{\bullet}\right)=0$, para todo $q>0$.

O próximo resultado estabelece a relação entre os objetos de cohomologia e o funtor derivado à direta.

Teorema 1.4.13 Seja $F: \mathscr{C} \rightarrow \mathscr{C}^{\prime}$ como no Teorema 1.3.12 e suponha que exista uma sequência exata $0 \rightarrow A \rightarrow I^{0} \rightarrow I^{1} \rightarrow \ldots$ onde cada $I^{n}$ é $F$-acíclico para $n \geq 0$. Então $R^{q}(F(A)) \cong H^{q}\left(F\left(I^{\bullet}\right)\right)$, para todo $q \geq 0$.

\section{Demonstração:}

Como $I^{n}$ é $F$-acíclico para todo $q \geq 0$, pela Observação 1.4.7, temos diversas sequências exatas curtas que cruzam a resolução, tais sequências são da forma $0 \rightarrow X^{n} \rightarrow I^{n} \rightarrow$ $X^{n+1} \rightarrow 0$, onde $A \cong X^{0}$ é isomorfismo.

Pelo Teorema 1.4.9 cada uma dessas sequências induzem sequências exatas longas, mas cada $R^{q} F I^{n}=0, \forall q>0, n \geq 0$, logo, elas são do tipo:

$$
\ldots \stackrel{\cong}{\longrightarrow} R^{q} F X^{n} \longrightarrow 0 \longrightarrow R^{q} F X^{n+1} \stackrel{\cong}{\longrightarrow} R^{q+1} F X^{n} \longrightarrow 0 \longrightarrow \ldots
$$

Ou seja, $R^{q} F X^{n+1} \cong R^{q+1} F X^{n}, \forall q \geq 0, n \geq 0$ 
Então, além de $R^{0} F \cong F$, temos $R^{1} F I^{n-1}=0, \forall n>0$. Assim, a seguinte sequência é exata:

$$
0 \longrightarrow F X^{n-1} \longrightarrow F I^{n-1} \cong R^{0} F I^{n-1} \longrightarrow F X^{n} \longrightarrow R^{1} F X^{n-1} \longrightarrow 0
$$

Logo, $R^{q} F A \cong R^{q} F X^{0} \cong R^{q-1} F X^{1} \cong \ldots \cong R^{1} F X^{q-1} \cong \operatorname{Coker}\left(F I^{q-1} \rightarrow F X^{q}\right)$.

Note que para qualquer complexo $C^{\bullet}$, os operadores de bordo $C_{i-1} \rightarrow C_{i}$ podem ser

decompostos da seguinte forma:

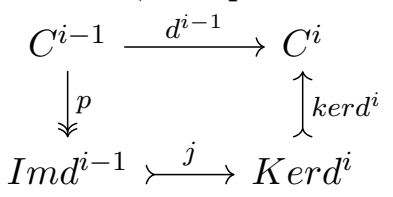

Por definição de cohomologia em categoria abeliana, $H^{q}\left(C^{\bullet}\right)=$ Cokerj. Como $p$ é epi, Coker $\mathrm{C} \cong \operatorname{Coker}(j \circ p)$.

No nosso caso, $C^{\bullet}=F I^{\bullet}$ e a decomposição fica:

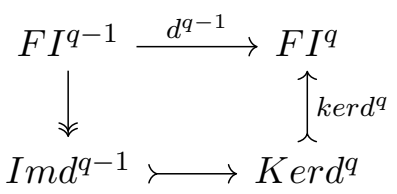

Pela exatidão, $K e r d^{q} \cong F X^{q}$, então o diagrama fica

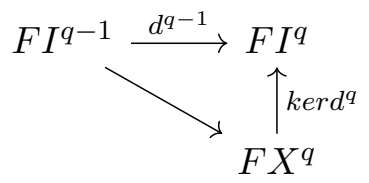

Portanto, $H^{q}\left(F I^{\bullet}\right) \cong \operatorname{Coker}\left(F I^{q-1} \rightarrow F X^{q}\right)$ e, como já vimos, $\operatorname{Coker}\left(F I^{q-1} \rightarrow\right.$ $\left.F X^{q}\right) \cong R^{q} F A$.

Vale resultado análogo para os funtores derivados à esquerda.

Em [Gro57], A. Grothendieck introduz a noção de $\delta$-funtores, é um conceito um pouco mais geral que funtores derivadas que nos permite fornecer uma propriedade universal para eles.

Definição 1.4.14 Sejam $\mathscr{C}$ e $\mathscr{C}^{\prime}$ categorias abelianas. Chamamos de $\delta$-funtor (covariante) entre $\mathscr{C}$ e $\mathscr{C}^{\prime}$ uma coleção de funtores aditivos $T_{\bullet}=\left\{T_{n}: \mathscr{C} \rightarrow \mathscr{C}^{\prime} \mid n \geq 0\right\}$ tal que para toda sequência exata curta $E: 0 \rightarrow A \rightarrow B \rightarrow C \rightarrow 0$ e $n \geq 0$ existem morfismos $\delta_{n}: T_{n}(C) \rightarrow T_{n-1}(A)$ satisfazendo as seguintes condições:

1. Para cada sequência exata curta $E$ temos que a sequência longa

$$
\ldots T_{n+1}(C) \stackrel{\delta}{\longrightarrow} T_{n}(A) \longrightarrow T_{n}(B) \longrightarrow T_{n}(C) \stackrel{\delta}{\longrightarrow} T_{n-1}(A) \ldots
$$

é exata.

2. Para cada morfismo entre sequências exatas curtas $E$ e $E^{\prime}$

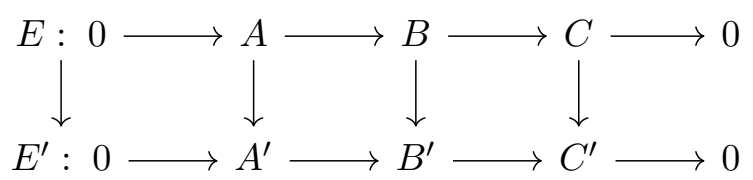

os $\delta$ 's induzem o seguinte diagrama comutativo

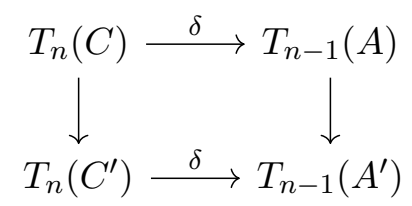


Acima definimos um $\delta$-funtor homológico, para o caso cohomológico adota-se uma notação levemente diferente e os diagramas são adapatados da forma adequada (a sequência "sobe"). Temos, por exemplo, $\delta^{n}: T^{n}(C) \rightarrow T^{n+1}(A)$. Feita essa observação acrescentamos ainda que $T^{n}=T_{n}=0$ para $n<0$, por convenção.

Note que $T_{0}$ é exato à direita e $T^{0}$ é exato à esquerda.

Definição 1.4.15 Dizemos que $\alpha_{\bullet}: S_{\bullet} \rightarrow T_{\bullet}$ é um morfismo de $\delta$-funtores se $\alpha_{\bullet}=$ $\left\{\alpha^{n}: S^{n} \rightarrow T^{n} \mid n \geq 0\right\}$ é uma coleção de transformações naturais e tal que para toda sequência exata curta $E$ o seguinte diagrama comuta

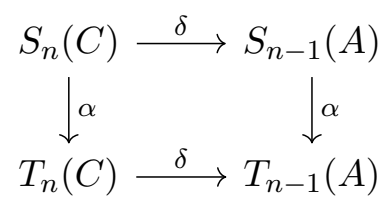

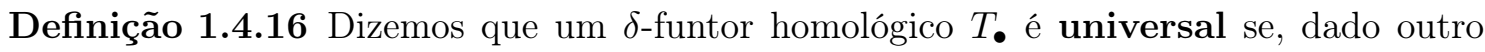
$\delta$-funtor $S_{\bullet}$ e uma transformação natural $\alpha_{0}: S_{0} \rightarrow T_{0}$, existe um único morfismo $\left\{\alpha_{n}\right.$ : $\left.S_{n} \rightarrow T_{n}\right\}$ de $\delta$-funtores que estende $\alpha_{0}$. Analogamente definimos $\delta$-funtor cohomológico $T^{\bullet}$ universal

Apenas pela definição de $\delta$-funtores dá para ver a semelhança com funtores derivados, mas para encerrar essa seção vamos enunciar, sem provar, os resultados que explicitam como $\delta$-funtores são uma versão mais geral de funtores derivados com uma noção de universalidade.

Teorema 1.4.17 Sejam $\mathscr{C}$ e $\mathscr{C}^{\prime}$ categorias abelianas e $T^{\bullet}: \mathscr{C} \rightarrow \mathscr{C}^{\prime}$ um $\delta$-funtor. Se para cada $A$ objeto de $\mathscr{C}$ existe um monomorfismo $m: A \rightarrow B$ tal que $T^{n}(m)=0, \forall n>0$, então $T^{\bullet}$ é universal.

Na Proposição 2.2.1 de [Gro57] é feita a demonstração desse resultado.

Corolário 1.4.18 Sejam $\mathscr{C}$ e $\mathscr{C}^{\prime}$ categorias abelianas onde $\mathscr{C}$ possui suficiente objetos injetivos. Considere $F: \mathscr{C} \rightarrow \mathscr{C}^{\prime}$ exato à esquerda. Então $R^{\bullet} F$ é $\delta$-funtor universal.

\section{Demonstração:}

Como $F$ é exato à esquerda, $R^{q} F$ satisfaz as quatro condições do Teorema 1.4.9, logo, é imediato que forma um $\delta$-funtor. Seja $A$ um objeto de $\mathscr{C}$, considere $m: A \rightarrow I$ um mono em um objeto injetivo $I$. Pela observação $1.4 .12, R^{q} F(I)=H^{q} F\left(I^{\bullet}\right)=0, \forall q>0$, então $R^{q} F(m)=0, \forall q>0$. Pelo Teorema 1.4.17, $R^{\bullet} F$ é $\delta$-funtor universal.

Note que vale uma recíproca do resultado acima, se $T^{\bullet}$ for um $\delta$-funtor universal então $T^{0}$ é exato à esquerda e $T^{q} \cong R^{q} T^{0}$ é isomorfismo. Temos $T^{0}$ é exato à esquerda simplesmente pela definição de $\delta$-funtor. Usando que $\mathscr{C}$ possui suficientes injetivos, garantimos que os funtores derivados $R^{q} T^{0}$ existem, além disso, como acabamos de ver, $R^{q} T^{0}$ são $\delta$-funtores universais. Por definição de universal existe no máximo um funtor $T^{\bullet}$ tal que $R^{0} T^{0} \cong T^{0}$. Já vimos que $R^{0} T^{0}$ existe e esse iso vale pelo Teorema 1.4.9, item (ii). Logo, para cada $q \geq 0$, vale o iso $T^{q} \cong R^{q} T^{0}$.

\subsection{Funtor Ext}

Seremos breves, mas já que falamos de funtores derivados devemos apresentar um dos exemplos mais comuns e famosos: o funtor Ext. Vimos que o funtor $\operatorname{Hom}_{\mathscr{C}}(-, I)$ é exato à esquerda, será que existe alguma forma de estender a sequência de forma a obter a exatidão do lado direito também? Sim, vamos ver isso agora. 
Optamos por ser mais concretos nessa seção, em vez trabalharmos com categorias abelianas vamos considerar $R$-módulos com $R$ anel comutativo com unidade. Estamos seguindo [HS71].

Sejam $A, B, C$ e $I R$-módulos e considere $0 \rightarrow A \rightarrow B \rightarrow C \rightarrow 0$ sequência exata curta. Aplicando o funtor $\operatorname{Hom}(-, I)$, pela Proposição 1.2.3, obtemos uma sequência exata da forma $0 \rightarrow \operatorname{Hom}(C, I) \rightarrow \operatorname{Hom}(B, I) \rightarrow \operatorname{Hom}(A, I)$. Note que

$$
0 \rightarrow \operatorname{Hom}(C, I) \rightarrow \operatorname{Hom}(B, I) \rightarrow \operatorname{Hom}(A, I) \rightarrow \operatorname{Coker}(\operatorname{Hom}(B, I) \rightarrow H o m(A, I)) \rightarrow 0
$$

é exata, tal Coker é um $R$-módulo de cohomologia, logo, por definição de funtor derivado à direita:

$$
\operatorname{Ext}^{1}(A, I)=R^{1} \operatorname{Hom}(A, I)
$$

Então $E x t^{1}$, ou simplesmente $E x t$, é quem resolve a nossa questão, mas podemos ainda definir $\operatorname{Ext}^{q}$ para $q \geq 0$ :

Definição 1.5.1 Seja $I$ um $R$-módulo, definimos $\mathbf{E x t}^{q}$ como o $q$-ésimo funtor derivado à direita de $\operatorname{Hom} R(-, I)$

$$
\left.\operatorname{Ext}^{q}(A, I)=R^{q} \operatorname{Hom}(A, I)\right),
$$

sendo que $\operatorname{Ext}^{0}(A, I)=\operatorname{Hom}(A, I)$.

Assim, por propriedade de funtor derivado à direta, dada $0 \rightarrow A \rightarrow B \rightarrow C \rightarrow 0$ exata obtemos uma sequência exata longa da forma:

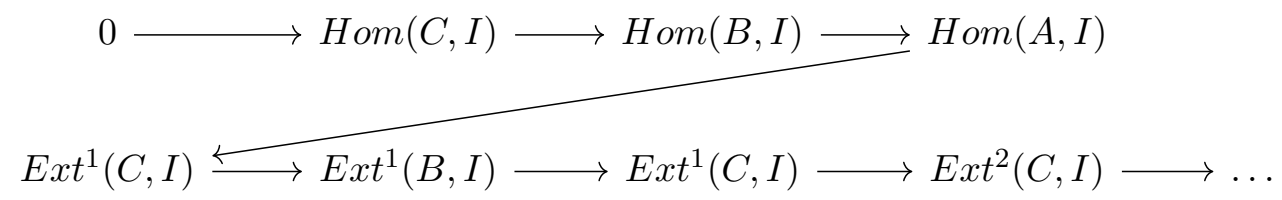

Vamos utilizar essa sequência exata longa para caracterizar objetos injetivos por Ext:

\section{Proposição 1.5.2 São equivalente:}

(i) I é R-módulo injetivo.

(ii) $\operatorname{Hom}(-, I)$ é um funtor exato.

(iii) $\operatorname{Ext}^{q}(A, I)=0, \forall q \geq 0, \forall A \in R$-Mod

(iv) $\operatorname{Ext}^{1}(A, I)=0, \forall A \in R$-Mod

\section{Demonstração:}

Já temos que $(i)$ e $(i i)$ são equivalentes pelo Lema 1.4.2

$($ ii $) \Longrightarrow($ iii $)$ : Se $\operatorname{Hom}_{R}(-, I)$ é exato então é, em particular, exato à direita. Todo funtor exato à direita $F$ satisfaz $R^{q} F(I)=0$, para todo $q>0$. Portato, $\operatorname{Ext}^{q}(-, I)=$ $R^{q} \operatorname{Hom}(-, I)=0$ para todo $q>0$.

$($ iii $) \Longrightarrow($ iv $)$ : É imediato, tomando $q=1$.

$(i v) \Longrightarrow(i i):$ Sabemos que

$$
0 \rightarrow \operatorname{Hom}(C, I) \rightarrow \operatorname{Hom}(B, I) \rightarrow \operatorname{Hom}(A, I) \rightarrow \operatorname{Ext}^{1}(C, I)
$$

é exata. Como $\operatorname{Ext}^{1}(C, I)=0$, obtemos:

$$
0 \rightarrow \operatorname{Hom}(C, I) \rightarrow \operatorname{Hom}(B, I) \rightarrow \operatorname{Hom}(A, I) \rightarrow 0
$$

exata, isto é, o funtor $\operatorname{Hom}(-, I)$ é exato. 
Equivalências análogas caracterizam objetos projetivos e a demonstração é similar. Agora vamos fazer alguns cálculos com o caso mais simples que podemos pensar $\mathbb{Z}$ e $\mathbb{Z}_{r}$, onde $r \in \mathbb{Z}$ com $r \neq 0$. Nesse caso temos $R-M o d \cong A b$.

Como $\mathbb{Z}$ é um grupo abeliano livre, é um grupo projetivo e, pela versão dual da Proposição 1.5 .2 , temos que $\operatorname{Ext}(\mathbb{Z}, \mathbb{Z})=0=\operatorname{Ext}\left(\mathbb{Z}, \mathbb{Z}_{r}\right)$.

Exemplo 1.5.3 $\operatorname{Ext}\left(\mathbb{Z}_{r}, \mathbb{Z}\right)=\mathbb{Z}$

Considere $0 \longrightarrow \mathbb{Z} \succ \stackrel{f}{\longrightarrow} \mathbb{Z} \longrightarrow \mathbb{Z}_{r} \longrightarrow 0$ sequência exata curta onde $f(x)=$ $r x, \forall x \in \mathbb{Z}$

Aplicando o funtor $\operatorname{Hom}(-, \mathbb{Z})$ :

$$
0 \rightarrow \operatorname{Hom}\left(\mathbb{Z}_{r}, \mathbb{Z}\right) \rightarrow \operatorname{Hom}(\mathbb{Z}, \mathbb{Z}) \stackrel{f^{*}}{\rightarrow} \operatorname{Hom}(\mathbb{Z}, \mathbb{Z}) \rightarrow \operatorname{Ext}\left(\mathbb{Z}_{r}, \mathbb{Z}\right) \rightarrow \operatorname{Ext}(\mathbb{Z}, \mathbb{Z})=0
$$

é exata e $f^{*}$ é também multiplicação por $r$. Usando que $\operatorname{Hom}\left(\mathbb{Z}_{r}, \mathbb{Z}\right)=0$, pois $r \neq 0$ e $\operatorname{Hom}(\mathbb{Z}, \mathbb{Z})=\mathbb{Z}$ caímos na sequência exata curta

$$
0 \longrightarrow \mathbb{Z} \stackrel{f}{\longrightarrow} \mathbb{Z} \longrightarrow \operatorname{Ext}\left(\mathbb{Z}_{r}, \mathbb{Z}\right) \longrightarrow 0
$$

Logo,

$$
0 \longrightarrow \mathbb{Z}_{r} \stackrel{\cong}{\operatorname{Ext}}\left(\mathbb{Z}_{r}, \mathbb{Z}\right) \longrightarrow 0
$$

Então $\operatorname{Ext}\left(\mathbb{Z}_{r}, \mathbb{Z}\right)=\mathbb{Z}_{r}$

Já que falamos da categoria $R$-Mod, vamos discutir um pouco a relação entre o caso concreto - $R$-Mod - e o caso abstrato - categorias abelianas de forma geral.

Quando somos introduzidos ao mundo das categorias, temos como primeiros exemplos casos concretos, isto é, sabemos exatamente quem são os objetos e os morfismo. Em $R$-Mod os objetos são $R$-módulos e os morfismos são homomorfismos de $R$-módulos. No entanto existem casos em que isso não ocorre, são o que chamamos de categorias "abstratas", tais como as categorias abelianas. É simplesmente uma categoria satisfazendo certas propriedades, mas não dizemos - em geral - quem são os objetos nem quem são os morfismos, apenas sabemos que obedecem alguns axiomas.

A questão é que não temos acesso direto aos objetos, pois normalmente não são estruturas contretas (isto é, um conjunto munido de operações e relações). Dessa forma, muitos resultados não podem ser demonstrados, entre eles, o já citado Lema da Serpente.

Não importa se estamos querendo provar algo sobre grupos, conjuntos, espaços topológicos, $R$-módulos etc., em Matemática é muito comum começarmos uma demonstração já considerando um elemento do conjunto que estamos estudando, mas sem ter um acesso aos objetos como vamos saber a relação entre os elementos? Uma forma de contornar essa situação é através do Teorema de Freyd-Mitchell, onde garantimos que categorias abelianas (pequenas) podem ser mergulhadas na categoria dos $R$-Mod.

Teorema 1.5.4 (Mergulho de Freyd-Mitchell) Toda categoria abeliana pequena admite um funtor exato, pleno e fiel para a categoria dos $R$-Mod, onde $R$ é algum anel com unidade.

\section{Demonstração:}

Teorema 7.34 de [Fre64]

No livro de P. Freyd o enunciado do Teorema está diferente, mas o que ele chama de "fully abelian" significa dizer que a categoria abeliana admite funtor exato, pleno e fiel para a categoria dos $R$-Mod. 
Não entramos nos detalhes desse Teorema e suas implicações, pois preferimos atacar essa questão do acesso aos objetos de uma forma que vale para qualquer categoria abeliana: os pseudoelementos, introduzidos nas seção 1.7. Contudo, é possível encontrar uma aplicação de 1.5.4 para demonstrar o Lema da Serpente em [Wei95] (Lema 1.3.2).

\subsection{Sequências Espectrais}

Sequências Espectrais são uma técnica para calcular objetos de (co)homologia, no entanto, seremos breves nessa seção e apresentaremos apenas o que será utilizado quando formos trabalhar com cohomologia em topos.

Almejando essa agilidade na apresentação do tema usamos a apresentação de [Gro57], mas com algumas modificações para termos uma abordagem mais moderna, para isso usamos [Wei95]. Recomendamos a leitura de [HS71] e [McC01] para uma profundidade maior no tema.

Nessa seção voltamos a usar $\mathscr{C}$ para categoria abeliana.

Definição 1.6.1 Seja $A$ objeto de $\mathscr{C}$. Uma filtração (decrescente) em $A$ é uma família $\left\{F^{p}(A) \mid p \in \mathbb{Z}\right\}$ de subojetos de $A$ tal que

$$
0 \subseteq \ldots \subseteq F^{p+1}(A) \subseteq F^{p}(A) \subseteq \ldots \subseteq A
$$

Se $A$ e $B$ são objetos de $\mathscr{C}$ equipados com filtrações, se um morfismo $u: A \rightarrow B$ é tal que $u\left(F^{q}(A)\right) \subseteq F^{q}(B)$, para todo $q \in \mathbb{Z}$, dizemos que $u$ é compatível com as filtrações.

Definição 1.6.2 Uma sequência espectral (cohomológica) que começa em $E_{a}$ é uma dupla $\left(E_{r}^{p, q}, E_{a}\right)$ onde:

1. Uma família de objetos $\left\{E_{r}^{p, q}\right\}$ de $\mathscr{C}$ com inteiros $p, q, r \geq a$.

2. Morfismos $d_{r}^{p, q}: E_{r}^{p, q} \rightarrow E_{r}^{p+r, q-r+1}$ chamados diferencias tais que $d_{r}^{p+r, q-r+1} \circ d_{r}^{p, q}=0$.

3. Isomorfismos $E_{r+1}^{p, q} \cong H^{p, q}\left(E_{r}\right)=\operatorname{Ker}\left(d_{r}^{p, q}\right) / \operatorname{Im}\left(d_{r}^{p-r, q+r-1}\right)$.

Chamaremos os objetos $E_{r}^{p, q}$ de termos, mas é bastante comum na literatura usarem página ou folha.

Note que os complexos de cocadeia são um exemplo de sequência espectral cohomológica onde $a=0=q, r=1$, os termos $E_{r}^{p}$ são os objetos $C_{r}^{p}$ munidos com operadores de bordo $d_{C}^{p}: C^{p} \rightarrow C^{p+1}$.

Definição 1.6.3 O número $n=p+q$ é dito grau total do termo $E_{r}^{p, q}$.

Definição 1.6.4 Uma sequência espectral (cohomológica) é dita limitada se existe um número finito de termos não-nulos em cada grau total.

Se isso ocorre, então para cada $p$ e $q$ existe $r_{0}$ tal que $d_{r}^{p, q}=0$ para todo $r \geq r_{0}$, logo, a sequência $E_{r}^{p-r, q+r-1} \rightarrow E_{r}^{p, q} \rightarrow E_{r}^{p+r, q-r+1}$ se torna nula e temos que $E_{r+1}^{p, q} \cong$ $\operatorname{Ker}\left(d_{r}^{p, q}\right) / \operatorname{Im}\left(d_{r}^{p-r, q+r-1}\right) \cong \operatorname{Ker}\left(d_{r}^{p, q}\right) \cong E_{r}^{p, q}$, para todo $r \geq r_{0}$. Escrevemos $E_{\infty}^{p, q}$ para esse valor estável.

Definição 1.6.5 Uma sequência espectral limitada converge para $H^{n}, n=p+q$, se existe uma filtração (decrescente) finita tal que

$$
0=F^{n+1} H^{n} \subseteq \ldots \subseteq F^{p+1} H^{n} \subseteq F^{p} H^{n} \subseteq \ldots \subseteq F^{0} H^{n}=H^{n}, n=p+q
$$

e temos isomorfismos $E_{\infty}^{p, q} \cong F^{p} H^{n} / F^{p+1} H^{n}$. 
Notação: $E_{r}^{p, q} \Longrightarrow H^{n}$, onde $r$ é o grau de limitação.

Teorema 1.6.6 (Sequência Espectral de Grothendieck) Sejam $\mathscr{A}, \mathscr{B}$ e $\mathscr{C}$ categorias abelianas tais que $\mathscr{A}$ e $\mathscr{B}$ possuem suficientes injetivos e $S: \mathscr{A} \rightarrow \mathscr{B}$ e $T: \mathscr{B} \rightarrow \mathscr{C}$ são dois funtores exatos à esquerda. Se $S$ envia objetos injetivos de $\mathscr{A}$ em objetos $T$-acíclicos de $\mathscr{B}$, então existe uma sequência espectral $E_{r}^{p, q}$ de funtores $\mathscr{A} \rightarrow \mathscr{C}$ tal que $E_{r}^{p, q} \cong\left(R^{p} T\right)\left(R^{q} S\right)$ e é convergente para $E_{\infty}^{p, q} \cong R^{p+q}(T \circ S)$, isto é, $E_{r}^{p, q} \Longrightarrow R^{p+q}(T \circ S)$.

\section{Demonstração:}

Teorema 9.3 de [HS71]

A sequência da conclusão desse Teorema é a sequência espectral de $T \circ S$.

Apesar de não apresentarmos a demonstração desse resultado, ele será utilizado diversas vezes ao longo do capítulo de Álgebra Homológica em Topos.

Vamos trabalhar pelo menos um pouco com sequências espectrais, mostrando um resultado que será utilizado futuramente.

Seja $E_{2}^{p, q} \Longrightarrow H^{n}$ e suponha $E_{2}^{p, q}=0$ para todo $p$ ou $q$ negativos (esse sempre será nosso caso, mas poderíamos ter $E_{2}^{p, q}$ diferente de zero mesmo nessas condições).

Pela definição de sequência espectral temos que $E_{r+1}^{p, q} \cong \operatorname{Ker}\left(d_{r}^{p, q}\right) / \operatorname{Im}\left(d_{r}^{p-r, q+r-1}\right)$, então, fixando $p=0, E_{r+1}^{0, q} \cong \operatorname{Ker}\left(d_{r}^{0, q}\right) / \operatorname{Im}\left(d_{r}^{-r, q+r-1}\right)$. Logo, $E_{r+1}^{0, q} \cong \operatorname{Ker}\left(d_{r}^{0, q}\right)$, pois $d_{r}^{-r, q+r-1}: E_{r}^{-r, q+r-1}=0 \rightarrow E_{r}^{0, q}$. Uma vez que o kernel é um mono, isso nos fornece a seguinte sequência de monomorfismos:

$$
E_{\infty}^{0, q} \longmapsto \ldots \longmapsto E_{r}^{0, q} \longmapsto E_{r-1}^{0, q} \longmapsto \ldots \longmapsto E_{2}^{0, q}
$$

Analogamente, se variarmos $p>0$ e fixarmos $q=0$ obtemos uma sequência de epimorfismos

$$
E_{2}^{p, 0} \rightarrow \ldots \rightarrow E_{r-1}^{p, 0} \rightarrow E_{r}^{p, 0} \rightarrow \ldots \rightarrow E_{\infty}^{p, 0}
$$

Agora usamos $E_{\infty}^{p, q} \cong F^{p} H^{n} / F^{p+1} H^{n}$, onde $n=p+q$. Então temos as sequências exatas

$$
\begin{gathered}
F^{1} H^{p+q} \longrightarrow F^{0} H^{p+q} \cong H^{p+q} \longrightarrow E_{\infty}^{0, p+q} \\
\vdots \\
F^{p+q} H^{p+q} \longrightarrow F^{p+q-1} H^{p+q} \longrightarrow E_{\infty}^{p+q-1,1} \\
0=F^{p+q+1} H^{p+q} \longrightarrow F^{p+q} H^{p+q} \longrightarrow E_{\infty}^{p+q, 0}
\end{gathered}
$$

Pegue a primeira sequência e faça $p=0$, então

$$
F^{1} H^{q} \longrightarrow F^{0} H^{q} \cong H^{q} \longrightarrow E_{\infty}^{0, q}
$$

Então $E_{\infty}^{0, q}$ é quociente de $H^{q}$, unindo esse fato à sequência de monomorfismos, obtemos

$$
H^{q} \rightarrow E_{\infty}^{0, q} \longmapsto E_{2}^{0, q}
$$

Observando as sequências exatas também concluímos que $E_{\infty}^{p, 0} \cong F^{p} H^{p}$, logo, $E_{\infty}^{p, 0}$ é um subobjeto de $H^{p}$. Unindo com a sequência de epimorfismos obtemos:

$$
E_{2}^{p, 0} \rightarrow E_{\infty}^{p, 0} \longmapsto H^{p}
$$

Assim, acabamos de mostrar: 
Proposição 1.6.7 Se $E_{2}^{p, q}$ é uma sequência espectral que converge para $H^{n}$ e $E_{2}^{p, q}=0$ se $p<0$ ou $q<0$, então temos

$$
H^{q} \rightarrow E_{\infty}^{0, q} \longmapsto E_{2}^{0, q}
$$

$e$

$$
E_{2}^{p, 0} \rightarrow E_{\infty}^{p, 0} \nrightarrow H^{p}
$$

\subsection{Complementos sobre Categorias Abelianas}

Reservamos essa última seção para destrincharmos o problema levantado logo no início do capítulo sobre não termos elementos em objetos de categorias abelianas e também para trazer a hierarquia de axiomas que categorias abelianas podem ou não ter, os conhecidos ABn's introduzidos pela primeira vez em [Gro57].

No que diz respeito à chamada caça em diagramas, diferentemente do que ocorre para a categoria dos módulos sobre um anel, nem sempre uma categoria abeliana se comporta como a categoria $A b$ ou $R$-Mod, isto é, nem sempre temos um conjunto subjacente (objeto da categoria) onde os elementos desse conjunto se comportam da forma esperada. Vamos contornar isso através dos pseudoelementos, como feito em [Bor94b].

Definição 1.7.1 Considere um objeto $A$ e um morfismo $f: A \rightarrow B$, ambos em uma categoria abeliana $\mathscr{C}$ :

1. Denominamos por pseudoelemento de $A$ um morfismo a com contradomínio $A$, ou seja, $\bullet \stackrel{a}{\rightarrow} A$. Notação: $a \in^{*} A$.

2. Dois pseudoelementos $X \stackrel{a}{\rightarrow} A$ e $X^{\prime} \stackrel{a^{\prime}}{\rightarrow} A$ são pseudoiguais quando existem epimorfismos $Y \stackrel{p}{\rightarrow} X$ e $Y \stackrel{p^{\prime}}{\longrightarrow} X^{\prime}$ tais que $a \circ p=a^{\prime} \circ p^{\prime}$. Notação: $a={ }^{*} a^{\prime}$

3. A pseudoimagem sobre $f: A \rightarrow B$ de $a \in^{*} A$ é a composta $f \circ a$. Notação: $f(a)$.

Note que se dois pseudoelementos são pseudoiguais, então suas respectivas pseudoimagens também são, isto é, sejam $a \in^{*} A$ e $a^{\prime} \in^{*} A$ são tais que $a=^{*} a^{\prime}$, então $f(a)={ }^{*} f\left(a^{\prime}\right)$. Também é fácil ver que vale $f(g(a))={ }^{*}(f \circ g)(a)$. Observamos ainda, sem demonstrar, que a noção de pseudoigualdade define uma relação de equivalência nos pseudoelementos de $A .{ }^{4}$ e que devido a fatoração em epi e mono, os pseudo-elementos de $A$ correspondem aos subobjetos de $A$.

Agora enunciamos um lema que servirá para auxiliar na demonstração da proposição que o sucede.

Lema 1.7.2 Seja A um objeto de uma categoria abeliana. Existe uma classe de equivalência para pseudoigualdades em pseudoelementos de A que consiste exatamente de todos os zeros morfismos com codominio em A.

\section{Demonstração:}

O que faremos é mostrar que dado um pseudoelemento $a \in^{*} A$, temos $a=^{*} 0 \Longleftrightarrow$ $a=0$, onde 0 é visto como um pseudo elemento de $A$.

Começamos supondo $a: X \rightarrow A$ pseudoigual a $0: Y \rightarrow A$. Por definição, existem epimorfismo $p$ e $q$ tais que $a \circ p=0 \circ q$ mas $0 \circ q=0=0 \circ p$, $\log$ o, $a=0$, pois $p$ é um epi.

Por outro lado, supondo $a=0$, tomamos como epimorfismo as projeções $p_{X}$ e $p_{Y}$ (projeções são retrações e por isso são epis) e obtemos $a \circ p_{X}=0=0 \circ p_{Y}, \operatorname{logo}, a={ }^{*} 0$.

Proposição 1.7.3 Seja $\mathscr{C}$ uma categoria abeliana. Então:

(i) $f: A \rightarrow B$ é morfismo nulo $\Longleftrightarrow f(a)={ }^{*} 0, \forall a \in^{*} A$.

\footnotetext{
${ }^{4}$ Isto depende do fato de que, em categorias abelianas, pullbacks de epis são epis
} 
(ii) $f: A \rightarrow B$ é monomorfimo $\Longleftrightarrow\left(\forall a, a^{\prime} \in{ }^{*} A, f(a)={ }^{*} f\left(a^{\prime}\right) \Rightarrow a={ }^{*} a^{\prime}\right)$.

$\Longleftrightarrow\left(\forall a \in^{*} A, f(a)={ }^{*} 0 \Rightarrow a={ }^{*} 0\right)$

(iii) $f: A \rightarrow B$ é epimorfismo $\Longleftrightarrow \forall b \in^{*} B \exists a \in^{*} A$ tal que $f(a)={ }^{*} b$.

(iv) $A \stackrel{f}{\rightarrow} B \stackrel{g}{\rightarrow} C$ é uma sequência exata $\Longleftrightarrow \forall a \in^{*} A g(f(a))={ }^{*} 0 e$ $\forall b \in^{*} B, g(b)={ }^{*} 0 \Rightarrow \exists a \in^{*}$ A tal que $f(a)={ }^{*} b$.

(v) $f: A \rightarrow B$ e a, $a^{\prime} \in{ }^{*} A, f(a)={ }^{*} f\left(a^{\prime}\right) \Rightarrow\left(\exists a^{\prime \prime} \in^{*}\right.$ A tal que $f\left(a^{\prime \prime}\right)={ }^{*} 0 e \forall g: A \rightarrow C$, $\left.g\left(a^{\prime}\right)={ }^{*} 0 \Rightarrow g\left(a^{\prime \prime}\right)={ }^{*} g(a)\right)$.

\section{Demonstração:}

(i) $(\Longrightarrow)$ Temos $f=0$, logo, $f(a)=0$. Pelo Lema 1.7.2, $f(a)={ }^{*} 0$

$(\Longleftarrow)$ Como $f(a)=^{*} 0, \forall a \in^{*} A$, em particular, $f\left(i d_{A}\right)={ }^{*} 0$. Usando novamente o Lema 1.7.2 obtemos, $f=0$.

(ii) Primeiro suponha $f$ mono. Seja $f(a)={ }^{*} f\left(a^{\prime}\right), \forall a, a^{\prime} \in \in^{*} A$. Então existem epimorfismos $p$ e $q$ tais que $f \circ a \circ p=f \circ a^{\prime} \circ q$. Como $f$ é mono, $a \circ p=a^{\prime} \circ q$, logo, $a^{\prime}={ }^{*} a$.

Note que se $a^{\prime}=0$, então $f\left(a^{\prime}\right)=0 \Longrightarrow a={ }^{*} 0$. Assim fizemos as "idas". Para fechar as equivalências veja que

$$
f \circ(a)=0 \Longrightarrow f(a)={ }^{*} 0 \Longrightarrow a={ }^{*} 0 \Longrightarrow a=0 \text {, pelo Lema 1.7.2. }
$$

Pelo Lema 1.1.18, $f$ é mono.

(iii) $(\Longrightarrow)$ Tome $b \in^{*} B$, podemos construir o pullback de $f$ e $b$ :

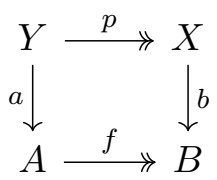

onde $a \in^{*} A$ e $p$ é epi pois $f$ é epi. Dessa forma, $f \circ a \circ i d_{Y}=b \circ p$ e, portanto, $f(a)={ }^{*} b$. Caso não esteja claro, usamos $i d_{Y}$ pois é um epimorfismo que nos permite concluir a pseudoigualdade.

$(\Longleftarrow)$ Tomamos $i d_{B} \in^{*} B$ ea $\in^{*} A$ e epimorfismos $p, q$ tais que $f \circ a \circ p=i d_{B} \circ q=q$. Então $f \circ a \circ p$ é epi, logo, $f$ é epi.

(iv) $(\Longrightarrow)$ Seja $A \stackrel{f}{\rightarrow} B \stackrel{g}{\rightarrow} C$ uma sequência exata, então $g \circ f=0$. Pelo item $(i)$ que provamos a pouco, $g(f(a))={ }^{*} 0$.

Agora considere $b \in^{*} B$ tal que $g(b)={ }^{*} 0$ e uma fatoração em epi e mono $f=i \circ p$, $\log i=\operatorname{Im} f$.

Assim $A \stackrel{p}{\longrightarrow} I \stackrel{i}{\longrightarrow} B \stackrel{g}{\longrightarrow} C$ e $i=\operatorname{Imf}=$ kerg.

Usando o item $(i)$ mais uma vez, temos que $g \circ b=0$ e podemos considerar uma fatoração $b=i \circ c$ e agora temos:

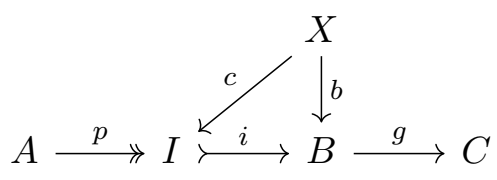

Calculando o pullback de $p$ e $c$ obtemos: 


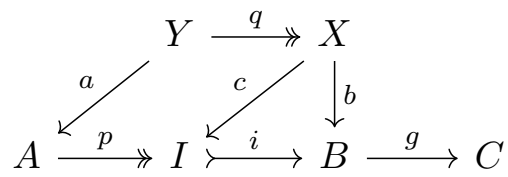

Então $f \circ a \circ i d_{Y}=i \circ p \circ a=b \circ q$ e concluímos $f(a)={ }^{*} b$, pois $q$ é epi.

$(\Longleftarrow)$ Consideramos $f=i$ op uma fatoração em epi e mono, mostraremos que $i=k e r g$ e isso encerra a prova, pois $i=\operatorname{Imf}$

Por hipótese $g(f(a))={ }^{*} 0, \forall a \in \in^{*} A$, então, pelo item $(i), g \circ i \circ p=g \circ f=0$. Como $p$ é epi, $g \circ i=0$. Já temos que $i$ anula $g$, logo, $\operatorname{Im} f \leq k e r g$.

Vamos provar que kerg $\leq \operatorname{Imf}$.

Por hipótese, $g(b)={ }^{*} 0$ também implica que existe $a \in^{*} A$ e epimorfismos $q, r$ tais que $i \circ p \circ a \circ r=b \circ q$.

Calculando o pullback de $i$ e $b$ e já considerando o que faremos a seguir, temos o diagrama

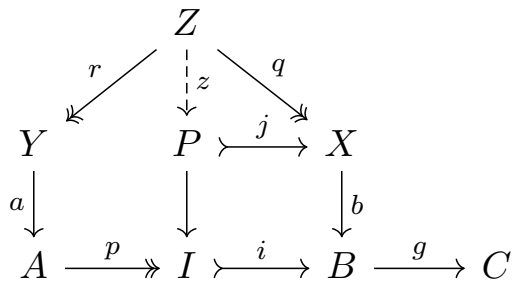

O diagrama, além do pullback está nos dando uma fatoração $q=j \circ z$ e $c \circ z=p \circ a \circ r$.

Como $i$ é mono, $j$ também é. Como $q$ é epi, $j \circ z$ também é, logo, $j$ é.

Portanto, $j$ é um iso e podemos fazer $b=i \circ c \circ j^{-1}$. Essa fatoração é única pois $i$ é mono. Em particular, tomando $b=\operatorname{kerg}: \operatorname{Kerg} \longmapsto B$, vemos que $[b] \leq[i]$, logo, $k e r \leq \operatorname{Imf}$.

(v) Temos pseudoelementos $a: X \rightarrow A$ e $a^{\prime}: X^{\prime} \rightarrow A$ e epis $p: Y \rightarrow X, p^{\prime}: Y \rightarrow X^{\prime}$ tais que $f \circ a \circ p=f \circ a^{\prime} \circ p^{\prime}$.

Vamos chamar $a^{\prime \prime}=a \circ p-a^{\prime} \circ p^{\prime}$. Assim:

$$
f\left(a^{\prime \prime}\right)=f\left(a \circ p-a^{\prime} \circ p^{\prime}\right)=f \circ a \circ p-f \circ a^{\prime} \circ p^{\prime}=0 \Longrightarrow f\left(a^{\prime \prime}\right)={ }^{*} 0
$$

Se $g\left(a^{\prime}\right)={ }^{*} 0$, então existem epis $q: Z \rightarrow X$ e $q^{\prime}: Z \rightarrow X^{\prime}$ tais que $g \circ a^{\prime} \circ q^{\prime}=0 \circ q$. Considere o pullback abaixo:

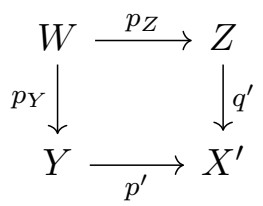

Daí $g \circ \circ a^{\prime} \circ q^{\prime} \circ p_{Z}={ }^{*} 0 \mathrm{e}$

$$
g\left(a^{\prime \prime}\right)={ }^{*} g \circ\left(a^{\prime \prime} \circ p_{Y}\right)={ }^{*} g \circ a \circ p \circ p_{Y}-g \circ \circ a^{\prime} \circ q^{\prime} \circ p_{Z}={ }^{*} g \circ a \circ p \circ p_{Y} \Longrightarrow g\left(a^{\prime \prime}\right)={ }^{*} g(a)
$$

A proposição acima é de fundamental importância para a demonstração dos grandes lemas de caça aos diagramas, como veremos a seguir para o caso do Lema da Serpente. Para tal demonstração iremo lanças mão do seguinte resultado: 
Lema 1.7.4 Considere o seguinte retângulo comutativo em uma categoria abeliana, com epis e monos conforme indicado

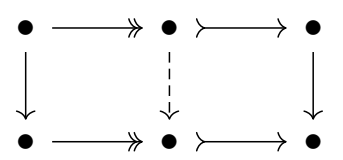

Então existe um único morfismo $h$ que torna ambos os quadrados comutativos

\section{Demonstração:}

Proposição 1.5.6 de [Bor94b]

Lema 1.7.5 (Lema da Serpente) Considere o seguinte diagrama em uma categoria abeliana

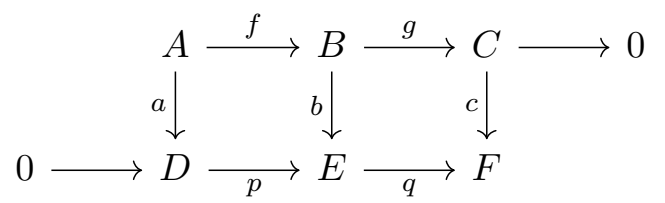

onde $A \rightarrow B \rightarrow C \rightarrow 0$ e $0 \rightarrow D \rightarrow E \rightarrow F$ são exatas e os quadrados comutam. Então o diagrama abaixo comuta

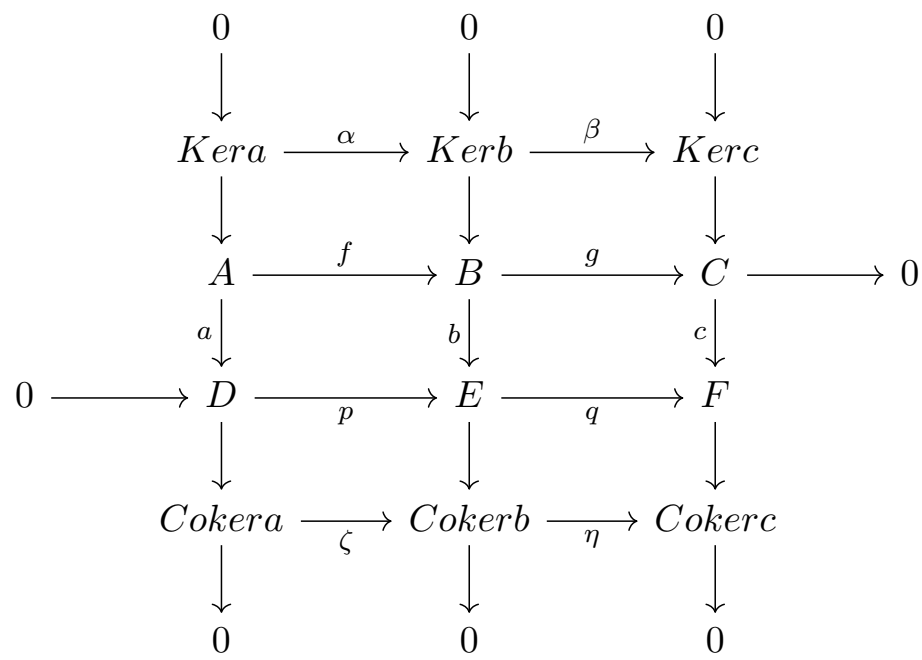

e existe morfismo $\partial:$ Kerc $\rightarrow$ Cokera tal que a sequência $0 \rightarrow$ Kera $\rightarrow$ Kerb $\rightarrow$ Kerc $\rightarrow$ Cokera $\rightarrow$ Cokerb $\rightarrow$ Cokerc $\rightarrow 0$ é exata.

\section{Demonstração:}

Já avisamos que essa é uma demonstração bem longa e trabalhosa, seremos mais cuidadosos nas primeiras etapas, mas em certo ponto iremos usar livremente propriedades do kernel, do cokernal e a Proposição 1.7.3 sem mencioná-las explicitamente.

Começamos mostrando que a sequência Kera $\stackrel{\alpha}{\rightarrow}$ Kerb $\stackrel{\beta}{\rightarrow}$ Kerc é exata, utilizando a equivalência (iii) da Proposição 1.7.3.

Como $A \stackrel{f}{\rightarrow} B \stackrel{g}{\rightarrow} C \rightarrow 0$ é exata, temos $g \circ f=0$, logo

$$
\operatorname{kerc} \circ \beta \circ \alpha=g \circ f \circ \operatorname{ker} a=0 \circ \operatorname{ker} a=\operatorname{kerc} \circ 0
$$

Como kerc é mono, temos $\beta \circ \alpha=0$, pelo Lema 1.7.2, $\beta(\alpha(x))={ }^{*} 0$ para todo $x \in \epsilon^{*}$ Kera.

Agora tome $x \in^{*}$ Kerb tal que $\beta(x)={ }^{*} 0$. Veremos que existe $z \in^{*}$ Kera tal que $\alpha(z)={ }^{*} x$. Temos

$$
g(k e r b \circ x)=(g \circ \operatorname{kerb})(x)={ }^{*}(\operatorname{kerc} \circ \beta)(x)={ }^{*}(\operatorname{kerc})(0)={ }^{*} 0
$$


Como $A \stackrel{f}{\rightarrow} B \stackrel{g}{\rightarrow} C \rightarrow 0$ é exata, pela Proposição 1.7.3, existe $y \in^{*} A$ tal que $f(y)={ }^{*}$ $(k e r b)(x)$.

Veja que $(p \circ a)(y)={ }^{*}(b \circ f)(y)={ }^{*}(b \circ k e r b)(x)={ }^{*} 0$. Como $p$ é mono, pela Proposição 1.7.3 (ii), segue que $a(y)={ }^{*} 0$.

Usado que $0 \rightarrow D \stackrel{p}{\rightarrow} E \stackrel{q}{\rightarrow} F$ é exata, existe $z \in^{*}$ Kera tal que $k e r a(z)={ }^{*} y$. Então $(\operatorname{kerb} \circ \alpha)(z)={ }^{*} f \circ \operatorname{kera}(z)={ }^{*} f(y)={ }^{*}(k e r b)(x)$.

Como kerb é mono, concluímos $\alpha(z)={ }^{*} x$ como queríamos.

Analogamente, verifica-se que Cokera $\stackrel{\zeta}{\rightarrow}$ Coerb $\stackrel{\eta}{\rightarrow}$ Cokerc é exata

Agora consideramos decomposições $f=f_{2} \circ f_{1}$ e $q=q_{2} \circ q_{1}$ e aplicamos o Lema 1.7.4 duas vezes, então existem únicos morfismos $\gamma$ e $\lambda$ que fazem cada um dos quadrados do diagrama abaixo comutarem.

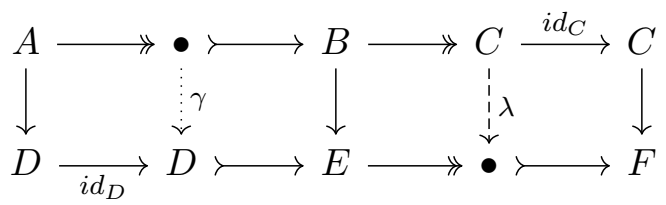

Podemos considerar os Kerneis e Cokerneis de $\gamma$ e $\lambda$ de forma que obtemos o seguinte diagrama comutativo, o qual garante a comutatividade desejada para esse Lema (para mais detalhes ver o Lema 1.10.8 de [Bor94b])

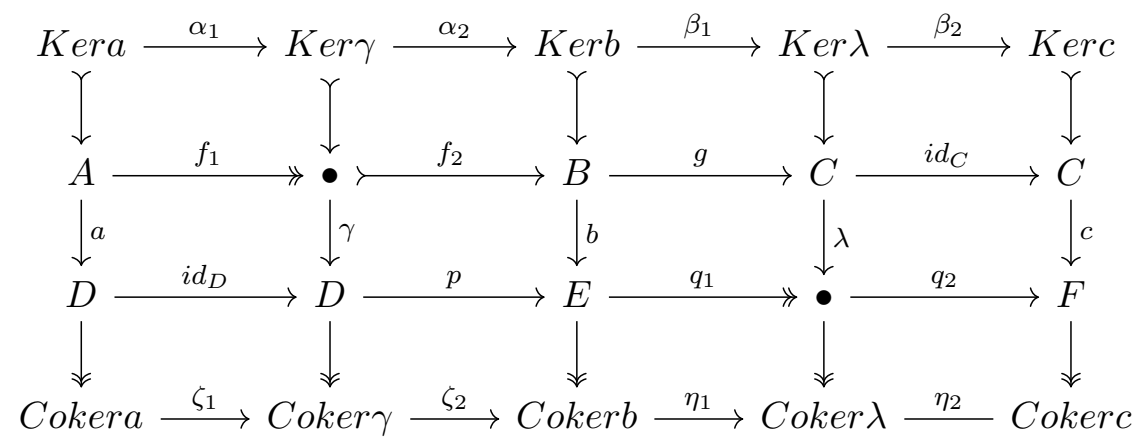

Estamos considerando $\alpha=\alpha_{2} \circ \alpha_{1}, \beta=\beta_{2} \circ \beta_{1}, \zeta=\zeta_{2} \circ \zeta_{1}, \eta=\eta_{2} \circ \eta_{1}$. Desta forma, cada uma das colunas é exata.

Como cada um dos quadrados comuta, temos $\alpha_{2}$ e $\beta_{2}$ monos e $\zeta_{1}$ e $\eta_{1}$ epis.

Agora queremos mostrar que $\beta_{2}$ é um isomorfismo. Pela Proposição 1.1.13, basta mostrar que também é um epi.

Seja $w \in \in^{*}$ Kerc, temos $\left(q_{2} \circ \lambda \circ \operatorname{kerc}\right)(w)={ }^{*}(c \circ k e r c)(w)={ }^{*} 0$. Como $q_{2}$ é mono, $\lambda \circ \operatorname{kercc}={ }^{*} 0$. Pela exatidão da coluna considerada, existe $u \in \operatorname{Ker} \lambda$ tal que $(\operatorname{ker} \lambda)(u)={ }^{*}$ $(\operatorname{kerc})(w)$. De ker $\circ \beta_{2}=\operatorname{ker} \lambda$ segue que $\left(\operatorname{ker} \circ \beta_{2}\right)(u)={ }^{*}(\operatorname{kerc})(w), \log \circ, \beta_{2}(u)={ }^{*} w$, pois kerc é mono. Portanto, $\beta_{2}$ é epi (Proposição 1.7.3).

Dualmente, verifica-se que $\zeta_{1}$ é isomorfismo.

Note que a exatidão da sequência $A \stackrel{f}{\rightarrow} B \stackrel{g}{\rightarrow} C$ implica na exatidão de $\bullet \stackrel{f_{2}}{\rightarrow} B \stackrel{g}{\rightarrow} C$. Analogamente, a exatidão de $D \stackrel{p}{\rightarrow} E \stackrel{q}{\rightarrow} P$ implica na exatidão de $D \stackrel{p}{\rightarrow} E \stackrel{q_{1}}{\rightarrow} \bullet$. Usaremos isso para mostrar que existe $\chi$ tal que Kerb $\stackrel{\beta_{1}}{\longrightarrow} \operatorname{Ker} \lambda \stackrel{\chi}{\rightarrow}$ Coker $\gamma \stackrel{\zeta_{1}}{\longrightarrow}$ Cokerb é uma sequência exata.

Via propriedades universais, construiremos um morfismo $\chi: \operatorname{Ker} \lambda \rightarrow$ Coker $\gamma$. Se fizermos o pullback de $k e r \lambda$ e $g$, obteremos morfismos $\Lambda$ e $\Sigma$. Se fizermos o pushout de ker $\gamma$ e $p$ obteremos morfismos $\Gamma$ e $\Pi$. Tomamos o kernel de $\Sigma$ e o cokernel de $\Pi$ (note que $\Pi$ é mono pois pushout de mono é mono), assim existem morfismos $\varphi$, $\psi$ tais que o seguinte diagrama comuta e as colunas da esquerda e da direta são exatas (para mais detalhes ver o Lema 1.10.8 de [Bor94b]). 


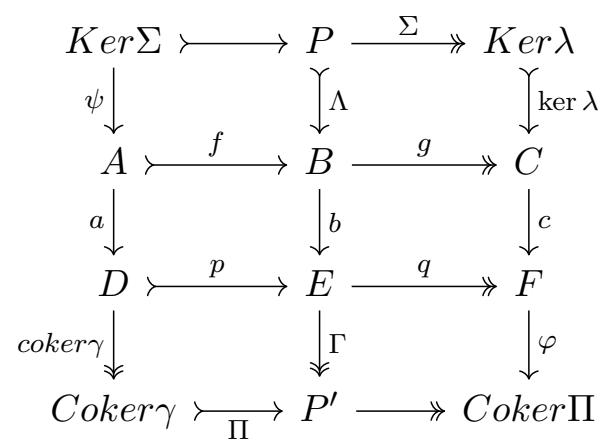

Assim temos $\Gamma \circ b \circ \Lambda \circ \operatorname{ker} \Sigma=\Pi \circ \operatorname{coker} \gamma a \circ \psi=0$, pela exatidão da coluna esquerda.

Mas note que pelo Teorema 1.1.17 (fatoração em epi e mono), como $\Sigma$ é epi, temos $\Sigma=\operatorname{coker}(\operatorname{ker} \Sigma)$. Analogamente temos $\Pi=\operatorname{ker}(\operatorname{coker} \Pi)$. Então existe um único $\omega$ tal que $\omega \circ \Sigma=\Gamma \circ b \circ \Lambda$ tal que $(\operatorname{coker} \Pi)(\omega)=0$ - pois $\Sigma$ é epi.

Ainda, $(\operatorname{coker} \Pi)(\omega)=0$ e $\Pi=\operatorname{ker}(\operatorname{coker} \Pi)$ implicam que existe único $\chi$ tal que

$$
\Pi \circ \chi=\omega
$$

Considere então o morfismo $\chi: \operatorname{Ker} \lambda \rightarrow$ Coker $\gamma$ conforme contruímos acima, a partir de agora faremos uso do método dos pseudo-elementos.

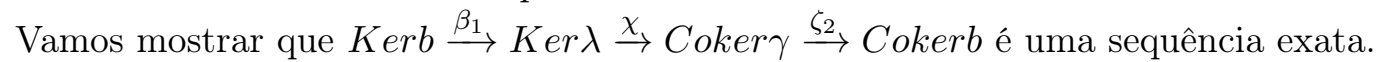

Começamos verificando que dado $x \in^{*} \operatorname{Kerb},\left(\chi \circ \beta_{1}\right)(x)=0$.

Temos $g \circ(k e r b) \circ x={ }^{*} \operatorname{ker} \lambda \circ \beta_{1} \circ x, \log \circ$,

$$
(q \circ b) \circ(k e r b) \circ x={ }^{*}(\lambda \circ g) \circ(k e r b) \circ x={ }^{*} \lambda \circ \operatorname{ker} \lambda \circ \beta_{1} \circ x=0
$$

Assim, $b \circ(k e r b) \circ x \in^{*} \operatorname{Kerq}_{1}=\operatorname{Kerq}=\operatorname{Imf}$ e $b \circ(k e r b) \circ x={ }^{*} p(k)$ para algum $k \in \in^{*} D$. Como $p$ é mono, obtemos $k={ }^{*} 0$.

Por outro lado, usando a definição de pseudoigualdade, $g \circ($ kerb $) \circ x={ }^{*} k e r \lambda \circ \beta_{1} \circ x$ implica que existem epis $e$ e $e^{\prime}$ tais que $g \circ(k e r b) \circ x \circ e=k e r \lambda \circ \beta_{1} \circ x \circ e^{\prime}$. Por definição de pullback existe $v \in^{*} P$ tal que $\Sigma(v)={ }^{*} \beta_{1}(x)$ e $\Lambda(v)={ }^{*}(k e r b) \circ x$.

Como vale $(\star)$ :

$$
\begin{aligned}
(\Pi \circ \chi)\left(\beta_{1}(x)\right)={ }^{*} \omega\left(\beta_{1}(x)\right)={ }^{*} \omega(\Sigma(v)) & ={ }^{*}(\Gamma \circ b \circ \Lambda)(v)={ }^{*}(\Gamma \circ b) \circ(k e r b) x \\
& ={ }^{*} \Gamma \circ p \circ k={ }^{*} \Pi \circ(\text { coker } \gamma) \circ k
\end{aligned}
$$

Como $\Pi$ é mono, concluímos $\left(\chi \circ \beta_{1}\right)(x)={ }^{*}$ coker $\gamma \circ k={ }^{*} 0$, pois $k={ }^{*} 0$

Agora considere $l \in^{*} \operatorname{Ker} \lambda, m \in^{*} B$ e $k \in^{*} D$. Note que se substituirmos $\beta_{1}(x)$ por $l$ e $(k e r b) \circ x$ por $m$ nas contas que fizemos acima, ainda teremos que $p(k)={ }^{*} b(m)$ e $g(m)=(k e r \lambda) \circ l$.

Suponha $($ coker $\gamma) \circ k={ }^{*} 0$, então existe $d$ tal que $\gamma(d)={ }^{*} k$. Veja que $(b \circ f)(d)={ }^{*}(p \circ$ $f)(d)={ }^{*} p(k)={ }^{*} b(m)$, como $m$ e $f(d)$ são pseudo elementos de $B$ temos $b(m-f(d))={ }^{*} 0$.

De $(g \circ f)(d)={ }^{*} 0$ segue que $g(m-f(d))={ }^{*} g(m)$, mas $b(m-f(d))={ }^{*} 0$ implica que existe $x \in^{*} B$ tal que $(k e r b) \circ x=^{*}(m-f(d))$.

Vale ainda

$$
(\text { ker } \lambda) \circ \beta_{1} \circ x={ }^{*} g \circ k e r b \circ x={ }^{*} g(m-f(d))={ }^{*} g(m)={ }^{*}(k e r \lambda) \circ l
$$

Como ker $\lambda$ é mono, concluímos que $\beta_{1} \circ x={ }^{*} l$ e, portanto, Kerb $\stackrel{\beta_{1}}{\rightarrow} \operatorname{Ker} \lambda \stackrel{\chi}{\rightarrow}$ Coker $\gamma$ é sequência exata.

Agora considere novamente $l \in^{*} \operatorname{Ker} \gamma, m \in^{*} B$ e $k \in^{*} D$ nas mesmas condições de antes. Então:

$$
\zeta_{2} \circ \chi(l)={ }^{*} \zeta_{2} \circ(\operatorname{coker} \gamma)(k)={ }^{*}(\operatorname{cokerb} \circ p)(k)={ }^{*}(\operatorname{cokerb} \circ b)(m)={ }^{*} 0
$$


Seja $j \in \epsilon^{*}$ Coker $\gamma$ tal que $\zeta_{2}(j)={ }^{*} 0$. Como coker $\gamma$ é epimorfismo, existe $k \in^{*} D$ tal que $(\operatorname{coker} \gamma)(k)={ }^{*} j$. Mas $0={ }^{*}(\operatorname{cokerb} \circ p)(m)={ }^{*} \zeta_{2} \circ(\operatorname{coker} \gamma)(k)={ }^{*} \zeta_{2}(j)$, logo, obtemos $m \in^{*} B$ tal que $b(m)={ }^{*} p(k)$.

Ainda, $(\lambda \circ g)(m)=^{*}\left(q_{1} \circ b\right)(m)={ }^{*}\left(q_{1} \circ p\right)(k)={ }^{*} 0$. Então temos $l \in \in^{*} k e r \lambda$ tal que $\operatorname{ker} \lambda(l)={ }^{*} g(m)$. Pela descrição de $\chi$, encontramos $j={ }^{*}($ coker $\gamma)(k)={ }^{*} \chi(l)$. Portanto $\operatorname{Ker} \lambda \stackrel{\chi}{\rightarrow}$ Coker $\gamma \stackrel{\zeta_{2}}{\longrightarrow}$ Cokerb é uma sequência exata.

Enfim concluímos que Kerb $\stackrel{\beta_{1}}{\longrightarrow} \operatorname{Ker} \lambda \stackrel{\chi}{\rightarrow}$ Coker $\gamma \stackrel{\zeta_{1}}{\longrightarrow}$ Cokerb é sequência exata.

Para finalizarmos a demonstração basta definir $\partial=\zeta_{1}^{-1} \circ \chi \circ \beta_{2}^{-1}$. Como $\zeta_{1}$ e $\beta_{2}$ são isomorfismos, a sequência $\operatorname{Kerb} \stackrel{\beta}{\rightarrow} \operatorname{Kerc} \stackrel{\partial}{\rightarrow}$ Cokera $\stackrel{\zeta}{\rightarrow}$ Cokerb é exata. Uma vez que já mostramos que as sequências Kera $\rightarrow$ Kerb $\rightarrow$ Kerc e Cokera $\rightarrow$ Cokerb $\rightarrow$ Cokerc, é imediato que

$$
0 \rightarrow \text { Kera } \rightarrow \text { Kerb } \rightarrow \text { Kerc } \rightarrow \text { Cokera } \rightarrow \text { Coker } b \rightarrow \text { Cokerc } \rightarrow 0
$$

é exata.

Com essa demonstração temos a consciência limpa de que o trabalho realizado ao longo desse capítulo de fato vale. Outro lemas de caça em diagramas necessários na Álgebra Homológica também podem ser demonstrados para categorias abelianas utilizando esse mesmo recurso dos pseudoelementos.

Vamos agora comentar um pouco sobre os $A B n^{\prime} s$. As propriedades 1 e 2 da definição 1.1.12 são mais conhecidas como $A B 1$ e $A B 2$, respectivamente. Os $A B n^{\prime} s$ basicamente existem como axiomas que conferem às categorias abelianas propriedades a mais, quanto maior o $n$, mais forte é o axioma, vamos listá-los. Seja $\mathscr{C}$ uma categoria abeliana:

$A B 3)$ Seja $\left\{A_{i}\right\}_{i \in I}$ família de objetos de $\mathscr{C}$, então a soma direta de $A_{i}^{\prime}$ existe.

$A B 4)$ Vale $A B 3$ e que a soma direta de uma família de monomorfismo é também um monomorfismo.

$A B 5)$ Vale $A B 3$ e se $\left\{A_{i}\right\}_{i \in I}$ é uma família dirigida de subobjetos de um objeto $A$ de $\mathscr{C}$ e $B$ é qualquer outro subobjeto de $A$, então $\left(\sum_{i \in I} A_{i}\right) \cap B=\sum_{i \in I}\left(A_{i} \cap B\right)$, onde $\sum A_{i}$ denota o sup de $A_{i}$ e a intersecção denota o inf dos subobjetos.

Há ainda o axioma $A B 6$, mas iremos parar por aqui.

Adiante precisaremos de $A B 5$ para garantir que podemos trabalhar com cohomologia em um topos, mas nosso interesse não se restringe a esse ponto específico. Ao longo desse trabalho surgiu uma questão interessante sobre formas de representar a categoria dos grupos abelianos em um topos através de categorias abelianas com algumas propriedades a mais, é uma questão, até onde sabemos, sem resposta sobre a qual teceremos mais comentários no último capítulo desse trabalho, pensando em pesquisas futuras. Por hora, essas observações devem ser suficientes.

Alguns exemplos de categorias $A B 5$ são:

- $R$-Módulos;

- Feixes de $R$-Módulos;

- Feixes de grupos abelianos munidos com uma topologia de Grothendieck (provaremos isso no capítulo 3);

- Dado $G$ um grafo orientado e $\mathscr{C} A B 5$, a categoria dos diagramas $G \rightarrow \mathscr{C}$. 


\section{Capítulo 2}

\section{Teoria de Topos}

Neste capítulo tratamos de outro tema ingrediente dessa Dissertação, o qual será relacionado com a Álgebra Homológica, tema desenvolvido no capítulo anterior, ao longo do Capítulo 3.

Enquanto o estudo dos feixes de grupos abelianos levaram às categorias abelianas, o estudo dos feixes de conjuntos levaram aos topos de Grothendieck, os quais rapidamente foram generalizados na forma de topos elementares.

Quando F.W. Lawvere entrou em contato com o trabalho de A. Grothendieck sobre topos ele acreditou que poderia axiomatizar a física clássica através de uma fundamentação de geometria diferencial (atualmente essa fundamentação é conhecida como geometria diferencial sintética), contudo, ele buscou uma forma de tornar a complexa estrutura dos topos de Grothendieck mais simples, mantendo o que fosse essencial. Em um trabalho conjunto com M. Tierney, axiomas categoriais bastante concisos deram origem ao conceito de topos elementar.

Hoje em dia a Teoria de Topos possui as mais diversas aplicações e pode ser vista de várias perspectivas. Na introdução de [Joh02a] P. Johnstone lista 13 formas de descrever um topos, mais do que justificando o título "Sketches of an Elephant" - topos remetem à parábola dos cegos que podiam tocar um elefante, cada um sentia uma parte do elefante: um a cauda, outro a tromba, um terceiro as patas e assim por diante. Separadamente, pode parecer que se trata de um animal totalmente diferente, mas a verdade é que todas compõem o elefante. Nós, ao longo da Dissertação abordamos os aspectos geométrico (como um tipo de feixe) e o lógico (através de observações sobre linguagem e as álgebras de Heyting).

Aproveitamos essa introdução para desfazer um senso comum: não é verdade que os topos surgiram como uma tentativa de generalizar a categoria dos conjuntos. De fato, a Sets é um exemplo de topos e topos seria, de certa forma, a categoria que mais se aproxima de Sets (qualquer topos pode ser obtido através de equivalência de categorias como uma categoria de conjuntos com uma teoria de conjuntos local, veja [Bel08]), mas historicamente surgem do trabalhos de A. Grothendieck em Álgebra Homológica e Geometria Algébrica, o interesse de F.W. Lawvere nos fundamentos da Física e de M. Tierney em Topologia Algébrica. Eventualmente se referir ao topos como uma generalazição de conjuntos pode ser útil para tornar o assunto mais próximo da maioria dos matemáticos, afinal, nossa formação é conjuntista. Mais detalhes sobre como essa confusão ocorreu e um pouco da história da teoria de topos se encontram no artigo [Mc190].

Nesse capítulo começamos definindo feixes de conjuntos e indicamos a equivalência entre a categoria de feixes e a categoria dos espaços étale como feito em [MLM94] e em [Flo13]. Na seção seguinte, usando a mesma referência, explicamos como as topologia de Grothendieck surgem para definir a noção de feixes em categorias que não possuem uma noção de aberto como os espaços topológicos e com isso conseguimos introduzir os Topos de Grothendieck. Na terceira e quarta seção usamos também [Joh14] para definir os topos elementares e introduzimos os morfismos parciais representáveis principalmente porque 
historicamente eles tiveram um papel importante - já fizeram parte da própria definição de topos, mas logo perceberam que eram uma consequência. Posteriormente falamos dos morfismos geométricos, são um dos morfismos interessantes entre topos, e é fundamental dessa parte em diante ter certa familiaridade com adjunção. Recomendamos [Bor94a] caso não conheça. As duas últimas seções mostram topos como um objeto de particular interesse da Lógica, nelas [Bor94c] foi bastante utilizado.

\section{$2.1 \quad$ Feixes de Conjuntos}

Nessa seção providenciaremos uma definição categorial de feixes, isto é, como um certo funtor contravariante, mas também iremos relacionar com a perspectiva geométrica indicando o passo a passo para ver que todo feixe de conjunto pode ser representado como feixe de seções do fibrado étale. Essas duas visões, que estamos chamando aqui de categorial e geométrica, estão tão conectadas que fornecem uma equivalência de categorias. A principal referência dessa seção [MLM94]

Antes do formalismo, vamos tentar criar um pouco de intuição. Imagine um espaço qualquer e para cada aberto nesse espaço, considere uma família de funções definidas neste aberto, satisfazendo uma certa propriedade. Suponha que se você tomar outros abertos nesse mesmo espaço, eles também satisfazem essa propriedade. Além disso, funções definidas nesses aberto coincidem nos pontos de intersecção para cada par de abertos. Um feixe nos dirá que a propriedade dessas funções vale no espaço todo. Definimos uma (única) função para esse espaço todo que coincide com cada uma das outras funções se considerarmos as restrições para seus respectivos abertos - informação local revela informação global.

Quando falarmos do fibrado étale veremos que o nome feixe realmente faz sentido. É como se colocássemos cada uma dessas funções lado a lado e amarrássemos elas todas juntas por uma propriedade de colagem, dada pela topologia do espaço em que estivermos.

Se nossa tentativa de fornecer uma intuição tiver funcionado, deve ser possível dar o palpite que o conjunto das funções contínuas em um aberto é um exemplo de feixe. Vamos começar a definir e depois voltamos para esse exemplo.

Definição 2.1.1 Seja $X$ um espaço topológico. Um pré-feixe $\mathscr{F}$ em $X$ é uma aplicação que para cada aberto $U$ de $X$ associa um conjunto $\mathscr{F}(U)$ e para cada inclusão entre abertos $U \subseteq V$ tem associado o mapa restrição $\operatorname{res}_{U}^{V}: \mathscr{F}(V) \rightarrow \mathscr{F}(U)$ de forma que as seguinte condições são satisfeitas:

1. Para todo aberto $U$ de $X, \operatorname{res}_{U}^{U}=i d_{\mathscr{F}(U)}$

2. Se temos a inclusão de abertos $U \subseteq V \subseteq W$, então $\operatorname{res}_{U}^{W}=r e s_{U}^{V} \circ \operatorname{res} s_{V}^{W}$.

É claro que um pré-feixe em um espaço topológico $X$ é simplemente um funtor $\mathscr{F}$ : $\mathcal{O}(X)^{o p} \rightarrow$ Sets da categoria oposta dos conjuntos parcialmente ordenados dos abertos de $X$ na categoria Sets. Dessa forma um morfismo de pré-feixes é uma transformação natural.

É possível trocar Sets por outras categorias, faremos isso mais adiante para $A b$, a categoria dos grupos abelianos.

Chamamos os elementos de $\mathscr{F}(U)$ de seções de $\mathscr{F}$ sobre $U$.

Notação: É usual e adotaremos ao longo do texto escrever apenas $s_{\left.\right|_{U}}$ em vez de $\operatorname{res}_{U}^{V}(s)$, se $U \subseteq V$ e $s \in \mathscr{F}(V)$.

Definição 2.1.2 O pré-feixe $\mathscr{F}$ é dito feixe se para todo aberto $U$ em $X$ e toda cobertura aberta $U=\bigcup_{i \in I} U_{i}$ valem as seguintes condições:

1. (Propriedade da colagem) Dado $s_{i} \in \mathscr{F}\left(U_{i}\right)$ tal que $s_{i_{U_{i} \cap U_{j}}}=s_{j_{U_{i} \cap U_{j}}}$ para todo $i, j \in I$. Então existe um $s \in \mathscr{F}(U)$ tal que $s_{\left.\right|_{i}}=s_{i}$.

2. (Unicidade da colagem) Seja $s, s^{\prime} \in \mathscr{F}(U) \operatorname{com} s_{\left.\right|_{U_{i}}}=s_{\left.\right|_{U_{i}}}^{\prime}$. Então $s=s^{\prime}$ 
Quando o pré-feixe satisfaz apenas a condição 2 acima, dizemos que ele é separável.

Denotamos a categoria dos feixes sobre um espaço topológico $X$ por $S h(X)$, onde os objetos são os feixes e os morfismos são os morfismos de feixes - que são os morfismos de pré-feixes.

Vamos tornar ao exemplo do conjunto das funções contínuas. Sejam $X$ e $Y$ espaços topológicos e considere o pré-feixe $C^{Y}$ das funções contínuas $f$ de $X$ em $Y$ de forma que para cada aberto $U$ em $X$ temos $C^{Y}(U)$, o conjunto de funções contínuas do tipo $U \rightarrow Y$, onde para cada par de abertos $U \subseteq V$ temos $s_{U}^{V}: C^{Y}(V) \rightarrow C^{Y}(U), f \mapsto f_{\left.\right|_{U}}$. Considere uma cobertura aberta $U_{i}$ de $U$ e funções contínuas $f_{i}: U_{i} \rightarrow Y$. Sabemos que para funções contínuas se $f_{i}(x)=f_{j}(x)$ para todo $x \in U_{i} \cap U_{j}$, existe exatamente uma função $f: U \rightarrow X$ tal que $f_{\left.\right|_{i}}=f_{i}$. Além disso, como as $f_{i}$ são contínuas e os $U_{i}$ são abertos, então $f$ também é contínua. Assim, $C^{Y}$ é um feixe.

De forma similar, os pré-feixes de funções diferenciáveis e analíticas são feixes. Para maiores detalhes e mais exemplos ver a referência ([Ten75])

Também podemos descrever um feixe de conjuntos em termos de um diagrama de equalizador.

Definição 2.1.3 O pré-feixe $\mathscr{F}$ é dito feixe se os abertos $U$ em $X$ tais que $U=\bigcup_{i \in I} U_{i}$ determinam um equalizador

$$
\mathscr{F}(U) \stackrel{e}{\longrightarrow} \prod_{i \in I} \mathscr{F}\left(U_{i}\right) \stackrel{p}{\underset{q}{\longrightarrow}} \prod_{(i, j) \in I \times I} \mathscr{F}\left(U_{i} \cap U_{j}\right)
$$

onde

1. $e(t)=\left\{t_{\left.\right|_{U_{i}}} \mid i \in I\right\}, \quad t \in \mathscr{F}(U)$

2. $p\left(\left(t_{k}\right)_{k \in I}\right)=\left(t_{i_{U_{i} \cap U_{j}}}\right)_{(i, j) \in I \times I}$ e $q\left(\left(t_{k}\right)_{k \in I}\right)=\left(t_{j_{U_{i} \cap U_{j}}}\right)_{(i, j) \in I \times I}, \quad t_{i} \in \prod_{i \in I} \mathscr{F}\left(U_{i}\right)$

A equivalência entre as definições 2.1.2 e 2.1.3 é bastante imediata uma vez que estamos assumindo certa familiaridade com teoria de categorias.

Agora vamos introduzir os conceitos necessários para falarmos dos feixes geométricos, é interessante como a intuição geométrica dos mesmo justifica a nomenclatura que surge ao longo da teoria de feixes.

Definição 2.1.4 Sejam $X$ e $Y$ espaços topológicos. Um fibrado é um mapa contínuo $p: X \rightarrow Y$. Ainda, para cada $y \in Y$ chamamos de fibra de $X$ sobre $Y$ o subconjunto $X_{y}=p^{-1}(y)=\{x \in X \mid p(x)=y\}$ de $X$.

Podemos pensar nos fibrados como uma família de fibras $x_{y}$, indexadas por $y \in Y$, "coladas" pela topologia de $X$.

Definição 2.1.5 Seja $X$ um espaço topológico. Um fibrado $p_{E}: E \rightarrow X$ é dito étale se $p_{E}$ é um homeomorfismo local, isto é, para cada $x \in E$ existe um conjunto aberto $V$ com $x \in V \subseteq E$ tal que $p(V)$ é aberto em $X$ e $p_{\left.\right|_{V}}: V \rightarrow p(V)$ é um homeomorfismo.

Veja que a projeção $\mathbb{R} \times\{0\} \cup \mathbb{R} \times\{1\} \rightarrow \mathbb{R},(x, i) \mapsto x$ é um fibrado étale.

Outro exemplo é que se fizermos $X=\mathbb{R}^{2}$, os abertos básicos de $X$ são discos abertos, então, para ser fibrado étale, para cada ponto de cada fibra, os abertos $V$ de $E$ são homeomorfos ao disco em $\mathbb{R}^{2}$ (de tamanhos diferentes) e forma-se uma pilha de aberto $V$ ao longo da fibra. 


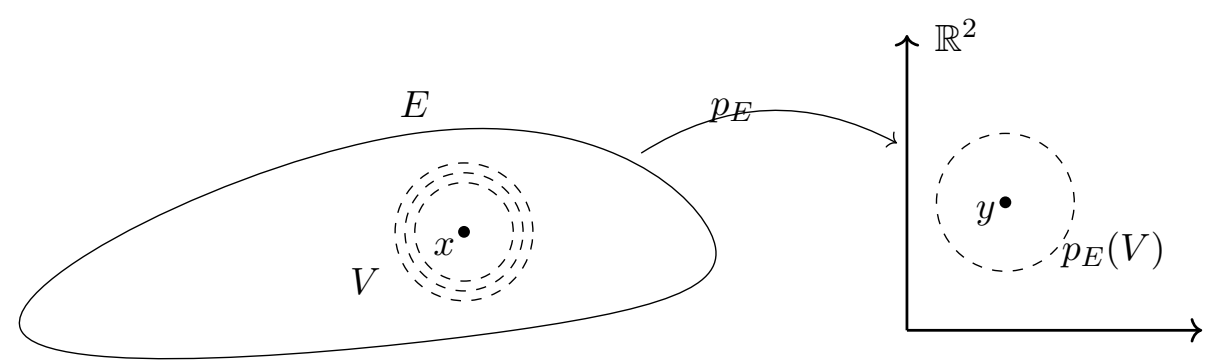

Vamos caminhar para definir uma topologia para os fibrados étale.

Definição 2.1.6 Sejam $p_{E}: E \rightarrow X$ um fibrado e $U \subseteq X$ um subconjunto aberto. A seção de $p_{E}$ em $U$ é um mapa contínuo $s: U \rightarrow E$ tal que $p(s(x))=x, \forall x \in U$.

Infelizmente usamos "seção" tanto para os fibrados como para elementos do pré-feixe aplicados em um aberto $U$, a distinção entre cada um é feita de acordo com o contexto.

Note que $p_{E}$ e sua respectiva seção $s$ são mapas abertos e que, caso $p_{E}$ seja étale, para cada ponto $e \in E$ existe pelo menos um aberto $U$ de $X$ tal que $s: U \rightarrow E$ é seção, isto é, seções locais existem. Assim:

Fato 2.1.7 1. Se $p_{E}: E \rightarrow x$ é um fibrado étale, então uma base da topologia de $E$ é formada pelas imagens $s(U)$ de todas as seções definidas em abertos de $X$.

2. Dadas duas seções $s$ e $t$, o conjunto $W=\{x \mid s(x)=t(x)\}$ é um aberto de $X$. Assim as fibras possuem topologia discreta.

A rigor deveríamos provar que as imagens das seções de fato defininem uma base topológica e que $W$ é aberto, não faremos isso aqui mas deixamos como referência o Lema 3.5 e parte da demonstração da Proposição 3.6 de [Ten75].

Nesse contexto geométrico temos a seguinte noção de feixe:

Definição 2.1.8 Um feixe (geométrico) é um par $\left(E, p_{E}\right)$ tal que $E$ é um espaço topológico e $p_{E}: E \rightarrow X$ é um fibrado étale.

Mas qual é a relação entre essa noção de feixe e a apresentada na Definição 2.1.3? Veremos que todo feixe é um feixe de seções para algum fibrado étale. Para fazer isso precisamos falar de germes.

Definição 2.1.9 Seja $X$ um espaço topológico. Considere o pré-feixe $\mathscr{F}: \mathcal{O}(X)^{o p} \rightarrow$ Sets. Tome $x \in X$, vizinhanças abertas $U$ e $V$ de $x$ e elementos $s \in \mathscr{F}(U)$ e $t \in \mathscr{F}(V)$. Dizemos que $s$ e $t$ possuem o mesmo germe em $x$ se existe algum aberto $W \subseteq U \cap V$ com $x \in W$ e tal que $s_{\left.\right|_{W}}=t_{\left.\right|_{W}} \in \mathscr{F}(W)$.

Note que essa definição é uma relação de equivalência.

Definição 2.1.10 A classe de equivalência de qualquer $s$ como o descrito acima é dito germe de $s$ num ponto $x$. Notação: germe $_{x} s$

Assim, $t \in$ germ $_{x} s$ se, e só se, dados $U, V$ vizinhanças abertas de $x$ existe $W \in U \cap V$ tal que $x \in W$ e $t_{\left.\right|_{W}}=s_{\left.\right|_{W}}$. Ainda, a relação de equivalência dado por "possuir o mesmo germe"nos possibilita a seguinte definição:

Definição 2.1.11 Para cada $x \in X$ definimos a coleção de classes de equivalência, chamado stalk de $\mathscr{F}$ em $x$, por

$$
\mathscr{F}_{x}=\left\{\text { germe }_{x} s \mid s \in \mathscr{F}(U), U \text { vinhança aberta de } x\right\}
$$

Veja que $\mathscr{F}_{x}$ é um colimite:

Como germe $e_{x}^{U} s=$ germe $_{x}^{W} s$ sempre que $x \in W \subseteq U$, temos que germe $x: \mathscr{F}(U) \rightarrow \mathscr{F}_{x}$ forma um cone do tipo 


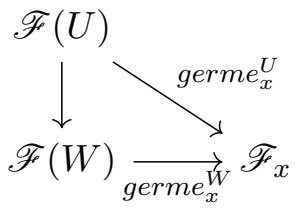

Suponha que existe outro cone $\left\{r^{U}: \mathscr{F}(U) \rightarrow G\right\}_{x \in U}$. Então

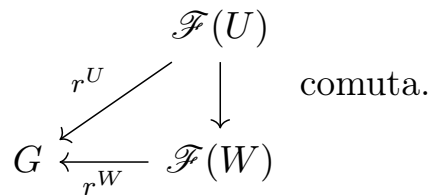

A definição de possuir o mesmo germe implica que existe um único morfismo $t: \mathscr{F}_{x} \rightarrow G$ tal que $t \circ$ germe $e_{x}^{U}=r^{U}$, para todo $U$ vizinhança aberta de $x$. Logo, o seguinte diagrama é comutativo:

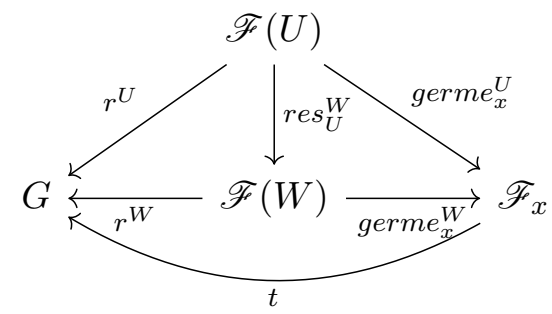

Isso nos diz que germe $e_{x}$ é o cone colimite da restrição do funtor $\mathscr{F}$ por vizinhanças abertas $U$ de $x$. Em outras palavras, $\mathscr{F}_{x}=\underset{x \in U}{\lim _{\longrightarrow}} \mathscr{F}(U)$

Agora vamos começar a construção que nos permite relacionar as duas definições de feixe.

Combine os diversos $\mathscr{F}_{x}$ por uma união disjunta $\Lambda_{\mathscr{F}}=\coprod_{x \in X} \mathscr{F}_{x}$, o conjunto de todos os germes de seções.

Defina uma mapa $p: \Lambda_{\mathscr{F}} \rightarrow X, p\left(\right.$ germe $\left._{x} s, x\right)=x$ e outro mapa $\dot{s}: U \rightarrow \Lambda_{\mathscr{F}}$ tal que $\dot{s}(x)=\left(\right.$ germe $\left._{x} s, x\right)$, para cada $s \in \mathscr{F}(U)$. É claro que $\dot{s}$ é seção de $p$.

Para cada $s$ associamos um único $\dot{s}$, logo, podemos substituir cada $s$ do pré-feixe original $\mathscr{F}(U)$ por uma $\dot{s}$ no conjunto dos germes $\Lambda_{\mathscr{F}}$.

A base da topologia de $\Lambda_{\mathscr{F}}$ é formada pelos conjuntos $\dot{s}(U)$ (e de fato iso determina uma base de topologia em $\left.\Lambda_{\mathscr{F}}\right)$.

Proposição 2.1.12 Com essa construção temos:

(i) $\dot{s}$ é injetora;

(ii) ṡ é mapa aberto;

(iii) $p$ é contínua;

(iv) s̀ é contínua.

\section{Demonstração:}

(i) Se $\left(\right.$ germe $\left._{x}, x\right) s=\left(\right.$ germe $\left._{y} s, y\right)$ temos que $x=y$ pois germes são definidos para um ponto fixado.

(ii) Por definição da base de $\Lambda_{\mathscr{F}}, \dot{s}(U)$ é aberto para todo aberto $U$ e se $V \subseteq U$ é um aberto, $\dot{s}_{\left.\right|_{V}}(x)=\dot{s}(x)$ para todo $x \in V$. Logo, $\dot{s}_{\left.\right|_{V}}=\dot{s}(V)$ e assim temos que $\dot{s}$ é uma palicação aberta.

(iii) Seja $U$ um aberto qualquer de $X$, temos que $p^{-1}(U)=\bigcup_{s_{i} \in \mathscr{F}(U)} \dot{s}_{i}(U)$. Como mapas $\dot{s}_{i}$ são aplicações abertas (pelo item (ii)), cada $\dot{s}_{i}(U)$ é aberto e união de abertos é aberto, logo, $p^{-1}(U)$ também é e, portanto, $p$ é contínua. 
(iv) Seja $x \in U$ e $\dot{t}(V)$ uma vizinhança aberta básica de $\dot{s}(x)$, logo, existe $y \in U$ onde $\left(\right.$ germe $\left._{y}^{V} t, y\right)=\left(\right.$ germe $\left._{x}^{U} s, x\right)$ implica $y=x$. Então existe aberto $W \subseteq U \cap V$ aberto $\operatorname{com} x \in W$ e $s_{\left.\right|_{W}}=t_{\left.\right|_{W}}$.

Assim temos $x \in W \subseteq U$ e abertos $\dot{s}(W)=\dot{t}(W) \subseteq \dot{t}(V)$. Portanto, $\dot{s}$ é contínua.

Corolário 2.1.13 Para todo aberto $U$ e todo $\dot{s} \in \mathscr{F}(U)$ temos que $\dot{s}: U \rightarrow \dot{s}(U)$ é homeomorfismo e $p: \Lambda_{\mathscr{F}} \rightarrow X$ é homeomorfismo local.

\section{Demonstração:}

Pela proposição acima concluímos imediatamente que $\dot{s}: U \rightarrow \dot{s}(U)$ é homeomorfismo. Para ver que $p$ é homeomorfismo local veja que cada germe $e_{x} \in \Lambda_{\mathscr{F}}$ possui vizinhança aberta $\dot{s}(U)$ tal que $p_{\left.\right|_{\dot{s}(U)}}: \dot{s}(U) \rightarrow U$ possui inversa dos dois lados:

$$
p_{\left.\right|_{\dot{s}(U)}} \circ \dot{s}=i d_{U} \text { e } \dot{s} \circ p_{\left.\right|_{\dot{s}(U)}}=i d_{\dot{s}(U)}
$$

Então $p$ é homeomorfismo local.

Resumindo: contruímos um fibrado étale $p$ a partir de um pré-feixe $\mathscr{F}$.

Agora veremos que a partir de um fibrado qualquer podemos contruir um feixe no sentido da Definição 2.1.2.

Teorema 2.1.14 Para qualquer fibrado $p: Y \rightarrow X$ temos que $\Gamma_{p}(U)=\{s: U \rightarrow Y \mid s$ é seção de $p\}$ define um feixe. Esse feixe é dito feixe de seções do fibrado $p$.

\section{Demonstração:}

Com $\Gamma_{p}(U)$ definido dessa forma, dada uma inclusão entre abertos $V \subseteq U$, temos morfismos restrição $\operatorname{res}_{V}^{U}: \Gamma_{p}(U) \rightarrow \Gamma_{p}(V)$ onde facilmente se verifica que $\Gamma_{p}(-): \mathcal{O}(X)^{o p} \rightarrow$ Sets é um funtor, isto é, um pré-feixe.

Vamos verificar que é um feixe.

Seja $U=\bigcup_{i \in I} U_{i}$, começando pela propriedade de colagem, tome $s_{i} \in \mathscr{F}\left(U_{i}\right)$, então $p\left(s_{i}(x)\right)=x$, para todo $x \in U_{i}$.

É claro que existe um morfismo $s: U \rightarrow Y$ onde $s(x)=s_{i}(x)$, para todo $x \in U_{i}$ e tal que $p\left(s_{i}(x)\right)=x$, para todo $x \in U$. Além disso, $s$ é contínua pois $(p \circ s)_{\left.\right|_{U}}=i d_{U}$ e $p$ é uma aplicação aberta (pois é um fibrado). Então $s$ pertence a $\mathscr{F}(U)$.

Para unicidade veja que se tomarmos $s, s^{\prime}$ em $\mathscr{F}(U)$ de forma que $s \neq s^{\prime}$, então $s(x) \neq$ $s^{\prime}(x)$ para algum $x \in U, \operatorname{logo}, s$ e $s^{\prime}$ diferem em esse ponto $x \in U_{i}$ para algum $i \in I$, isso implica que existe $i \in I$ onde $s_{\left.\right|_{U_{i}}} \neq s_{\left.\right|_{U_{i}}}^{\prime}$ e isso é uma contradição. Assim concluímos que $\Gamma_{p}$ é um feixe.

Em particular, temos que $\Gamma_{p}\left(\Lambda_{\mathscr{F}}\right)$ é feixe de seção do fibrado étale $p: \Lambda_{\mathscr{F}} \rightarrow X$. Assim, para cada aberto $U \subseteq X$ existe $\eta_{U}: \mathscr{F}(U) \rightarrow \Gamma\left(\Lambda_{\mathscr{F}}\right)(U), \eta_{U}(s)=\dot{s}$. Note que dado uma aberto $V$ de $U, s_{\left.\right|_{V}}$ coincide com os $\eta$, pois $\left(\dot{s}_{\left.\right|_{V}}\right)(y)=\operatorname{germe} y_{y}^{V}\left(s_{\left.\right|_{V}}\right)=\operatorname{germe}_{y}^{U}(s)=\dot{s}(y)$, para todo $y \in V$. Então $\eta: \mathscr{F} \rightarrow \Gamma\left(\Lambda_{\mathscr{F}}\right)$ é uma tranformação natural.

A partir do fibrado obtivemos um feixe de seções, mas e se esse fibrado fosse obtido de um pré-feixe? O que aconteceria se aquele pré-feixe fosse um feixe? Nesse caso teríamos que $\eta$ é um isomorfismo. Isso será visto no próximo Teorema, mas vamos fazer alguns comentários sobre como visualizar os fibrados étale.

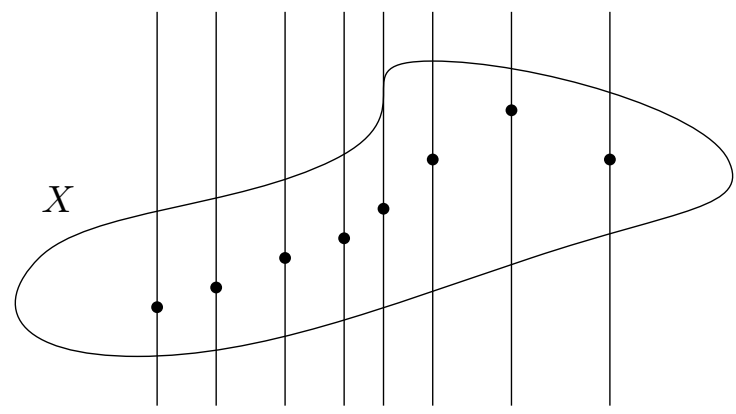


$X$ é o espaço topológico, os pontos representam os germes em $x \in X$ de onde "germinam" os stalks (caules/hastes) representados pelas linhas. O termo "étale" vem do francês e pode ser traduzido como estender ao longo de uma superfície, é como se estivessemos espalhando os stalks $\mathscr{F}_{x}$ ao longo de $X$ de forma a obter o espaço étale $\Lambda_{\mathscr{F}}$. A topologia se encarrega de "amarrar" os stalks formando assim o feixe, que são espigas atadas por uma corda (a topologia).

O teorema a seguir nos diz que todo feixe (no sentido da Definição 2.1.2) é feixe de seção de um fibrado (Definição 2.1.8).

Teorema 2.1.15 Seja $\eta: \mathscr{F} \rightarrow \Gamma\left(\Lambda_{\mathscr{F}}\right)$ a transformação natural tal que para cada aberto $U \in$ obtemos $\eta_{U}: \mathscr{F}(U) \rightarrow \Gamma\left(\Lambda_{\mathscr{F}}\right)(U), \eta_{U}(s)=\dot{s}$. Se $\mathscr{F}$ for um feixe, então $\eta$ é um isomorfismo.

\section{Demonstração:}

Como estamos em Sets, basta ver que é $\eta_{U}$ bijetora. Começamos mostrando que $\eta_{U}$ é injetora. Sejam $s, t \in \mathscr{F}(U)$ e suponha $\dot{s}=\dot{t}$, então germe $x=$ germe $_{x} t, \forall x \in U$. Logo, existem vizinhanças abertas $V_{x}$ de $x$ tais que $V_{x} \subseteq U$ e $s_{\left.\right|_{V_{x}}}=t_{\left.\right|_{V_{x}}}$, e segue que $s$ e $t$ coincidem em cada aberto $V_{x}$ onde $U=\bigcup_{x \in U} V_{x}$ é uma cobertura de $U$. Como $\mathscr{F}$ é feixe, $s=t$. Desta forma, $\eta$ é injetora.

Agora veremos que é sobrejetora. Seja $r \in \Gamma\left(\Lambda_{\mathscr{F}}\right)$, isto é, $r: U \rightarrow \Lambda_{\mathscr{F}}$ uma seção. Para cada $x \in U$ temos $r(x)=$ germe $_{x}\left(s_{x}\right)$, onde $s_{x} \in \mathscr{F}\left(U_{x}\right)$ com $U_{x}$ vizinhança aberta de $x$ e podemos supor $U_{x} \subseteq U$.

Pela definição da topologia, $\dot{s}_{x}\left(U_{x}\right)$ é aberto em $\Lambda_{\mathscr{F}}$. Como $r$ é uma aplicação contínua, o conjunto $r^{-1}\left(\dot{s}_{x}\left(U_{x}\right)\right)=\left\{z \in U \mid r(z)=\operatorname{germe}_{z}\left(s_{x}\right)\right\}$ é uma vizinhança aberta de $x$. Chame $W_{x}=r^{-1}\left(\dot{s}_{x}\left(U_{x}\right)\right)$. Então, para todo $z \in W_{x} \cap W_{y}$, temos germe $e_{z} s_{x}=r(z)=$ germe $_{z} s_{y}$. Como $\mathscr{F}$ é feixe, obtemos $s_{x}=s_{y}$ em $W_{x} \cap W_{y}$.

Considere morfismos $p$ e $q, \quad \prod_{x \in U} \Gamma\left(\Lambda_{\mathscr{F}}\left(\left(W_{x}\right)\right)\right) \stackrel{p}{\longrightarrow} \prod_{x, y \in U}\left(W_{x} \cap W_{y}\right)$.

Temos uma família de elementos $s_{x} \in \mathscr{F}\left(W_{x}\right)$ que possuem mesma imagem quando aplicados em $p$ e $q$. Novamente por propriedade de feixe, existe $s \in \mathscr{F}((U))$ tal que $s_{\left.\right|_{W_{x}}}=$ $s_{x_{\left.\right|_{W}}}$. Então, para cada $x \in U$ vale $r(x)=$ germe $_{x} s_{x}=$ germe $_{x} s=\dot{s}_{x}$, ou seja, $r=\dot{s}$.

Então, dado qualquer $r \in \Gamma\left(\Lambda_{\mathscr{F}}\right)$, escrevemos $r=\eta_{U}(s)$ para algum $s \in \mathscr{F}(U)$. Em outras palavras, $\eta$ é sobrejetora.

Uma pergunta que pode ficar é porque adotar uma noção ou outra de feixe. Pelo que desenvolvemos parece claro que os fibrados fornecem informação mais direta sobre a estrutura topológica/geométrica do objeto com o qual estamos trabalhando, já a definição funtorial nos dá acesso mais direto sobre possíveis estruturas algébricas envolvidas, por exemplo, a cohomologia pode ser vista como um funtor exato à direita, como veremos ao falarmos da cohomologia de feixes e de topos.

A relação entre os fibrados e os feixes é tão íntima que existe uma equivalência entre suas categorias. Partimos de um pré-feixe, construímos um fibrado e encontramos um feixe, se generalizarmos essa ideia podemos definir funtores adjuntos $\Lambda: \operatorname{Sets}^{(X)^{o p}} \rightarrow \operatorname{Et}(X) \mathrm{e}$ $\Gamma: \operatorname{Bund}(X) \rightarrow S h(X)$, onde $\operatorname{Bund}(X)$ é a categoria dos fibrados onde os objetos são funções contínuas com codomínio $X$ e dados dois objetos $p: E \rightarrow X$ e $p^{\prime}: E^{\prime} \rightarrow X$ os morfismos são tais que $\searrow_{x} \swarrow_{p^{\prime}} E^{\prime}$ comuta e $E t(X)$ é categoria dos fibrados étale vista como uma subcategoria plena de $\operatorname{Bund}(X)$. Para detalhes ver o Teorema 2 na Seção 6 do Capítulo II de [MLM94].

A composição $\Gamma \circ \Lambda: \operatorname{Sets}^{(X)^{o p}} \rightarrow S h(X)$ é o que se conhece por funtor de feixificação. Como consequência de $E t(X)$ ser uma subcategoria plena de $\operatorname{Bund}(X)$, passamos a 
ter uma equivalência do tipo

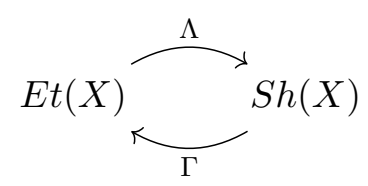

Na próxima seção o funtor de feixificação tornará a aparecer mas em um contexto mais geral, lá apresentaremos uma construção cuidadosa desse funtor que nos dará o processo de feixifação, mas que chamaremos de funtor feixe associado.

Encerramos essa seção com um lema técnico e útil sobre stalks:

Lema 2.1.16 $\quad$ (i) Sejam $\mathscr{F}, \mathscr{G}$ feixes de grupos e $f: \mathscr{F} \rightarrow \mathscr{G}$. Então $f_{x}: \mathscr{F}_{x} \rightarrow \mathscr{G}_{x}$ é injetora para todo $x \in X$ e, e somente se, $f(U): \mathscr{F}(U) \rightarrow \mathscr{G}(U)$ é injetora para todo aberto $U$ de $X$.

(ii) Sejam $\mathscr{F}$ e $\mathscr{G}$ feixes de grupos, se $f_{x}: \mathscr{F}_{x} \rightarrow \mathscr{G}_{x}$ é isomorfismo para todo $x \in X$, então $f: \mathscr{F} \rightarrow \mathscr{G}$ é isomorfismo.

(iii) Seja $\mathscr{F}$ pré-feixe, então $i_{\mathscr{F}}$ induz isomorfismos nos stalks, isto é, $\left(i_{\mathscr{F}}\right)_{x}: \mathscr{F}_{x} \rightarrow \widetilde{\mathscr{F}}_{x}$ é iso.

A demonstração desses resultados pode ser encontrada em [Ive84], no Lema 2.2 e na Proposição 2.4 .

\subsection{Topologias e Topos de Grothendieck e Feixes}

$\mathrm{Na}$ última seção vimos que as ideias de feixes dependem muito da noção de cobertura aberta em um espaço topológico, agora veremos que é possível generalizar esse ideia com algo que imita o papel dos abertos e define uma noção alternativa de cobertura usando nada além de morfismos que seguem certos axiomas, essa será a Topologia de Grothendieck - é ela quem possibilitou definir a Cohomologia Étale de um esquema. Novamente, damos continuidade ao trabalho anterior nos guiando pela mesma referência [MLM94].

Sendo mais específico, antes estávamos definindo feixe em abertos de um certo espaço topológico. Agora poderemos definir feixe numa categoria (pequena) qualquer desde que esteja munida com uma Topologia de Grothendieck.

Estamos em um bom momento para definir o mergulho de Yoneda e enunciar o Lema de Yoneda, mas sem demonstrá-lo. É um resultado famoso utilizado amplamente em teoria de categorias. Uma referência com uma indicação da demonstração é [ML98].

Vamos construir esse mergulho. Seja $\mathcal{C}$ uma categoria pequena e $C$ objeto de $\mathcal{C}$ fixado. Para cada $D$ objeto de $\mathcal{C}$ fazemos $y(C)(D)=\operatorname{Hom}_{\mathcal{C}}(C, D)$ e fixado $f: D^{\prime} \rightarrow D$ morfismo em $\mathcal{C}$, para cada $g: C \rightarrow D^{\prime}$ fazemos $y(C)(f)(g)=f \circ g$. Em outras palavras, $y(C)=$ $\operatorname{Hom}_{\mathcal{C}}(-, C)$.

Definição 2.2.1 Um pré-feixe é dito representável se é isomorfo a $y(C)=H_{o} m_{\mathcal{C}}(-, C)$.

Muitas vezes usa-se $h_{C}$ para denotar o funtor representável.

Agora observe que se considerarmos um morfismo $h: A \rightarrow B$ em $\mathcal{C}$ obtemos uma transformação natural $y(h)=\operatorname{Hom}_{\mathcal{C}}(-, A) \rightarrow \operatorname{Hom}_{\mathcal{C}}(-, B)$, ou seja, $y$ é um funtor.

Definição 2.2.2 O mergulho de Yoneda é o funtor $y: \mathcal{C} \rightarrow \operatorname{Sets}^{{ }^{\circ o p}}$, tal que $y(C)=$ $\operatorname{Hom}_{\mathcal{C}}(-, C)$

Lema 2.2.3 (Lema de Yoneda) Seja $\mathcal{C}$ categoria pequena e $P \in S e t s^{\mathcal{C}^{o p}}$ um pré-feixe. Então existe um isomorfismo canônico $\operatorname{Hom}_{\text {Sets }^{\mathcal{c}}{ }^{o p}}(y(C), P) \cong P(C)$.

O conceito a seguir é um subfuntor do mergulho de Yoneda aplicado em um objeto $C$ de $\mathcal{C}$ 
Definição 2.2.4 Seja $C$ objeto de $\mathcal{C}$. Um crivo em um objeto $C \in \mathcal{C}$ é uma coleção $S$ de morfismos em $\mathcal{C}$ com codomínio $C$ tal que $f \in S$ implica que $f \circ g \in S$, para qualquer morfismo $g$ que tenha como codomínio o domínio de $f$.

De certa forma, $S$ se comporta como um ideal à direita.

Se $S$ é um crivo em $C$ e $h: D \rightarrow C$ é um morfismo qualquer para $C$, então o pullback de $S$ ao longo de $h$ é dado por $h^{*}(S)=\{g \mid \operatorname{cod}(g)=D, h g \in S\}$ é um crivo em $D$. (Usamos "cod" para denotar codomínio e usaremos "dom" para denotar domínio). Também dizemos que $S$ é um crivo gerado por $\left\{f_{i}: C_{i} \rightarrow C\right\}_{i \in I}$ se $S$ é a família de todos os morfismos com codomínio $C$ que se fatora por algum $f_{i}: C_{i} \rightarrow C$.

Veja que, de fato, crivos são subfuntores de $y(C)$. A partir de um crivo construímos um subfuntor e vice-versa: Dado um crivo $S$ em $C$, para cada $D$ defina $P(D)=S \cap$ $H_{\mathcal{C}}(D, C)$. Isso é um subfuntor de $y(C)$. Reciprocamente, tome $P$ subfuntor de $y(C)$ e defina $S=\{f \mid \exists D$ com $f \in P(D)\}$. Resta verificar que $S$ é crivo.

Tome $f: C^{\prime} \rightarrow C$ em $S$ e $g: D \rightarrow C^{\prime}$, dessa forma podemos fazer a composição $f \circ g$ e ela pertence a $P(D)$, logo, $S$ é crivo.

Antes de definirmos uma noção de cobertura para morfismo, devemos exemplificar como os crivos fazem o papel dos abertos de um espaço topológico

Exemplo 2.2.5 Se $\mathcal{C}=\mathcal{O}(X)$, onde $X$ é espaço topológico, o crivo canônico associado ao aberto $U$ de $X$ é dado por $S_{U}=\{V \mid V \subseteq U\}$. Veja que se $V \in S$ e $W$ é um aberto de $X$ contido em $V$, então $W$ está contido em $U$ e, portanto $W \in S_{U}$.

Definição 2.2.6 Uma topologia de Grothendieck em $\mathcal{C}$ é uma função $J$ que leva cada objeto $C \in \mathcal{C}$ para uma coleção $J(C)$ de crivos em $C$ de forma que os seguintes axiomas são satisfeitos:

1. O crivo maximal $\{f \mid \operatorname{cod}(f)=C\}$ está em $J(C)$;

2. (Estabilidade) Se $S \in J(C)$, então $h^{*}(S) \in J(D)$ para todo $h: D \rightarrow C$;

3. (Caráter Local) Se $S \in J(C)$ e $R$ é um crivo em $C$ tal que $h^{*}(R)$ está em $J(D)$ para todo $h: D \rightarrow C$ em $S$, então $R \in J(C)$.

Os elementos de $J(C)$ são chamados crivos de cobertura.

Dando continuidade ao Exemplo 2.2.5, se para cada aberto $U$ de $X$ definirmos $J(U)=$ $\left\{S \mid S\right.$ é crivo em $U$ e $\left.\bigcup_{f \in S} \operatorname{dom} f=U\right\}$, teremos que uma topologia $X$ induz uma topologia de Grothendieck $J$ em $\mathcal{C}=\mathcal{O}(X)$.

Vamos enunciar três execícios breves e resolver um deles para entender como trabalhar com a definição de crivo. Os outros serão uteis em algumas demonstrações.

Exercício 2.2.7 $\quad$ a) Seja $S \in J(C)$, então $R \in J(C)$ para qualquer crivo em $C$ maior que $S$, isto é, $S \subseteq R$.

b) $(J(C), \subseteq)$ é um filtro no conjunto dos crivos em $\mathcal{C}$ (isto é, o crivo maximal está em $J(C)$; se $S \in J(C)$ e $S \subseteq R$, então $R \in J(C)$; se $R_{1}, R_{2} \in J(C)$, então $R_{1} \cap R_{2} \in$ $J(C))$.

c) Seja $S \in J(C)$ e para cada $f \in S$ considere $R_{f} \in J(D)$, então $T=\{f \circ g \mid f \in$ $\left.S, g \in R_{f}\right\} \in J(C)$.

Para a) Basta tomar $h: D \rightarrow C, h \in S$. Assim $1_{D} \in h^{*}(S)$ é um crivo maximal em $D$. Como $h^{*}(S) \subseteq h^{*}(R)$, vale que $h^{*}(R)$ também é crivo maximal em $D$. Logo, $h^{*}(R) \in J(D)$. Temos isso para todo $h \in S$, usando caráter local enunciado acima, $R \in J(C)$.

Note que em b) resta provar que $R_{1} \cap R_{2} \in J(C)$ ) e o item c) é consequência do a). 
Definição 2.2.8 $\mathrm{Um}$ casal $^{1}$ é um par $(\mathcal{C}, J)$, onde $\mathcal{C}$ é uma categoria pequena e $J$ é uma Topologia de Grothendieck.

Definição 2.2.9 Seja $\mathcal{C}$ uma categoria pequena com pullbacks. Uma base para uma topologia de Grothendieck é uma função $K$ que associa cada objeto $C$ de $\mathcal{C}$ uma coleção $K(C)$ de famílias de morfismos $\left\{C_{i} \rightarrow C \mid i \in I\right\}$ tal que:

1. A identidade $i d_{C}: C \rightarrow C$ está em $K(C)$

2. (Estabilidade) Se $\left\{f_{i}: C_{i} \rightarrow C \mid i \in I\right\} \in K(C)$ e $g: D \rightarrow C$ é um morfismo qualquer, então a família dos pullbacks $\left\{g_{i}: C_{i} \times_{C} D \rightarrow D \mid i \in I\right\} \in K(D)$;

3. (Transitividade) Se $\left\{f_{i}: C_{i} \rightarrow C \mid i \in I\right\} \in K(C)$ e para cada $i \in I$ temos uma família $\left\{g_{i j}: D_{i j} \rightarrow C_{i} \mid j \in I_{i}\right\} \in K\left(C_{i}\right)$, então a família das composições $\left\{f_{i} \circ g_{i j}: D_{i j} \rightarrow C \mid i \in I, j \in I_{i}\right\} \in K(C)$.

Os elementos de $K(C)$ são chamados K-coberturas.

Os pares do tipo $(\mathcal{C}, K)$ também são ditos casais.

Note que uma $K$-cobertura falha na propriedade 1 de $J$-cobertura, desta forma, $J$-coberturas e $K$-coberturas não coincidem, porém, dizemos que a base $K$ gera a topologia $J$ através da seguinte equivalência:

$$
S \in J(C) \stackrel{(\star)}{\Longleftrightarrow} \exists R \subseteq S \quad R \in K(C)
$$

Em outras palavras, um crivo $S \in C$ é $J$-cobertura se, e somente se, $S$ contem uma $K$-cobertura. Vamos mostrar que de fato, com essa equivalência, definimos uma topologia de Grothendieck $J$ a partir da base $K$.

1. Seja $R \in K(C)$. O crivo com todos os morfismos com codomínio $C$ é o que chamamos de crivo maximal em $C$ e é claro que ele contem $R$. Por $(\star)$, temos que o crivo maximal está em $J(C)$.

2. Dados um crivo $S \in J(C)$ e um morfismo $g: D \rightarrow C$ escolha $R \subseteq S$ tal que $R \in K(C)$.

Para cada $f_{i}: C_{i} \rightarrow C$ em $R$ tomamos o pullback de $f_{i}$ com $g$ e temos uma família $T=\left\{g_{i}: C_{i} \times_{C} D \rightarrow D\right\} \in K(D)$. Veja ainda que $\operatorname{cod}\left(g_{i}\right)=D$ e $g \circ g_{i} \in S$, logo, $T \subseteq g^{*}(S)$. Por $(\star)$, concluímos que $g^{*}(S) \in J(C)$.

3. Seja $S \in J(C)$ e $h: D \rightarrow C$ um morfismo qualquer. Usando a equivalência ( $\star$ ) temos que existem $R_{S} \in K(C)$ com $R_{S} \subseteq S$. Veja que $h^{*}\left(R_{S}\right) \in J(D)$. Com efeito, se considerarmos $T=\left\{g: D \times_{C} C_{i} \rightarrow D\right\} \in K(D)$, obtido pelo pullback de $R_{S}$ ao longo de $h$ temos, para algum $f_{i} \in R_{S}$ :

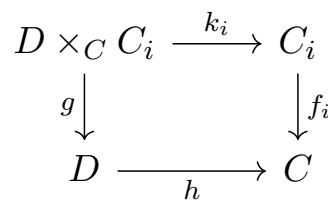

Assim, $h \circ g=f_{i} \circ k_{i}$. Como $f_{i} \in R_{S}$ e $R_{S}$ é crivo, $h \circ g \in R_{S}, \log$ o, $g \in h^{*}\left(R_{S}\right)$. Então $T \subseteq h^{*}\left(R_{S}\right)$. Por $(\star), h^{*}\left(R_{S}\right) \in J(D)$.

Agora faça $T=\left\{g: C_{i} \times_{C} C_{i} \rightarrow C_{i}\right\} \in K\left(C_{i}\right)$. Pela transitividade, a família das composições $Q=\left\{f_{i} \circ g \mid f_{i} \in R_{S}, g \in T\right\} \in K(C)$. Como $R_{S}$ é crivo, $Q \subseteq R_{S}$. Portanto, por $(\star), R_{S} \in J(C)$. Assim provamos o caráter local.

\footnotetext{
${ }^{1}$ Do inglês "site", em espanhol é comum usarem "sítio" como tradução e poderíamos repetí-la em português, contudo, nenhum dos casos nos pareceu adequado. Optamos por "casal"pela ambiguidade apropriada - como o que é formado por um par ou no sentido de propriedade rural, mantendo assim a nomenclatura pastoril bastante presente na teoria de feixes.
} 
Agora vamos nos preparar para definir feixes de um casal.

Recapitulando, dada uma categoria pequena $\mathcal{C}$, um pré-feixe $P$ é um funtor $P: \mathcal{C}^{o p} \rightarrow$ Sets.

Vamos utilizar uma noteção em que o ponto ". " indica a restrição de um elemento $y \in P(D)$ ao longo de um morfismo $g \in \mathcal{C}$ com codomínio $D$. Escrevemos $y \cdot g=P(g)(y)$. Note que estão satisfeitas as condições:

1. $y \cdot i d=y$

2. Se $H \stackrel{h}{\rightarrow} E \stackrel{g}{\rightarrow} D$, enão $y \cdot(g \circ f)=(y \cdot g) \cdot h$.

Sejam $f: D \rightarrow C$ e $g: E \rightarrow D$ morfismos em $\mathcal{C}$. Temos $x_{f} \in P(D)$ e fazemos:

$$
\begin{gathered}
P(D) \stackrel{P(g)}{\longmapsto} P(E) \\
x_{f} \longmapsto x_{f g}
\end{gathered}
$$

Observe que, em geral, se $y \in P(D)$, o domínio dos morfismos $g$ também pode variar, apenas o contradomínio deve ser igual ao domínio da $f$ para que a composição faça sentido.

Com isso bem entendido apresentamos as definições de família compatível, amalgamação e feixe para uma topologia de Grothendieck.

Definição 2.2.10 Seja $P$ um pré-feixe e $S$ um crivo de um objeto $C$ de $\mathcal{C}$. Uma família compatível para $S$ de elementos de $P$ é uma função que leva cada elemento $f: D \rightarrow C$ de $S$ em um elemento $x_{f}$ de $P(D)$ de forma que:

$$
x_{f} \cdot g=x_{f g} \text {, para todo morfismo } g: E \rightarrow D \text { em } \mathcal{C} .
$$

Como $S$ é crivo, a composição $f g$ está em $S$ também.

Definição 2.2.11 Uma amalgamação de uma família compatível é um elemento $x$ de $P(C)$ com $x \cdot f=x_{f}$ para todo morfismo $f$ em $S$.

Definição 2.2.12 Seja $J$ uma topologia de Grothendieck em $\mathcal{C}$. O pré-feixe $P$ é um feixe para $J$ (ou $J$-feixe) se, para qualquer cobertura de qualquer objeto de $\mathcal{C}$, toda família compatível possui uma única amalgamação.

Notação: Usamos $S h(\mathcal{C}, J)$ para designar a categoria dos feixes sobre $(\mathcal{C}, J)$. Os objetos são $J$-feixes e os morfismos são transformações naturais.

A definição acima é análoga à noção de feixe apresentada na seção anterior. A amalgamação faz o papel da propriedade de colagem e, quando ela é única, obtemos o feixe, da mesma forma como temos a unicidade da colagem para feixes de conjuntos.

Podemos também expressar a definição através de um diagrama de equalizador. Seja $C$ objeto de $\mathcal{C}$ e $S$ um crivo em $J(C)$.

$$
P(C) \stackrel{e}{\longrightarrow} \prod_{f} P(\operatorname{dom} f) \stackrel{p}{\underset{a}{\longrightarrow}} \prod_{(f, g)} P(\operatorname{domg})
$$

onde $f \in S$, dom $f=\operatorname{codg}, e(x)=(x \cdot f)_{f \in S}=(P(f)(x))_{f \in S}$. O morfismo $p$ atua nas composições fazendo $p(\bar{x})_{f, g}=x_{f g}$ e $a$ se comporta como uma ação de $\mathcal{C}$ em $P a(\bar{x})_{f, g}=$ $x_{f} \cdot g=P(g)\left(x_{f}\right), \operatorname{com} \bar{x}=\left(x_{f}\right)_{f} \in \prod_{f \in S} P(\operatorname{dom} f)$

Definição 2.2.13 Se o diagrama acima é um equalizador dizemos que o pré-feixe $P$ satisfaz a condição de feixe com respeito a cobertura $S$. 
Se tivermos $K$ uma base para $\mathcal{C}$ que gera uma topologia de Grothendieck $J$, podemos também descrever os feixes para $J$ pelos termos da sua base $K$. Seja $R \in K(C), C$ objeto de $\mathcal{C}$, isto é, R é uma K-cobertura e como tal é da forma $R=\left\{f_{i}: C_{i} \rightarrow C \mid i \in I\right\}$.

Uma família de elementos $\left\{x_{i} \in P\left(C_{i}\right) \mid i \in I\right\}$, é compatível a $R$ se, e somente se, $x_{i} \cdot \pi_{i j}^{1}=x_{j} \cdot \pi_{i j}^{2}, \forall i, j \in I$, onde $\pi_{i j}^{1}$ e $\pi_{i j}^{2}$ são as projeções do pullback

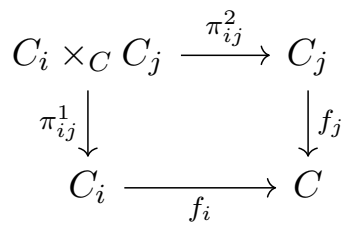

A amalgamação para $\left\{x_{i}\right\}_{i \in I}$ é um $x \in P(C)$ tal que $x \cdot f_{i}=x_{i}, \forall i \in I$.

Proposição 2.2.14 Seja $P$ um pré-feixe em $\mathcal{C}$. Então $P$ é um feixe para $J$ se, e somente se, para qualquer cobertura $\left\{f_{i}: C_{i} \rightarrow C \mid i \in I\right\}$ na base $K$, toda família compativel $\left\{x_{i}\right\}_{i \in I}$ possui uma única amalgamação.

\section{Demonstração:}

( $\Longrightarrow$ ) Suponha $P$ um feixe para $J$. Seja $R=\left\{f_{i}: C_{i} \rightarrow C \mid i\right\} \in I$ uma $K$-cobertura. Seja $\left\{x_{i}\right\}_{i \in I}$ a família compatível dessa cobertura.

Considere o crivo $S=\left\{g: D \rightarrow C \mid g=D \stackrel{h_{i}}{\longrightarrow} C_{i} \stackrel{f_{i}}{\longrightarrow} C\right.$ para algum $i$ e $\left.h_{i}\right\}$ gerado por $R$. Defina uma família para $S,\left\{y_{g}\right\}_{g \in S}$, onde $y_{g}=x_{i} \cdot h_{i}$ com $h$ tal que $g=f_{i} \circ h_{i}$ para algum $i \in I$.

Note que $f_{i} \circ h_{i}=g=f_{j} \circ h_{j}$, então $g$ não depende da escolha nem de $i$ nem de $h_{i}$.

Pela propriedade universal do diagrama de pullback abaixo, existe um mapa $l$ onde $\pi_{i j}^{1} \circ l=h_{i}$ e $\pi_{i j}^{2} \circ l=h_{j}$.

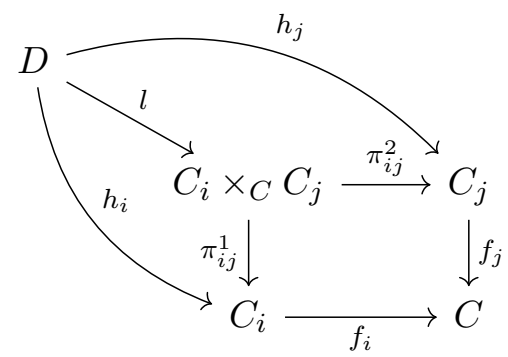

$$
\begin{array}{rlrl}
x_{i} \cdot h_{i} & =\left(x_{i} \cdot \pi_{i j}^{1}\right) \cdot l & & \text { Pois } \pi_{i j}^{1} \circ l=h_{i} \\
& =\left(x_{j} \cdot \pi_{i j}^{2}\right) \cdot l & & \text { Pois }\left\{x_{i}\right\} \text { é família compatível de R, } \\
& =x_{j} \cdot k & & \text { isto é, o diagrama comuta } \\
& & \text { Pois } \pi_{i j}^{2} \circ l=h_{j}
\end{array}
$$

Como $P$ é feixe em $J$, existe um único $y \in P(C)$ tal que $y \cdot g=y_{g}, \forall g \in S$.

Em particular, uma vez que $f_{i} \in S$, temos $y \cdot f_{i}=y_{f_{i}}=x_{i}$ (pois $f_{i} \in R$ e $x_{i}$, $f_{i}=f_{i} \circ i d_{C_{i}}$ e $\left.x_{i} \cdot i d_{C_{i}}=x_{i}\right)$.

Sendo assim, $y$ é uma amalgamação para $\left\{x_{i}\right\}_{i \in I}$. Falta ver que é única.

Suponha que exista uma outra amalgamação $y^{\prime}$ para $\left\{x_{i}\right\}_{i \in I}$. Temos então $y^{\prime} \cdot f_{i}=x_{i}$. Logo, para todo $g \in S$, vamos chamar $g=f_{i} \circ h_{i}$, fazemos

$$
y^{\prime} \cdot g=y^{\prime} \cdot f_{i} \cdot h_{i}=x_{i} \cdot h_{i}=y_{g} \text {, por definição de } y_{g} .
$$

Assim temos que $y^{\prime}$ é uma amalgamação para $S$, mas em $S$ é única pois $P$ é feixe em $J$. 
$(\Longleftarrow)$ Seja $S \in J(C)$ uma cobertura de $C$ tal que existe $R \in K(C)$ uma $K$-cobertura de $C$ contida em $S$, isto é, $R \subseteq S$.

Seja $\left\{y_{g} \mid g \in S\right\}$ uma família compatível para $S$. Como $R \subseteq S$, se tomarmos uma família $\left\{y_{f} \mid f \in R\right\}$, teremos que ela é compatível para $R$.

De fato, temos $R=\left\{f_{i}: C_{i} \rightarrow C \mid i \in I\right\}$ e o diagrama de pullback dado anteriormente. Chame $\delta=f_{i} \circ \pi_{i j}^{1}=f_{j} \circ \pi_{i j}^{2}$. Como $f_{i}$ e $f_{j}$ pertencem a $S$, temos $\delta \in S$. Com $\left\{y_{g} \mid g \in S\right\}$ é compatível para $S$, temos

$$
y_{\delta}=y_{f_{i} \circ \pi_{i j}^{1}}=y_{f_{i}} \cdot \pi_{i j}^{1} \text { e } y_{\delta}=y_{f_{j} \circ \pi_{i j}^{2}}=y_{f_{j}} \cdot \pi_{i j}^{2}
$$

Então $\left\{y_{f} \mid f \in R\right\}$ é compatível para $R$.

Por hipótese, a amalgamação é única, então existe um único $y \in P(C)$ tal que $y \cdot f=$ $y_{f}, \forall f \in R$.

Resta mostrar que $y \cdot g=y_{g}, \forall g \in S$.

Seja $g \in S$, para cada $f \in R$ temos o pullback $\begin{gathered}D \times_{C} C^{\prime} \stackrel{\pi_{f, g}^{2}}{\longrightarrow} C^{\prime} \\ \quad \pi_{f, g}^{1} \downarrow \\ D \longrightarrow\end{gathered}$ estabilidade na base da topologia de Grothendieck temos que $R^{\prime}=\left\{\pi_{f, g}^{1} \mid f \in R\right\} \in K(D)$.

Além disso, para todo $f$ em $R$,

$$
\begin{aligned}
(y \cdot g) \cdot \pi_{f, g}^{1} & =(y \cdot f) \cdot \pi_{f, g}^{2} & & \text { Pois o diagrama comuta } \\
& =y_{f} \cdot \pi_{f, g}^{2} & & \text { Pois } f \in R \\
& =y_{f \circ \pi_{f, g}^{2}} & & \text { Pois }\left\{y_{g} \mid g \in R\right\} \text { é compatível } \\
& =y_{g \circ \pi_{f, g}^{1}} & & \text { Pois o diagrama comuta } \\
& =y_{g} \cdot \pi_{f, g}^{1} & &
\end{aligned}
$$

Considere $f^{\prime}: C^{\prime \prime} \rightarrow C$ em $R$, se tomarmos o pullback de $\pi_{f, g}^{1}$ ao longo de $\pi_{f^{\prime}, g}^{1}$ e repetirmos as contas acima, obteremos $y \cdot g \cdot \pi_{f, g}^{1}$ compatível com $y \cdot g \cdot \pi_{f^{\prime}, g}^{1}$, pois grudar diagramas de pullback dá outro diagrama de pullback (Lema do Pullback).

Como $(y \cdot g) \cdot \pi_{f, g}^{1}=y_{g} \cdot \pi_{f, g}^{1}$, temos que $\left\{y \cdot g \cdot \pi_{f, g}^{1} \mid f \in R\right\}$ é compatível de $R^{\prime}$ e possui uma única alamagação.

Mas note que nesse processo encontramos duas almagamações para $\left\{y \cdot g \cdot \pi_{f, g}^{1} \mid f \in\right.$ $R\}$. São elas $y_{g}$ e $y \cdot g$, pois $y \cdot g \cdot \pi_{f, g}^{1}=y_{g} \cdot \pi_{f, g}^{1}$ e, claro, $y \cdot g \cdot \pi_{f, g}^{1}=(y \cdot g) \cdot \pi_{f, g}^{1}$. Como a amalgamação é única, concluímos $y_{g}=y \cdot g$

Trabalhamos bastante com feixes de uma topologia de Grothendieck, agora é um bom momento para definirmos um Topos de Grothendieck.

Definição 2.2.15 Um topos de Grothendieck é uma categoria que seja equivalente à categoria $S h(\mathcal{C}, J)$ para algum casal $(\mathcal{C}, J)$.

Dependendo do caso, há formas naturais de descrever o casal $(\mathcal{C}, J)$ de um topos de Grothendieck, mas nem sempre isso é uma tarefa fácil. Por exemplo, em [MLM94] uma seção inteira (9 - Capítulo III) é dedicada para exibir o casal da categoria dos $G$-sets contínuos, onde $G$ é um grupo topolológico. Contudo, existe outra forma de identificar um topos de Grothendieck - através do conhecido Teorema de Giraud. Vamos falar sobre ele mais tarde.

Agora vamos mostrar um importante resultado: limites de um diagrama de feixes é feixe. 
Proposição 2.2.16 Seja $(\mathcal{C}, J)$ um casal e $I \rightarrow$ Set $^{\mathcal{C}^{o p}}$ um diagrama de pré-feixe $P_{i}$. Se todo $P_{i}$ é feixe, então $\varliminf_{i} P_{i}$ também é feixe.

\section{Demonstração:}

Seja $I \rightarrow S e t s^{\mathcal{C}^{o p}}$ um diagrama de pré-feixes que leva cada índice $i$ em pré-feixe $P_{i}$, então o limite inverso em ets $^{\mathcal{C}^{o p}}$ é calculado ponto por $\left(\varliminf_{C} P_{i}\right)(C)=\varliminf_{\mathcal{C}}\left(P_{i}(C)\right)$, sendo que do lado direito estamos usando o limite de conjuntos e $C \in \mathcal{C}$.

Faça $P=\lim _{\longleftarrow} P_{i}$ o limites em $\operatorname{Sets}^{\mathcal{C}^{o p}}$, assim $P(C)=\left(\varliminf_{\longleftarrow} P_{i}\right)(C)$ iremos mostrar que $P$ é feixe.

Dada uma cobertura $S$ de $C$, como cada $P_{i}$ é feixe, temos que o seguinte diagrama é um equalizador $P_{i}(C) \longrightarrow \prod_{f \in S} P_{i}(\operatorname{dom} f) \Longrightarrow \prod_{f, g \in S} P_{i}(\operatorname{domg}) \quad \forall i \in I$.

Como limites comutam entre si, se tormarmos o limite inverso do diagrama de equalizador, obtemos que $P(C) \longrightarrow \prod_{f \in S} P(\operatorname{dom} f) \longrightarrow \prod_{f, g \in S} P(d o m g)$ é um equalizador.

Portando, $P$ é feixe.

Os morfismo da categoria $S h(\mathcal{C}, J)$ são transformações naturais entre pré-feixes, de forma que $S h(\mathcal{C}, J)$ é uma subcategoria plena de $S e t s^{\mathcal{C}^{o p}}$, então temos um funtor inclusão $i: \operatorname{Sh}(\mathcal{C}, J) \rightarrow \operatorname{Sets}^{\mathcal{C}^{o p}}$.

Vamos construir um funtor $a: \operatorname{Sets}^{\mathcal{C}^{o p}} \rightarrow S h(\mathcal{C}, J)$, que é adjunto à esquerda desse funtor inclusão.

Vemos que através do funtor $a$, dado um pré-feixe este consegue nos fornecer um feixe. Esse processo é conhecido como feixificação e muitas vezes $a$ é chamado de funtor feixe associado. Sim, mencionamos algo similar quando vimos feixes de seção de um fibrado.

A construção será feita em 4 etapas. Primeiro vamos definir um novo funtor, o pré-feixe $P^{+}$. Depois veremos que $P^{+}$é um pré-feixe separável (a ideia de separável aqui é de um pré-feixe que é quase um feixe). A terceira etapa consiste em verificar que se $P$ já era separável, então $P^{+}$é feixe. Por fim, definiremos $a(P)=\left(P^{+}\right)^{+}$, desta forma a cumpre com as funções que esperamos.

Dito isso, vamos tornar preciso o que queremos dizer com um pré-feixe que é "quase"um feixe.

Definição 2.2.17 Um pré-feixe $P$ é dito separável se as família compatíveis possuem, no máximo, uma amalgamação.

Ou seja, garantimos a unicidade mas não a existência de almagamações. Iniciaremos agora com a primeira etapa da construção do funtor $a$.

Usaremos $\operatorname{Comp}(R, P)$ para designar o conjunto das famílias compatíveis da cobertura $R$. Para definir $P^{+}$teremos que tomar o colimite, sobre todas as coberturas de $C$, dessas famílias compatíveis. Em outras palavras:

Definição 2.2.18 Seja $P$ um pré-feixe qualquer, definimos $P^{+}=\underset{R \in J(C)}{\lim _{\longrightarrow}} \operatorname{Comp}(R, P)$

Dessa forma $(+)$ pode ser visto como um funtor, chamado de "semi-feixificação". O motivo desse nome ficará claro com os próximos passos.

Essa definição pode parecer artificial, mas ela faz sentido. Temos $(J(C), \subseteq)$ um poset, se $P$ fosse feixe, teríamos que para cada cobertura $R$ de um objeto $C$ em $\mathcal{C}$ uma família compatível $\left\{x_{f}\right\}_{f \in R}$ de elementos de $P$ representam um único elemento de $P(C)$. Mas se considerarmos um refinamento $S$ de $R$, teremos que a subfamília $\left\{x_{f}\right\}_{f \in S}$ representa o mesmo elemento de $P(C)$. Desta forma, é natural pensar em tomar os colimites de todos os crivos de cobertura de $C$, ordenados por inclusão inversa.

Note que um elemento de $P^{+}(C)$ é uma classe de equivalência de famílias compatíveis. Seja $f: D \rightarrow C$ de $R$. Considere $\dot{x}=\left\{x_{f} \mid f \in R\right\}$, onde $x_{f}$ pertence a $P(D)$ e $x_{f} \cdot k=$ 
$x_{f k}$ para todo $k: E \rightarrow D$. Seja $\dot{y}=\left\{y_{g} \mid g \in S\right\}$, temos que $\dot{x}$ e $\dot{y}$ são equivalentes quando existe um refinamento comum $T \subseteq R \cap S, T \in J(C)$ tal que $x_{h}=y_{h}$ para todo $h \in T$.

Recordamos que $h^{*} R=\left\{g \mid \operatorname{cod} g=C^{\prime}, h g \in R\right\}$

Seja $h: C^{\prime} \rightarrow C$ em $\mathcal{C}$, o mapa restrição $P^{+}(C) \rightarrow P^{+}\left(C^{\prime}\right)$ é dado por

$$
\left\{x_{h f^{\prime}} \mid f^{\prime} \in h^{*} R\right\}=P^{+}(h)\left(\left\{x_{f}\right\}_{f \in R}\right)
$$

e está bem definido nas classes de equivalência pois dados y e $T$ como acima, então $h^{*}(T) \subseteq$ $h^{*}(R) \cap h^{*}(S)$ e $x_{h f^{\prime}}=y_{h f^{\prime}}$, para $f^{\prime} \in h^{*}(T)$.

Isso nos diz que $P^{+}$possui estrutura de pré-feixe.

Considere $\phi: P \rightarrow Q$ um mapa entre pré-feixes, temos que $\phi$ induz um mapa $\phi^{+}$: $P^{+} \rightarrow Q^{+}$, onde $Q^{+}=\underset{R \in J(C)}{\lim _{R}} \operatorname{Comp}(R, Q)$, pois $\phi$ induz uma aplicação $\phi$ nas famílias compatíveis $\phi^{\prime}: \operatorname{Comp}(R, P) \rightarrow \operatorname{Comp}(R, Q),\left\{x_{f}\right\}_{f} \mapsto\left\{\phi\left(x_{f}\right)\right\}_{f}$.

Então $P \mapsto P^{+}$é um funtor de $P$, mais do que isso, temos uma transformação natural canônica $\eta: P \rightarrow P^{+}$em que $\eta_{C}(x)=\left\{x \cdot f \mid f \in t_{C}\right\}$ para todo $x \in P(C)$, onde $t_{C}=\{f \mid \operatorname{codf}=C\}$ é o crivo maximal.

Isso é o suficiente para considerarmos encerrada a primeira etapa e darmos início à segunda. Enunciaremos e demonstraremos 3 proposições para verificar que $P^{+}$é separável.

Proposição 2.2.19 Valem as seguintes afirmações:

(i) Um pré-feixe $P$ é separável se, e somente se, $\eta: P \rightarrow P^{+}$é um monomorfismo.

(ii) Um pré-feixe $P$ é feixe se, e somente, $\eta: P \rightarrow P^{+}$é um isomorfismo

\section{Demonstração:}

(i) Sejam $x, y \in P(C)$ e $f: D \rightarrow C$ em $\mathcal{C}$

$(\Longrightarrow)$ Temos que $x \cdot f=y \cdot f$ implica $x=y$, pois $x$ e $y$ são amalgamações de $\left\{x_{f}\right\}_{f}$ e $P^{+}$é separável.

Logo, $\eta_{C}(x)=x \cdot f=y \cdot f=\eta_{C}(y)$ implica $x=y$. Então $\eta$ é monomorfismo.

$(\Longleftarrow)$ Temos que $\eta_{C}(x)=\eta_{C}(y)$ implica $x=y$, mas $\eta_{C}(x)=\{x \cdot f\}_{f}$ e $\eta_{C}(y)=$ $\{y \cdot f\}_{f}$.

Seja $\left\{z_{f}\right\}_{f}$ uma família compatível e suponha que existam duas amalgamações $x$ e $y$ de $\left\{z_{f}\right\}_{f}$ Então $x \cdot f=z_{f}=y \cdot f$, ou seja, a amalgamação, se existir, é única. Portanto, $P^{+}$é pré-feixe separável.

(ii) $(\Longrightarrow)$ Seja $\left\{x_{f}\right\}_{f \in R}$ família compatível para $R$ e $x_{f}=x . f$ para todo $f \in R$.

Se tivermos $y \cdot f=x_{f}$ para todo $f \in R$, com $P$ é feixe, $x=y$ (a amalgamação é única).

Agora note que $R \subseteq R \cap t_{C}$, logo, é um refinamento comum de $R$ e $t_{C}$. Então obtemos $\left\{x_{f}\right\}_{f \in R}=\left\{y_{f}\right\}_{f \in t_{C}}=\eta_{C}(y)$, logo, $\eta_{C}$ é sobrejetora. Como $\eta$ é monomorfismo por (i), concluímos que $\eta$ é isomorfismo.

$(\Longleftarrow)$ Se $\eta$ é isomorfismo, em particular, é mono. Então já temos que $P$ é pré-feixe separável por (i). Resta garantir a existência da amalgamação.

Seja $\left\{x_{f}\right\}_{f \in R}$ uma família compatível para $R$. Como $\eta_{C}$ é sobrejetora, existe $y \in P(C)$ e $S \subseteq R \cap t_{C}=R$ tal que $y \cdot f=x_{f}$ para todo $f \in S$. Assim, $y$ é amalgamação para $\left\{x_{f}\right\}_{f \in S}$ e para $\left\{x_{f}\right\}_{f \in R}$

Concluímos assim que $P$ é feixe. 
Proposição 2.2.20 Se $F$ é um feixe e $P$ é pré-feixe, então qualquer mapa $\phi: P \rightarrow F$ de pré-feixes se fatora unicamente por $\eta, \psi=\widetilde{\phi} \circ \eta$, como representado pelo diagrama

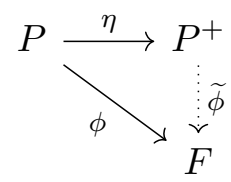

\section{Demonstração:}

Seja $\dot{x}=\left\{x_{f} \mid f \in R\right\}$ elemento de $P^{+}(C), C$ objeto de $\mathcal{C}$ e $R$ cobertura de $C$.

Recorde que $\eta_{C}(x)=\left\{x \cdot f \mid f \in t_{C}\right\}$, assim, dado um morfismo $h: D \rightarrow C$ em $R$, temos $\eta_{D}\left(x_{h}\right)=\left\{x_{h} \cdot k \mid k \in t_{D}\right\}$.

Mas também vale $\left\{x_{h f^{\prime}} \mid f^{\prime} \in h^{*} R\right\}=P^{+}(h)\left(\left\{x_{f}\right\}_{f \in R}\right)$

Observe que $h^{*} R=t_{D}$. Como $t_{D}=\{f \mid \operatorname{codf}=D\}$ e $h^{*} R=\{g \mid \operatorname{codg}=D, h g \in R\}$, é imediato que $h^{*} R \subseteq t_{D}$.

Por outro lado, como $h$ está em $R$, temos $h f \in R$ para qualquer $f$ em $t_{D}$, logo, $f$ também estará em $h^{*} R$. Lembrando que $\dot{x}$ é família compatível obtemos:

$$
\eta_{D}\left(x_{h}\right)=\left\{x_{h k} \mid f \in t_{D}\right\}=\left\{x_{h f^{\prime}} \mid f^{\prime} \in h^{*} R\right\}=\left\{x_{f} \mid f \in R\right\} . h
$$

Queremos definir elemento $y=\widetilde{\phi}(\dot{x})$ de $F(C)$ que satisfaça:

$$
y \cdot h=\widetilde{\phi}(\dot{x}) \cdot h=\widetilde{\phi}\left(\left\{x_{f} \mid f \in R\right\} . h\right)=\widetilde{\phi}\left(\eta_{D}\left(x_{h}\right)\right)=\phi\left(x_{h}\right), \forall h \in R
$$

Fazemos um parênteses aqui caso haja alguma dúvida sobre como passamos $h$ para dentro de $\widetilde{\phi}$. Isso ocorre porque $\widetilde{\phi}(\dot{x})$. $h$ é $F(h)\left(\widetilde{\phi}\left(\dot{x_{f}}\right)\right)$ que por sua vez - pois $\widetilde{\phi}$ deve ser transformação natural entre pré-feixes - é igual a $\widetilde{\phi}\left(\{f\}_{f} \cdot h=\widetilde{\phi}\left(P^{+}(h)\left(\left\{x_{f}\right\}_{f}\right)\right)\right.$

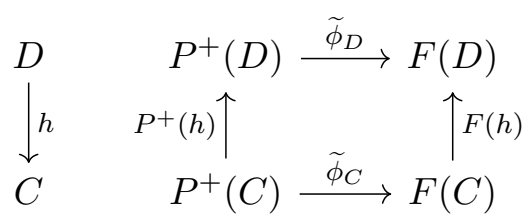

Ou seja, definimos $\widetilde{\phi}_{C}(\dot{x})$ como a única amalgamação de $\left\{\phi\left(x_{h}\right) \mid h \in R\right\}$, onde tal família é compatível de $F$ e define transformação natural.

Dessa forma, para todo $\phi: P \rightarrow F$ encontramos um $\widetilde{\phi}$ tal que $\phi=\widetilde{\phi} \circ \eta$.

Proposição 2.2.21 Seja $P$ pré-feixe, então $P^{+}$é pré-feixe separável.

\section{Demonstração:}

Seja $Q$ uma cobertura de um objeto $C$ de $\mathcal{C}$. Consideremos $z$ e $w$ elementos de $P^{+}(C)$ tais que $z \cdot h=w \cdot h$, para algum $h \in Q$.

Sendo $z$ e $w$ elementos de $P^{+}(C)$, temos que são classes de equivalência de famílias compatíveis da forma $z=\left\{x_{f} \mid f \in R\right\}$ e $w=\left\{y_{g} \mid g \in S\right\}$, com $R$ e $S$ coberturas de $C$.

Agora vamos tomar um refinamento de cobertura que fornece uma família compatível. Seja $h: D \rightarrow C$ em $Q$, a igualdade $z \cdot h=w \cdot h$ nos diz que existe uma cobertura $T_{h}$ de $D$ tal que $T_{h} \subseteq h^{*}(R) \cap h^{*}(S)$ e $z_{h t}=y_{h t}, \forall t \in T_{h}$.

Então, para cada $h \in Q$, onde $Q$ é uma cobertura de $C$, temos umas cobertura $T_{h}$ de $D$ então a família das composições $T=\left\{h t \mid h \in Q, t \in T_{h}\right\}$ é uma cobertura de $C$, pelo Exercício 2.2.7(c), onde $T \subseteq R \cap S$.

Então encontramos um refinamento tal como necessário para definir equivalência entre família compatíveis, logo, $w=z$. Portanto, existirá no máximo uma amalgamação.

Proposição 2.2.22 Seja $P$ pré-feixe separável, então $P^{+}$é um feixe. 


\section{Demonstração:}

Uma vez que $P^{+}$é separável já temos a unicidade, falta verificar a existência da amalgamação para toda família compatível. O que faremos é amalgamar todas as famílias compatíveis de elementos de $P^{+}$.

Seja $R \in J(C)$ e considere a família $\left\{\dot{x}_{f} \mid f \in R\right\}$. Então temos $f: D \rightarrow C$ e $\dot{x}_{f} \in$ $P^{+}(D)$. Vamos trabalhar com as classes de equivalência, isto é, cada $\dot{x}_{f}=\left\{x_{f, g} \mid g \in S_{f}\right\}$ é uma classe de equivalência de famílias compatíveis onde $g: E \rightarrow D, x_{f, g} \in P(E)$ e $S_{f} \in J(D)$.

Como $\left\{\dot{x}_{f} \mid f \in R\right\}$ é compatível, temos que $P^{+}(h)\left(\dot{x}_{f}\right)=\dot{x}_{f h}, \forall h: D^{\prime} \rightarrow D$. Os mapas restrição de $P^{+}(D) \rightarrow P^{+}\left(D^{\prime}\right)$ nos fornecem uma equivalência de famílias

$$
\left\{x_{f h, g} \mid g \in S_{f h}\right\} \sim\left\{x_{f, h g^{\prime}} \mid g^{\prime} \in h^{*}\left(S_{f}\right)\right\}
$$

Isso significa que existe um refinamento $T_{f, h}$ em $J\left(D^{\prime}\right)$ onde $T_{f, h} \subseteq h^{*}\left(S_{f}\right) \cap S_{f h}$ tal que

$$
x_{f, h l}=x_{f h, l} \forall l \in T_{f, h}(\star)
$$

Vamos precisar da igualdade acima mais tarde, por hora, defina $Q=\{f \circ g \mid f \in$ $\left.R, g \in S_{f}\right\}$. Como $R$ e $S_{f}$ são coberturas de $C, Q$ cobre $C$ também (Exercício 2.2.7(c)).

Agora defina $\dot{y}=\left\{y_{f g} \mid f g \in Q\right\} \in P^{+}(C)$ uma família para $Q$ tal que $y_{f g}=x_{f, g}$.

Primeiro devemos mostrar que a definição de $\dot{y}$ não depende da escolha de fatoração de $f g$.

Suponha $f g=f^{\prime} g^{\prime} \operatorname{com} f, f^{\prime} \in R$ e $g \in S_{f}, g^{\prime} \in S_{f^{\prime}}$. Seja $k \in T_{f, g} \cap T_{f^{\prime}, g^{\prime}}$, então:

$$
\begin{aligned}
x_{f, g} \cdot k & =x_{f, g k} & & \text { Pois } \dot{x}_{f} \text { é compatível } \\
& =x_{f g, k} & & \text { Por }(\star) \\
& =x_{f^{\prime} g^{\prime}, k} & & \text { Pois fg }=\mathrm{f}^{\prime} \mathrm{g}^{\prime} \\
& =x_{f^{\prime}, g^{\prime} k} & & \text { Por }(\star) \\
& =x_{f^{\prime}, g^{\prime}} \cdot k & & \text { Como } f^{\prime} \in R, \dot{x}_{f}^{\prime} \text { é compatível }
\end{aligned}
$$

Como $T_{f, g} \cap T_{f^{\prime}, g^{\prime}}$ é uma cobertura de $D^{\prime}$ e $P$ é separável, obtemos $x_{f, g}=x_{f^{\prime}, g^{\prime}}$, logo, $\dot{y}$ está bem definida. Temos ainda que $\dot{y}$ é elemento de $P^{+}(C)$, pois $\left\{\dot{x}_{f} \mid f \in R\right\}$ compatível implica que $\dot{y}$ é compatível.

Resta mostrar que $\dot{y}$ é uma amalgamação de $\left\{\dot{x}_{f} \mid f \in R\right\}$. Queremos verificar que $\dot{y} \cdot f=\left\{y_{f h} \mid h \in h^{*} Q\right\}\left\{\dot{x}_{f}\right\}=\left\{x_{f, g} \mid g \in S_{f}\right\}$ na realidade correspondem ao mesmo elemento de $P^{+}(D)$ para todo morfismo $f: D \rightarrow C$ em $R$.

Por definição de $Q$ rapidamente verificamos que $S_{f}=f^{*}(Q)=\{k \mid \operatorname{codk}=D, f k \in$ $Q\}$. Além disso, por definição de $\dot{y}$, temos $y_{f g}=x_{f, g}, \forall g \in S_{f}, \operatorname{logo}, \dot{y} \cdot f=\left\{y_{f g} \mid g \in\right.$ $\left.S_{f}\right\}=\left\{x_{f, g} \mid g \in S_{f}\right\}=\dot{x}_{f}$.

Então, para cada $f \in R$ construimos $\dot{y} \in P^{+}(C)$ tal que $\dot{y} \cdot f=\dot{x}_{f}$, ou seja, para toda família compatível $\dot{x}_{f}$ encontramos uma amagalmação $\dot{y}$. Vimos na última proposição que $P^{+}$é separável, portanto, essa amalgamação é única. Assim concluímos que $P^{+}$é feixe.

O próximo Teorema cumpre o papel da $4^{\mathrm{a}}$ etapa.

Teorema 2.2.23 O funtor inclusão $i: S h(\mathcal{C}, J) \rightarrow$ Set $^{\mathcal{C}^{o p}}$ possui adjunto à esquerda $a: \operatorname{Sets}^{\mathcal{C}^{o p}} \rightarrow \operatorname{Sh}(\mathcal{C}, J)$

\section{Demonstração:}

Fazemos $a: \operatorname{Sets}^{\mathcal{C}^{o p}} \rightarrow \operatorname{Sh}(\mathcal{C}, J)$ com $a(P)=\left(P^{+}\right)^{+}$. Pela discussão que fizemos nas etapas temos que a está bem definido pois a Proposição 2.2.21, nos garante que $P^{+}$é pré-feixe separável, logo, pela Proposição 2.2.22, $\left(P^{+}\right)^{+}=a(P)$, é feixe. Então $a$ é de fato um funtor que leva pré-feixes em feixes. 
Mostraremos a adjunção exibindo sua respectiva unidade, a qual fornece um identidade triangular do tipo $a \longrightarrow a \circ i \circ a \longrightarrow a$. Isso é bem conhecido, mas indicamos [ML98] (Teorema 1, Capítulo IV) para mais detalhes.

Agora considere $\eta_{P}: P \rightarrow P^{+}$e $\eta_{P^{+}}: P^{+} \rightarrow P^{++}$. A composição $\eta_{P^{+}} \circ \eta_{P}: P \rightarrow P^{++}$ leva um pré-feixe $P$ de $\operatorname{Sets}^{\mathcal{C}^{o p}}$ em um feixe $P^{++}$de $S h(\mathcal{C}, J)$.

Aplicando a Proposição 2.2.21 obtemos um diagrama comutativo

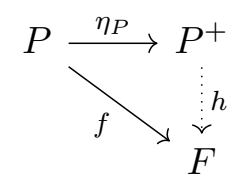

Mas podemos aplicar essa proposição uma segunda vez, agora considerando a composição $\eta_{P^{+}} \circ \eta_{P}$

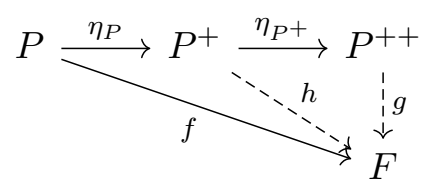

Então $f$ se fatora unicamente ao longo de $\eta_{P^{+}} \circ \eta_{P}$. Como $i(F)=F$ e $P^{++}=i(a(P))$, temos que $a$ é funtor adjunto à esquerda de $i$ e $\eta_{P^{+}} \circ \eta_{P}$ é a unidade da adjunção.

Como consequência imediata desse teorema temos:

Corolário 2.2.24 A composição a $i: S h(\mathcal{C}, J) \rightarrow S h(\mathcal{C}, J)$ é naturalmente isomorfa ao funtor identidade de $\operatorname{Sh}(\mathcal{C}, J)$

\section{Demonstração:}

Aplicando duas vezes a Proposição e 2.2.22 e depois a Proposição 2.2.19(ii), temos que se $P$ for feixe então $P^{+}$e $P^{++}$são feixes. Além disso, $\eta_{P}$ e $\eta_{P}^{+}$são isomorfismos, logo, $a(i(P))=P$ é composta de isomorfismos naturais.

Proposição 2.2.25 O funtor $a:$ Sets $^{\mathcal{C}^{o p}} \rightarrow \operatorname{Sh}(\mathcal{C}, J)$ comuta com limites finitos.

\section{Demonstração:}

Aqui vamos usar um lema bastante conhecido, sem demontrá-lo. Deixamos como referência [ML98]

Lema 2.2.26 Em Sets, limites finitos comutam com colimites filtrantes. ${ }^{2}$

Verificaremos que $P \mapsto P^{+}$comuta com limites finitos.

Seja $C$ objeto de $\mathcal{C}$ e $R \in J(C)$. Definimos um funtor $G: S e t s^{\mathcal{C}^{o p}} \rightarrow$ Sets dado por $G(P)=C_{C o m p}(R, P)$. Note que $G$ preserva limites: se $P$ é pré-feixe temos um isomorfismo natural $\operatorname{Comp}_{C}(R, P) \cong \operatorname{Hom}_{\operatorname{Set} s^{\mathcal{c}}}(R, P)$. Como o funtor $\operatorname{Hom}(R,-)$ preserva limites, temos que $G$ preserva limites.

Então, dada uma categoria de índices $I$, para todo $P: I \rightarrow S e t s^{\mathcal{C}^{o p}}$ temos:

$$
\operatorname{Comp}_{C}\left(R, \lim _{\longleftarrow} P_{i}\right) \cong \lim _{\longleftarrow} \operatorname{Comp}_{C}\left(R, P_{i}\right)
$$

Mas $P^{+}=\underset{R \in J(C)}{\lim _{\vec{f}(C)}} \operatorname{Comp}(R, P), P^{+}(C)$ é colimite de $C o m p$ sobre todas as coberturas de $C$, ordenados por inclusão reversa. Observe que dadas duas coberturas, existe um refinamento em comum dado pela interseção, pelo Exercício 2.2.7(b). Além disso, se $S \supseteq R$ e $R \supseteq T$, temos $S \supseteq T$. Com essas observações podemos concluir que Comp forma uma categoria filtrante, $\operatorname{logo} P^{+}(C)$ é colimite filtrante. Pelo Lema 2.2.26, $P \mapsto P^{+}$comuta com limites finitos. Portanto, o funtor a também comuta com limites finitos.

\footnotetext{
${ }^{2}$ Uma categoria é dita filtrante se todo diagrama finito possui cocone.
} 


\subsection{Topos Elementares}

Agora vamos definir o que é um topos, também conhecido por topos elementar. Assim como categorias abelianas, topos são categorias abstratas no sentido que não dizemos quem são os objetos ou os morfismos, apenas atribuimos certas propriedade que a categoria deve satisfazer para ser um topos. Nessa seção, além de [MLM94] usamos bastante [Joh14].

Definição 2.3.1 Seja $\mathcal{E}$ uma categoria localmente pequena com todos limites finitos e 1 seu objeto terminal. Um classificador de subobjeto em $\mathcal{E}$ consiste de um objeto $\Omega$ e um morfismo $t: 1 \rightarrow \Omega$ satisfazendo a seguinte propriedade:

Dado qualquer objeto $E$ e qualquer subobjeto $r: U \longmapsto E$, existe um único morfismo $\chi_{r}: E \longrightarrow \Omega$ que torna o diagrama abaixo um pullback

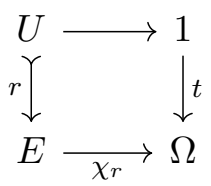

Temos que $\Omega$ é conhecido como objeto dos valores verdade, $\chi_{r}$ é morfismo classificador (ou morfismo característico) de $r$ e $t$ é o morfismo verdade.

Note que a condição de ser pullback nos dá que o classificador de subobjeto é único a menos de isomorfismo. Denotamos por $S u b_{\mathcal{E}}(E)$ o conjunto de todos os subojetos de $E$ em $\mathcal{E}$.

Pode não parecer mas qualquer estudante de matemática já se deparou com o classificador de subobjeto antes.

Exemplo 2.3.2 Seja $\mathcal{E}=$ Sets, $\Omega=\{0,1\}$ e $t: 1 \rightarrow\{0,1\}$. Tome $E$ um objeto de $\mathcal{E}$, ou seja, um conjunto. Sabemos que todo subconjunto $U$ de $E$ possui uma única função característica $\chi_{U}: E \rightarrow\{0,1\}$. Essa propriedade é exatamente a condição de ser classificador de subobjeto.

O classificador de subojeto nos fornece um isomorfismo que será bastante utilizado adiante.

Proposição 2.3.3 Uma categoria localmente pequena $\mathcal{E}$ com limites finitos possui classificador de subobjeto se, e somente se, existe um objeto $\Omega$ e um isomofismo natural $\eta$ onde

$$
\eta_{X}: S u b_{\mathcal{E}}(X) \cong \operatorname{Hom}_{\mathcal{E}}(X, \Omega)
$$

para todo $X$ objeto de $\mathcal{E}$

\section{Demonstração:}

$(\Longrightarrow)$ Suponha que $\mathcal{E}$ possui classificador de subobjeto então $\eta_{X}$ leva classes de equivalência de cada monomorfismo $r: U \longmapsto X$ em seu respectivo morfismo característico $\chi_{r}$, o qual é único, ou seja, dados dois morfismos representantes de um mesmo subobjeto, ambos possuem mesmo morfismo característico, logo, $\eta_{X}$ é injetora.

$\eta_{X}$ é sobejetora pois todo $t: 1 \longmapsto \Omega \in \operatorname{Hom}_{\mathcal{E}}(X, \Omega)$ é mono e pullback de mono é mono, então os subobjetos são unicamente determinados pelo pullback $t$ ao longo de $\chi_{r}$. Portanto, $\eta_{X}$ é uma bijeção para cada $X$. Falta checar a naturalidade.

Podemos fazer o pullback do mono $r: U \longmapsto X$, isto é, de um $S u b(X)$, ao longo de um morfismo $f: Y \rightarrow X$. Colocando esse pullback ao lado do que já tinhamos obtemos o seguinte diagrama, onde $S u b_{\mathcal{E}}(f)([r: U \longmapsto X])=\left[r^{\prime}: P \longmapsto Y\right]$ 


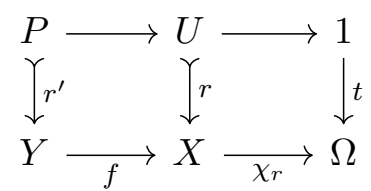

Pelo Lema dos Pullback (Lema 4.0.2), o retângulo é também um pullback. Então o pullback de $f$ em $\operatorname{Sub}(X)$ corresponde com a composição com $f$ em $\operatorname{Hom}(-, \Omega)$. Como $\mathcal{E}$ é localmente pequeno, garantimos que estamos em Sets e, portanto, vale a naturalidade.

$(\Longleftarrow)$ Suponha que $\eta_{X}: \operatorname{Sub}_{\mathcal{E}}(X) \cong \operatorname{Hom}_{\mathcal{E}}(X, \Omega)$ é isomorfismo natural para todo $X$. Pela bijeção de $\eta_{X}, S u b_{\mathcal{E}}: \mathcal{E}^{o p} \rightarrow$ Sets é representável (Definição 2.2.1) e existe um único subobjeto de $\Omega$, digamos, $t^{\prime}: T \longmapsto \Omega$, que é identificado, por $\eta_{X}$ com a identidade id $: \Omega \rightarrow \Omega$ em $\operatorname{Hom}(\Omega, \Omega)$.

Além disso, subojetos $[U \longmapsto X]$ de $X$ correspondem a um único morfismo $\chi: X \rightarrow \Omega$. Como $\eta_{X}$ é natural obtemos

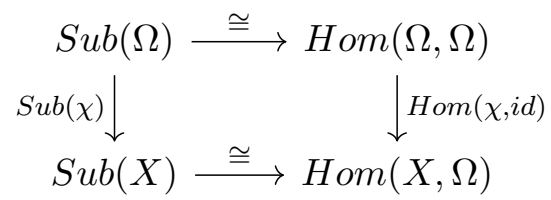

cujas aplicações fornecem um diagrama comutativo do tipo

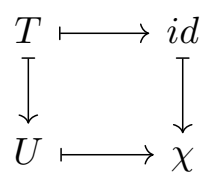

Ou seja, $(S u b(\chi))(T)=U$. Então $U$ é o pullback de $T$ ao longo de $\chi$, isto é, o seguinte diagrama é um pullback:

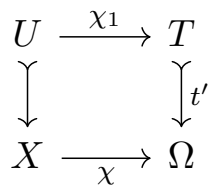

O diagrama da definição de classificador de subobjetos já temos, resta apenas verificar que $T$ é terminal em $\mathcal{E}$. Podemos trocar $U$ por $X$ de forma que $U \longmapsto X$ seja a identidade $X \rightarrow X$.

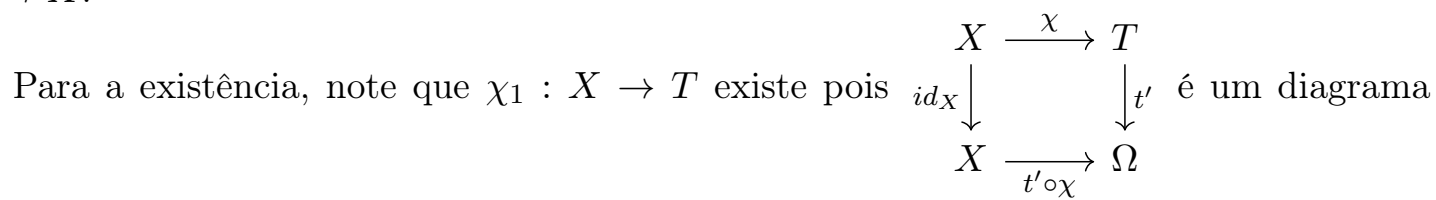
de pullback. É claro que comuta, para ver que é de pullback considere outro diagrama

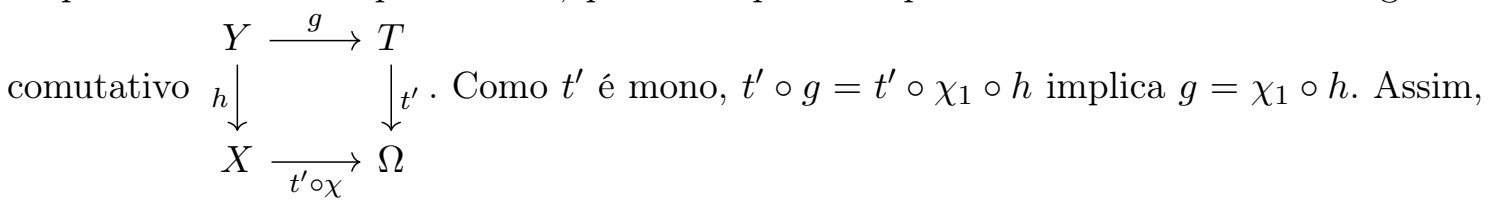
$h: Y \rightarrow X$ é o único morfismo tal que $i d_{X} \circ h=h$ e $g=\chi_{1} \circ h$, assim as condições de pullback estão satisfeitas.

Para a unicidade, suponha que exista outro morfismo $\chi_{2}: X \rightarrow T$. Então:

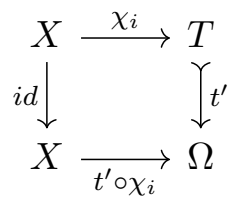


com $i=1$ ou 2 é um pullback pois $t^{\prime}$ é mono. Mas recorde que o morfismo $\chi$ que forma esse pullback é único, logo, $t^{\prime} \circ \chi_{1}=\chi=t^{\prime} \circ \chi_{2}$. Usando mais uma vez que $t^{\prime}$ é mono concluímos que $\chi_{1}=\chi_{2}$.

Isso significa que o mapa $X \rightarrow T$ é único para cada objeto $X$ em $\mathcal{E}$, isto é, $T$ é terminal.

Vamos agora introduzir um conceito que generaliza a noção de conjunto de funções.

Definição 2.3.4 Seja $\mathcal{E}$ uma categoria com todos os produtos binários, $B$ e $C$ objetos de $\mathcal{E}$. Um objeto exponencial consiste de um objeto $C^{B}$ e um morfismo ev : $C^{B} \times B \rightarrow C$ satisfazendo a seguinte propriedade:

Dado qualquer objeto $A$ e qualquer morfismo $f: A \times B \rightarrow C$ existe um único morfismo $\tilde{f}: A \rightarrow C^{B}$ tal que $e v \circ\left(\widetilde{f} \times i d_{B}\right)=f$. Em termos dos diagramas:

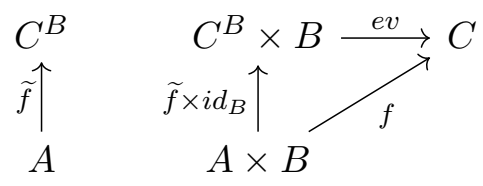

Dizemos que $e v$ é o mapa de avaliação e $\widetilde{f}$ é a transposta exponencial de $f$.

Por outro lado, se tivermos $g: A \rightarrow C^{B}$, escrevemos $\bar{g}=e v \circ\left(g \times i d_{B}\right): A \times B \rightarrow C$ e chamamos $\bar{g}$ de transposta de $g$. Pela unicidade da transposta exponencial, obtemos $\widetilde{\bar{g}}=g$. Mais ainda, também temos $\widetilde{\widetilde{f}}=f$ para toda $f: A \times B \rightarrow C$.

Definição 2.3.5 Dizemos que uma categoria é cartesianamente fechada se tiver produtos binários e se cada objeto $B$ e $C$ admitir objeto exponencial $C^{B}$.

Note que nesse caso para cada objeto $B$ podemos definir um funtor $(-)^{B}: \mathcal{E} \rightarrow \mathcal{E}$ e esse funtor é adjunto à direita do funtor $(-) \times B$. Assim, por propriedade da adjunção, temos um isomorfismo $\operatorname{Hom}_{\mathcal{E}}(B \times A, C) \cong \operatorname{Hom}_{\mathcal{E}}\left(A, C^{B}\right)$.

Finalmente vamos definir os topos elementares, os quais chamaremos apenas de topos.

Definição 2.3.6 Um topos é uma categoria $\mathcal{E}$ tal que:

1. $\mathcal{E}$ possui todos os limites finitos;

2. $\mathcal{E}$ possui classificador de subojeto.

3. $\mathcal{E}$ é cartesianamente fechada.

Observação 2.3.7 Ao longo do trabalho usaremos colimites finitos dos topos, não faremos detalhes da construção dos mesmo mas a ideia para mostrar a existência é definir um funtor $P: \mathcal{E}^{o p} \rightarrow \mathcal{E}$ por $P(B)=\Omega^{B}$ e, dado morfismo $f: B \rightarrow C, P(f): \Omega^{C} \rightarrow \Omega^{B}$ é a composta $e v \circ(1 \times f)$. Tal funtor é monádico, desse fato segue que $\mathcal{E}^{o p}$ possui limites finitos, os quais são colimites em $\mathcal{E}$. Ver Capítulo IV, Seção 5 de [MLM94].

Exemplo 2.3.8 1. Sets: De fato, sabe-se que a categoria dos conjuntos possui pullbacks e objeto terminal - à saber, respectivamente, um subconjunto do produto cartesiano de dois conjutos e o conjunto unitário - portanto, possui todos os limites finitos. Pelo exemplo 1.3.2, possui classificador de subojeto. A exponencial em Sets também é bem conhecida: sejam $X$ e $Y$ conjuntos, temos $Y^{X}=\{g: X \rightarrow Y\}$ e o morfismo $e v$ é o mapa de valoração que leva cada para $(g, x) \in Y^{X} \times X$ para $g(x) \in Y$. Assim, para qualquer morfismo $h: Z \times X \rightarrow Y$ existe um único morfismo $\widetilde{h}: Z \rightarrow Y^{X}$ tal que $\widetilde{h}(z)(x)=h(z, y)$. 
2. $S e t s^{\mathcal{C o p}^{\circ}}$, com $\mathcal{C}$ uma categoria pequena: O terminal é o pré-feixe constante denotado por 1 tal que $1(A)=\{*\}$ para todo objeto $A$ de $\mathcal{C}$, onde $\{*\}$ é o conjunto unitário, que é terminal em Sets. Como pré-feixes são calculados ponto a ponto também não é difícil verificar que o pullback existe. Com isso temos que Sets ${ }^{\mathcal{C}^{o p}}$ possui limites finitos, agora vamos descrever o classificador de subobjeto. Pelo Lema de Yoneda, dado $A$ em $\mathcal{C}, \Omega(A) \cong \operatorname{Hom}(y(A), \Omega)$ é um isomorfismo, logo, existe uma correspondência bijetora entre $\Omega(A)$ e morfismos $\operatorname{Hom}(-, A) \rightarrow \Omega$, então corresponde a uma classe de isomorfismos de subobjetos de $\operatorname{Hom}(-, A)$. Seja $F$ subfuntor de $\operatorname{Hom}(-, A)$, determinado por $R$ crivo de $A$, definimos $\Omega(A)=\{R \mid R$ é crivo de $A\}$ e $\Omega(f)(R)=\{g \mid \operatorname{codom}(g)=A, f \circ g \in R\}$, para cada $f: A \rightarrow B$ e $R \in \Omega(A)$.

Assim $\Omega$ é um funtor em Sets $^{\mathcal{C}^{o p}}$.

Definimos o morfismo verdade $t_{A}$ como o crivo maximal, que é formado por todos os morfismos com codomínio $A$ e, dessa forma, $t_{A}: 1 \rightarrow \Omega$ é uma transformação natural.

Seja $F^{\prime} \longmapsto F$ subobjeto em $S e t s^{\mathcal{C}^{o p}}$, para cada $x \in F(A)$ definimos uma transformação natural $\phi_{A}(x)=\left\{f \mid \operatorname{codom}(f)=A, F(f)(x) \in F^{\prime}(\operatorname{dom} A)\right\}$.

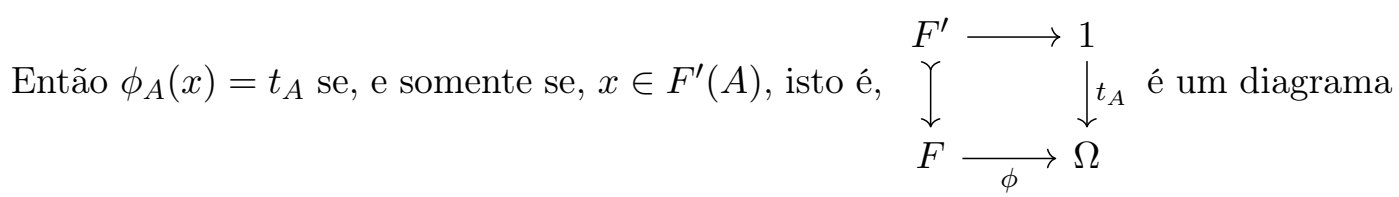
de pullback. Falta verificar a unicidade de $\phi$.

Observe que dado crivo $R, f \in R$ se, e somente se, $\Omega(f)(R)=t_{d o m(f)}$. Se $\psi$ é uma outra transformação natural que fazer do diagrama acima um pullback, temos $f \in \psi_{\operatorname{codom}(f)}(x)$ se, e só se, $\psi_{\operatorname{dom}(f)}(F(f)(x))=t_{d o m_{f}}$, se e só se, $F(f) \in F^{\prime}(\operatorname{dom}(f))$, então $\phi=\psi$.

Assim concluímos que o funtor $\Omega$ é classificador de subobjeto em $\operatorname{Sets}^{\mathcal{C}^{o p}}$.

Para verificar a existência do objetos exponencial mais uma vez usamos o Lema de Yoneda. Dados $F, G$ em $\operatorname{Sets}^{\mathcal{C}^{o p}}$, usamos a corresponência bijetora entre $G^{F}(A)$ e morfismos $\operatorname{Hom}(-, A) \rightarrow G^{F}$, que por sua vez estão em correspondência com $H o m(-, A) \times F \rightarrow G$ para definir $G^{F}(A)=H o m(-, A) \times F \rightarrow G$, para todo $A$ em $\mathcal{C}$ e $G^{F}(f)=\operatorname{Hom}(-, f) \times i d: H o m(-, B) \times F \rightarrow H o m(-, A) \times F, h \mapsto h \circ f$, para $f: A \rightarrow B$ em $\mathcal{C}$.

Seja $\psi: \operatorname{Hom}(-, A) \times G \rightarrow G$ e $x \in F(A)$, definimos o morfismo avaliação $e v$ : $G^{F} \times F \rightarrow G$ por $e v_{A}(\psi, x)=\psi_{A}\left(i d_{A}, x\right)$ e para todo $g: H \times F \rightarrow G$ definimos a exponencial transposta $\bar{g}: H \rightarrow G^{F}$ por $\left(\bar{g}_{A}(z)\right)_{B}(f, x)=g_{B}(H(f)(z), x)$, onde $z \in H(A), f: A \rightarrow B$ e $x \in F(B)$.

Sabendo isso verifica-se que $\underset{H \times F}{G^{F} \times F \underset{g}{\stackrel{e v}{\longrightarrow}} G}$ e temos que $G^{F}$ é exponencial em $\operatorname{Sets}{ }^{\mathcal{C}^{o p}}$

3. Topos de Grothendieck: Vamos apenas esboçar a verificação. Vimos no Teorema 2.2.23 um par de funtores adjuntos entre $\operatorname{Sets}^{\mathcal{C}^{\circ p}}$ e $S h(\mathcal{C}, J)$. Em 2.2.25 vimos ainda que o funtor feixe associado $a: \operatorname{Sets}^{\mathcal{C}^{o p}} \rightarrow \operatorname{Sh}(\mathcal{C}, J$ preserva limites finitos. Como $S e t s^{\mathcal{C}^{o p}}$ possui todos os limites finitos, temo que $S h(\mathcal{C}, J)$ também possui.

Para $S e t S^{\mathcal{C}^{o p}}$ definimos o morfismo característico através do mergulho de Yoneda $y(A)$, para $S h(\mathcal{C}, J)$ o procedimento é análogo mas agora define-se $\Omega$ como o conjunto de subobjetos de $a(y(A))$, onde $a$ é o funtor feixe associado introduzido na seção 2.2. 
O objeto exponencial em $S h(\mathcal{C}, J)$ é definido da mesma forma como em $S e t s^{\mathcal{C}^{o p}}$, pois se $G$ é um feixe para uma topologia de Grothendieck $J$ em $\mathcal{C}$, então o pré-feixe $G^{F}$ é feixe para qualquer $F$ e assim $G^{F}$ é exponencial em $S h(\mathcal{C}, J)$.

Agora que finalmente definimos um topos, vale uma observação sobre o uso da palavra no plural. Segundo uma nota de rodapé de [Mcl90], a palavra topos é uma de origem francesa que vem de "topologie" (topologia). Por isso, A. Grothendieck usa o pural obedecendo as regras do francês, onde não se modifica palavras terminadas com "s". O uso de "topoi" é comum entre os que entendem o termo como sendo de origem grega e "toposes" se justifica pela regra da lingua inglesa. Seguiremos a regra da lingua portuguesa, a qual coincide com a francesa, e sempre teremos "topos", seja no plural ou no singular.

Definição 2.3.9 Sejam $\mathcal{E}$ e $\mathcal{E}^{\prime}$ dois topos. Chamamos de funtor lógico um funtor $T$ : $\mathcal{E} \rightarrow \mathcal{E}^{\prime}$ que preserva limites finito, exponenciais e classificadores de subobjetos. Em outras palavras, preserva a estrtura de topos.

Isso significa que se $X \stackrel{p}{\leftarrow} X \times Y \stackrel{q}{\rightarrow} Y$ são projeções de um produto em $\mathcal{E}$, então temos projeções de um produto $T(X) \stackrel{T(p)}{\longleftarrow} T(X \times Y) \stackrel{T(q)}{\longrightarrow} T(Y)$ em $\mathcal{E}^{\prime}$; se $E \stackrel{e}{\longrightarrow} X \underset{f^{\prime}}{\stackrel{f}{\longrightarrow}} Y$ é diagrama de equalizador em $\mathcal{E}$ então $T(E) \stackrel{T(e)}{\longrightarrow} T(X) \underset{T\left(f^{\prime}\right)}{\stackrel{T(f)}{\longrightarrow}} T(Y)$ também é equalizador em $\mathcal{E}^{\prime}$; se $t: 1 \rightarrow \Omega$ é classificador de subobjeto em $\mathcal{E}$, então $T(t): T 1 \rightarrow T(\Omega)$ é classificador de subobjeto em $\mathcal{E}^{\prime}$ e se $C^{B}$ é exponencial em $\mathcal{E}$, então $T\left(C^{B}\right)$ é exponencial em $\mathcal{E}^{\prime}$.

As projeções do tipo $\mathcal{E}_{1} \times \mathcal{E}_{2} \stackrel{\pi_{i}}{\longrightarrow} \mathcal{E}_{i}$ e o mapa inclusão $\operatorname{Sets}_{f} \rightarrow$ Sets, onde Sets $s_{f}$ é a categoria dos conjuntos finitos, são alguns exemplos de funtor lógico.

Agora vamos mostrar diversar propriedades dos topos, muitas delas também vimos para categorias abelianas. No Capítulo 4 disponibilizamos uma tabela listando os resultados que apresentamos ao longo da Dissertação e são compartilhados por ambas as categorias.

Proposição 2.3.10 Em um topos, todo monomorfismo é um equalizador.

\section{Demonstração:}

Seja $m: X \rightarrow Y$ um monomorfismo. Considere $t_{Y}=t o !_{Y}$, onde $!_{Y}$ é a única flecha que vai de $Y$ no objeto terminal 1. Sendo assim, e por definição de classificador de subobjeto, o diagrama abaixo comuta

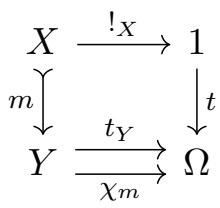

Seja $f: W \rightarrow Y$ um morfismo que equaliza $t_{Y}$ e $t$, temos $\chi_{m} \circ f=t_{Y} \circ f$ e $t_{Y} \circ f=$ $\left(t \circ !_{Y}\right) \circ f=t \circ\left(!_{Y} \circ f\right)=t \circ !_{W}$, onde a última igualdade vale por cauda da propriedade universal do objeto terminal.

Então

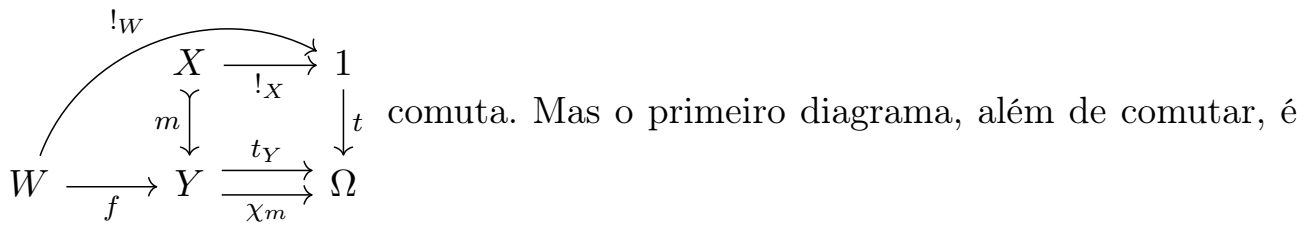

um pullback (por definição de classificador de subobjeto). Então conseguimos um morfismo $g: W \rightarrow X$ tal que $m \circ g=f$ e, como $m$ é mono, $g$ é unica.

Desta forma provamos que o par $(X, m)$ é o equalizador de $t_{Y}$ e $\chi_{m}$.

Corolário 2.3.11 Todo morfismo em um topos que é, simultaneamente, epi e mono, é um isomorfismo. 


\section{Demonstração:}

Vimos acima que todo mono em um topos é um equalizador, mas, para qualquer categoria, um equalizador que é epi é um isomorfismo.

Para prosseguir precisamos dizer o que é uma relação de equivalência numa categoria com liminites finitos.

Definição 2.3.12 Seja $\mathcal{E}$ uma categoria com limites finitos, $R \underset{b}{\stackrel{a}{\longrightarrow}} X$. um par paralelo de morfismo de $\mathcal{E}$. Dizemos que $(a, b)$ é uma relação de equivalência em $X$ se:

1. $R \stackrel{(a, b)}{\longrightarrow} X \times X$ é monomorfismo;

2. O subobjeto diagonal $X \stackrel{\Delta}{\longrightarrow} X \times X$ se fatora por $(a, b)$, em outras palavras, existe $X \stackrel{c}{\longrightarrow} R$ tal que $a c=b c=i d_{X}$;

3. Existe um monomorfismo $R \stackrel{\tau}{\longrightarrow} R$ tal que $b \tau=a$ e $a \tau=b$;

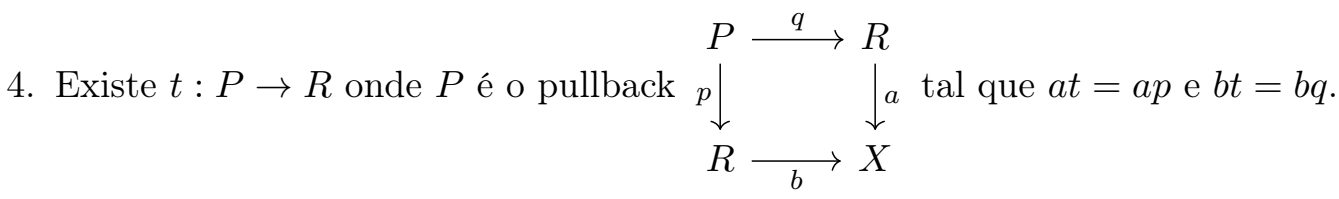

O item 1 define relação, o 2 relação reflexiva, o 3 relação simétrica, o 4 relação transitiva.

Definição 2.3.13 Seja $f: X \rightarrow Y$ um morfismo dizemos que o par de flechas $(a, b): R \rightarrow$ $X$ é kernelpar de $f$ se é o pullback de $f$ ao longo do próprio $f$, ou seja, o diagrama abaixo é um pullback

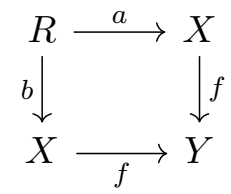

Todo kernelpar determina uma relação de equivalência e uma relação de equivalência é dita efetiva se é um kernelpar.

Proposição 2.3.14 Em um topos toda relação de equivalência é efetiva.

\section{Demonstração:}

Não exibiremos uma prova para esse resultado aqui pois o enunciamos mais para introduzir a noção de relação de equivalência efetiva, que torna a aparecer no enunciado do Teorema de Giraud. Uma referência é [Joh14], Proposição 1.23.

Definição 2.3.15 Seja $X$ um objeto de um topos, o singleton de $X$ é um morfismo \{\}$_{X}: X \rightarrow \Omega^{X}$ que corresponde à transposta exponencial de $\delta: X \times X \rightarrow \Omega$, onde $\delta$ é morfismo característico da diagonal $\triangle: X \rightarrow X \times X$.

Definição 2.3.16 Um morfismo parcial $f: X \rightarrow Y$ em um topos é um diagrama da forma

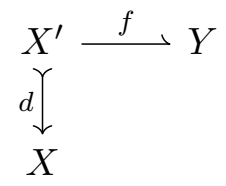


Definição 2.3.17 Um morfismo parcial com codomínio $Y$ é dito representável se existe um monomorfismo $\eta: Y \rightarrow \widetilde{Y}$ tal que para qualquer $f: X \rightarrow Y$ existe um único $\widetilde{f}: X \rightarrow \widetilde{Y}$ que torna o seguinte diagrama um pullback

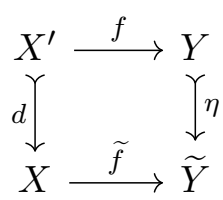

Note que se $Y=1$ na definição de morfismo parcial representável, então caímos na definição de classificador de subobjeto.

O Teorema a seguir já foi um axioma para definir topos elementar, mas foi retirado quando W. Lawvere e M. Tierney perceberam que poderia ser obtido como consequência da existência do classificador de subobjeto em uma categoria cartesianamente fechada.

Teorema 2.3.18 Em um topos todos os mapas parciais são representáveis.

\section{Demonstração:}

Seja \{\}$: Y \rightarrow \Omega^{Y}$ o morfismo singleton. Considere o seu gráfico graf $(\{\})=\left(\{\}, i d_{Y}\right)$ : $Y \rightarrow \Omega^{Y} \times Y$ e $\chi$ a respectiva função caracterísca de $\operatorname{graf}(\{\})$, que é uma seção e, portanto, um mono. Então temos o seguinte diagrama de pullback

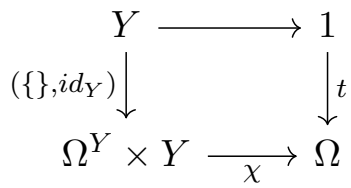

Tome $\tilde{\chi}: \Omega^{Y} \rightarrow \Omega^{Y}$ a transposta exponencial de $\chi$ e defina $\tilde{Y} \longmapsto \Omega^{Y}$ como o equalizador de $\tilde{\chi}$ e $i d_{Y}$, isto é, $\tilde{Y} \succ \Omega^{Y} \underset{i d_{\Omega^{Y}}}{\stackrel{\tilde{\chi}}{\longrightarrow}} \Omega^{Y}$

Uma vez que \{\} é um monomorfismo, temos que o diagrama comutativo a seguir é um pullback

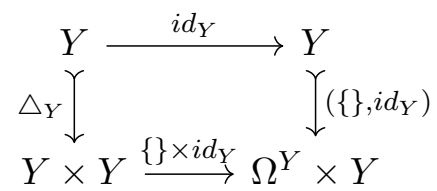

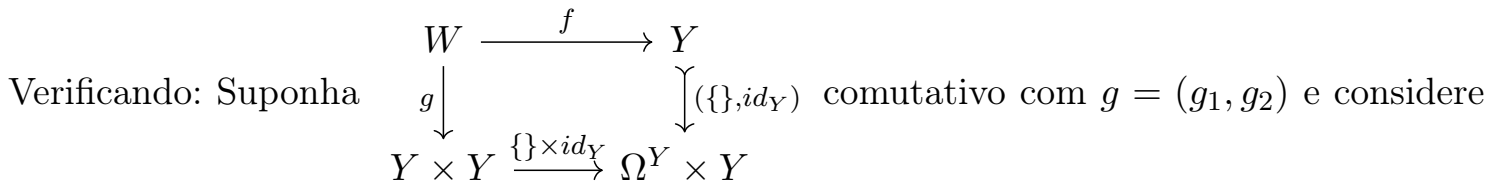
as projeções $p_{y}: \Omega^{Y} \times Y \rightarrow Y$ e $p_{\Omega^{Y}}: \Omega^{Y} \times Y \rightarrow \Omega^{Y}$.

Veja que $p_{\Omega^{Y}} \circ\{\} \circ g_{1}=p_{\Omega^{Y}} \circ\{\} \circ f$ e $p_{Y} \circ\{\} \circ g_{2}=p_{Y} \circ\{\} \circ f$, então $g=(f, f)$ e encontramos $h: W \rightarrow Y$ tal que $i d_{Y} \circ h=f$ e $\triangle_{Y} \circ h=g$, agora basta fazer $h=f$.

Com isso temos dois quadrados de pullback um ao lado do outro

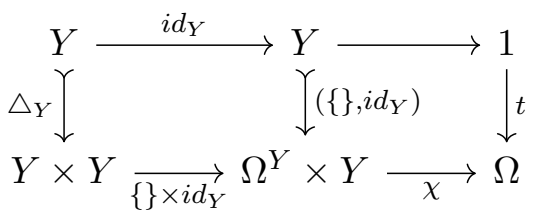

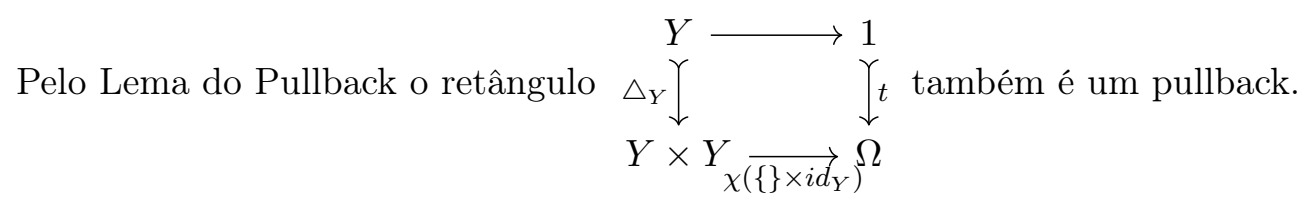


Isso significa que $\chi\left(\{\} \times i d_{Y}\right)$ classifica $\triangle_{Y}, \operatorname{logo}, \tilde{\chi}_{Y} \circ\{\}=\{\}$. Sendo assim, \{\} se fatora em $\widetilde{Y} \longmapsto \Omega^{Y}$, que é o equalizador de $\tilde{\chi}$ e $i d_{\Omega^{Y}}$. Então existe um único monomorfismo $\eta: Y \rightarrow \widetilde{Y}$.

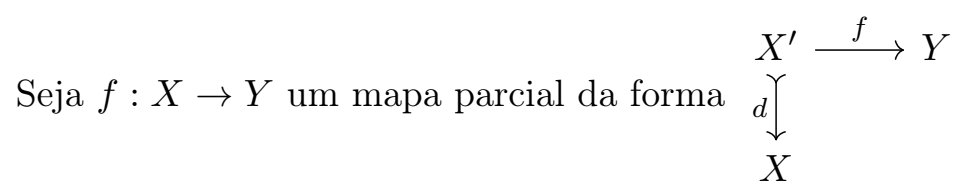

Considere o mapa $(d, f): X^{\prime} \longmapsto X \times Y$ (que é mono pois $d$ é mono) e seu respectivo classificador $\psi: X \times Y \rightarrow \Omega$. Vamos mostrar que $\widetilde{\psi}: X \rightarrow \Omega^{Y}$ se fatora em $\tilde{Y}$, ou seja, que o diagrama abaixo é um pullback.

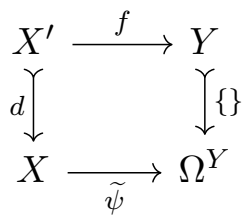

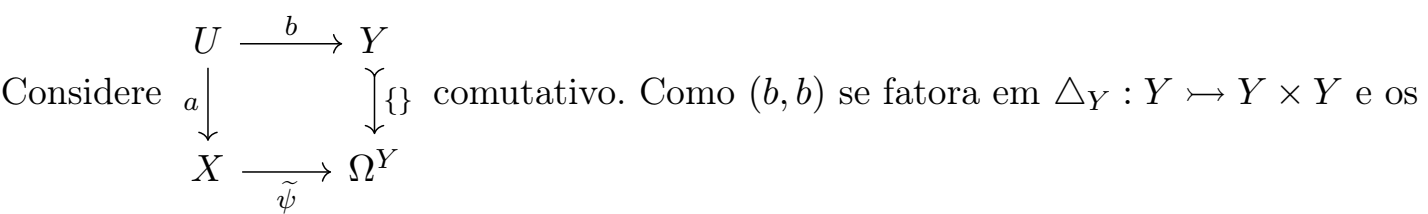
dois diagramas seguintes comutam, obtemos $\psi(a, b)=\phi(b, b)$
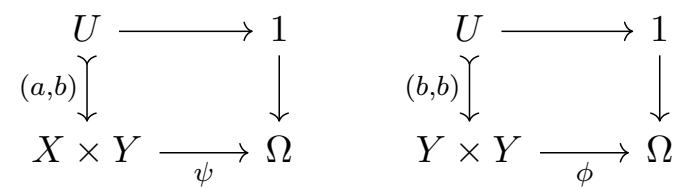

Então $(a, b)$ se fatora de forma única em $(d, f): X^{\prime} \longmapsto X \times Y$ e, de fato, $\left(\Omega^{Y}, \widetilde{\psi},\{\}\right)$ é um pullback. Agora que temos uma fatoração de $\widetilde{\psi}$, vamos chamar de $\widetilde{f}$ o morfismo que vai de $X$ em $\widetilde{Y}$ (nesse caso não estamos falando da transposta exponencial da $f$ ). Assim:

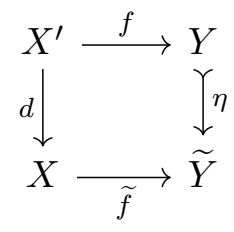

comuta.

Resta ver que $\tilde{f}$ é único. Suponha que existam $f_{1}$ e $f_{2}$ satisfazendo o mesmo que $\widetilde{f}$. Vimos que as exponenciais transpostas da composição de tipo $X \stackrel{f_{i}}{\longrightarrow} \widetilde{Y} \longmapsto \Omega^{Y}$ classificam o mesmo subobjeto $(d, f): X^{\prime} \longmapsto X \times Y$ de $X \times Y$, então

$$
X \stackrel{f_{1}}{\longrightarrow} \tilde{Y} \longmapsto \Omega^{Y}=X \stackrel{f_{2}}{\longrightarrow} \widetilde{Y} \longmapsto \Omega^{Y}
$$

Como $\tilde{Y} \longmapsto \Omega^{Y}$ é mono, $f_{1}=f_{2}$.

A partir de um mapa parcial $f$ qualquer encontramos um único $\widetilde{f}$ que torna $f$ representável.

Temos uma consequência que já se relaciona com um conceito introduzido no capítulo de Álgebra Homológica.

Corolário 2.3.19 Os objetos $\tilde{Y}$ construídos no teorema anterior são injetivos. Portanto, um topos possui suficientes injetivos. 


\section{Demonstração:}

Possuir suficientes injetivos significa que para objeto $X$ no topos, existe um monomorfismo $X \longmapsto E$, onde $E$ é injetivo. Nosso $E$ é $\widetilde{Y}$, devemos apenas verificar que ele é injetivo.

Dado um diagrama da forma ${ }_{\widetilde{Y}}^{I^{\prime}}{ }^{X}$ vamos mostrar que ele comuta, como pede a definição de objeto injetivo.

O triângulo acima forma o pullback $\stackrel{X^{\prime \prime}}{\downarrow} \underset{Y}{\downarrow} \underset{\eta}{\downarrow} \underset{\widetilde{Y}}{\downarrow}$

Pelo Teorema 2.3.18, existe um único $X \rightarrow \widetilde{Y}$ que faz do próximo diagrama também um pullback

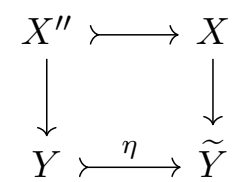

Mas os dois pullbacks representam o mesmo mapa parcial $X \rightarrow Y$, logo, o único $X \rightarrow \widetilde{Y}$ faz o diagrama inicial comutar.

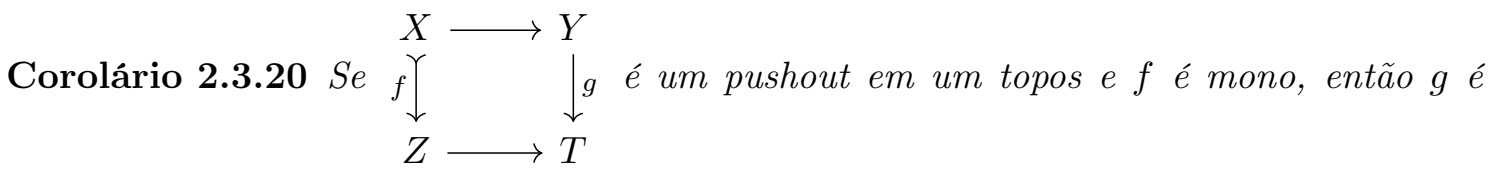
mono e esse pushout é também um pullback.

\section{Demonstração:}

Seja $h: Z \rightarrow \widetilde{Y}$ o mapa parcial da forma $\underset{Z}{X}{ }_{Z} \succ Y$.

Como todo mapa parcial em um topos é representável, temos o pullback

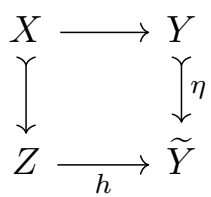

Temos que o cone $(\eta, h)$ se fatora ao longo de $T$. Em particular, o monomorfismo $\eta$ se fatora ao longo de $g$, portanto, $g$ é mono. Além disso, sendo o diagrama acima um pullback, o diagrama originalmente enunciado também é.

Vamos falar um pouco de fatoração pela imagem, isto é, que todo morfismo se fatora em epi e mono assim como acontece em Sets e em categorias abelianas.

Definição 2.3.21 Seja $f$ um morfismo de um topos. Dizemos que $m$ é imagem de $f$ se:

1. $m$ é mono e $f=m \circ e$ para algum morfismo $e$.

2. se existe outro mono $m^{\prime}$ tal que $f=m^{\prime} \circ e^{\prime}$ para algum $e^{\prime}$, então $m=m^{\prime} \circ g$ para um único morfismo $g$ (pois $m$ é mono).

Em outras palavras, $m$ é o menor subobjeto em que $f$ se fatora. 
Veremos na próxima seção que sempre podemos fatorar $f$, de forma única a menos de isomorfismo, por um epi e um mono. Por essa unicidade, teremos que $f$ sempre admite fatoração pela imagem.

Agora considere um morfismo $f: A \rightarrow B$ e um subojeto de $A, s: S \longmapsto A$, se tomarmos sua fatoração pela imagem obteremos um diagrama comutativo como o abaixo:

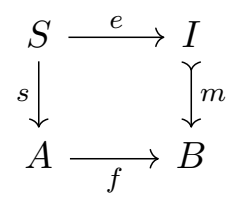

Isso nos leva a crer que existe um mapa que leva subobjetos de $A$ em subobjetos de $B$. Veremos isso em maiores detalhes na seção sobre Álgebras de Heyting em um topos, essa discussão é importante para começarmos a falar das propriedades internas de um topos, mas antes de chegarmos nisso precisamos introduzir as noções de morfismos geométricos e categoria slice.

\subsection{Morfismos Geométricos e Categoria Slice}

$\mathrm{Na}$ seção anterior definimos um tipo de morfismo entre topos elementares chamado funtor lógico, mas há outro morfismo entre topos muito útil. Agora nossas principais referências foram [MLM94] e [Joh02a].

Definição 2.4.1 Sejam $\mathcal{E}$ e $\mathcal{F}$ topos. Um morfismo geométrico $f: \mathcal{F} \rightarrow \mathcal{E}$ consiste de um par de funtores $f_{*}: \mathcal{F} \rightarrow \mathcal{E}$ (imagem direta) e $f^{*}: \mathcal{E} \rightarrow \mathcal{F}$ (imagem inversa) tais que

1. $f^{*}$ é adjunto à esquerda de $f_{*}$ e denotamos por $f^{*} \dashv f_{*}$.

2. $f^{*}$ preserva limites finitos, isto é, exato à esquerda.

Dados dois morfismos geométricos $f, g: \mathcal{F} \rightarrow \mathcal{E}$, dizemos que $\eta: f \rightarrow g$ é uma transformação geométrica se $f^{*} \rightarrow g^{*}$ é uma transformação natural (isso implica que existe uma única $f_{*} \rightarrow g_{*}$ tranformação natural, induzida pela adjunção).

Segue da primeira condição que $f^{*}$ preserva colimites pequenos e $f_{*}$ preserva limites pequenos.

Por todo o trabalho que fizemos até aqui é muito natural esperar que tenhamos exemplos de morfismo geométricos para feixes e topos de Grothendiek. Vamos descrevê-los.

Exemplo 2.4.2 Sejam $X$ e $Y$ espaços topológicos. Uma função contínua $f: X \rightarrow Y$ induz um funtor $f^{-1}: \mathcal{O}(Y) \rightarrow \mathcal{O}(X)$. Desta forma definimos a imagem direta $S h(f)_{*}=$ $f_{*}: S h(X) \rightarrow S h(Y)$ tomando funtores $\mathscr{F}$ em $S h(X)$ e compondo com $f^{-1}$, ou seja, $f_{*}(\mathscr{F})(V)=\mathscr{F}\left(f^{-1}(V)\right)$, onde $V$ é aberto de $Y$.

Para imagem inversa $S h(f)^{*}=f^{*}: S h(Y) \rightarrow S h(X)$ consideramos feixes $\mathscr{G}$ em $S h(Y)$ como homeomorfismos locais sobre $Y$, isto é, fibrados étale $p: \widetilde{\mathscr{G}} \rightarrow Y$, onde $\widetilde{\mathscr{G}}=\coprod_{y \in Y} \mathscr{F}_{y}$

(ver seção 1.1) e tomamos seu pullback com $f$

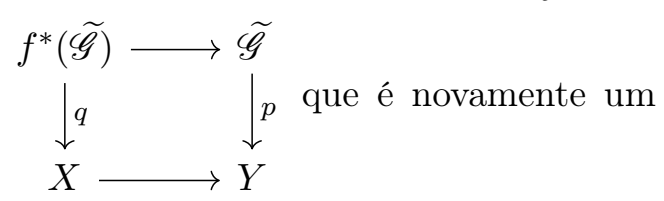

fibrado étale.

De fato, seja $(x, g) \in f^{*}(\widetilde{\mathscr{G}})=\left\{(x, g) \mid f(x)=p(g)\right.$ e $p_{\left.\right|_{U}}: U \rightarrow p(U)$ homeomorfismo . Como $p$ é étale existe $U$ vizinhança aberta de $g$ tal que $p(U)$ é aberto em $Y$. Como $f$ é contínua, obtemos $W=f^{-1}(p(U)) \times U$ vizinhança de $(x, g)$ aberta em $X \times \widetilde{\mathscr{G}}$. Fazendo a intersecção, segue que $W \cap f^{*}(\mathscr{\mathscr { G }})$ é vizinhaça de $(x, g)$ aberta em $f^{*}(\widetilde{\mathscr{G}})$. Por 
fim, por definição de pullback $q(W)=f^{-1}(p(U))$ é um aberto, $q_{\left.\right|_{W}}: W \rightarrow f^{-1}(p(U))$ é homeomorfismo, logo, $q$ é homeomorfismo local, ou seja, um fibrado étale.

Temos que $f^{*}$ preserva limites pois as fibras são dadas por colimites filtrantes e estes comutam com limites finitos (Lema 1.2.26).

Explicitamos quem são as imagens diretas e inversas do morfismo geométrico $S h(X) \rightarrow$ $S h(Y)$, deixamos [Joh14] como referência para verificar a adjunção entre esses funtores.

Exemplo 2.4.3 No caso de feixes em casais o morfismo geométrico $S h(\mathcal{C}, J) \rightarrow \operatorname{Sets}^{\mathcal{C o p}^{\circ}}$ apareceu na seção 2.2. A imagem direta é a inclusão $i: S h(\mathcal{C}, J) \rightarrow S e t s^{\mathcal{C}^{o p}}$ e a imagem inversa é o funtor feixe associado $a: \operatorname{Sets}^{\mathcal{C}^{o p}} \rightarrow \operatorname{Sh}(\mathcal{C}, J)$. Provamos a adjunção no Teorema 2.2.23 e que preserva limites finitos na Proposição 2.2.25.

No próximo capítulo usaremos a noção de morfismo geométrico sobrejetor, vamos já providenciar uma definição para isso.

Definição 2.4.4 Seja $f: \mathcal{F} \rightarrow \mathcal{E}$ um morfismo geométrico. Dizemos:

1. $f$ é uma inclusão se as seguinte condições equivalentes são satisfeitas:

a) $f_{*}$ é pleno e fiel.

b) A counidade da adjunção $\left(f^{*} \dashv f_{*}\right)$ é um isomorfismo.

2. $f$ é uma sobrejeção se as seguinte condições equivalentes são satisfeitas:

a) $f^{*}$ reflete isomorfismos, i.e., se $f^{*}(\alpha)$ é iso, então $\alpha$ é iso para todo morfismo $\alpha \in \mathcal{E}$

b) $f^{*}$ é fiel.

c) A unidade da adjunção $\left(f^{*} \dashv f_{*}\right)$ é monomorfismo.

Exemplo 2.4.5 Já vimos que $f: S h(X) \rightarrow S h(Y)$ é um morfismo geométrico. Dependendo da situação, pode ser de inclusão ou de sobrejeção.

1. Seja $f: X \rightarrow Y$ contínua e sobrejetora. Considere $\alpha, \beta: \mathscr{F} \rightarrow \mathscr{G}$ morfismos de feixes em $Y$ onde $\left(f^{*}(\mathscr{R})\right)_{x}=\mathscr{F}_{x},\left(f^{*} \alpha\right)_{x}=\alpha_{f(x)}$ e procedemos analogamente para $\mathscr{G}$ e $\beta$. Uma vez que $f$ é sobrejetora, temos que para $y \in Y, \alpha_{y}=\beta_{y}: \mathscr{F}_{y} \rightarrow \mathscr{G}_{y}$, logo, $\alpha=\beta$. Então $f^{*}$ é fiel e $f: S h(X) \rightarrow S h(Y)$ é sobrejeção.

2. Seja $f: X \rightarrow Y$ inclusão contínua. Tomes $\mathscr{F}$ um feixe em $X$. Faremos cálculos nos stalks para obter um isomorfismo. Seja $V$ aberto em $Y$ :

$$
\begin{aligned}
& {\left[f^{*} f_{*}(\mathscr{F})\right]_{x}=\left[f_{*}(\mathscr{F})\right]_{f(x)} \cong \underset{f(x) \in V}{\lim _{*}} f_{*}(\mathscr{F})(V)} \\
& \cong \lim _{f(x) \in V} \mathscr{F}\left(f^{-1}(V)\right) \quad \text { Definição de } f_{*} \\
& \cong \lim _{x \in U} \mathscr{F}(U) \quad \text { Todo aberto } U \text { de } X \text { é } \\
& \cong \mathscr{F}_{x} \\
& U=V \cap Y=f^{-1}(V)
\end{aligned}
$$

Então $f^{*} f_{*}=i d$

Agora vamos falar da categoria slice.

Definição 2.4.6 Seja $\mathcal{C}$ uma categoria e $C$ um objeto de $\mathcal{C}$. Definimos a categoria slice, denotado por $\mathcal{C} \downarrow C$, onde os objetos são os morfismos de $\mathcal{C}$ cujo codominío é $C$ e os morfismos são tais que dado dois objetos $d: D \rightarrow C$ e $e: E \rightarrow C$ de $\mathcal{C} \downarrow C$, o seguinte diagrama comuta: 


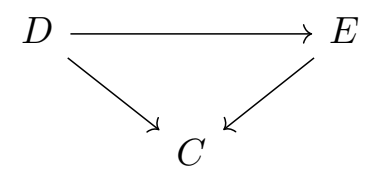

É comum mantermos a notação de um morfismo em $\mathcal{C} \downarrow C$ para um morfismo em $\mathcal{C}$. Mais precisamente, se $s: d \rightarrow e$ é um morfismo em $\mathcal{C} \downarrow C$, também chamamos de $s: D \rightarrow E$ o respectivo morfismo em $\mathcal{C}$.

No fim da seção 1.1 vimos um exemplo de categoria slice, veja que se $\mathcal{C}=$ Top é a categoria dos espaços topológicos, então dado um espaço topológico $X, \operatorname{Bund}(X)=$ Top $\downarrow$ $X$.

Teorema 2.4.7 Seja $\mathcal{E}$ um topos e $A$ objeto fixado de $\mathcal{E}$. Então a categoria slice $\mathcal{E} \downarrow A$ é um topos.

\section{Demonstração:}

O objeto terminal em $\mathcal{E} \downarrow A$ é simplesmente a identidade $i d_{A}: A \rightarrow A$ em $\mathcal{E}$.

Agora veremos que $\mathcal{E} \downarrow A$ possui todos os limites finitos. Sejam $f: F \rightarrow A$ e $g: G \rightarrow A$ morfismos em $\mathcal{E} \downarrow A$.

O produto de $f$ e $g$ em $\mathcal{E} \downarrow A$ é o pullback em $\mathcal{E}$, onde $p_{F}$ e $p_{G}$ são as projeções.

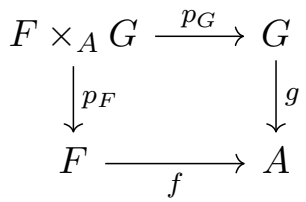

O equalizador de $f, g$ é o morfismo $e q(r, s) \rightarrow A$ onde $r, s: F \rightarrow G$ são morfismos em $\mathcal{E} \downarrow A$ e $e q(r, s)$ é o equalizador de $r$ e $s$.

Agora vamos mostrar que $\mathcal{E} \downarrow A$ possui classificador de subobjeto.

Seja $t_{A}: A \rightarrow \Omega$ a composta dada por $A \rightarrow 1 \stackrel{t}{\rightarrow} \Omega$ em $\mathcal{E}$.

Tome $f, g$ morfismos em $\mathcal{E} \downarrow A$ da forma $f: F \rightarrow A$ e $g: G \rightarrow A$. Verifica-se que $s: g \rightarrow f$ é um subobjeto de $\mathcal{E} \downarrow A$ quando $s: G \longmapsto F$ um mono em $\mathcal{E}$. O morfismo característico de $s$ em $\mathcal{E}$ é $\chi: F \rightarrow \Omega$.

Veremos que $(f, \chi)$ é morfismo característico de $s$ em $\mathcal{E} \downarrow A$.

Considerando $p_{\Omega}: A \times \Omega \rightarrow \Omega$ a projeção na segunda coordenada, obtemos o pullback abaixo:

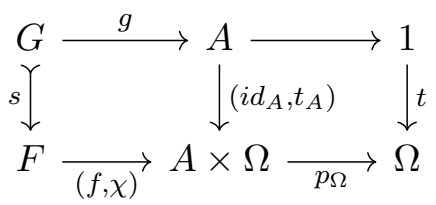

Temos também $\searrow_{f} \stackrel{(f, \chi)}{\longrightarrow} A \times \Omega$

do que isso, $(f, \chi)$ é morfismo característico de $s$ em $\mathcal{E} \downarrow A$ com classificador de subobjeto $p_{\Omega}: A \times \Omega \rightarrow \Omega$, pois o seguinte diagrama é um pullback em $\mathcal{E} \downarrow A$

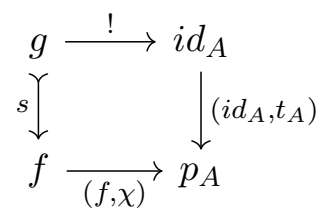

Verificar que $\mathcal{E} \downarrow A$ possui objetos exponenciais já é bem mais complicado que os casos acima e para fazer os devidos detalhes são necessários resultados que não enunciamos nessa Dissertação. Para ver essa demonstração completa indicamos [MLM94] (Capítulo IV, Seção 7, Teorema 1). 
Definição 2.4.8 Seja $f: B \rightarrow A$ um morfismo em um topos $\mathcal{E}$. Definimos $f^{*}: \mathcal{E} \downarrow A \rightarrow$ $\mathcal{E} \downarrow B$ como o funtor mudança de base se for obtido pelo pullback ao longo de $f$.

Veja que tomar o pullback ao longo de $f$ leva objetos de $\mathcal{E} \downarrow A$, digamos, $X \rightarrow A$ em objetos de $\mathcal{E} \downarrow B$, digamos, $X^{\prime} \rightarrow B$ :
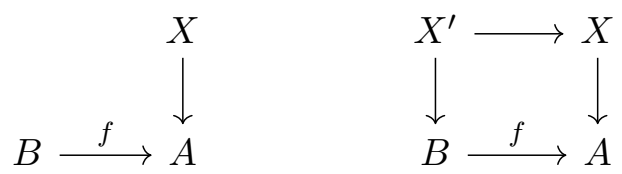

A definição da ação nos morfismos segue da propriedade universal dos pullbacks.

Teorema 2.4.9 Seja $f: B \rightarrow A$ um morfismo em um topos $\mathcal{E}$. Então $f^{*}: \mathcal{E} \downarrow A \rightarrow \mathcal{E} \downarrow B$ possui adjunto à esquerda $\sum_{f}$ e adjunto à direita $\prod_{f}$.

\section{Demonstração:}

O adjunto à esquerda é simplesmente a composição com $f$, isto é, dado $h: H \rightarrow B$ em $\mathcal{E} \downarrow B$ definimos $\sum_{f}(h)=f \circ h$ e verificar a adjunção é imediata. O interessante é verificar que possui adjunto à direita.

Vamos primeiro ver o que acontece quando $A=1$ é o objeto terminal. Nesse caso, $\mathcal{E} \downarrow A=\mathcal{E} \downarrow 1 \cong \mathcal{E}$ é um isomofismo e o funtor mudança de base de $f: B \rightarrow 1$ é dado por $-\times B: \mathcal{E} \rightarrow \mathcal{E} \downarrow B, G \mapsto\left(G \times B \stackrel{p_{B}}{\longrightarrow} B\right)$ onde $p_{B}$ é a projeção.

Seja $h: H \rightarrow B$ um morfismo em $\mathcal{E} \downarrow B$. Um $r: p_{B} \rightarrow h$ em $\mathcal{E} \downarrow B$ é um morfismo $r: G \times B \rightarrow H$ em $\mathcal{E}$ tal que $h \circ r=p_{B}$

Agora tomamos a transposta exponencial de $r, \widetilde{r}: G \rightarrow H^{B}$ onde o seguinte diagrama comuta

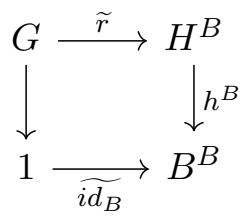

sendo que $\widetilde{i d_{B}}$ é a transposta exponencial da identidade $i d_{B}: 1 \times B=B \rightarrow B$.

Considerando o pullback $P$ de $h^{B}$ e $\overline{i d_{B}}$ obtemos o diagrama

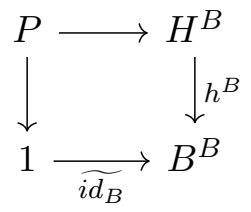

e um único morfismo $r^{\prime}: G \rightarrow P$ em correspondência com $\widetilde{r}$. Então esse pullback é o funtor adjunto à direita de $-\times B$.

Devemos voltar para o caso geral.

O morfismo $f: B \rightarrow A$ em $\mathcal{E}$ também pode ser visto como um objeto em $\mathcal{E} \downarrow A$, o qual denotaremos por $(f)$. Um objeto sobre $(f)$ é um diagrama comutativo determinado por um objeto em $\mathcal{E} \downarrow B$, digamos, $X \rightarrow B$ como o abaixo.

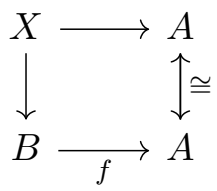

Analogamente ao processo de pegar um objeto $B$ em $\mathcal{E}$ e obter a categoria slice $\mathcal{E} \downarrow B$, podemos considerar um objeto $(f)$ de $\mathcal{E} \downarrow A$ e formar a categoria slice $(\mathcal{E} \downarrow A) \downarrow(f)$. Pela correspondência dada no último diagrama temos $(\mathcal{E} \downarrow A) \downarrow(f) \cong \mathcal{E} \downarrow B$. 
Assim, o funtor mudança de base é $-\times(f): \mathcal{E} \downarrow A \rightarrow(\mathcal{E} \downarrow A) \downarrow(f)$ e caímos no caso anterior onde podemos encontrar um pullback $\prod_{f}$ de $h^{(f)}$ e $\widetilde{i d_{(f)}}$ como adjunto à direita do funtor mudança de base.

Em breve usaremos um isomorfismo obtido no fim dessa demonstração para facilitar outros argumentos, por isso, merece destaque:

Proposição 2.4.10 Para todo morfismos $f: B \rightarrow A$ em $\mathcal{E}$, existe um isomorfismo de categorias $\mathcal{E} \downarrow B \cong(\mathcal{E} \downarrow A) \downarrow(f)$.

É hora de justificar o porquê de denotarmos o funtor mudança base da mesma forma como denotamos a imagem inversa. Como consequência do Teorema acima obtemos:

Corolário 2.4.11 Qualquer morfimo $f: B \rightarrow A$ em um topos $\mathcal{E}$ induz um morfismo geométrico $\mathcal{E} \downarrow B \rightarrow \mathcal{E} \downarrow A$ onde a imagem direta é $\prod_{f}$ e a imagem inversa é o funtor mudança de base.

Ou seja, o funtor mudança de base é, de fato, uma imagem inversa.

Teorema 2.4.12 Seja $f: B \rightarrow A$ um morfismo em um topos $\mathcal{E}$. Então $f^{*}: \mathcal{E} \downarrow A \rightarrow \mathcal{E} \downarrow B$ é um morfismo lógico.

\section{Demonstração:}

Um fato bem conhecido sobre adjuntos é que funtores que possuem adjuntos à esquerda preservam limites. Já vimos que o adjunto à esquerda existe, portanto, $f^{*}$ preserva limites (não apenas os finitos, preserva todos os limites pequenos).

Agora usamos a Proposição 2.4.10 e temos $f^{*}: \mathcal{E} \downarrow A \rightarrow(\mathcal{E} \downarrow A) \downarrow(f)$. Pelo Teorema 2.4.7, $\mathcal{E} \downarrow A$ é um topos, então chamamos $\mathcal{F}=\mathcal{E} \downarrow A$ e assim basta mostrar que, dado $X$ objeto de $\mathcal{F}$, o funtor $X^{*}: \mathcal{F} \rightarrow \mathcal{F} \downarrow X, Y \mapsto\left(p_{X}: X \times Y \rightarrow X\right)$ preserva classificador de subobjeto e exponencial (pois, em particular, $(f)$ é um objeto de $\mathcal{E} \downarrow A$ ).

Vimos no Teorema 2.4.7 que um classificador de subobjeto em $\mathcal{F} \downarrow X$ é da forma $p_{X}: X \times \Omega \rightarrow X$, então é imediato que preserva classificador de subobjeto. Vamos provar que $X^{*}$ preserva exponencial:

Sejam $p_{X}^{D}: X \times D \rightarrow X$ e $p_{X}^{C}: X \times C \rightarrow X$, devemos verificar que $p_{X}^{D^{C}}: X \times D^{C} \rightarrow X$ é exponencial de $p_{X}^{D}$ por $p_{X}^{C}$ em $\mathcal{F} \downarrow X$.

Seja $\alpha: E \rightarrow X$ um morfismo em $\mathcal{F}$ e faça o pullback com $p_{X}^{C}$, resultando no seguinte diagrama

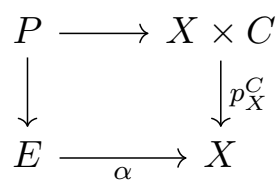

Fazendo o produto $\alpha \times p_{X}^{C}$ obtemos o diagrama comutativo:

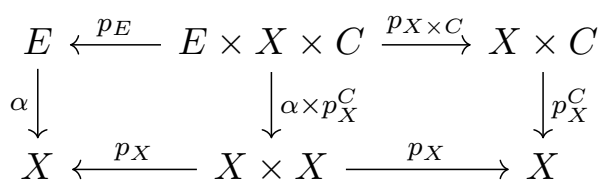

Então $\alpha p_{X}^{C}$ está em $\mathcal{F} \downarrow X$ e temos que para todo $\alpha \times p_{X}^{C} \rightarrow p_{X}^{D}$ existe um único $\alpha \rightarrow\left(p_{X}^{D}\right)^{\left(p_{X}^{C}\right)}$ que torna o diagrama abaixo comutativo

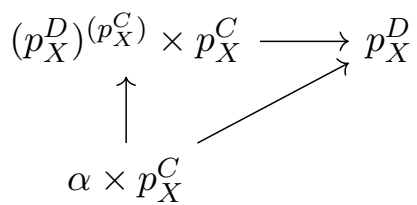




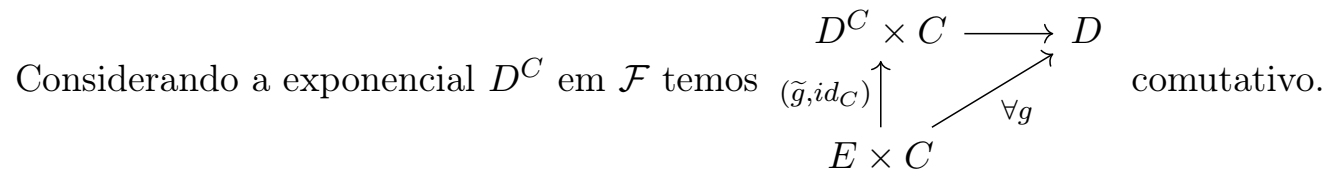

Note que cada $g$ induz morfismo $\alpha \times p_{X}^{C} \rightarrow p_{X}^{D}$ e assim existe um único morfismo $(\alpha, \widetilde{g}): \alpha \rightarrow p_{X}^{C}$ que faz o seguinte diagrama comutar:

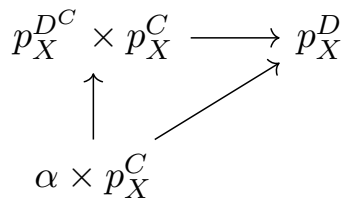

Então $p_{X}^{D^{C}} \cong\left(p_{X}^{D}\right)^{\left(p_{X}^{C}\right)}$.

Corolário 2.4.13 Em um topos colimites são universais, isto é, colimites são preservados pelo funtor pullback.

\section{Demonstração:}

Analogamente ao que ocore para à esquerda, funtores que possuem adjuntos à direita preservam colimites. Já vimos que o adjunto à direita $\prod_{f}$ existe, portanto, $f^{*}$ preserva colimites.

Esse corolário nos permite apresentar uma demonstração de que todo morfismo em um topos se fatora em epi e mono por uma construção análoga ao que fizemos para categorias abelianas.

Teorema 2.4.14 Qualquer morfismo em um topos pode ser fatorado por um epi q seguido de um mono $i$ e essa fatoração é única a menos de isomorfismo.

\section{Demonstração:}

Começamos verificando a existência da fatoração. Considere o morfismo $f: X \rightarrow Y$ e tome o kernelpar $(a, b)$ de $f$

$$
R \underset{b}{\stackrel{a}{\longrightarrow}} X \stackrel{f}{\longrightarrow} Y
$$

Considere também $q$ o coequalizador de $(a, b)^{3}$. Então temos

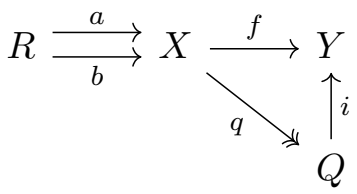

Assim já temos que $q$ é epi, vamos ver que $i$ é mono.

Seja $c$ e $d$ um par de morfismos tal que $T \underset{d}{\stackrel{c}{\longrightarrow}} Q$ com $i c=i d$.

Tomando o pullback abaixo, obtemos $f g=i q g=i c e=i d e=f h$.

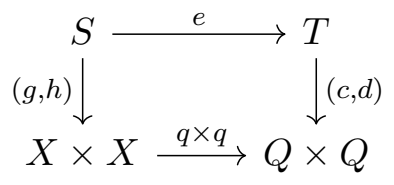

Então $(g, h)$ se fatora ao longo de $(a, b): R \longmapsto X \times X$, digamos, por um $S \stackrel{k}{\rightarrow} R$.

Ainda, $c e=q g=q a k=q b k=q h=d e$.

\footnotetext{
${ }^{3}$ Observe como a ideia dessa demonstração é similar ao caso das categorias abelianas. Antes usamos o cokernel do kernel de $f$, agora consideramos o coequalizador do kernelpar de $f$
} 
Veja ainda que $q \times q=\left(q \times i d_{Q}\right) \circ\left(i d_{X} \times q\right)$. Como $q$ é epi, tanto $\left(q \times i d_{Q}\right)$ quanto $\left(i d_{X} \times q\right)$ são epis, logo, $q \times q$ é epi. Como o diagrama acima é um pullback, pelo Corolário 2.4.13, e também é epi.

Vimos que $c e=d e$, como é é epi, $c=d$, portanto, $i$ é mono.

Resta verificar a unicidade da fatoração.

Considere uma outra fatoração em epi e mono $f=i^{\prime} \circ q^{\prime}$.

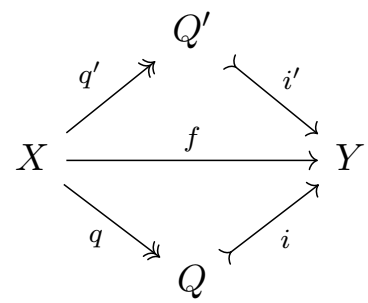

Temos $i^{\prime} \circ q^{\prime} \circ a=i^{\prime} \circ q^{\prime} \circ b$. Como $i^{\prime}$ é mono, $q^{\prime} \circ a=q^{\prime} \circ b$. Lembrando que $(q, Q)$ é o coequalizador de $(a, b)$, pela propriedade universal, existe um único morfismo $u: Q \rightarrow Q^{\prime}$ tal que $u \circ q=q^{\prime}$. Assim, $u$ é epi pois $q^{\prime}$ é epi.

Por outro lado, $i \circ q=p=i^{\prime} \circ q^{\prime}=i^{\prime} \circ u \circ q$. Como $q$ é epi, temos $i=i^{\prime} \circ u$. Entãi $u$ é mono, pois $i$ é mono. Pelo Corolário 2.3.11, $u$ é isomorfismo.

Isso significa que um morfismo $f$ em um topos sempre se fatora pela sua imagem.

Corolário 2.4.15 Em um topos todo epimorfismo é um coequalizador (isso é o mesmo que dizer que todo epimorfirsmo é regular).

\section{Demonstração:}

Veremos que um epi é o coequalizador do seu kernel-par. Vamos aproveitar o diagrama do Teorema acima:

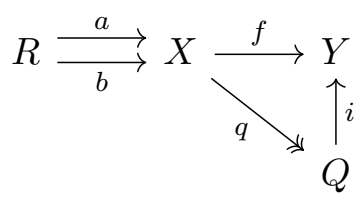

O morfismo $q$ é o coequalizador do par $(a, b)$ que é kernelpar de $f$. Suponha $f$ epi, então $i \circ q$ é epi, logo, $i$ é epi. Na demonstração vimos que $i$ é mono, pelo Corolário 2.3.11, $i$ é iso. Assim concluímos que $f$ é, assim como $q$, coequalizador.

Ainda, uma vez que garantimos a fatoração pela imagem para todo morfismo, obetemos um lema que nos será útil na próxima seção:

Lema 2.4.16 Em um topos, a união (também visto como o supremo) de dois subobjetos sempre existe.

\section{Demonstração:}

Sejam $r: R \longmapsto A$ e $s: S \longmapsto A$ dois subojetos de $A$. Considere $R \coprod S$ o coproduto e seja $f: R \amalg S \rightarrow A$. Levando em conta a fatoração de $f$ pela imagem e os morfismos $s_{R}$ e $s_{S}$ do coproduto tais que $f \circ s_{R}=r$ e $f \circ s_{S}=s$ temos um diagrama da seguinte forma

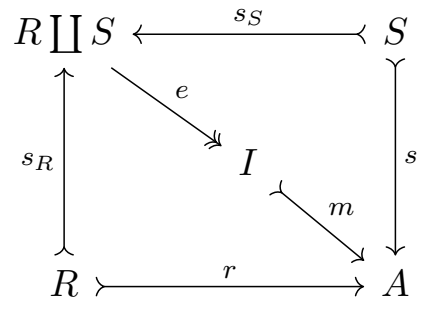


Como $s$ e $r$ são monos, temos $e \circ s_{S}: S \longmapsto I$ e $e \circ s_{R}: R \longmapsto$ monomorfismos, então $I$ é subobjeto de $A$ que contem $R$ e $S$. Além disso, $I$ é o menor subojeto de $A$ que contem $R$ e $S$, pois tomamos a fatoração pela imagem, $\operatorname{logo}, I=R \cup S$.

Faltam mais alguns resultados para podermos apresentar uma caracterização de topos de Grothendieck.

Proposição 2.4.17 Em um topos $\mathcal{E}$ o objeto inicial 0 é estrito, isto é, qualquer morfismo $X \rightarrow 0$ é um isomorfismo.

\section{Demonstração:}

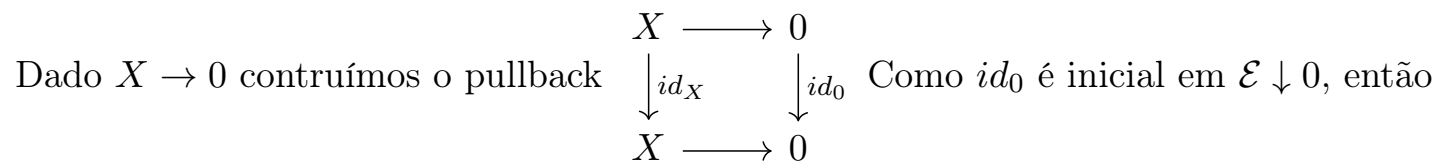
$i d_{X}$ é inicial em $\mathcal{E} \downarrow X$ (os colimites são universais). Por fim, como $i d_{X}$ é objeto termina em $\mathcal{E} \downarrow X$, temos que $\mathcal{E} \downarrow X$ é o topos trivial, isto é, possui um único objeto e um único morfismo.

Corolário 2.4.18 Seja $\mathcal{E}$ um topos. Todo morfismo $0 \rightarrow X$ em $\mathcal{E}$ é um monomorfismo.

\section{Demonstração:}

Tome $g_{1}$ e $g_{2}$ morfismos da forma $Y \rightarrow 0$ tais que $f \circ g_{1}=f \circ g_{2}$. Pelo Corolário 2.4.6, $g_{1}$ e $g_{2}$ são isomorfismos, logo, possuem inversas $g_{1}^{-1}$ e $g_{2}^{-1}$, respectivamente, que são da forma $0 \rightarrow Y$. Contudo, um morfismo dessa forma é único e, portanto $g_{1}^{-1}=g_{2}^{-1}$. Então $g_{1}=g_{2}$ e concluímos que $0 \rightarrow X$ é mono pois, é claro, $f \circ g_{1}=f \circ g_{2}$ implica $g_{1}=g_{2}$.

Definição 2.4.19 Seja $X$ coproduto de uma família de objetos de $\left\{X_{i} \mid i \in I\right\}$ de $\mathcal{E}, I$ conjunto de índices. Dizemos que $X$ é disjunto se:

1. Cada morfismo canônico do coproduto $\nu_{i}: X_{i} \rightarrow X$ é mono.

2. Para cada par de índices distintos $(i, j)$ o pullback $X_{i} \times_{X} X_{j}$ é um objeto inicial em $\mathcal{E}$.

Proposição 2.4.20 Em um topos, coprodutos são disjuntos.

\section{Demonstração:}

Para ilustrar faremos o caso do copruduto de dois objetos $X$ e $Y$. Por definição de coproduto, o seguinte diagrama é um pushout.

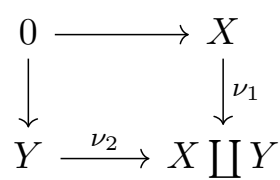

Pela Proposição 2.4.13, os morfismos $0 \rightarrow X$ e $0 \rightarrow Y$ são monos. Assim, pela Proposição 2.3.18, temos que pushout é também um pullback e $\nu_{1}, \nu_{2}$ são monos, portanto, as duas condições de disjunto estão satisfeitas.

Vamos definir o gerador de uma categoria.

Definição 2.4.21 Seja $G=\left\{G_{i} \mid i \in I\right\}$ uma família de objetos em uma categoria $\mathcal{C}$. Dizemos que $G$ é gerador de $\mathcal{C}$ se, dados dois morfismos $f, g: X \rightarrow Y$ em $\mathcal{C}, f \neq g$, existe algum índice $i \in I$ e algum morfismo $h: G_{i} \rightarrow X$ tal que $f \circ g \neq g \circ h$.

Já vimos que todo topos de Grothendieck é topos elementar, agora apresentamos um resultado de caracterização dos topos de Grothendieck.

Teorema 2.4.22 (Teorema de Giraud): Uma categoria $\mathcal{E}$ é um topos de Grothendieck se, e somente se, as seguintes condições são satisfeitas 
(i) $\mathcal{E}$ é localmente pequena com um conjunto de geradores;

(ii) $\mathcal{E}$ possui limites finitos;

(iii) $\mathcal{E}$ possui coprodutos pequenos, os quais são disjuntos e universais;

(iv) Toda relação de equivalência em $\mathcal{E}$ possui coequalizador e esse coequalizador é universal

(v) Toda relação de equivalência em $\mathcal{E}$ é efetiva

(vi) Todo epimorfismo em $\mathcal{E}$ é regular.

\section{Demonstração:}

Essa é uma demonstração extremamente técnica que acreditamos não valer a pena transcrevê-la, mas podemos descrever a ideia da prova. Se $\mathcal{E}$ for topos de Grothendieck então fazemos $\mathcal{E}=S h(\mathcal{C}, J)$ e pode-se mostrar que as seis condições são satisfeitas.

Para a volta assumimos que as condições valem em $\mathcal{E}$ e precisamos definir uma categoria $\mathcal{C}$ e uma topologia de Grothendieck $J$ tal que $S h(\mathcal{C}, J)$ seja equivalente a $\mathcal{E}$.

O que se faz é definir $\mathcal{C}$ como uma subcategoria plena de $\mathcal{E}$ gerada por um conjunto arbitrário de geradores. Para cada objeto $C$ de $\mathcal{C}$ definimos $J(C)$ como o conjuntos dos subfuntores $R \longmapsto \operatorname{Hom}(-, C)$ tais que $\coprod_{X} R(X)$, com $X$ objeto de $\mathcal{C}$ é uma família de epimorfismo em $\mathcal{C}$.

Para ver detalhes indicamos duas referências: o Teorema 3.6.1 de [Bor94c] ou Teorema 1 do Apêndice de [MLM94].

Agora introduziremos a noção que generaliza categorias pequenas, as categorias internas a um topos. Categorias pequenas consistem de um conjunto de objetos e um conjunto de morfismos, mas categorias internas consistem de um objeto de objetos e um objeto de morfismos com morfismos e axiomas para esses morfismos.

Definição 2.4.23 Seja $\mathcal{E}$ categoria com pullbacks. Uma categoria interna $\mathcal{C}$ em $\mathcal{E}$ consiste de objetos $\mathcal{C}_{0}$ e $\mathcal{C}_{1}$, de $\mathcal{E}$, respectivamente, objeto de objetos e objeto de morfismos; quatro morfismos $d_{0}: \mathcal{C}_{1} \rightarrow \mathcal{C}_{0}, d_{1}: \mathcal{C}_{1} \rightarrow \mathcal{C}_{0}, e: \mathcal{C}_{0} \rightarrow \mathcal{C}_{1}$ e $m: \mathcal{C}_{2} \rightarrow \mathcal{C}_{1}$, conhecidos como domínio, codomínio, identidade e composição, respectivamente, onde $\mathcal{C}_{2}=\mathcal{C}_{1} \times \mathcal{C}_{0} \mathcal{C}_{1} \rightarrow \mathcal{C}_{1}$ $\mathcal{C}_{2} \stackrel{\pi_{2}}{\longrightarrow} \mathcal{C}_{1}$
$\mathcal{C}_{1} \stackrel{d_{0}}{\longrightarrow} \mathcal{C}_{0}$

1. $d_{0} e=d_{1} e=i d$

2. $d_{0} m=d_{0} \pi_{2}$ e $d_{1} m=d_{1} \pi_{1}$

3. O diagrama $\underset{m \times i d}{\stackrel{\mathcal{C}_{3}}{\mathcal{C}_{2} \stackrel{i d \times m}{\longrightarrow}} \mathcal{C}_{2}} \underset{\mathcal{C}_{2}}{\longrightarrow} \mathcal{C}_{1}$ comuta, onde $\mathcal{C}_{3}=\mathcal{C}_{1} \times \mathcal{C}_{0} \mathcal{C}_{1} \times \mathcal{C}_{0} \mathcal{C}_{1}$

$$
\mathcal{C}_{1} \times \mathcal{C}_{0} \mathcal{C}_{0} \stackrel{i d \times e}{\longrightarrow} \mathcal{C}_{2} \stackrel{e \times i d}{\stackrel{\text { ex }}{\longleftarrow}} \mathcal{C}_{0} \times \mathcal{C}_{0} \mathcal{C}_{1}
$$

4. O diagrama

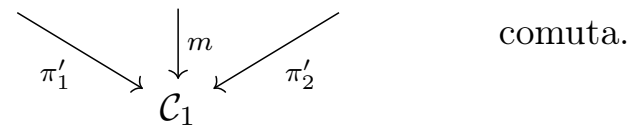

Note que a definição acima é uma forma de definir uma categoria em termos de diagramas. Esse mesmo procedimento se repetirá ao longo do trabalho.

É natural querermos para categorias internas o que temos em categorias, o próximo passo não poderia ser outro: dizer o que é um funtor entre categorias internas. 
Definição 2.4.24 Sejam $\mathcal{C}=\left(\mathcal{C}_{0}, \mathcal{C}_{1}, d_{0}, d_{1}, e, m\right)$ e $\mathcal{D}=\left(\mathcal{D}_{0}, \mathcal{D}_{1}, d_{0}^{\prime}, d_{1}^{\prime}, e^{\prime}, m^{\prime}\right)$ categorias internas. Dizemos que o funtor $\left(G_{0}, G_{1}\right)=G: \mathcal{C} \rightarrow \mathcal{D}$ é interno se $G_{0}: \mathcal{C}_{0} \rightarrow \mathcal{D}_{0}$ e $G_{1}: \mathcal{C}_{1} \rightarrow \mathcal{D}_{1}$ são morfismos em $\mathcal{E}$ que comutam com $e, d_{0}, d_{1}$ e $m$.

Agora vamos introduzir a noção de diagrama interno, também chamada de objeto esquerdo, provavelmente pela sua semelhança com às ações à esquerda de um grupo.

A partir desta parte vamos assumir que $\mathcal{E}$ é um topos.

Definição 2.4.25 Seja $\mathcal{C}$ uma categoria interna em $\mathcal{E}$. Um diagrama interno $F$ em $\mathcal{C}$ (ou $\mathcal{C}$-objeto esquerdo em $\mathcal{E}$ ) é composto por um objeto $\pi: F \rightarrow \mathcal{C}_{0}$ em $\mathcal{E} \downarrow \mathcal{C}_{0}$ equipado com uma ação $\mu: \mathcal{C}_{1} \times{ }_{\mathcal{C}_{0}} F \rightarrow F$ de $\mathcal{C}$ em $\mathcal{E}$ de forma que $\mathcal{C}_{1}$ é visto como objeto sobre $\mathcal{C}_{0}$ (i.e., está em $\mathcal{E} \downarrow \mathcal{C}_{0}$ ), usando $d_{0}$ no pullback $\mathcal{C}_{1} \times \mathcal{C}_{0} F$. Mais ainda, os três diagramas a seguir devem comutar:
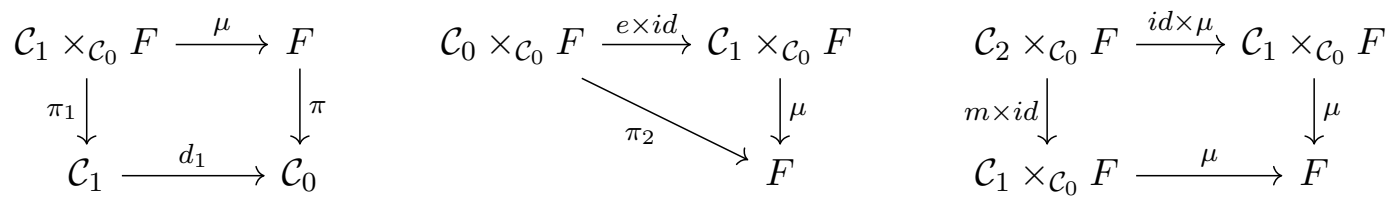

Note que toda categoria interna determina diagrama interno onde $\left(\pi: F \rightarrow \mathcal{C}_{0}\right)$ é $\left(d_{1}: \mathcal{C}_{1} \rightarrow \mathcal{C}_{0}\right)$ e $\mu=m$.

Queremos construir a categoria de todos os diagramas internos, então precisamos dizer como são os morfismos entre esses diagramas.

Definição 2.4.26 Sejam $(F, \pi, \mu)$ e $\left(F^{\prime}, \pi^{\prime}, \mu^{\prime}\right)$ diagramas internos em $\mathcal{C}$ categoria interna de $\mathcal{E}$. Um morfismo de diagramas internos é um morfismo $\phi: F \rightarrow F^{\prime}$ em $\mathcal{E}$ que preserva a estrutura, isto é, os diagramas abaixo comutam
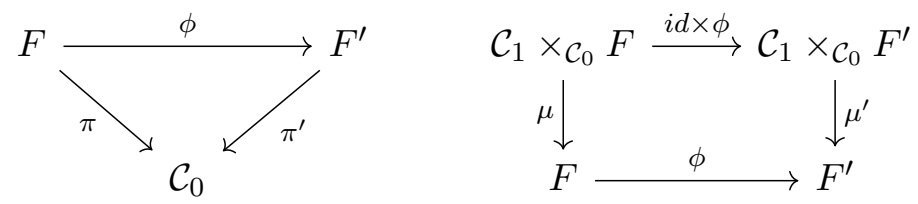

Com isso chamamos de $D_{\mathcal{E}}^{\mathcal{C}}$ a categoria dos diagramas internos $\mathcal{C}$ em $\mathcal{E}$

Sem nos aprofundarmos nas definições e resultados intermediários envolvidos, é possível verificar que existe forte relação entre a categoria interna ao topos e a categoria dos diagramas internos da seguinte forma:

Proposição 2.4.27 Seja $\mathcal{E}$ um topos e $\mathcal{C}$ uma categoria interna em $\mathcal{E}$. Então o funtor esquecimento $U: D_{\mathcal{E}}^{\mathcal{C}} \rightarrow \mathcal{E} \downarrow C_{0}$ é comonádico.

\section{Demonstração:}

Proposição 2.31 de [Joh14]

Com isso devemos ter esboçado o suficiente da utilidade da categoria Slice e introduzido ideias que serão usadas novamente mais tarde (redefinir conceitos conhecidos apenas em termos de diagramas).

\section{5 Álgebras de Heyting em um Topos}

As Álgebras de Heyting são para um Topos o que as Álgebras de Boole são para Sets. Essa seção começa a introduzir um pouco do lado Lógico do topos, aqui usamos [MLM94] e [Bor94c].

Definição 2.5.1 Seja $\mathcal{C}$ uma categoria com limites finitos. Um reticulado (limitado) em $\mathcal{C}$ é um objeto $L$ de $\mathcal{C}$ munido de dois morfismos em $\mathcal{C}$, inf e sup, respectivamente: 


$$
\bigwedge: L \times L \longrightarrow L \quad \bigvee: L \times L \longrightarrow L
$$

satisfazendo as leis de um reticulado no sentido usual, isto é, os seguintes diagramas comutam

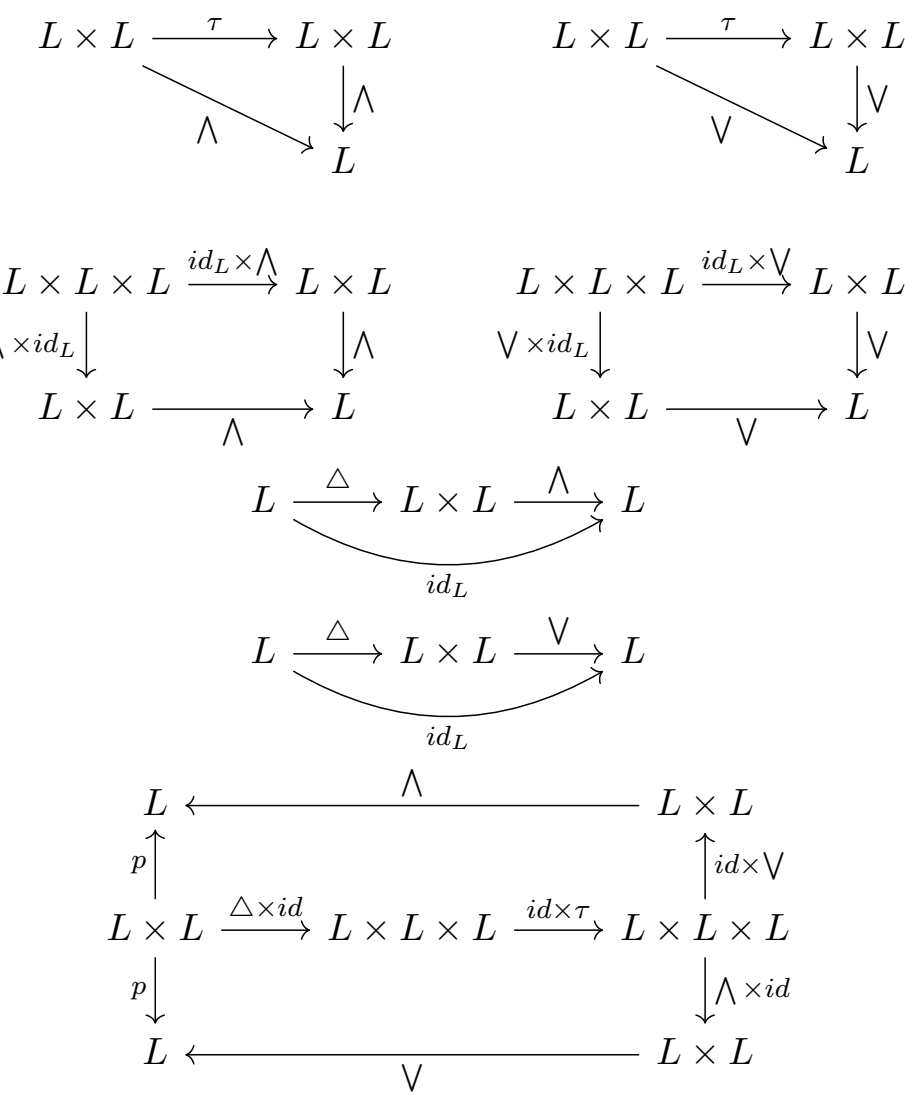

onde $p$ é a projeção, $\triangle$ é o morfismo diagonal, $\tau$ é o mapa "twist" que troca os fatores do produto e assumimos $L \times(L \times L) \cong(L \times L) \times L$ é um isomorfismo.

Caso não esteja claro, os dois primeiros diagramas representam a comutatividade de inf e sup, os dois seguintes a associativa, o quinto e o sexto, a idempotência e o último diagrama captura a lei da absorção $x \wedge(y \vee x)=x=(x \wedge y) \vee$, onde o lado esquerdo da igualdade é representado pela parte de cima do diagrama e o lado direito da igualdade é dado pela comutatividade da parte de baixo do diagrama.

Além disso, falta algo para traduzir os elementos zero e o um. Temos que $L$ possui zero e um quando existem morfismos $\top: 1 \rightarrow L$ e $\perp: 1 \rightarrow L$, onde 1 é objeto terminal de $\mathcal{C}$, satisfazendo:

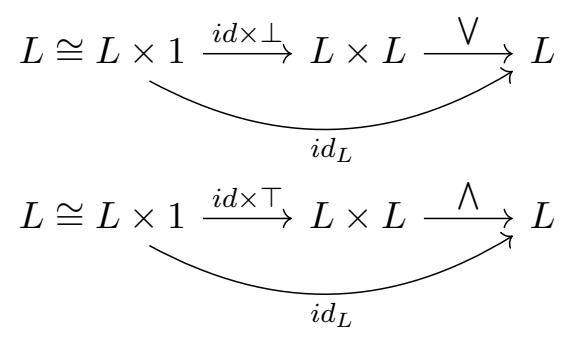

Note que poderíamos trocar as identidades pelas projeções $p_{L}: L \times 1 \cong L$

Em outras palavras, valem $x \wedge \perp=x$ e $x \vee \top=x$.

Sabendo como são os reticulados limitados, podemos definir a Álgebra de Heyting em uma categoria.

Definição 2.5.2 Sejam $\mathcal{C}$ uma categoria com limites finitos e $L$ um reticulado limitado, dizemos que $L$ é uma Álgebra de Heyting interna a $\mathcal{C}$ se temos um morfismo $\Longrightarrow$ : $L \times L \rightarrow L$ satisfazendo as seguintes leis porém em termos dos diagramas: 
1. $(x \Longrightarrow x)=1$

2. $x \wedge(x \Longrightarrow y)=x \wedge y$ e $y \wedge(x \Longrightarrow y)=y$

3. $x \Longrightarrow(y \wedge z)=(x \Longrightarrow y) \wedge(x \Longrightarrow z)$

Isto é, os seguintes diagramas comutam:

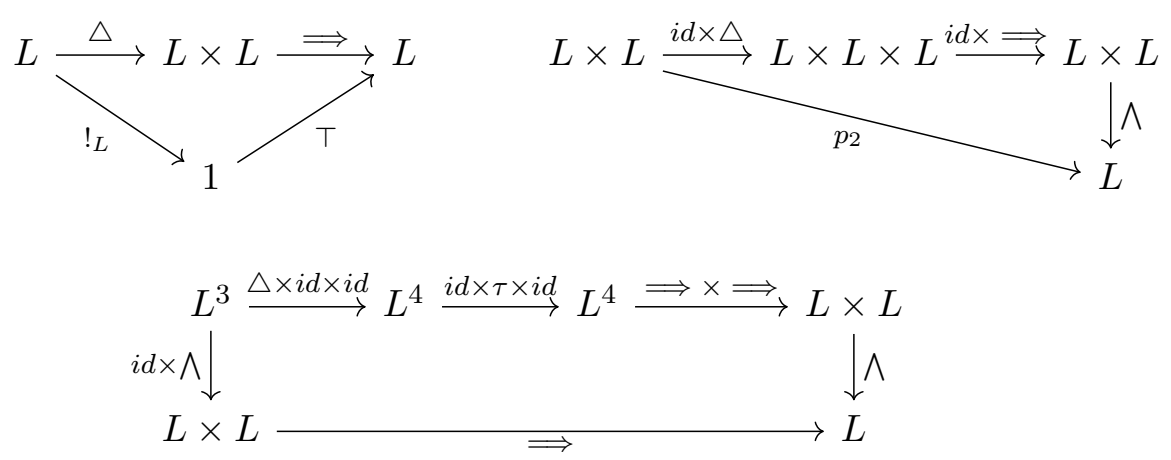

onde $L^{3}=L \times L \times L$ e $L^{4}=L \times L \times L \times L$.

A negação $\neg: L \rightarrow L$ é definida pelo seguinte diagrama comutativo:

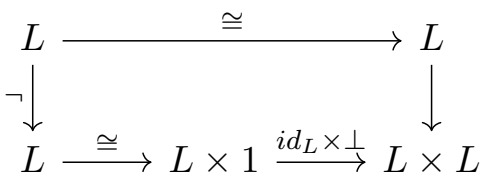

Agora vamos definir um homomorfismo de álgebras de Heyting interna.

Definição 2.5.3 Sejam $L, L^{\prime}$ álgebras de Heyting internas e $f: L \rightarrow L^{\prime}$ morfismo em $\mathcal{E}$. Dizemos que $f$ é um homomorfismo de álgebras de Heyting (ou mesmo de reticulados limitados caso $L$ e $L^{\prime}$ sejam apenas reticulados internos) se esse morfismo comuta com todas operações envolvidas, isto é, os diagramas a seguir comutam.

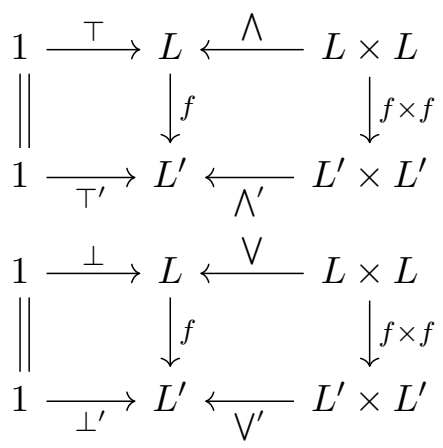

Note que acima faltou apenas o diagrama da implicação. Para fazê-lo basta pegar o quadrado do lado direito de qualquer um dos retânculos acima e trocar $\wedge$ (ou $\bigvee$, dependendo de qual escolheu) por $\Longrightarrow$.

Veja ainda que é possível encontrar uma relação de ordem nos reticulados internos, $x \leq y \Longleftrightarrow x \wedge y=x$. Para isso definimos $\leq_{L}$, um subobjeto de $L \times L$, como o equalizador abaixo:

$$
\leq_{L} \stackrel{e}{\longrightarrow} L \times L \underset{\pi_{1}}{\stackrel{\wedge}{\longrightarrow}} L
$$


Desta forma temos que $\left(L, \leq_{L}\right)$ é uma ordem parcial interna. Para verificar isso teríamos que provar as propriedades reflexiva, transitiva e anti-simétrica em sua forma interna, isto é, nos moldes da descrição diagramática de relações de equivalência (Definição 2.3.10). Isso está feito no Capítulo IV, seção 8 de [MLM94].

Queremos ver a relação entre topos e Álgebras de Heyting. Faremos isso em três passos. Primeiro mostraremos que $S u b(A)$ é um reticulado, depois, que é uma Álgebra de Heyting. Estamos falando aqui das versões usuais, não internas, ou como vamos passar a chamar: a versão externa. Por fim, veremos que as exponenciais $\Omega^{A}$ são quem carregam a estrutura de Álgebra de Heyting interna.

Observação 2.5.4 Toda Álgebra de Heyting é uma categoria cartesianamente fechada onde o produto coincide com interseção e a exponencial coincide com a implicação.

Lema 2.5.5 Seja $A$ um objeto de um topos $\mathcal{E}$. Temos que o conjunto parcialmente ordenado $\operatorname{Sub}(A)$ é um reticulado e, dado um morfismo $f: A \rightarrow B$, seu pullback, $f^{-1}$ : $S u b(B) \rightarrow S u b(A)$, é um morfismo de conjuntos parcialmente ordenados, isto é, um funtor. Além disso, $f^{-1}$ possui adjunto à esquerda $\exists_{f}$.

\section{Demonstração:}

Dados dois subobjetos $S \longmapsto A$ e $T \longmapsto A$, garantimos a intersecção pelo pullback

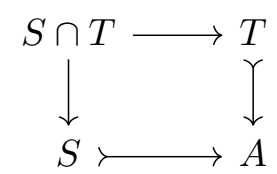

Pelo Lema 2.4.16 também conseguimos garantir a existência da união $S \cup T$. Dessa forma temos:

$$
S \wedge T=\inf \{S, T\}=S \cap T \text { e } S \bigvee T=\sup \{S, T\}=S \cup T
$$

Assim, as propriedades equacionais do reticulado são satisfeitas, restando apenas dizer quem são o 0 e o $1 \mathrm{em} S u b(A)$. O zero é simplesmente $0 \longmapsto A$ e o um é $A \rightarrow A$, onde o primeiro morfismo é mono pelo Corolário 2.4.18. Com isso, é claro que $0 \bigvee S=S$ e $1 \bigwedge S=S$. Então concluímos que $S u b(A)$ é um reticulado limitado.

Agora considere $f: A \rightarrow B, B$ objeto de $\mathcal{E}$.

Sabemos que pullbacks preservam inclusões de subobjetos e o pullback de um mono $T^{\prime} \longmapsto B$ ao longo de $f$ é necessariamente um mono $S \longmapsto A$, logo, $f^{-1}$ é um morfismo de conjuntos parcialmente ordenados.

Resta construir o adjunto à esquerda de $f^{-1}$. Seja $u: S \longmapsto A$ um subobjeto. A imagem de $u$ sobre $f$ é a imagem da composição $f \circ u$, portanto, é um subobjeto de $B$. Escrevemos a imagem de $f \circ u$ como $m: \exists_{f} S \longmapsto B$, onde $f \circ u=m \circ e$ para algum epi $e$, isto é, o seguinte diagrama comuta

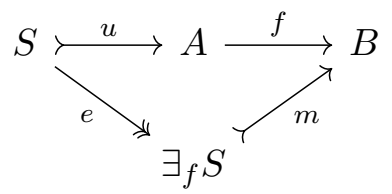

Seja $v: T \longmapsto B$ um subobjeto. O seu pullback ao longo de $f, f^{-1} T$, nos dá o diagrama:

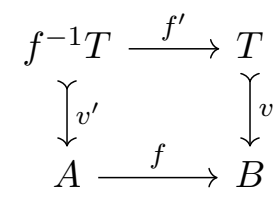


O que faremos é "colar" um diagrama à esquerda do acima para fazer $S$ aparecer e outro à direita, fazendo $\exists_{f} S$ aparecer. Através dessa união de três diagramas mostraremos que $S u b(B)\left(\exists_{f} S, T\right) \rightarrow S u b(A)\left(S, f^{-1} T\right)$ é uma adjunção, a qual chamaremos de $\exists_{f}$. Então tenha em mente o seguinte diagrama em $\mathcal{E}$.

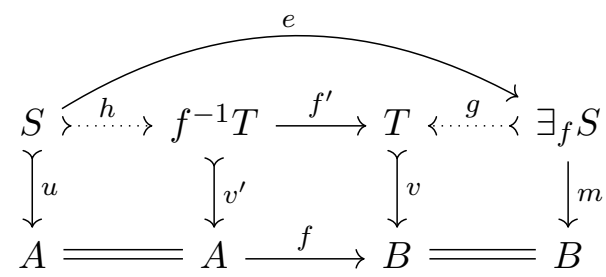

Note que a parte mais externa desse diagrama comuta pois $f \circ u=m \circ e$. Tome um morfismo $g$ em $\operatorname{Sub}(B)\left(\exists_{f} S, T\right)$, temos que $v \circ g=m$, pela definição de ordem $\leq$ em $\operatorname{Sub}(B)$. Note ainda que $g$ é único pois $v$ é mono e $g$ é monos pois $m$ é mono. Compondo com $e$ dos dois lados obtemos $v \circ g \circ e=m \circ e=f \circ u$, ou seja, algo da forma

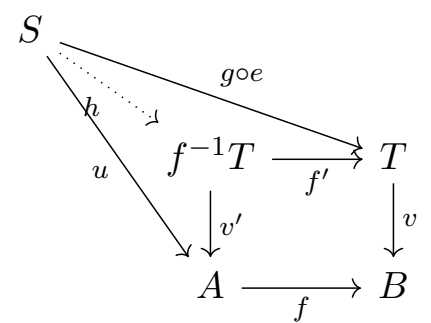

Como o diagrama central é pullback, existe uma única $h: S \rightarrow f^{-1} T$ tal que $u=v^{\prime} \circ h$ e $g \circ e=f^{\prime} \circ h$. Mas o importante é que $h \in \operatorname{Hom}_{A}\left(S, f^{-1} T\right)$.

Agora tome $h \in \operatorname{Hom}_{A}\left(S, f^{-1} T\right)$ subobjeto. Note que podemos fatorar $f \circ u=m \circ e$ por uma composição $v \circ g \circ e$ para algum $g: \exists_{f} S \rightarrow T$. Então a $h$ que tomamos é única, novamente pela propriedade universal do pullback. Como $m$ é imagem de $f \circ u, m=v \circ g$. Suponha que exista outro morfismo $g^{\prime}$ com $m=v g^{\prime}$. Nesse caso, $v g=v g^{\prime}$, como $v$ é mono, $g=g^{\prime}$. Portanto, $g$ é única e pertence a $\operatorname{Hom}_{B}\left(\exists_{f} S, T\right)$.

Provamos que $\operatorname{Hom}_{B}\left(\exists_{f} S, T\right) \rightarrow \operatorname{Hom}_{A}\left(S, f^{-1} T\right)$, com $g \mapsto h$ é uma bijeção, como queríamos.

Teorema 2.5.6 Seja A um objeto de um topos $\mathcal{E}$. Temos que $S u b(A)$ possui uma estrutura de Álgebra de Heyting. Ainda mais, essa estrutura é natural em $A$, no sentido que o pullback ao longo de qualquer morfismo $f: A \rightarrow B$ induz $f^{-1}: \operatorname{Sub}(B) \rightarrow S u b(A)$, um homomorfismo de álgebras de Heyting.

\section{Demonstração:}

Sejam $r: R \longmapsto A, s: S \longmapsto A, t: T \longmapsto A$ subobjetos de $A$ e considere seus respectivos morfismos característicos $\chi_{R}, \chi_{S}, \chi_{T}: A \rightarrow \Omega$.

A intersecção, obtida pelo pullback, $S \cap T \longmapsto A$ nos fornece outro subobjeto de $A$ cujo morfismo característico é $\chi_{S \cap T}: A \rightarrow \Omega$. Com isso definimos o subobjeto $(S \Longrightarrow T) \rightarrow A$ como o equalizador de $\chi_{S \cap T}$ e $\chi_{S}$.

$$
(S \Longrightarrow T) \succ A \stackrel{\chi_{S \cap T}}{\underset{\chi_{S}}{\longrightarrow}} \Omega
$$

Faça o pullback de $S \cap T$ ao longo de $r$, obtemos o subobjeto $R \cap(S \cap T)$ e ele é classificado por $\chi_{S \cap T} \circ r$, como podemos ver pelo diagrama abaixo (usando o Lema do Pullback)

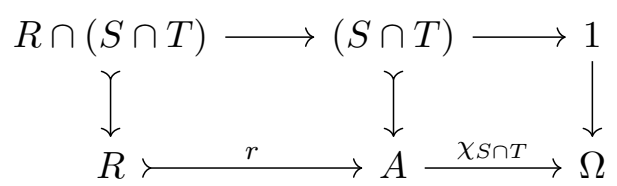


Analogamente, fazemos o pullback de $S$ ao longo de $r$ e obtemos o subobjeto $R \cap S$ classificado por $\chi_{S} \circ r$. Por definição de equalizador $R \leq(S \Longrightarrow T)$ se, e somente se, $\chi_{S} \circ r=\chi_{S \cap T} \circ r$. Pelos diagramas do classificadores essa igualdade ocorre se e só se $R \cap S=R \cap(S \cap T)$ e, é claro,

$$
R \cap S=R \cap(S \cap T) \Longleftrightarrow R \cap S \leq R \cap S \cap T \Longleftrightarrow R \cap S \leq T
$$

Concluímos que $R \leq(S \Longrightarrow T)$ se, e só se, $R \cap S \leq T$, logo, $S u b(A)$ é álgebra de Heyting.

Agora tome $f: A \rightarrow B$ um morfismo em $\mathcal{E}$. Seja $i_{A}: S u b(A) \rightarrow \mathcal{E} \downarrow A$ a inclusão que identifica subobjetos de $A$ em $\mathcal{E}$ com subobjetos $i d_{A}$ em $\mathcal{E} \downarrow A$. Assim, sabendo que $\operatorname{Sub}(B)=\operatorname{Mono}(B) / \cong$, construímos o seguinte diagrama comutativo

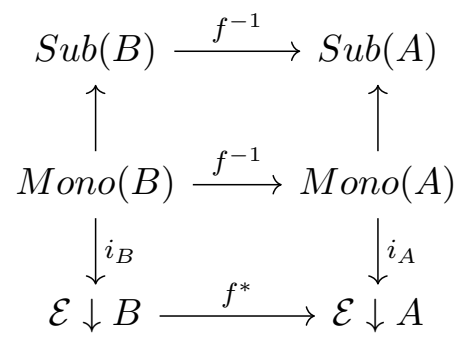

Esse $f^{*}$ é a que definimos em 2.4.2, o funtor mudança de base, logo, é um morfismo lógico e, portanto, pela observação 2.5.4, $f^{-1}$ é um homomorfismo de álgebras de Heyting.

Teorema 2.5.7 Seja $A$ um objeto de um topos $\mathcal{E}$. Então a potência $\Omega^{A}$ é uma Álgebra de Heyting interna. Em particular, o classificador de subobjeto, $\Omega$, também é. Ainda mais, essa estrutura é natural em $A$, no sentido que dado um morfismo $f: A \rightarrow B$ em $\mathcal{E}$, o mapa induzido $\Omega(f): \Omega^{B} \rightarrow \Omega^{A}$ é um homomorfismo de álgebras de Heyting internas.

\section{Demonstração:}

Vamos começar definindo o $\inf \wedge: \Omega^{A} \times \Omega^{A} \rightarrow \Omega^{A}$. Fixe $A$ um objeto de $\mathcal{E}$. Lembre-se que em um topos temos isomorfismos

$$
\begin{array}{r}
\operatorname{Sub}_{\mathcal{E}}(A \times X) \stackrel{\cong}{\rightarrow} \operatorname{Hom}(A \times X, \Omega) \stackrel{\cong}{\rightrightarrows} \operatorname{Hom}_{\mathcal{E}}\left(X, \Omega^{A}\right) \\
m: S \rightarrow A \times X \mapsto \chi_{m}: A \times X \rightarrow \Omega \mapsto \widetilde{\chi}_{m}: X \rightarrow \Omega^{A}
\end{array}
$$

para todo $X$ em $\mathcal{E}$. Considere a operação $\cap: S u b(A \times X) \times S u b(A \times X) \rightarrow S u b(A \times X)$, então existe $\bigwedge_{X}: \operatorname{Hom}\left(X, \Omega^{A} \times \Omega^{A}\right) \rightarrow \operatorname{Hom}\left(X, \Omega^{A}\right)$ natural em $X$ que faz o seguinte diagrama comutar:

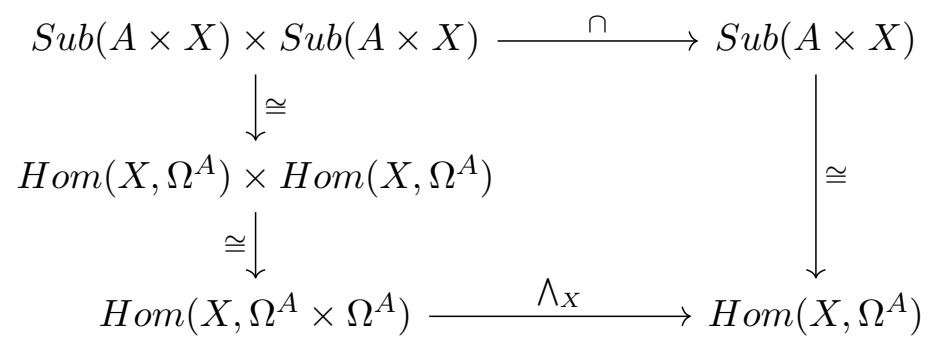

Considere $X=\Omega^{A} \times \Omega^{A}$ e aplique $\bigwedge_{X}$ na identidade, assim, pelo Lema de Yoneda, $\bigwedge_{X}$ é determinado de forma única por uma aplicação $\Lambda: \Omega^{A} \times \Omega^{A} \rightarrow \Omega^{A}$.

Definimos $\bigvee$ e $\Longrightarrow$ exatamente da mesma forma, bastaria trocar os símbolos. Para $\top$ e $\perp$ o procedimento é análogo.

Se $A=1$, então $\Omega^{A} \cong \Omega$ e vemos que o classificador de subobjeto é álgebra de Heyting interna.

Agora seja $f: A \rightarrow B$ morfismo em $\mathcal{E}$. Já vimos que para qualquer objeto $A$ em $\mathcal{E}$, $\operatorname{Sub}(A)$ tem estrutura de álgebra de Heyting. Levando em conta os isomorfismos, o seguinte diagrama comuta: 


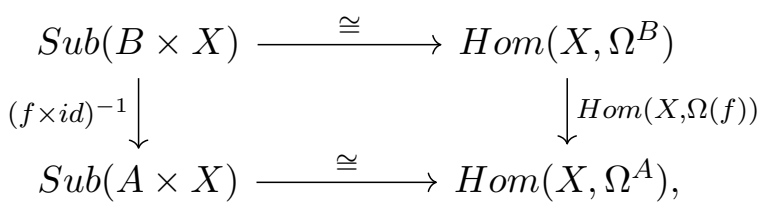

Como $(f \times i d)^{-1}$ é homomorfismo de álgebras de Heyting, Hom $(X, \Omega(f))$ também. Isso vale para todo objeto $X$ em $\mathcal{E}$, logo, pelo Lema de Yoneda, existe um único $\Omega(f): \Omega^{B} \rightarrow \Omega^{A}$ em $\mathcal{C}$ onde, por exemplo, o diagrama abaixo comuta.

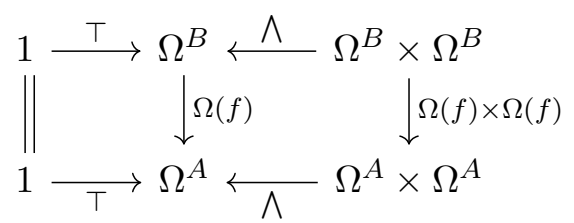

E conseguimos o mesmo para as outras aplicações necessárias. Concluímos que $\Omega(f)$ é um homomorfismo de Álgebras de Heyting internas.

Note que a estrutura interna de $\Omega^{A}$ torna $\operatorname{Hom}\left(X, \Omega^{A}\right)$ uma álgebra de Heyting externa para todo $X$ em $\mathcal{E}$, logo, os isomorfismos canônicos $\operatorname{Sub}_{\mathcal{E}}(A \times X) \cong \operatorname{Hom}(A \times X, \Omega) \cong$ $\operatorname{Hom}_{\mathcal{E}}\left(X, \Omega^{A}\right)$ são isomorfismos de álgebras de Heyting externas.

Aproveitando que nessa seção construímos o adjunto à esquerda $\exists_{f}$ do funtor $f^{-1}$ : $S u b(B) \rightarrow S u b(A)$, vamos comentar sobre a existência dos outros adjuntos. São eles: o adjunto à direita de $f^{-1}$, denotado por $\forall_{f}$ e os adjuntos internos à esquerda e à direita de $\Omega(f): \Omega^{B} \rightarrow \Omega^{A}$ denotados também por $\exists_{f}$ e $\forall_{f}$, respectivamente.

No meio do caminho precisaremos lançar mão da condição de Beck-Chevalley. Esse é um resultado técnico bastante conhecido demonstrado em diversos livros. Deixamos como referência [MLM94] (Capítulo IV - Seção 9 - Proposição 1).

Lema 2.5.8 Sejam $A, B$ e $C$ objetos em $\mathcal{E}$. Dado um pullback

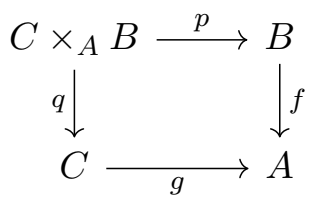

Temos que o seguinte diagrama comuta:

$$
\begin{array}{ccc}
\operatorname{Sub}\left(C \times_{A} B\right) & \stackrel{p^{-1}}{\leftrightarrows} & S u b(B) \\
\exists_{q} \downarrow \uparrow \uparrow_{q^{-1}} & & \exists_{f} \mid \uparrow \uparrow_{f^{-1}} \\
\operatorname{Sub}(C) \underset{g_{g}}{\stackrel{\exists^{-1}}{\leftrightarrows}} & \operatorname{Sub}(A)
\end{array}
$$

Em outras palavras, dado um subobjeto $U$ de $B$ e $V$ subobjeto de $C, g^{-1} \exists_{f} U=\exists_{q} p^{-1} U$ e $\exists_{p} q^{-1} V=f^{-1} \exists_{g} V$

Agora vamos tornar preciso o que queríamos dizer com "adjuntos internos". Sejam $(L, \leq)$ e $\left(L^{\prime}, \leq^{\prime}\right)$ ordens parciais internas (apresentamos o que isso significa logo após a Definição 2.5.3) em $\mathcal{E}$. Considere $L \underset{\phi}{\stackrel{\psi}{\leftrightarrows}} L^{\prime}$ onde $\phi$ e $\psi$ preservam ordem, ou seja, no caso de $\psi$ temos que a composição $\leq \longmapsto L \times L \stackrel{\psi \times \psi}{\longrightarrow} L^{\prime} \times L^{\prime}$ se fatora por $\leq^{\prime} \longmapsto L^{\prime} \times L^{\prime}$. Nessas condições:

Definição 2.5.9 Dizemos que $\phi$ é internamente adjunto à esquerda de $\psi$ se os morfismos $<\phi \psi, i d_{L^{\prime}}>\mathrm{e}<i d_{L}, \psi \phi>$ se fatoram, respectivamente, por $\leq^{\prime} \mathrm{e} \leq$, isto é, 

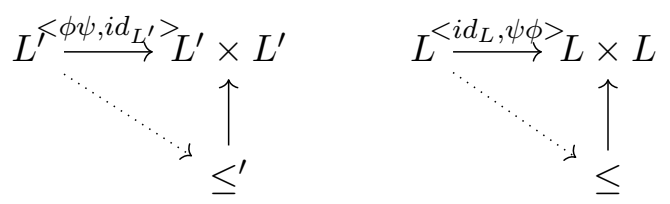

Note que dado $X$ objeto de $\mathcal{E}$, o conjunto $\operatorname{Hom}(X, L)$ tem ordem parcial induzida por todo par de morfismos $f, g$ em $\operatorname{Hom}(X, L)$

$$
f \leq g \text { se, e somente se, }(f, g): X \rightarrow L \times L \text { se fatora por } \leq \longmapsto L \times L .
$$

Desta forma conseguimos a seguinte definição equivalente.

Definição 2.5.10 Dizemos que $\phi: L \rightarrow L^{\prime}$ é internamente adjunto à esquerda de $\psi: L^{\prime} \rightarrow L$ se para todo objeto $X$ de $\mathcal{E}$ o mapa que preserva ordem $\phi_{*}: \operatorname{Hom}(X, L) \rightarrow$ $\operatorname{Hom}\left(X, L^{\prime}\right)$, induzido pela $\phi$ (através da composição), é adjunto à esquerda de $\psi_{*}$ : $\operatorname{Hom}\left(X, L^{\prime}\right) \rightarrow \operatorname{Hom}(X, L)$, induzido pela $\psi$.

Então temos que $\phi$ e $\psi$ são adjuntas internas se, e somente se, são adjuntas (no sentido usual) nos Hom's.

Analogamente definimos a noção de internamente adjunto à direita.

Teorema 2.5.11 Seja $f: A \rightarrow B$ em $\mathcal{E}$. Então $\Omega(f): \Omega^{B} \rightarrow \Omega^{A}$ possui adjunto à esquerda interno $\exists_{f}: \Omega^{A} \rightarrow \Omega^{B}$

\section{Demonstração:}

O Lema 2.5.6 nos fornece uma adjunção "externa", demonstrar a versão interna consiste basicamente de verificar que o resultado vale de forma natural. Vejamos: Seja $X$ um objeto em $\mathcal{E}$, temos um adjunto à esquerda (externo) $\exists_{(f \times i d)}$ de $(f \times i d)^{-1}$, ou seja,

$$
S u b_{\mathcal{E}}(A \times X) \underset{\exists_{(f \times i d)}}{\stackrel{(f \times i d)^{-1}}{\leftrightarrows}} \operatorname{Sub}(B \times X)
$$

Aplicando a condição de Beck-Chevalley (1.5.7) no caso particular de um pullback

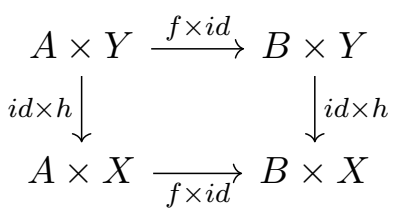

para qualquer $h: Y \rightarrow X$, obtemos o seguinte diagrama comutativo

$$
\begin{gathered}
\operatorname{Sub}(A \times Y) \stackrel{\exists_{(f \times i d)}}{\longrightarrow} \operatorname{Sub}(B \times Y) \\
(i d \times h)^{-1} \uparrow \\
\operatorname{Sub}_{(i d \times h)^{-1}}(A \times X) \underset{\exists_{(f \times i d)}}{\longrightarrow} \operatorname{Sub}(B \times X)
\end{gathered}
$$

Usando os isos naturais $\operatorname{Sub}_{\mathcal{E}}(A \times X) \cong \operatorname{Hom}_{\mathcal{E}}\left(X, \Omega^{A}\right)$ e $\operatorname{Sub}_{\mathcal{E}}(B \times X) \cong \operatorname{Hom}_{\mathcal{E}}\left(X, \Omega^{B}\right)$ obtemos únicos $\left(\left(\exists_{f}\right)_{X}\right)_{X}$ e $\left((\Omega(f))_{X}\right)_{X}$ naturais em $X$ de forma que

$$
\operatorname{Hom}_{\mathcal{E}}\left(X, \Omega^{A}\right) \underset{\left(\left(\exists_{f}\right)_{X}\right)_{X}}{\stackrel{\left.(\Omega(f))_{X}\right)_{X}}{\longrightarrow}} \operatorname{Hom}_{\mathcal{E}}\left(X, \Omega^{B}\right)
$$

Pelo Lema de Yoneda, $\left(\left(\exists_{f}\right)_{X}\right)_{X}$ e $\left((\Omega(f))_{X}\right)_{X}$ devem ser unicamente determinados por $\Omega^{A} \underset{\exists_{f}}{\stackrel{\Omega(f)}{\leftrightarrows}} \Omega^{B}$ e esse $\Omega(f)$ é precisamente o enunciado nesse teorema.

Como $(\star)$ é uma adjunção, temos que $\left(\exists_{f}\right)_{X}$ é adjunto à esquerda de $(\Omega(f))_{X}$ e esses morfismos são os induzidos por $\exists_{f}$ e $\Omega(f)$, respecivamente. Pela definição de adjunto interno concluímos que $\exists_{f}$ é adjunto interno à esquerda de $\Omega(f)$.

Também existem os adjuntos à direita, tanto o externo quanto o interno. 
Teorema 2.5.12 Seja $f: A \rightarrow B$ em $\mathcal{E}$. Então $f^{-1}: S u b(B) \rightarrow S u b(A)$ possui adjunto à direita $\forall_{f}: S u b(A) \rightarrow S u b(B)$

\section{Demonstração:}

Vamos nos aproveitar do seguinte diagrama comutativo que apareceu na demonstração do Teorema 2.5.7.

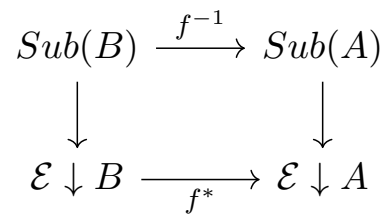

Pelo Teorema 2.4.9, o funtor mudança de base $f^{*}$ possui um adjunto à direita $\prod_{f}$. Como adjuntos à direita preservam objetos terminais e monomorfismos, temos que $f^{-1}$ é uma restrição de $f^{*}$ e assim conseguimos garantir que existe um funtor $\forall_{f}: S u b(A) \rightarrow \operatorname{Sub}(B)$ adjunto à direita de $f^{-1}$ que é uma restrição de $\prod_{f}$.

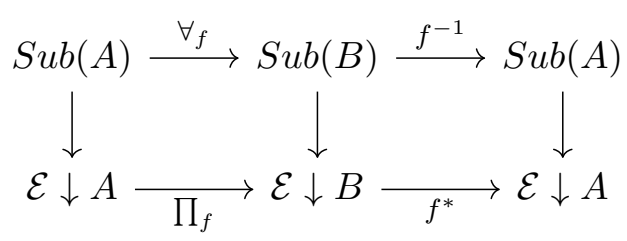

Teorema 2.5.13 Seja $f: A \rightarrow B$ em $\mathcal{E}$. Então $\Omega(f): \Omega^{B} \rightarrow \Omega^{A}$ possui adjunto interno à direita $\forall f: \Omega^{A} \rightarrow \Omega^{B}$.

\section{Demonstração:}

Basta "internalizar" a versão externa da mesma forma como procedemos no caso do $\exists_{f}$, pois para todo $h: Y \rightarrow X$, pela condição de Beck-Chevalley obtemos um diagrama comutativo

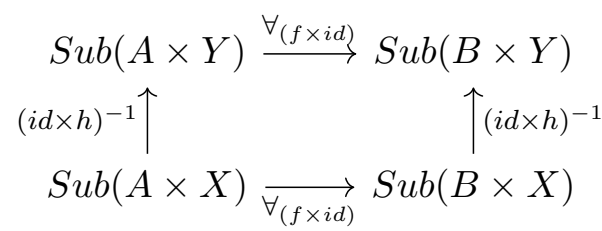

pois ele é equivalente ao diagrama dos adjuntos às esquerda - dado pela condição de Beck-Chevalley

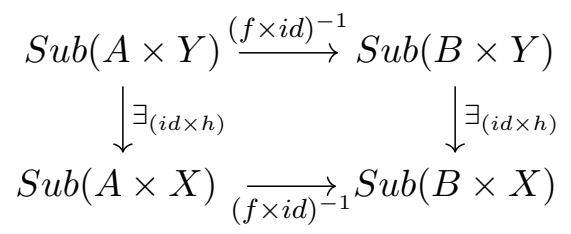

Pelo Lema de Yoneda e os isomorfismos naturais da forma naturais $S u b_{\mathcal{E}}(A \times X) \cong$ $\operatorname{Hom}_{\mathcal{E}}\left(X, \Omega^{A}\right)$ concluímos que existe um $\forall_{f}: \Omega^{A} \rightarrow \Omega^{B}$ adjunto interno à direita de $\Omega(f)$.

\subsection{Aspectos Lógicos da Teoria de Topos}

Dado um topos $\mathcal{E}$ podemos associar uma linguagem $L_{\mathcal{E}}$ onde os objetos passam a ser visto como conjuntos, os morfismos são simplesmente funções entre conjuntos, epimorfimos são sobrejeções e assim por diante, ou seja, conseguimos trabalhar como se estivéssemos em 
Sets, demonstrando resultados em topos como se estivéssemos em Sets. É nesse sentido que topos são considerados uma teoria de conjuntos generalizada.

A primeira pessoa a introduzir essa ideia foi W. Mitchell e vários matemáticos contribuiram para aperfeiçoar essa ideia que hoje é conhecida como linguagem de MitchellBénabou, a lógica interna de um topos. A definição que apresentamos está em em [Bor94c] e essa foi nossa referência ao longo dessa seção.

Ambas são definições longas, mas esperamos torná-las mais amigáveis com os comentários que faremos.

Definição 2.6.1 Seja $\mathcal{E}$ um topos. Escolha e fixe: um cone limite para cada diagrama; um morfismos vertade $t: 1 \longmapsto \Omega$; exponenciais. A linguagem de $\mathcal{E}$ consiste da listagem a seguir. Para todo objeto $A$ em $\mathcal{E}$ :

1. Para cada elemento $1 \rightarrow A$ de $A$ temos um símbolo chamado constante de tipo A

2. Um conjunto enumerável de símbolos chamado variável de tipo $A$.

Indutivamente definimos um termo, o qual é equipado com um tipo e variáveis livres.

3. Cada constante de tipo $A$ é um termo de tipo $A$ sem variáveis livres.

4. Cada variável $a$ de tipo $A$ é um termo de tipo $A$ onde $a$ é a única variável livre.

5. Se $\tau$ é um termo de tipo $A$ e $f: A \rightarrow B$ é um morfismo, a expressão $f(\tau)$ é um termo de tipo $B$ com as mesmas variáveis livres de $\tau$.

6. Se $\tau_{1}, \ldots, \tau_{n}$ são, respectivamente, termos de tipos $A_{1}, \ldots, A_{n}$, com as mesmas variáveis livres, então a expressão $\left(\tau_{1}, \ldots, \tau_{n}\right)$ é um termo de tipo $A_{1} \times \ldots \times A_{n}$ com as mesmas variáveis livres.

7. Se $\varphi$ é uma fórmula ${ }^{4}$ com variáveis livres $x_{1}, \ldots, x_{n}, y_{1}, \ldots, y_{m}$ de tipos $X_{1}, \ldots, X_{n}, Y_{1}$, $\ldots, Y_{m}$, com cada $x_{i}$ diferente de $y_{j}$, então a expressão $\left\{\left(x_{1}, \ldots, x_{n}\right) \mid \varphi\right\}$ é um termo de tipo $\Omega^{X_{1} \times X_{n}}$ com variáveis livres $y_{1}, \ldots, y_{m}$.

8. Se $\tau$ é um termo de tipo $A$ com variáveis livres $x_{1}, \ldots, x_{n}$ e se $x_{1}, \ldots, x_{m}$ é um conjunto de variáveis maior $(m \geq n)$, então a expressão $\tau_{<x_{1}, \ldots, x_{m}>}>$ é um termo de tipo $A$ com variáveis livres $x_{1}, \ldots, x_{m}$ e mantemos a notação dessa expressão apenas por $\tau$.

9. Seja $\tau$ um termo de tipo $A$ com variáveis livres $x_{1}, \ldots, x_{n}$ de tipo $X_{1}, \ldots, X_{n}$. Seja $\sigma_{1}, \ldots, \sigma_{n}$ termos de tipo $X_{1}, \ldots, X_{n}$ com as mesmas variáveis livres, sem ocorrência de limitador de $\tau$. Então a expresão $\tau\left(\sigma_{1}, \ldots, \sigma_{n}\right)$ é um termo de tipo $A$ com variáveis livres $\sigma_{1}, \ldots, \sigma_{n}$.

Agora definimos indutivamente uma fórmula, a qual é equipada com um conjunto de variáveis livres.

10. Os símbolos "verdadeiro"e "falso"são fórmulas sem variáveis livres.

11. Se $\tau$ e $\sigma$ são termos de tipo $A$ com as mesmas variáveis livres, então a expressão $\tau=\sigma$ é uma fórmula com as mesmas variáveis livres.

12. Se $\tau$ e $\sigma$ são termos de tipo $A$ e $\Sigma$ é um termo de tipo $\Omega^{A}$, todos com as mesmas variáveis livres, então a expressão $\tau \in \Sigma$ é uma fórmula com as mesmas variáveis livres.

13. Se $\varphi$ é uma fórmula, então a expressão $\neg \varphi$ é uma fórmula com as mesmas variáveis livres.

\footnotetext{
${ }^{4}$ Fórmulas são termos de tipo $\Omega$.
} 
14. Se $\varphi$ e $\psi$ são fórmulas com as mesmas variáveis livres, então as expressões $\varphi \bigwedge \psi$, $\varphi \bigvee \psi$ e $\varphi \Longrightarrow \psi$ são fórmulas com as mesmas variáveis livres.

15. Se $\varphi$ é uma fórmula com variáveis livres $x, y_{1}, \ldots, y_{n}$, onde $x$ é diferente de cada $y_{i}$, então as expressões $\exists x \varphi$ e $\forall x \varphi$ são fórmulas com variáveis livres $y_{1}, \ldots, y_{m}$.

16. Se $\varphi$ é uma fórmula com variáveis livres $x_{1}, \ldots, x_{n}$ e se $x_{1}, \ldots, x_{m}$ é um conjunto de variáveis maior $(m \geq n)$, então a expressão $\varphi_{\left.<x_{1}, \ldots, x_{m}\right\rangle}$ é uma fórmula com variáveis livres $x_{1}, \ldots, x_{m}$ e mantemos a notação dessa expressão apenas por $\varphi$.

17. Seja $\varphi$ é uma fórmula com variáveis livres $x_{1}, \ldots, x_{n}$ de tipo $X_{1}, \ldots, X_{n}$. Sejam $\sigma_{1}, \ldots, \sigma_{n}$ termos de tipo $X_{1}, \ldots, X_{n}$ com as mesmas variáveis livres, sem ocorrência de limitador de variável da fórmula $\varphi$, então $\varphi\left(\sigma_{1}, \ldots, \sigma_{n}\right)$ é uma fórmula com as variáveis livres $\sigma_{1}, \ldots, \sigma_{n}$.

Primeiro, o que queremos dizer por variáveis livres e com limitadores nesse contexto? Uma ocorrência de variável é livre quando esta não aparece no escopo de nenhum limitador de variável. Vamos buscar entender através de um exemplo. Imagine $A=\mathbb{N}$, temos que $2 x$ é um termo de tipo $\mathbb{N}$ e $x$ é variável livre. Por outro lado, algo da forma $3 \sum_{x=0}^{4} 2 x+5$ é um termo de tipo $\mathbb{N}$, mas a variável $x$ é limitada pelo operador $\sum_{x=0}^{4}$. Note que o valor de $2 x$ depende de $x$, mas o valor de $3 \sum_{x=0}^{4} 2 x+5$ é fixo, no caso, 65 .

Pode parecer que não estamos fazendo nada de novo, os símbolos usados são os mesmo que aparecem quando estamos em Sets, a questão é o que eles significam. Por exemplo se temos $a$ e $b$ variáveis de tipo $A$, na categoria nos conjuntos sabemos o que significa $a=b$ (caso ainda esteja muito geral, tome $A=\mathbb{N}$ ). O próximo passo é dizer como atribuir significado a esses símbolos quando estamos em um topos, chamaremos isso de "interpretação".

Definição 2.6.2 Seja $\mathcal{E}$ um topos. A interpretação de $\mathcal{E}$ consiste da listagem a seguir:

1. Para cada termo $\tau$ de tipo $A$ com variáveis $x_{1}, \ldots, x_{n}$ de tipos $X_{1}, \ldots, X_{n}$, especificamos sua realização por $\ulcorner\tau\urcorner: X_{1} \times \ldots \times X_{n} \rightarrow A$.

2. Para cada fórmula $\varphi$ com variáveis livres $x_{1}, \ldots, x_{n}$ de tipos $X_{1}, \ldots, X_{n}$ especificamos uma tabela verdade $\ulcorner\varphi\urcorner: X_{1} \times \ldots \times X_{n} \rightarrow \Omega$.

Dada a realização $\ulcorner\tau\urcorner$ de $\tau$ prosseguimos indutivamente:

3. Uma variável de tipo $A$ admite como realização o morfismo identidade de $A$.

4. Se $k: 1 \rightarrow A$ é elemento global de $A$, então a correspondente constante de tipos $A$ admite o próprio morfismo $k$ como realização.

5. Se $\tau$ é um termo de tipo $A$ e $f: A \rightarrow B$ é um morfismo, então a expressão $f(\tau)$ admite uma realização $f \circ\ulcorner\tau\urcorner$

6. Se $\tau_{1}, \ldots, \tau_{n}$ são, respectivamente, termos de tipos $A_{1}, \ldots, A_{n}$, com as mesmas variáveis livres $x_{1}, \ldots, x_{m}$ de tipos $X_{1}, \ldots, X_{m}$, então a realização do termo $\left(\tau_{1}, \ldots, \tau_{n}\right)$ é um morfismo $\left(\left\ulcorner\tau_{1}\right\urcorner, \ldots,\left\ulcorner\tau_{n}\right\urcorner\right): X_{1} \times \ldots \times X_{m} \rightarrow A_{1} \times \ldots \times A_{n}$

7. Se $\varphi$ é uma fórmula com variáveis livres $x_{1}, \ldots, x_{n}, y_{1}, \ldots, y_{m}$ de tipos $X_{1}, \ldots, X_{n}, Y_{1}$, $\ldots, Y_{m}$, com cada $x_{i}$ diferente de $y_{j}$, então a realização do termo $\left\{\left(x_{1}, \ldots, x_{n}\right) \mid \varphi\right\}$ é o morfismo $Y_{1} \times \ldots \times Y_{m} \rightarrow \Omega^{X_{1} \times \ldots \times X_{n}}$, que corresponde à $\ulcorner\varphi\urcorner$ pela propriedade do topos de ser categoria cartesianamente fechada, isto é, o morfismo de transposta exponencial. 
8. Se $x_{1}, \ldots, x_{m}$ são variáveis livres de tipos $X_{1}, \ldots, X_{m}$ e $\tau$ um termo de tipo $A$ com variáveis livres, onde $m \geq n$, então a realização do termo $\tau_{<x_{1}, \ldots, x_{m}>}$ é a composição $X_{1} \times \ldots \times X_{m} \stackrel{\pi}{\rightarrow} X_{1} \times \ldots \times X_{n} \stackrel{\ulcorner\tau\urcorner}{\longrightarrow} A$, com $\pi$ a projeção canônica.

9. Seja $\tau$ um termo de tipo $A$ com variáveis livres $x_{1}, \ldots, x_{n}$ de tipo $X_{1}, \ldots, X_{n}$. Seja $\sigma_{1}, \ldots, \sigma_{n}$ termos de tipo $X_{1}, \ldots, X_{n}$ com as variáveis livres $y_{1}, \ldots, y_{m}$ de tipo $Y_{1}, \ldots, Y_{m}$. Suponha que nenhum limitador de variável de $\tau$ apareça entre as variáveis $\sigma_{1}, \ldots, \sigma_{n}$ . Então a realização do termo $\tau\left(\sigma_{1}, \ldots, \sigma_{n}\right)$ é a composição $Y_{1} \times \ldots \times Y_{m} \stackrel{\left(\left\ulcorner\tau_{1}\right\urcorner, \ldots,\left\ulcorner\tau_{m}\right\urcorner\right)}{\longrightarrow}$ $X_{1} \times \ldots \times X_{n} \stackrel{\ulcorner\tau\urcorner}{\longrightarrow} A$.

Analogamente procedemos com a tabela verdade de uma fórmula:

10. A tabela verdade da "verdade" é o morfismo $t: 1 \rightarrow \Omega$ e a do falso é $f: 1 \rightarrow \Omega$, onde $f$ é o morfismo característico de $0 \longmapsto A$, isto é, $f$ é o único morfismo em $\mathcal{E}$ tal que<smiles>CCCCCCCCCO</smiles>

11. Se $\tau$ e $\sigma$ são termos de tipo $A$ com as mesmas variáveis livres $x_{1}, \ldots, x_{n}$ de tipo $X_{1}, \ldots, X_{n}$, então a fórmula $\tau=\sigma$ admite como tabela verdade o morfismo $X_{1} \times$ $\ldots \times X_{n} \stackrel{(\ulcorner\tau\urcorner,\ulcorner\sigma\urcorner))}{\longrightarrow} A \times A \stackrel{=_{A}}{\longrightarrow} \Omega$, onde $={ }_{A}$ é o morfismo característico da diagonal $\triangle_{A}: A \longmapsto A \times A$

12. Se $\tau$ é um termo de tipo $A$ e $\Sigma$ é um termo de tipo $\Omega^{A}$, ambos com as mesmas variáveis livres $x_{1}, \ldots, x_{n}$ de tipo $X_{1}, \ldots, X_{n}$, então a fórmula $\tau \in \Sigma$ admite tabela verdade $X_{1} \times \ldots \times X_{n} \stackrel{(\ulcorner\tau\urcorner,\ulcorner\Sigma\urcorner)}{\longrightarrow} A \times \Omega^{A} \stackrel{\epsilon_{A}}{\longrightarrow} \Omega$ onde $\epsilon_{A}$ é o transposta exponencial da identidade $i d_{\Omega^{A}}: \Omega^{A} \longmapsto \Omega^{A}$

13. Se $\varphi$ é uma fórmula com variáveis livres $x_{1}, \ldots, x_{n}$ de tipo $X_{1}, \ldots, X_{n}$, então a fórmula $\neg \varphi$ admite tabela verdade $X_{1} \times \ldots \times X_{n} \stackrel{\ulcorner\varphi\urcorner}{\longrightarrow} \Omega \stackrel{\ulcorner\neg\urcorner}{\longrightarrow} \Omega$

14. Se $\varphi$ e $\psi$ são fórmulas com as mesmas variáveis livres $x_{1}, \ldots, x_{n}$ de tipo $X_{1}, \ldots, X_{n}$, então as fórmulas $\varphi \bigwedge \psi, \varphi \bigvee \psi$ e $\varphi \Longrightarrow \psi$ admitem, respectivamente, as seguintes tabelas verdade

$$
\begin{aligned}
& X_{1} \times \ldots \times X_{n} \stackrel{(\ulcorner\varphi\urcorner,\ulcorner\psi\urcorner)}{\longrightarrow} \Omega \times \Omega \stackrel{\wedge}{\longrightarrow} \Omega \\
& X_{1} \times \ldots \times X_{n} \stackrel{(\ulcorner\varphi\urcorner,\ulcorner\psi\urcorner)}{\longrightarrow} \Omega \times \Omega \stackrel{\vee}{\longrightarrow} \Omega \\
& X_{1} \times \ldots \times X_{n} \stackrel{\ulcorner\varphi\urcorner,\ulcorner\psi\urcorner)}{\longrightarrow} \Omega \times \Omega \stackrel{\Longrightarrow}{\longrightarrow} \Omega
\end{aligned}
$$

15. Se $\varphi$ é uma fórmula com variáveis livres $x, y_{1}, \ldots, y_{n}$ de tipo $X, Y_{1}, \ldots, Y_{n}$, onde $x$ é diferente de cada $y_{i}$, e se $S \longmapsto X \times Y_{1} \times \ldots \times Y_{n}$ é subobjeto classificado por $\ulcorner\varphi\urcorner$, então as fórmulas $\exists x \varphi$ e $\forall x \varphi$ admitem como tabela verdade, respectivamente os morfismos característicos dos subobjetos $\exists_{\pi}(S)$ e $\forall_{\pi}(S)$ de $Y_{1} \times \ldots \times Y_{n}$, onde $\pi$ é a projeção canônica.

16. Se $x_{1}, \ldots, x_{m}$ são variáveis livres de tipo $X_{1}, \ldots, X_{m}$ e $\varphi$ é uma fórmula com variáveis livres $x_{1}, \ldots, x_{n}$ com $m \geq n$, então a fórmula $\varphi_{<x_{1}, \ldots, x_{m}>}$ admite como tabela verdade a composição $X_{1} \times \ldots \times X_{m} \stackrel{\pi}{\rightarrow} X_{1} \times \ldots \times X_{n} \stackrel{\ulcorner\varphi\urcorner}{\longrightarrow} \Omega$ onde $\pi$ é a projeção canônica. 
17. Seja $\varphi$ é uma fórmula com variáveis livres $x_{1}, \ldots, x_{n}$ de tipo $X_{1}, \ldots, X_{n}$. Sejam $\sigma_{1}, \ldots, \sigma_{n}$ termos de tipo $X_{1}, \ldots, X_{n}$ com as mesmas variáveis livres $y_{1}, \ldots, y_{m}$ de tipo $Y_{1}, \ldots, Y_{m}$. Suponha que nenhum limitador de variável de $\varphi$ apareça entre as variáveis $\sigma_{1}, \ldots, \sigma_{n}$, então a fórmula $\varphi\left(\sigma_{1}, \ldots, \sigma_{n}\right)$ admite tabela verdade

$$
Y_{1} \times \ldots \times Y_{n} \stackrel{\left(\left\ulcorner\sigma_{1}\right\urcorner, \ldots,\left\ulcorner\sigma_{n}\right\urcorner\right)}{\longrightarrow} X_{1} \times \ldots \times X_{m} \stackrel{\ulcorner\varphi\urcorner}{\longrightarrow} \Omega
$$

Para ficar mais claro o que está acontecendo no item 15 deixamos o diagrama de $\exists_{\pi}(S)$.

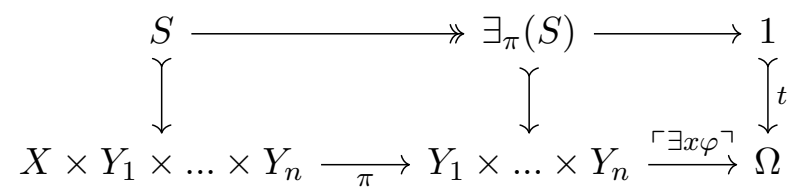

Escolhemos apresentar dessa forma o que é uma linguagem e o que é a sua respectiva interpretação pois assim parece ficar mais claro o procedimento. Ao definir linguagem começamos dizendo o que é uma constante e o que é uma variável. Usamos esses dois conceitos para ir definindo o que são termos, isto é, tudo aquilo que se encaixa na listagem de 3 à 9 de definição 2.7.1. Depois usamos de 10 à 17 para dizer exatamente o que são fórmulas. Uma linguagem é algo que consiste de termos e fórmulas, uma vez definidos constate e variável.

Procedemos de forma análoga para definir a interpretação de linguagem. Dizemos o que são realizações - a partir disso como é a realização de cada termo - e tabelas verdade - a partir disso como é a tabela verdade de cada fórmula.

Agora que temos fórmulas e tabelas verdade uma pergunta natural é: como decidimos que uma fórmula é válida?

Definição 2.6.3 Em um topos, seja $\varphi$ uma fórmula com variáveis livres $x_{1}, \ldots, x_{n}$ de tipo $X_{1}, \ldots, X_{n}$. Dizemos que $\varphi$ é válida quando a tabela verdade de $\varphi$ é a composição

$$
X_{1} \times \ldots \times X_{n} \stackrel{!}{\rightarrow} 1 \stackrel{t}{\rightarrow} \Omega .
$$

Isto é, $t_{X_{1} \times \ldots \times X_{n}}$. Notação: $\models \varphi$

Vamos dar um exemplo simples de fórmula válida num topos.

Exemplo 2.6.4 $\mathcal{E} \models x={ }_{X} x$

Nesse caso a nossa fórmula é $x={ }_{X} x$. A tabela verdade, pelo item 11 é $X \stackrel{(\ulcorner x\urcorner,\ulcorner x\urcorner)}{\longrightarrow}$ $X \times X \stackrel{=}{\longrightarrow} \Omega$. Como $\ulcorner x\urcorner=i d_{X}: X \rightarrow X$, temos $(\ulcorner x\urcorner,\ulcorner x\urcorner)=\triangle_{X}$. Uma vez que $=_{X}$ é morfismo característico da diagonal $\triangle_{X}$, o diagrama abaixo é um pullback

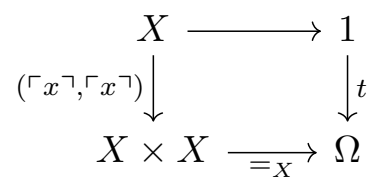

Em particular, o diagrama comuta, $\log$ o, $X \rightarrow 1 \stackrel{t}{\rightarrow} \Omega$ é tabela verdade, como queríamos.

Podemos expressar características de um morfismo como a injetividade e a sobrejetividade através de fórmulas, as quais serão válidas em um topos se, e só se, o morfismo for, respectivamente, mono e epi. Usando a notação $a: A$ para dizer que $a$ é de tipo $A$ :

Fato 2.6.5 Seja $g: A \rightarrow B$ morfismo em $\mathcal{E}$.

1. $g$ é mono se, e somente se,

$$
g a=g a^{\prime} \vdash a=a^{\prime} \quad a: A, a^{\prime}: A
$$


2. $g$ é epi se, e somente se,

$$
\vdash(\exists a: A) b=g a \quad b: B
$$

Assim, $g$ é isomorfismo se, e somente se, valem as expressões da inejetividade e sobrejetividade simultaneamente. A prova desse fato pode ser encontrada em [McL96], Seção 16.2 .

Apresentamos as principais definições e apresentamos um exemplo, agora queremos apenas tecer alguns comentários sobre o papel da Lógica na Teoria de Topos.

Para os mais próximos da Lógica, não deve ser difícil ver que essa descrição da linguagem de um Topos fornece uma lógica de primeira ordem. Isso era de ser esperar dada a forte relação do topos com as Álgebras de Heyting, como vimos no Teorema 2.5.6, se $f: A \rightarrow B$ é um morfismo em um topos, temos que $S u b(A), S u b(B)$ possuem estrutura de Álgebra de Heyting e $f^{-1}: S u b(B) \rightarrow S u b(A)$ é um homomorfismo de álgebras de Heyting. ${ }^{5}$ Todos esses fatores nos levam a crer que topos satisfazem o cálculo proposicional intuicionista, de fato satisfazem, veja o Teorema 6.7.1 de [Bor94c], por exemplo. Todavia, apesar de Sets ser um exemplo de topos, nem sempre lei do terceiro excluído não vale em um topos, desta forma, a lógica clássica não vale em geral.

Portanto, quando dizemos que um topos, internamente, se parece com a categoria de conjutos estamos dizendo que se temos uma construção em Sets que utiliza apenas a lógica intuicionista, então podemos copiar os mesmos argumentos em um topos $\mathcal{E}$.

Para isso ficar claro e com todos os detalhes formais recomendamos o capítulo 6 de [Bor94c], mas podemos dar um exemplo concreto que deve facilitar a compreensão do que estamos querendo dizer.

Seja $G$ um grupo com uma operação $m$ cujo elemento identidade é denotado por $e$. Sabemos que o elemento inverso de um grupo é único e podemos escrever isso usando apenas símbolos e fórmulas, de forma que a expressão faz sentido na linguagem interna do topos:

$$
a: G, b: G, c: G \mid m(a, b)=e \vee m(a, c)=e \vdash b=c
$$

Agora imagine que em vez de tomarmos $G$ um grupo, quisessemos que $G$ fosse um objeto de alguma categoria $\mathcal{C}$. Como definir isso? O elemento inverso ainda é único? Aliás, como é o elemento inverso? Detalhe, queremos preservar a noção usal de grupo, ou seja, ainda queremos que $G$, como objeto de $\mathcal{C}$, seja uma espécie de "conjunto generalizado" munido com uma operação e satisfazendo os já conhecidos axiomas.

Definição 2.6.6 Seja $\mathcal{C}$ uma categoria com produtos binários e objeto terminal denotado por 1. Um objeto de grupo em $\mathcal{C}$ é um objeto $G$ de $\mathcal{C}$ com morfismos em $\mathcal{C}$

$$
e: 1 \longrightarrow G \quad i: G \longrightarrow G \quad m: G \times G \longrightarrow G
$$

de forma que os seguintes diagramas comutam

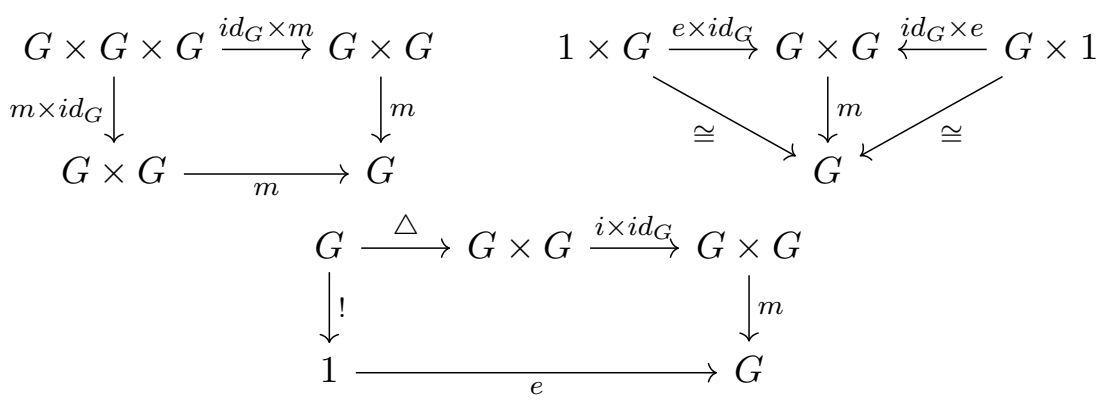

\footnotetext{
${ }^{5} \mathrm{Na}$ verdade, um topos é uma categoria de Heyting, mas definir essa categoria vai além do nosso interesse nesse trabalho. Deixamos como referência [Joh02b].
} 
O morfismo $\triangle: G \rightarrow G \times G$ é o morfismo diagonal - quando composto com a projeção, dá a identidade.

Se olharmos cuidadosamente para esses diagramas veremos que eles indicam os axiomas de grupo, por exemplo, o primeiro indica a associatidade.

Resumindo, um objeto de grupo é uma quádrupla $(G, e, i, m)$ em que os três diagramas acima comutam.

Observação 2.6.7 Objetos de grupo são bem conhecidos apesar de normalmente não serem apresentados dessa forma, alguns exemplos notáveis são os grupos topológicos e os grupos de Lie, os quais surgem quando estamos, respectivamente, na categoria Top, dos espaços topológicos, e Man, das variedades suaves.

Então o elemento identidade de um grupo em uma categoria $\mathcal{C}$ é o morfismo $e$. Além disso, é possível demonstrar a expressão $(\star)$ usando apenas recursos da lógica da categoria dos grupos abelianos (é conhecida como lógica regular, como a lógica associada às categorias regulares. Toda categoria abeliana é regular - Exemplo 2.4.1 de [Bor94b] - e topos também são categorias regulares - Corolário 5.9.2 de [Bor94b]. Não vamos nos estender sobre isso, mas as semelhanças entre topos e categorias abelianas ocorrem por ambas serem categorias regulares, listamos exemplos de regularidade no Capítulo 4 quando comparamos essas duas categorias). Isso significa que a expressão é válida não apenas para grupos, mas grupos definidos em qualquer categoria.

Então bastaria provar o resultado apenas uma vez para ver que ele vale em diversas outras ocasiões, mas o preço a pagar por essa facilidade é se restringir a uma certa lógica, nesse exemplo a regular, ao executar a verificação.

Usamos esse exemplo não apenas porque a teoria de grupo é equacionalmente simples, mas também porque será de extrema importância no próximo capítulo. 


\section{Capítulo 3}

\section{Álgebra Homológica em Topos}

Em Álgebra Homológica usamos categorias abelianas exaustivamente. Uma vez que desejamos estudar Álgebra Homológica em topos e tendo em vista as inúmeras propriedades em comum entre categorias abelianas e topos, a pergunta mais natural a ser feita é: todo topos pode ter seus hom-sets enriquecidos naturalmente em grupos abelianos de forma que a categoria resultante seja uma categoria abeliana?

Se esse fosse o caso não teríamos nada com o que nos preocuparmos e a categogra dos topos se encaixaria perfeitamente no que apresentamos, porém, topos são categorias cartesianamente fechadas enquanto categorias abelianas possuem zero objeto, mas nenhuma categoria cartesianamente fechada (não trivial) possui zero objeto. Com efeito, seja $X$ objeto do topos $\mathcal{E}$, supondo que é também categoria abeliana temos que os objetos terminal e inicial coincidem, isto é, $1=0$, então $X \cong X^{1} \cong X^{0} \cong 1$. Isso nos diz que todo objeto $X$ é terminal, assim $\mathcal{E}$ é equivalente a uma categoria com um único elemento.

Para darmos sentido ao estudo da Álgebra Homológica em um topos vamos introduzir a categoria dos grupos abelianos (internos) em um topos $\mathbf{A b}(\mathcal{E})$, esta é categoria abeliana para qualquer topos - usando a noção de objeto de grupo da Definição 2.6.6 - pois quando $\mathcal{E}$ é um topos de Grothendieck, $\mathbf{A b}(\mathcal{E})$ é categoria abeliana e, mais do que isso, possui suficientes injetivos, assim podemos construir funtores derivados à direita e definir grupo de cohomologia em um topos.

Esse capítulo é o grande objetivo desse trabalho de Mestrado, onde reunimos as ferramentas de álgebra homológica e de teoria de topos. Vamos ser mais explícitos em algumas passagens dos resultados apresentados em [Joh14] e fornecer uma segunda descrição da categoria dos grupos abelianos em um topos - já conhecida na literatura, mas não considerada na referência citada.

Entendido que topos de Grothendieck são uma generalização de feixes de conjuntos é de se esperar que o estudo da cohomologia nesse espaço mais abstrato se comporte de forma similiar ao caso já conhecido. Com a finalidade de evidenciar as semelhanças começamos introduzindo a usual cohomologia de feixes e para isso usamos [Ten75] e [Ive84].

\subsection{Feixes Algébricos}

Comentamos acima que topos não são (e não podem ser enriquedidos para serem) categorias abelianas, o que naturalmente inclui Sets. Assim, feixes de conjuntos, como funtores que levam em Sets, também não podem ser enriquecidos de forma que resultem em uma categoria abeliana.

Contornamos isso da mesma forma como faremos para topos, usando objetos de grupo abeliano, essa abordagem é feita em [MLM94], mas a demonstrações dessa seção estão em grande parte em [Ive84], as exceções são as Proposições 3.1.5 e 3.1.6, são resultados bem conhecidos mas que costumam ser deixadas como exercício para o leitor e abrimos as contas aqui.

Vamos adicionar à Definição 2.6.6 o diagrama comutativo 


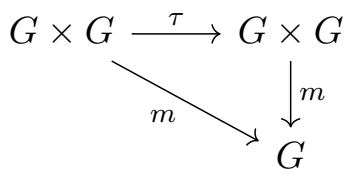

onde, $\tau: G \times G \rightarrow G \times G$ é o morfismo que inverte a posição das coordenadas.

Esse diagrama representa o axioma adicional para grupos abelianos.

Definição 3.1.1 Seja $\mathcal{C}$ categoria com objeto terminal e produtos binários. Definimos $\operatorname{Ab}(\mathcal{C})$ como a categoria cujos objetos são objetos de grupo abeliano em $\mathcal{C}$ e os morfismos são morfismos em $\mathcal{C}$ que comutam com os mapas $e, i$ e $m$.

Sendo mais explícitos sobre esses morfismos, se temos $f:(G, e, i, m) \rightarrow\left(G^{\prime}, e^{\prime}, i^{\prime}, m^{\prime}\right)$, os seguintes diagramas comutam:
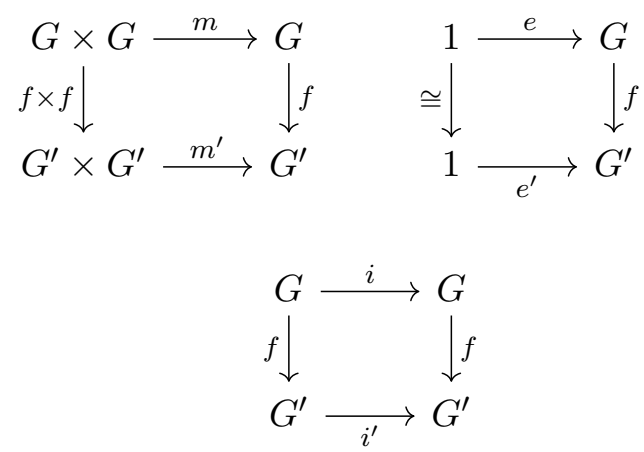

Se $\mathcal{C}=S e t s$, então teríamos $\mathbf{A b}(\mathcal{C}) \simeq \mathbf{A b}$ a própria categoria dos grupos abelianos.

Como um topos $\mathcal{E}$ é categoria que possui produtos binários e objeto terminal, podemos definir $\mathbf{A b}(\mathcal{E})$ sem problema.

Ainda, é possível caracterizar $\mathbf{A b}(\mathcal{E})$ como um modelo de um esboço $S_{A b}$ de forma que $\operatorname{Ab}(\mathcal{E}) \simeq \operatorname{Mod}\left(S_{A b}, \mathcal{E}\right)$ ou como um modelo de uma teoria algébrica, para um entendimento de como isso pode ser feito no caso dos esboços indicamos [BW85]; para modelos, [Joh02b].

Agora podemos definir:

Definição 3.1.2 Seja $X$ um espaço topológico. Um pré-feixe de grupos abelianos sobre $X$ é um objeto de grupo abeliano de $\operatorname{Sets}^{\mathcal{O}(X)^{o p}}$

Assim os morfismos $e_{U}: 1 \rightarrow \mathscr{F}(U), i_{U}: \mathscr{F}(U) \rightarrow \mathscr{F}(U)$ e $m_{U}:(\mathscr{F} \times \mathscr{F})(U)=$ $\mathscr{F}(U) \times \mathscr{F}(U) \rightarrow \mathscr{F}(U)$ são transformações naturais em $U$ aberto de $X$ que tornam comutativo os diagramas acima. Ainda, $\mathscr{F} \times \mathscr{F}$ em $\operatorname{Sets}^{\mathcal{O}(X)^{o p}}$ calculado ponto a ponto é um produto em Sets e temos um isomorfismo $(\mathscr{F} \times \mathscr{F})(U) \cong \mathscr{F}(U) \times \mathscr{F}(U)$, dessa forma as transformações naturais $m_{U}, e_{U}, i_{U}$ nos dizem que $\mathscr{F}(U)$ é um grupo abeliano, ou seja, temos um funtor $\mathscr{F}: \mathcal{O}(X)^{o p} \rightarrow A b$. Reciprocamente, se tormarmos $\mathscr{F} \in A b^{\mathcal{O}(X)^{o p}}$, como $\mathscr{F}(U)$ é grupo abeliano, temos morfismos $m_{U}, e_{U}, i_{U}$ que satisfazem as leis diagramaticais de objeto de grupo abeliano e, portanto, $\mathscr{F} \in \mathbf{A b}\left(\operatorname{Sets}^{\mathcal{O}(X)^{o p}}\right)$. Com isso descrevemos uma equivalência de categorias $\mathbf{A b}\left(\operatorname{Sets} s^{\mathcal{O}(X)^{o p}}\right) \simeq A b^{\mathcal{O}(X)^{o p}}$.

Podemos tomar também grupos abelianos em $S h(X)$ :

Definição 3.1.3 Um feixe de grupos abelianos sobre $X$ é um objeto de grupo abeliano de $\operatorname{Sh}(X)$.

Passaremos a deixar o "abeliano" implícito, usando apenas "grupo" para nos referirmos ao "grupo abeliano"e $X$ sempre será um espaço topológico.

Isso significa que se tomarmos $\mathscr{F}$ feixe de grupos então a composição $\mathcal{O}(X)^{o p} \stackrel{\mathscr{F}}{\longrightarrow}$ $\mathrm{Ab}($ Sets $) \stackrel{E}{\rightarrow}$ Sets, onde $E$ é o funtor esquecimento, é um feixe no sentido da Definição 2.1.2. 
A equivalência $\mathbf{A b}\left(\operatorname{Sets}^{\mathcal{O}(X)^{o p}}\right) \simeq A b^{\mathcal{O}(X)^{o p}}$ induz $S h_{A b}(X) \simeq \mathbf{A b}(S h(X))$, onde denotamos $S h_{A b}(X)$ como a categoria dos feixes de grupos $\mathscr{F}: \mathcal{O}(X)^{o p} \rightarrow A b$.

Comentamos ao fim da seção 2.1 sobre uma equivalência de categorias do tipo

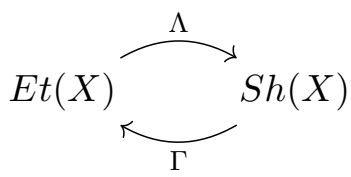

Essa equivalência se repete para o caso algébrico.

Definição 3.1.4 Um fibrado étale grupo abeliano é um objeto de grupo de $E t(X)$.

Assim como os fibrados étale nos davam uma noção geométrica de feixe, agora os fibrados étale grupo abeliano nos dão uma noção geométrica de feixe de grupo. Denotaremos a categoria por $E t_{A b}(X) \simeq \mathbf{A b}(E t(X))$ e é possível encontrar outra equivalência de categorias do tipo

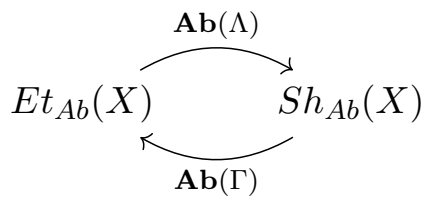

onde $\mathbf{A b}(\Lambda)$ e $\mathbf{A b}(\Gamma)$ são funtores induzidos por $\Lambda$ e $\Gamma$. Essas equivalências entram como uma curiosidade nesse trabalho, mas detalhes podem ser encontrados em [MLM94] e [Ten75]. Nosso real objetivo com essa seção é trazer uma versão de categoria de feixes em que podemos fazer Álgebra Homológica, ou seja, uma versão que seja categoria abeliana.

Sejam $\mathscr{F}, \mathscr{G}$ e $\mathscr{H}$ pré-feixes de grupos de $X$. Uma vez que a soma de morfismos entre feixes (que são transformações naturais) é dada por $(f+g)(U)=f(U)+g(U)$ e a composição é $(h \circ f)(U)=h(U) \circ f(U)$, para cada $U$ aberto de $X$ é imediato que a categoria dos pré-feixes de grupos é pré-aditiva.

Verificar que é aditiva também é extremamente simples, basta considerar $(\mathscr{F} \oplus \mathscr{G})(U)=$ $\mathscr{F}(U) \oplus \mathscr{G}(U)$ e é claro que isso é um pré-feixe abeliano. O terminal (e portanto nulo pela Proposição 1.1.9) é o funtor constante (pré-feixe constante) denotado por 1 tal que $1(U)=$ $\{*\}$ para todo aberto $U$, onde $\{*\}$ é o grupo trivial, pois $O(X)^{o p} \stackrel{1}{\longrightarrow}$ Ab $\stackrel{E}{\longrightarrow}$ Sets , $E(1(U))=E(\{*\})=\{*\}$ e $\{*\}$ é terminal em Sets.

Proposição 3.1.5 A categoria dos pré-feixes abelianos $A b^{\mathcal{O}(X)^{o p}}$ é abeliana.

\section{Demonstração:}

Seja $f: \mathscr{F} \rightarrow \mathscr{G}$ morfismo de pré-feixes. Defina

$$
(\operatorname{Ker} f)(U)=\operatorname{Ker}(f(U)) \text { e }(\operatorname{Coker} f)(U)=\operatorname{Coker}(f(U))
$$

Uma vez que $f(U) \in \mathscr{G}(U)$ e $\mathscr{G}(U)$ tem estrutura de grupo abeliano, é imediato que o kernel e o cokernel existem. Também não é difícil ver que assim definidos são pré-feixes.

Recorde que $f(U): \mathscr{F}(U) \rightarrow \mathscr{G}(U)$ é uma transformação natural. Como $\mathscr{F}$ e $\mathscr{G}$ são pré-feixes, temos que o morfismo induzido pela propriedade universal do kernel de $f(U)$, $\operatorname{Ker}(f(U)) \rightarrow \operatorname{Ker}(f(U))$ é a identidade de $\operatorname{Ker}(f(U))$.

Agora sejam $U \subseteq V \subseteq W$ abertos de $X$, a condição de pré-feixe nos garante que cada 
um dos quadrados e o retângulo maior do diagrama abaixo comutam:

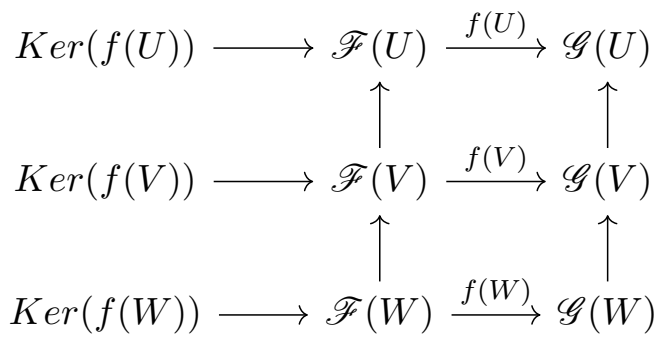

Pela comutatividade do diagrama e por propriedade do kernel, existem morfismo únicos $\operatorname{Ker}(f(W)) \rightarrow \operatorname{Ker}(f(V))$ e $\operatorname{Ker}(f(V)) \rightarrow \operatorname{Ker}(f(U))$ além de $\operatorname{Ker}(f(W)) \rightarrow \operatorname{Ker}(f(U))$, de forma que

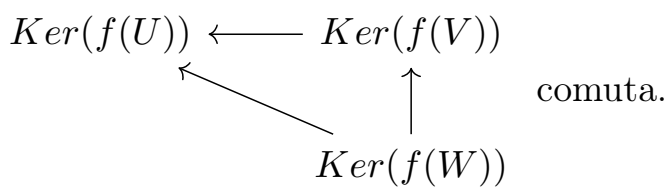

Procedemos de forma análoga para o cokernel.

Agora estamos preparados para fazermos as considerações necessárias sobre feixes.

Proposição 3.1.6 Sejam $\mathscr{F}, \mathscr{G} \in S h_{A b}(X)$, então $\mathscr{F} \oplus \mathscr{G} \in S h_{A b}(X)$.

\section{Demonstração:}

Seja $U$ aberto de $X$ e considere uma cobertura por $U_{i}, i \in I$. Seja $\left(s_{i}\right)_{i \in I}$ uma família compatível de $(\mathscr{F} \oplus \mathscr{G})\left(U_{i}\right)$. Assim $s_{i}=f_{i}+g_{i}$ para únicas $f_{i} \in \mathscr{F}\left(U_{i}\right)$ e $g_{i} \in \mathscr{G}\left(U_{i}\right)$. Pela unicidade da decomposição em $\mathscr{F}\left(U_{i}\right) \oplus \mathscr{G}\left(U_{i}\right)$, temos que $f_{i} \in \mathscr{F}\left(U_{i}\right)$ e $g_{i} \in \mathscr{G}\left(U_{i}\right)$ são famílias compatíveis para todo $i \in I$. Então:

$$
\begin{aligned}
f_{i_{U_{i} \cap U_{j}}}+g_{i_{U_{i} \cap U_{j}}}=\left(f_{i}+g_{i}\right)_{\left.\right|_{U_{i} \cap U_{j}}} & =\left.s_{i}\right|_{U_{i} \cap U_{j}} \\
& =s_{j_{U_{i} \cap U_{j}}} \\
& =\left(f_{j}+g_{j}\right)_{\left.\right|_{U_{i} \cap U_{j}}} \\
& =f_{j_{U_{i} \cap U_{j}}}+g_{j_{U_{i} \cap U_{j}}}
\end{aligned}
$$

Logo, como $\mathscr{F}$ e $\mathscr{G}$ são feixes, existem únicas almagamações $f \in \mathscr{F}(U)$ e $g \in \mathscr{G}(U)$.

Fazemos $s=f+g \in \mathscr{F}(U) \oplus \mathscr{G}(U)$, então

$$
s_{\left.\right|_{U_{i}}}=(f+g)_{\left.\right|_{U_{i}}}=f_{\left.\right|_{U_{i}}}+g_{\left.\right|_{U_{i}}}=f_{i}+g_{i}=s_{i}
$$

Portanto, existe $s$ tal que $s_{\left.\right|_{i}}=s_{i}, \forall i \in I$.

Agora vamos mostrar a unicidade da colagem.

Faça $s=f+g$ e $s^{\prime}=f^{\prime}+g^{\prime}, \operatorname{com} f, f^{\prime} \in \mathscr{F}(U)$ e $g, g^{\prime} \in \mathscr{G}(U)$. Então $s_{\left.\right|_{U_{i}}}=s_{\left.\right|_{U_{i}}}^{\prime}$ implica $f_{\left.\right|_{U_{i}}}+g_{\left.\right|_{U_{i}}}=f_{\left.\right|_{U_{i}}}^{\prime}+g_{\left.\right|_{U_{i}}}^{\prime}$, logo, $f_{\left.\right|_{U_{i}}}=f_{\left.\right|_{U_{i}}}^{\prime}$ e $g_{\left.\right|_{U_{i}}}=g_{\left.\right|_{U_{i}}}^{\prime}$ Como $\mathscr{F}$ e $\mathscr{G}$ são feixes, temos $f=f^{\prime}$ e $g=g^{\prime}$. Então $s=f+g=f^{\prime}+g^{\prime}=s^{\prime}$, como queríamos.

Corolário 3.1.7 A categoria $S h_{A b}(X)$ é aditiva.

\section{Demonstração:}

O Lema 3.1.6 nos garante que $S h_{A b}(X)$ possui biproduto e o feixe terminal será o mesmo terminal dos pré-feixes, verificar que o funtor constante também é feixe consiste de uma manipulação similar aos casos anteriores. Assim $S h_{A b}(X)$ é uma categoria aditiva.

Para mostrar que a categorias dos feixes de grupos abelianos é abeliana vamos precisar relembrar do que fizemos na seção 2.1 quando pouco a pouco transformamos um pré-feixe 
em um feixe. Na ocasião estávamos trabalhando com feixes de conjuntos, mas o caso dos feixes algébricos não é muito diferente.

Recorde que logo após a Definição 2.1.11 vimos que os stalks de $\mathscr{F}$ (aqui $\mathscr{F}$ é apenas um pré-feixe) em $x \in X$ são $\mathscr{F}_{x}=\underset{x \in U}{\lim _{x}} \mathscr{F}(U)$.

Se tivermos um morfismo entre pré-feixes $f: \mathscr{F} \rightarrow \mathscr{G}$, vamos denotar por $f_{x}: \mathscr{F}_{x} \rightarrow \mathscr{G}_{x}$ o morfismo entre grupos induzidos pelas $f$.

Vamos chamar de $\widetilde{\mathscr{F}}$ o feixe obtido através do pré-feixe $\mathscr{F}$ e de $i_{\mathscr{F}}: \mathscr{F} \rightarrow \widetilde{\mathscr{F}}$ o morfismo da feixificação.

Indicamos a seção sobre Localização de [Ive84] para ver em mais detalhes o que comentamos acima.

Teorema 3.1.8 A categoria $S h_{A b}(X)$ é abeliana.

\section{Demonstração:}

Definimos o Kernel e o Cokernel da mesma forma como da Proposição 3.1.5 de forma que resta apenas mostrar ambos são feixes.

Seja $U$ aberto de $X$ coberto por uma família de $U_{i}^{\prime}$ s, é rápido ver que $((\operatorname{Ker} f)(U))_{U \in \mathcal{C}^{o p}}$ é feixe.

$$
\begin{aligned}
& \operatorname{Ker}\left(f\left(U_{i}\right)\right) \stackrel{\gamma_{U_{i}}}{\longrightarrow} \mathscr{F}\left(U_{i}\right) \\
& \operatorname{Ker}(f(U)) \stackrel{\gamma_{U}}{\longrightarrow} \mathscr{F}(U)
\end{aligned}
$$

então $\gamma_{U}\left(s_{i}\right)_{U_{i}}=\gamma_{U}\left(s_{i}^{\prime}\right)_{U_{i}}$ implica $\gamma_{U}(s)=\gamma_{U}\left(s^{\prime}\right)$. Como $\mathscr{F}$ é feixe, $s=s^{\prime}$

Agora seja $s_{i} \in \operatorname{Kerf}\left(U_{i}\right)$ tal que $s_{i_{\left.\right|_{i} \cap U_{j}}}=s_{j_{U_{i} \cap U_{j}}}$. Usando $\gamma_{U_{i}}: \operatorname{Ker}\left(f\left(U_{i}\right) \rightarrow \mathscr{F}\left(U_{i}\right)\right.$ como acima, temos:

$$
s_{i} \in \operatorname{Kerf}\left(U_{i}\right) \Longrightarrow \gamma_{U_{i}} s_{i} \in \mathscr{F}\left(U_{i}\right) \Longrightarrow \exists t \in \mathscr{F}(U) \text { tal que } t_{\left.\right|_{i}}=\gamma_{U_{i}} s_{i}
$$

Pois $\mathscr{F}$ é feixe. Dessa forma:

$$
0=f \circ s_{i}=f \circ s_{\left.\right|_{i}}=(f \circ s)\left(U_{i}\right) \forall i \in I \Longrightarrow t \in \operatorname{Kerf}
$$

Teremos que feixificar o cokernel, mas temos que garantir que ainda é um cokernel. Considere o diagrama de cokernel estendido pelo morfismo $i_{\text {Cokerf }}:$ Cokerf $\rightarrow \widehat{\text { Cokerf }}$. Seja $\mathscr{H}$ um feixe de grupo abeliano e $h: \mathscr{G} \rightarrow \mathscr{H}$ tal que $h \circ f=0$. Assim, pela propriedade universal do cokernel existe um único $\bar{h}: \operatorname{Coker}(f) \rightarrow \mathscr{H}$ tal que pela propriedade da feixificação existe um único morfismo $\widetilde{\text { Coker } f} \rightarrow \mathscr{H}$.

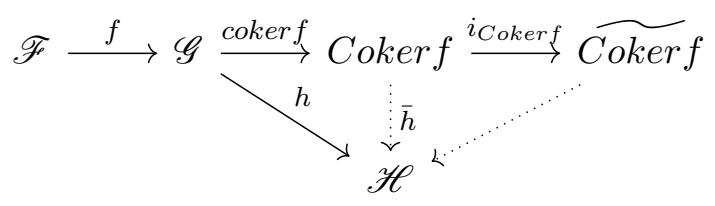

Isso nos garante que $\widetilde{\text { Coker } f}$ é de fato um cokernel.

Se provarmos que $\bar{f}: \operatorname{Coker}(k e r f) \rightarrow \operatorname{Ker}(\operatorname{cokerf})$ é iso, pela Proposição 1.1.21 concluímos a demonstração.

Acima vimos que a feixificação preserva cokerneis, como kerneis já são feixes, também preserva kerneis. Pelo Lema 2.1.16(iii), obtemos que nos stalks $\bar{f}_{x}:(\operatorname{Coker}(\text { ker } f))_{x} \rightarrow$ $(\operatorname{Ker}(\operatorname{cokerf}))_{x}$ é iso para todo $x$. Pelo Lema 2.1.16(ii) temos que $\bar{f}: \operatorname{Coker}(k e r f) \rightarrow$ $\operatorname{Ker}(\operatorname{cokerf})$ é iso. 


\subsection{Cohomologia de Feixes}

Antes de partirmos para a Cohomologia em feixes de casais $(\mathcal{C}, J)$, vamos definir grupos de cohomologia de feixes. Isso é feito através dos funtores derivados à direita do funtor das seções globais. É essencial portanto que demonstremos que dado um espaço topológico $X$ a categorias dos feixes $S h_{A b}(X)$ além de ser abeliana possui suficiente injetivos. Seguimos [Ive84] sendo mais detalhistas em demonstrações em que o livro não é mas deixando de lado alguns detalhes mais cuidadosamente trabalhados na referência, e explicitamos quando é o caso de ter mais atenção no decorrer do texto.

Teorema 3.2.1 A categoria $S h_{A b}(X)$ possui suficientes objetos injetivos.

\section{Demonstração:}

Vamos usar um fato bastante conhecido sobre grupos abelianos: um objeto $D \in A b$ é injetivo se, e somente se, $D$ é um grupo divisível, isto é, para todo $y \in D$ e para todo $n \in \mathbb{Z}$, existe $x \in D$ tal que $n x=y$.

Precisaremos de um morfismo geométrico chamado ponto $x$. Assim $x: \operatorname{Sets} \rightarrow \operatorname{Sh}(X)$. Dado um feixe $\mathscr{F}$, a imagem inversa $x^{*} \mathscr{F}$ é o stalk $\mathscr{F} x$ e a imagem direta é uma construção conhecida por "skyscraper sheaf". Dado $H$ conjunto e $U$ aberto em $X$ :

$$
\left(x_{*} H\right)(U)= \begin{cases}H, & x \in U \\ \{*\}, & \text { caso contrário }\end{cases}
$$

Onde $\{*\}$ é conjunto unitário.

Na versão abeliana temos $x^{*}: S h_{A b}(X) \rightarrow A b, \mathscr{F} \mapsto \mathscr{F}_{x}$ e $x_{*}: A b \rightarrow S h_{A b}(X)$, $H \mapsto\left(x^{*} H\right)(U)$. Para mais detalhes dessas afirmações veja VII.5 de [MLM94].

Para cada $x \in X$ escolhemos $D_{x}$ injetivo em $A b$ e um mono $\mathscr{F}_{x} \rightarrow D_{x}$.

Defina um feixe $D=\prod_{x \in X} x_{*} D_{x} \in S h(X)$. Assim, pelo Lema 2.1.16(i), $\mathscr{F}_{x} \rightarrow D_{x}$ induz um monomorfismo $\mathscr{F} \rightarrow D$.

Agora observe que dados $\mathscr{G}, \mathscr{G}^{\prime}$ em $\operatorname{Sh}_{A b}(X)$ :

$$
\begin{aligned}
\prod_{x \in X} \operatorname{Hom}_{A b}\left(\mathscr{G}_{x}, D_{x}\right) \cong \prod_{x \in X} \operatorname{Hom}_{A b}\left(x^{*} \mathscr{G}, D_{x}\right) & \cong \prod_{x \in X} \operatorname{Hom}_{S h_{A b}(X)}\left(\mathscr{G}, x_{*} D_{x}\right) \\
& \cong \operatorname{Hom}_{S h_{A b}(X)}\left(\mathscr{G}, \prod_{x \in X} x_{*} D_{x}\right) \\
& \cong \operatorname{Hom}_{S h_{A b}(X)}(\mathscr{G}, D)
\end{aligned}
$$

Usando o Lema 2.1.16(i), a comutatividade do diagrama

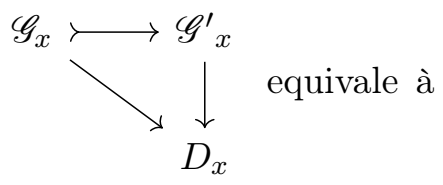

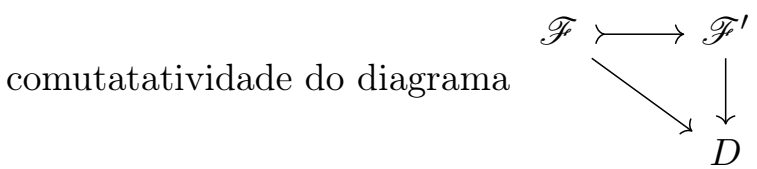

Ou seja, a injetividade de $D_{x}$ em $A b$ implica na injetividade de $D$ em $S h(X)$.

Definição 3.2.2 Dado um pré-feixe $\mathscr{F}$ em $X$ e um aberto $U$ de $X$, definimos as seções de $\mathscr{F}$ sobre $U$

$$
\Gamma(U, \mathscr{F})=\mathscr{F}(U)
$$

Os elementos $\Gamma(X, \mathscr{F})$ são ditos seções globais de $\mathscr{F}$

Dessa forma $\Gamma(X,-): S h_{A b}(X) \rightarrow A b$ é um funtor exato à esquerda. 
Definição 3.2.3 O q-ésimo grupos de cohomologia de $X$ com coeficientes em $\mathscr{F}$ é o $q$-ésimo funtor derivado de $\Gamma(X, \mathscr{F})$ e é denotado por $\mathrm{H}^{q}(X, \mathscr{F})$.

Em outras palavras, dada uma resolução injetiva $\mathscr{F} \rightarrow I^{\bullet}$ temos

$$
\mathrm{H}^{q}(X, \mathscr{F})=R^{q} \Gamma\left(X, I^{\bullet}\right)
$$

Definição 3.2.4 Um feixe $\mathscr{F}$ em $X$ é dito flácido se para qualquer aberto $U$ de $X$ temos que a restrição $\operatorname{res}_{U}^{X}: \mathscr{F}(X) \rightarrow \mathscr{F}(U)$ é sobrejetora.

Note que, equivalentemente, se temos um par de abertos $U \subseteq V, \mathscr{F}$ é flácido se $\mathscr{F}(V) \rightarrow$ $\mathscr{F}(U)$ é sobrejetora.

Notação: Seja $s$ uma seção global do feixe, isto é, $s \in \mathscr{F}(U)$ então usaremos $\operatorname{res}_{U}^{X}(s)=$ $s_{U}$.

Vamos mostrar alguns resultados sobre feixes flácidos.

Proposição 3.2.5 O feixe imagem direta de um feixe flácido é flácido.

\section{Demonstração:}

Seja $V$ aberto de $Y$, considere $f: Y \rightarrow X$ contínua. Suponha $\mathscr{F} \in S h(Y)$ o nosso feixe flácido, então, para qualquer aberto $V$ de $Y, \mathscr{F}(Y) \rightarrow \mathscr{F}(V)$ é sobrejetora.

Como $U \subseteq X$ implica $f^{-1}(U) \subseteq f^{-1}(X)$ e esses são dois abertos de $Y$, pois $f$ é contínua, obtemos que $\mathscr{F}\left(f^{-1}(X)\right) \rightarrow \mathscr{F}\left(f^{-1}(U)\right)$ é sobrejetora. Aplicando a definição de imagem direta, concluímos que $f_{*} \mathscr{F}(X) \rightarrow f_{*} \mathscr{F}(U)$ é sobrejetora, ou seja, $f_{*} \mathscr{F}$ é flácido.

Proposição 3.2.6 Produto de feixes flácidos é flácido.

\section{Demonstração:}

Seja $\left\{\mathscr{F}_{i}\right\}_{i \in I}$ uma família de feixes flácidos e $U$ aberto de $X$, então os morfismos $\mathscr{F}_{i}(X) \stackrel{f_{i}}{\longrightarrow} \mathscr{F}_{i}(U)$ são sobrejetores para todo $i=1, \ldots, n$.

Além disso, o morfismo entre o feixe de produtos

$$
\left(\prod_{i \in I} \mathscr{F}_{i}\right)(X) \rightarrow\left(\prod_{i \in I} \mathscr{F}_{i}\right)(U)
$$

é isomorfo ao morfismo do produto de feixes

$$
\prod_{i \in I}\left(\mathscr{F}_{i}(X)\right) \stackrel{\prod_{i \in I} f_{i}}{\longrightarrow} \prod_{i \in I}\left(\mathscr{F}_{i}(U)\right)
$$

Como produto de sobre é sobre, temos que $\prod_{i \in I} \mathscr{F}_{i}$ é feixe flácido.

Proposição 3.2.7 Todo feixe injetivo em $S h_{A b}(X)$ é flácido.

\section{Demonstração:}

Na Demonstração do Teorema 1.2.1 construímos um feixe $D=\prod_{x \in X} x_{*} D_{x}$, pela definição de $x_{*}: A b \rightarrow S h_{A b}(X)$ é imediato que $D(X) \rightarrow D(U)$ é sobrejetor.

Então, dado um feixe injetivo $\mathscr{I}$ sempre podemos obter um mono $i: \mathscr{I} \rightarrow D$, com $D$ feixe flácido.

Seja $f: D \rightarrow \mathscr{I}$ tal que $f \circ i=i d_{\mathscr{I}}$. Assim, como $i d_{\mathscr{I}}$ é iso, em particular é epi, $f$ é epi.

Dado $U$ aberto em $X$ também temos $f(U): D(U) \rightarrow \mathscr{I}(U)$ onde o seguinte diagrama comuta 


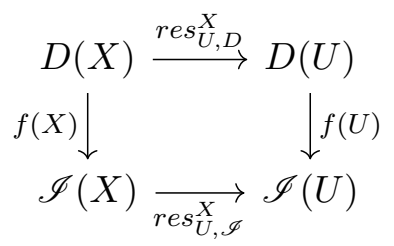

Como $f(U)$ e $r e s_{U, D}^{X}$ são sobrejetores, a composição $f(U) \circ r e s_{U, D}^{X}$ também é, logo, uma vez que o diograma comuta, temos $r e s_{U, \mathscr{D}}^{X}$ é sobrejetora e, portanto, $\mathscr{I}$ é flácido.

Proposição 3.2.8 Sejam $\mathscr{F}$ e $\mathscr{G}$ feixes flácidos e $0 \rightarrow \mathscr{F} \stackrel{f}{\rightarrow} \mathscr{G} \stackrel{g}{\rightarrow} \mathscr{H} \rightarrow 0$ uma sequência exata. Então $\mathscr{H}$ é flácido.

\section{Demonstração:}

Como o funtor das seções é exato à esquerda, temos imediatamente que a sequência

$$
0 \longrightarrow \Gamma(X, \mathscr{F}) \stackrel{\Gamma f}{\longrightarrow} \Gamma(X, \mathscr{G}) \stackrel{\Gamma g}{\longrightarrow} \Gamma(X, \mathscr{H})
$$

é exata. É possível verificar também que apenas por $\mathscr{F}$ ser flácido já se obtem que

$$
0 \longrightarrow \Gamma(X, \mathscr{F}) \stackrel{\Gamma f}{\longrightarrow} \Gamma(X, \mathscr{G}) \stackrel{\Gamma g}{\longrightarrow} \Gamma(X, \mathscr{H}) \longrightarrow 0
$$

é exata. Sem entrar em detalhes, o que se faz é definir uma família de pares $(U, t)$ de abertos $U$ de $X$ e seções $t \in \Gamma(U, \mathscr{G})$ tais que $(\Gamma g)(t)=s_{\left.\right|_{U}}$, onde $s \in \Gamma(X, \mathscr{H})$. Pelo Lema de Zorn temos um par maximal $(U, t)$, onde dizemos que $(U, t) \leq\left(U^{\prime}, t^{\prime}\right)$ se $U \subseteq U^{\prime}$ e $t_{\left.\right|_{U}}^{\prime}=t$. Supondo que $\Gamma g$ não é sobrejetora encontramos um aberto $U \cup V$ e uma extensão $t^{\prime}$ de $t$ que contraria a maximalidade de $(U, t)$.

A sequência é exata não apenas para $X$, mas para todo aberto $U$ de $X$, pois flácido em um aberto $U$ determina feixe flácido. Assim, obtemos o seguinte diagrama comutativo.

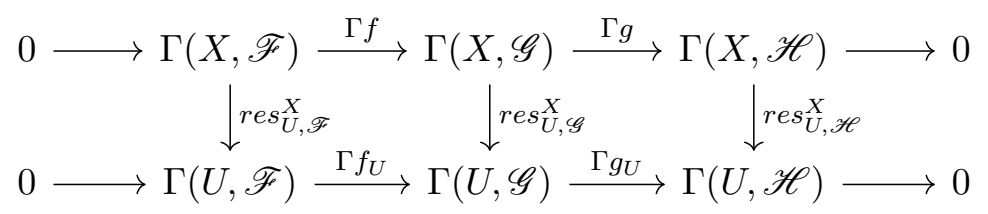

Como $\mathscr{G}$ é flácido, a composição $\Gamma g_{U} \circ \operatorname{res}_{U, \mathscr{G}}^{X}$ é sobrejetora e, pela comutatividade do diagrama, $\operatorname{res}_{U, \mathscr{H}}^{X}$ também é sobre, de onde segue que $\mathscr{H}$ é flácido.

Proposição 3.2.9 Seja $\mathscr{I}$ feixe em $X$. Se $\mathscr{I}$ é flácido, então $\Gamma(X,-)$-acíclico, isto é, $H^{q}(X, \mathscr{I})=0$ para todo $q>0$.

\section{Demonstração:}

Seja $\mathscr{I}$ feixe flácido em $S h_{A b}(X)$, temos uma sequêcia exata

$$
0 \longrightarrow \mathscr{I} \stackrel{f}{\longrightarrow} \mathscr{J} \stackrel{g}{\longrightarrow} \mathscr{H} \longrightarrow 0
$$

Como $S h_{A b}(X)$ possui suficientes injetivos, $\mathscr{J}$ é injetivo e, pela Proposição $3.2 .7, \mathscr{I}$ também é flácido.

Como comentamos em 3.2.8, $\mathscr{I}$ flácido nos dá uma sequência exata

$$
0 \longrightarrow \Gamma(X, \mathscr{I}) \stackrel{\Gamma f_{0}}{\longrightarrow} \Gamma(X, \mathscr{J}) \stackrel{\Gamma g_{0}}{\longrightarrow} \Gamma(X, \mathscr{H}) \longrightarrow 0
$$

Vamos analisar uma parte da sequência exata longa induzida pela cohomologia

$$
\Gamma(X, \mathscr{J}) \stackrel{\Gamma g_{0}}{\longrightarrow} \Gamma(X, \mathscr{H}) \stackrel{\delta_{0}}{\longrightarrow} H^{1}(X, \mathscr{I}) \stackrel{f_{1}}{\longrightarrow} H^{1}(X, \mathscr{J})=0
$$


onde $H^{1}(X, \mathscr{J})=0$ pois $\mathscr{J}$ é injetivo. Como essa sequência é exata, por um dos teorema de isomorfismo, $H^{1}(X, \mathscr{I}) \cong \Gamma(X, \mathscr{H}) / \operatorname{Kerg}_{0}$.

Mas $g_{0}$ é sobrejetora (pela sequência exata anterior) logo, $\operatorname{Ker} \delta=\operatorname{Im} g_{0} \cong \Gamma(X, \mathscr{H})$. Então, $H^{1}(X, \mathscr{I})=0$.

Concluiremos a prova usando indução. Suponha $H^{q}(X, \mathscr{I})=0$ para $q>0$. Considere agora o seguinte trecho da sequência longa exata induzida

$$
H^{q}(X, \mathscr{H}) \stackrel{\delta_{q}}{\longrightarrow} H^{q+1}(X, \mathscr{I}) \stackrel{f_{q+1}}{\longrightarrow} H^{q+1}(X, \mathscr{J}) \rightarrow \ldots
$$

Por ser injetivo, $H^{q}(X, \mathscr{J})=0$. Pela Proposição, 3.2.8 $\mathscr{H}$ é flácido, usando a hipótese de indução, também temos $H^{q+1}(X, \mathscr{H})=0$. Portanto, $H^{q+1}(X, \mathscr{I})=0$.

Então $H^{q}(X, \mathscr{I})=0$ para todo $q>0$, ou seja, é $\mathscr{I} \Gamma(X,-)$-acíclico.

\subsection{Cohomologia de $\operatorname{Ab}(\mathcal{E})$}

Visto um pouco da Cohomologia em $S h(X)$, nessa seção vamos definir os grupos de Cohomologia em uma generalizição: em um topos $\mathcal{E}=S h(\mathcal{C}, J)$. Seguimos o capítulo 8 de [Joh14].

Vamos mostrar algumas propriedades do funtor esquecimento $\mathbf{A b}(\mathcal{E}) \rightarrow \mathcal{E}$, mas antes vamos introduzir uma definição.

Definição 3.3.1 Um par de morfismos $A \underset{g}{\stackrel{f}{\longrightarrow}} B$ é dito reflexivo se existe $s: B \rightarrow A$ tal que $f \circ s=g \circ s=i d_{B}$. Ainda, um coequalizador reflexivo é o coequalizador de um par reflexivo.

Proposição 3.3.2 O funtor esquecimento $E: \boldsymbol{A b}(\mathcal{E}) \rightarrow \mathcal{E}$

(i) Cria coequalizadores reflexivos.

(ii) Cria limites finitos. ${ }^{1}$

(iii) Reflete isomorfismos.

\section{Demonstração:}

(i) Seja $A \underset{k}{\stackrel{h}{\longrightarrow}} B$ par reflexivo em $\mathbf{A b}(\mathcal{E})$, temos um correspondente par reflexivo em $\mathcal{E}$ que chamaremos de $A \underset{g}{\stackrel{f}{\longrightarrow}} B$.

Seja $q: B \longrightarrow C$, em $\mathcal{E}$, o coequalizador reflexivo de $f$ e $g$.

O Exercício 0.1 de [Joh14] nos diz que o produto de dois coequalizadores reflexivos é também um coequalizador. Então, $A^{2} \underset{g \times g}{\stackrel{f \times f}{\longrightarrow}} B^{2} \stackrel{q \times q}{\longrightarrow} C^{2}$ é um diagrama de coequalizador.

Por um argumento de indução podemos provar que $A^{m} \stackrel{f^{m}}{\underset{g^{m}}{\longrightarrow}} B^{m} \stackrel{q^{m}}{\longrightarrow} C^{2}$ é diagrama de coequalizador para todo $m \geq 0$.

Temos também que $A^{0} \longrightarrow B^{0} \longrightarrow C^{0}$ é um coequalizador, com $A^{0} \cong B^{0} \cong$ $C^{0} \cong 1$, o terminal.

\footnotetext{
${ }^{1}$ De fato cria todos os limites pequenos.
} 
Assim os morfismos $e_{A}: 1 \longrightarrow A, i_{A}: A \longrightarrow A$ e $m_{A}: A^{2} \longrightarrow A$ e os morfismos $e_{B}: 1 \longrightarrow B, i_{A}: B \longrightarrow B$ e $m_{B}: B^{2} \longrightarrow B$ induzem, respectivamente, $e_{C}: 1 \longrightarrow C, i_{C}: A \longrightarrow C$ e $m_{C}: C^{2} \longrightarrow C$ tal que $q$ é um homomorfismo. Como $q^{n}$ é coequalizador para todo $n \in\{0,1,2,3\}$, temos que $q^{n}$ é epimorfismo para esses mesmos $n$ 's, isso vale por causa do diagrama da associatividade. Isso implica que $C$ satisfaz qualquer operação satisfeita em $B$, ou seja, os diagramas de objeto de grupo abeliano são satisfeitos em $C$. Para finalizarmos basta equipar $C$ com a estrutura de grupo abelino e temos que $q: B \longrightarrow C$ é, em $\mathbf{A b}(\mathcal{E})$, coequalizador de $A \underset{k}{\stackrel{h}{\longrightarrow}} B$.

(ii) Mostrar que cria equalizadores é um processo muito similar ao que vimos acima de forma que vamos apenas esboçar a prova.

Sejam $f^{\prime}: X \rightarrow A$ e $g^{\prime}: X \rightarrow B$ morfismo em $\mathbf{A b}(\mathcal{E})$ com correspondentes morfismos $f: X \rightarrow A$ e $g: X \rightarrow B$ em $\mathcal{E}$.

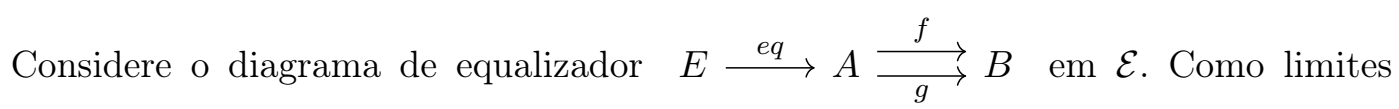
comutam com limites, o diagrama abaixo também é equalizador em $\mathcal{E}$ :

$$
E \times E \stackrel{e q \times e q}{\longrightarrow} A \times A \underset{g \times g}{\stackrel{f \times f}{\longrightarrow}} B \times B
$$

Logo, pela propriedade universal do equalizador, existe um único $m_{E}: E \times E \rightarrow E$ que faz o diagrama abaixo comutar

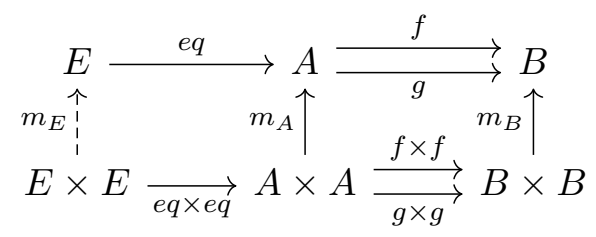

De onde seguem as leis diagramáticas de grupos abelianos.

Vamos mostrar que cria produtos finitos.

Sejam $f^{\prime}: X \rightarrow A$ e $g^{\prime}: X \rightarrow B$ morfismo em $\mathbf{A b}(\mathcal{E})$ com correspondentes morfismos $f: X \rightarrow A$ e $g: X \rightarrow B$ em $\mathcal{E}$. Considerere $u: X \rightarrow A \times B$ o único morfismo dado

pelo diagrama de produto

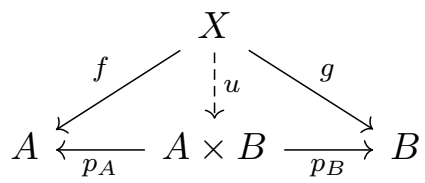

Por causa do isomorfismo $A \times B \cong B \times A$ conseguimos $m_{A \times B}:(A \times B) \times(A \times B) \rightarrow$ $A \times B$ induzido por $m_{A}: A \times A \rightarrow A$ e $m_{B}: B \times B \rightarrow B$ através do produto $m_{A} \times m_{B}$, essa expressão significa que o seguinte diagrama comuta:

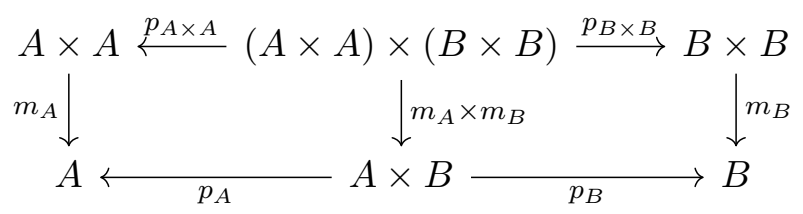

Ainda, fazemos $i_{A \times B}=i_{A} \times i_{B}$ e $e_{A \times B}=e_{A} \times e_{B}$, onde obtemos diagramas análogos ao acima.

Uma vez que $m_{A}$ e $m_{B}$ satisfazendo as condições de diagrama de objeto de grupo, o diagrama abaixo é comutativo 


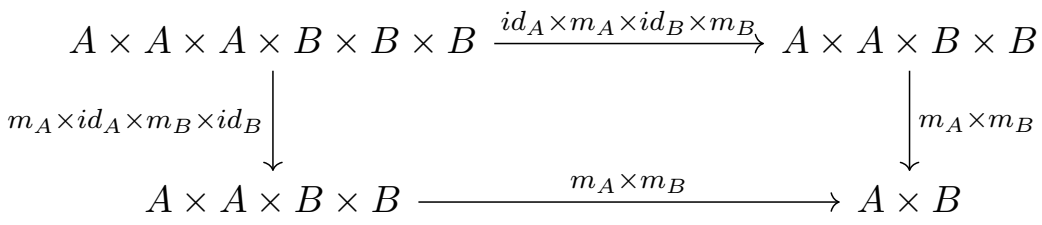

Obtemos assim a comutatividade do diagrama abaixo, pois é isomorfo ao diagrama logo acima.

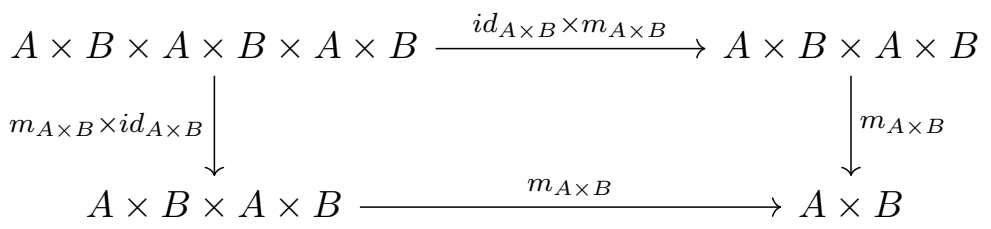

Isso significa que o produto $A \times B$ satisfaz o diagrama da lei da associatividade de objeto de grupo. Similarmente, usando $i_{A}, i_{B}, e_{A}$ e $e_{B}$, garantimos a comutatividade dos seguintes diagramas:
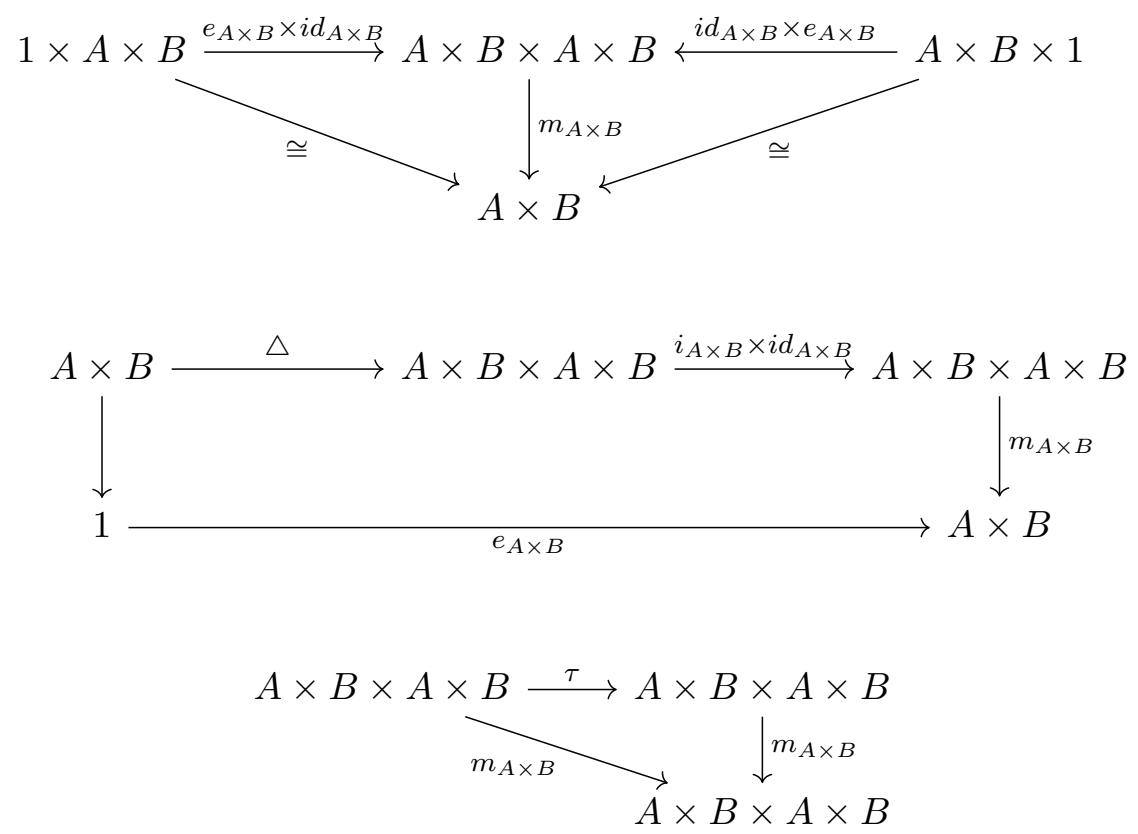

Então $A \times B$ pode ser visto como um objeto de grupo abeliano em $\mathcal{E}$ e o morfismo único $u: X \rightarrow A \times B$ em $\mathcal{E}$ induz um morfismo único em $\mathbf{A b}(\mathcal{E})$ que comuta com $m_{A \times B}, e_{A \times B}$ e $i_{A \times B}$.

(iii) É imediato, pois as condições envolvem a definição de objeto grupos abeliano em $\mathcal{E}$ são dadas apenas por diagramas comutativos.

Apesar de ser de amplo conhecimento, não encontramos na literatura nenhuma demonstração de que funtor esquecimento cria limites, mas mostramos aqui pois esse fato facilita muito a demontração de que $\mathbf{A b}(\mathcal{E})$ é uma categoria abeliana. A parte de criar coequalizadores reflexivos também é importante e está feita em [Joh14] (Lema 6.42) para $T(\mathcal{E})$, onde $T$ é uma teoria algébrica finitária, em vez de $\mathbf{A b}(\mathcal{E})$, no entanto os passos da demonstração são essencialmente os mesmos (afinal, podemos ver $A b$ como uma teoria algébrica finitária).

Estamos querendo definir os objetos de cohomologia de $\mathcal{E}$, devemos então verificar se $\mathbf{A b}(\mathcal{E})$ é realmente uma boa categoria para fazer isso. 
Fato 3.3.3 O funtor de esquecimento $E: \mathbf{A b}(\mathcal{E}) \rightarrow \mathcal{E}$ possui adjunto à esquerda. ${ }^{2}$

Teorema 3.3.4 Seja $\mathcal{E}$ um topos elementar, então $\boldsymbol{A b}(\mathcal{E})$ é uma categoria abeliana.

\section{Demonstração:}

Omitiremos a verificação de que a categoria $\operatorname{Ab}(\mathcal{E})$ é pré-aditiva pois os argumentos realmente interessantes encontram-se em ver que é abeliana. Vimos que o funtor esquecimento cria limites, logo, como $\mathcal{E}$ tem limites finitos, $\mathbf{A b}(\mathcal{E})$ possui limites finitos, para verificar que é uma categoria aditiva é necessário ver que o objeto inicial coincide com o terminal e que o produto coincide com o coproduto.

Suponha por um momento que $\mathcal{E}=S e t s$, então $\mathbf{A b}(\mathcal{E}) \simeq A b$. Seja 1 o objeto terminal de $A b$ (o grupo trivial), então, para todo $A \in A b$ existe um único morfismo $f: A \rightarrow 1$, mas também existe um único morfismo $g: 1 \rightarrow A$ para todo $A \in A b$, ou seja, 1 é também inicial e, portanto, nulo. Note que não foi utilizado qualquer tipo de argumento que desrepeitaria a lógica do topos. Da mesma forma, sabendo que produtos e coprodutos finitos em $A b$ coincidem, temos um biproduto em $\mathbf{A b}($ Sets $)$ que pode ser descrito apenas com argumentos válidos na lógica dos topos. Isso significa que esses argumentos podem ser descritos através de fórmulas e, de forma análoga ao Fato 2.6.5, garantimos a existência de objeto nulo e biproduto em um topos $\mathcal{E}$ qualquer. Além disso, se $X$ é um objeto de $\mathcal{E}$ munido de morfismos $m_{X}: X \times X \rightarrow X, i_{X}: X \times X$ e $e_{X}: 1 \times X$ onde valem as fórmulas das leis de grupo abelianos, isto é,

$$
\begin{gathered}
\forall x: X, y: X, z: X\left(m_{X}\left(m_{X}(x, y), z\right)=m_{X}\left(x,\left(m_{X}(y, z)\right)\right.\right. \\
\left.\forall x: X, 0: 1\left(m_{X}\left(e_{X} \times i d_{X}\right)(0, x)\right)=m_{X}\left(i d_{X} \times e_{X}(x, 0)\right)\right) \\
\forall x: X\left(m_{X}\left(i_{X} \times i d_{X}(\triangle(x))\right)=e_{X}(!(x))\right) \\
\forall x: X, y: X\left(m_{X}(x, y)=m_{X}(y, x)\right)
\end{gathered}
$$

Com isso queremos dizer que esse objeto $X$ de $\mathcal{E}$ equivale a um objeto de $\mathbf{A b}(\mathcal{E})$, onde essas leis são descritas de forma diagramática. Assim, a existênca de biproduto e objeto termnial em $\mathcal{E}$ implica na existências dos mesmos em $\mathbf{A b}(\mathcal{E})$, de onde segue que $\mathbf{A b}(\mathcal{E})$ é uma categoria aditiva.

Agora vamos provar que é abeliana. Começamos construindo o cokernel de $f: A \rightarrow B$ em $\operatorname{Ab}(\mathcal{E})$.

Seja $m: B \times B \rightarrow B$ o morfismo "multiplicação" de objeto de grupo de 2.6 .6 e $p_{B}$ : $A \times B \rightarrow B$ a projeção na segunda coordenada. Considere o coequalizador de $m \circ\left(f \times i d_{B}\right)$ e $p_{B}$.

Note que esse par $A \times B \stackrel{\text { 品 }\left(f \times i d_{B}\right)}{\longrightarrow} B$ é reflexivo, isto é, existe $s: B \rightarrow A \times B$ tal que $p_{B} \circ s=m \circ\left(f \times i d_{B}\right) \circ s=i d_{B}$. No caso, $s=\left(0_{B A}, i d_{B}\right)$.

Usando que $(A \times B) \times(A \times B) \longrightarrow B \stackrel{q \times q}{\longrightarrow} C$ é um diagrama de coequalizador (pelo Exercício 0.1 de [Joh14] produto de coequalizadores reflexivos é coequalizador) e obervando o diagrama abaixo, é possível mostrar que o coequalizador $q$ de $m \circ\left(f \times i d_{B}\right)$ e $p_{B}$ é o cokernel de $f$.

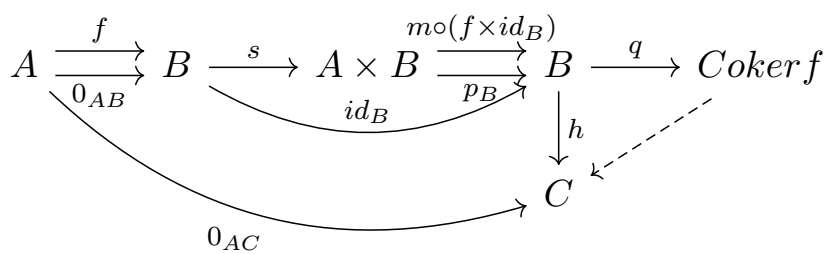

Veja que $f=i d_{B} \circ f=p_{B} \circ s \circ f=m \circ\left(f \times i d_{B}\right) \circ s \circ f$ e $0_{A B}=i d_{B} \circ 0_{A B}=$ $p_{B} \circ s \circ 0_{A B}=m \circ\left(f \times i d_{B}\right) \circ s \circ 0_{A B}$

\footnotetext{
${ }^{2}$ Ainda, como consequência dos itens $(i)$ e (iii) da Proposição 3.3.2, esse funtor é monádico - Teorema 0.13 de [Joh14].
} 
Agora seja $f$ de $\mathbf{A b}(\mathcal{E})$ um mono. Ao construir o cokernel obtivemos coker $f \circ f=0$, o que faremos é mostrar que $f$ é kernel de coker $f$, isto é, que para qualquer $f^{\prime}: A^{\prime} \rightarrow B$ tal que coker $f \circ f^{\prime}=0$ existe um único $u: A^{\prime} \rightarrow A$ onde $f^{\prime}=f \circ u$.

Sendo $f^{\prime}$ um morfismo de $\mathbf{A b}(\mathcal{E}), f^{\prime}$ é também um morfismo de $\mathcal{E}$. Pelo Teorema 2.4.14 garantimos que $f^{\prime}=m$ o $e$ para $m$ mono e $e$ um epi.

Se $f=m$ fazemos $u=e$, temos $f^{\prime}=f \circ u$ e para qualquer outro $u^{\prime}$ tal que $f \circ u=f \circ u^{\prime}$ temos que $u=u^{\prime}$, pois $f$ é mono e assim $u$ é único.

Se $f$ é outro mono que fatora $f^{\prime}$ então temos $f^{\prime}=f \circ u$, e $f=m \circ e^{\prime}$, de toda forma, pelo mesmo argumento, $u$ é único.

Assim encerramos que $f$ é kernel de seu próprio cokernel.

Agora seja $f$ de $\operatorname{Ab}(\mathcal{E})$ um epi, nesse caso, não apenas $f$ é um morfismo como é também epi em $\mathcal{E}$. Pelo Corolário 2.4.15, $f$ é um coequalizador, de onde construimos o cokernel similarmente ao que fizemos antes.

Observe que esse resultado nos faz obter trivialmente que a categoria dos feixes algébricos $S h_{A b}(X)$ é abeliana, pois $S h_{A b}(X) \simeq \mathbf{A b}(\mathcal{E})$ onde $\mathcal{E}$ é a categoria dos feixes de conjuntos $S h(X)$.

É possível mostrar que $\mathbf{A b}(\mathcal{E})$ é abeliana sem recorrer a argumentos de lógica interna e as propriedades do funtor esquecimento, para isso cada um dos diagramas devem ser exibidos explicitamente e, como podem imaginar, alguns são complicados, contudo, isso foi feito detalhadamente no artigo [Şte81].

Para a proposição a seguir precisaremos de resultados auxiliares, aos quais deixaremos apenas as referências das demonstrações:

Lema 3.3.5 1. Como $\boldsymbol{A b}(\mathcal{E}) \rightarrow \mathcal{E}$ cria coequalizadores reflexivos e $\mathcal{E}$ possui todos os coprodutos pequenos, então $\boldsymbol{A b}(\mathcal{E})$ possui todos os colimites pequenos.

2. Em $\mathcal{E}$, colimites filtrantes comutam com limites finitos.

\section{Demonstração:}

1. Feito para um caso mais geral em [Lin69] para casos mais gerais (Corolário 2, página $81)$.

2. Teorema 2.58 de [Joh14].

Proposição 3.3.6 Seja $\mathcal{E}$ um topos de Grothendieck, então $\boldsymbol{A b}(\mathcal{E})$ satisfaz o axioma AB5 de Grothendieck e possui gerador.

\section{Demonstração:}

Para mostrar que satisfaz AB5 devemos mostrar que $\mathbf{A b}(\mathcal{E})$ possui todos os colimites pequenos e todos os colimites pequenos filtrantes são universais.

Já vimos que o funtor esquecimento $\mathbf{A b}(\mathcal{E}) \rightarrow \mathcal{E}$ cria coequalizadores reflexivos (Proposição 3.3.1(i))e que $\mathcal{E}$ possui todos os coprodutos (Observação 2.3.7), disso obtemos que $\operatorname{Ab}(\mathcal{E})$ possui todos os colimites pequenos. Por 3.3.1(iii) temos ainda que o funtor esquecimento cria limites e, pelo Lema 3.3.5(ii), limites finitos comutam com colimites filtrantes. Dessa forma, os colimites filtrantes também são criados por $U$.

Novamente usando que $U$ cria limites, em particular, cria pullbacks e, pelo Corolário 2.4.13, todos os colimites são preservados por pullbacks e, portanto, temos que os colimites pequenos filtrantes em $\mathbf{A b}(\mathcal{E})$ são universais.

Por fim, pelo Teorema de Giraud, sabemos que $\mathcal{E}$ possui conjunto de geradores. Seja $\left\{G_{i}\right\}_{i \in I}$ esse conjunto. Veremos que o grupo abeliano livre $Z\left(\coprod_{i \in I} G_{i}\right)$, com $Z: \mathcal{E} \rightarrow$ $\mathbf{A b}(\mathcal{E})$, é um gerador de $\mathbf{A b}(\mathcal{E})$, sendo que esse funtor livre existe pelo Fato 3.3 .3 ( $Z$ é o adjunto à esquerda do funtor esquecimento). 
Sejam $f, g: X \rightarrow Y$ morfismos em $\mathbf{A b}(\mathcal{E})$, então $f$ e $g$ são morfismos em $\mathcal{E}$. Se $f \neq g$, como $\left\{G_{i}\right\}_{i \in I}$ é gerador de $\mathcal{E}$, existe $i \in I$ e $h_{i}: G_{i} \rightarrow E(X)$ tal que $E(f) \circ h_{i} \neq E(g) \circ g_{i}$. Considere $h: \coprod_{i \in I} G_{i} \rightarrow E(X)$ o morfismo universal do coproduto. Seja $\alpha_{1}$ a inclusão canônica $\alpha_{1}: G_{i} \rightarrow \coprod_{i \in I} G_{i}$, então $h_{i}=h \circ i_{1}$. Assim

$$
E(g) \circ h \circ \alpha_{1}=E(g) \circ h_{i} \neq E(f) \circ h_{i}=E(f) \circ h \circ \alpha_{1}
$$

Isso implica $E(g) \circ h \neq E(f) \circ h$.

Pela propriedade universal do coproduto, tome $k=\widetilde{h}: Z\left(\coprod_{i \in I} G_{i}\right) \rightarrow X$ em $\mathbf{A b}(\mathcal{E})$. Então, pela adjunção, $f \circ k=f \circ \widetilde{h} \neq g \circ \widetilde{h}=g \circ k$.

Portanto, $Z\left(\coprod_{i \in I} G_{i}\right)$ é gerador de $\mathbf{A b}(\mathcal{E})$.

Isso nos dá que $\mathbf{A b}(\mathcal{E})$ é uma categoria de Grothendieck quando $\mathcal{E}$ é um topos de Grothendieck.

O Teorema 1.10.1 de [Gro57] unido com a Proposição acima nos garantirá que $\mathbf{A b}(\mathcal{E})$ possui suficientes injetivos, segue seu enunciado abaixo.

Teorema 3.3.7 (Teorema de Grothendieck) Se $\mathcal{E}$ satisfaz o axioma AB5 e admite gerador, então qualquer objeto $A$ de $\mathcal{E}$ admite algum mono $m: A \rightarrow I$, onde $I$ é injetivo.

Teorema 3.3.8 Seja $\mathcal{E}$ topos de Grothendieck, então $\boldsymbol{A b}(\mathcal{E})$ possui suficientes injetivos.

\section{Demonstração:}

A Proposição 3.3.6 garante que $\mathbf{A b}(\mathcal{E})$ está nas hipóteses do Teorema de Grothendieck, assim obtemos imediatamente que possui suficientes injetivos.

No entanto, $\operatorname{Ab}(\mathcal{E})$ não possui suficientes projetivos. Desta forma não conseguimos construir os funtores derivados à esquerda que seriam essenciais para definir o grupos de homologia em um topos.

O teorema acima mostra que estamos de fato trabalhando com uma boa categoria para o nosso propóstito. Onde "boa" significa possuir suficientes injetivos, como vimos no capítulo de Álgebra Homológica, esta é uma condição necessária para construirmos funtores derivados à direta.

Contudo, precisamos ainda de mais um dado: precisamos de um funtor exato à esquerda.

Estamos lidando com topos de Grothendieck, isto é, $\mathcal{E}=S h(\mathcal{C}, J)$, para algum sítio $(\mathcal{C}, J)$. Ao tomarmos $A$ em $\mathbf{A b}(\mathcal{E}), A$ é um feixe em $\mathcal{C}, A(C)$ tem estrutura de grupo abeliano e os morfismos $C \rightarrow D$ em $\mathcal{C}$ induzem homomorfismos de grupos da forma $A(C) \rightarrow$ $A(D)$. Se considerarmos o o funtor das seções globais $\Gamma: \mathcal{E} \rightarrow$ Sets, que envia objeto de grupo abeliano em grupo abeliano, obtemos um funtor induzido $\Gamma_{\mathbf{A b}}: \mathbf{A b}(\mathcal{E}) \rightarrow \mathbf{A b}($ Sets $)$, dado por $\operatorname{Hom}_{\mathcal{E}}(1,-)$, que é imagem direta de $\Gamma$. Assim, $\Gamma_{\mathbf{A b}}$ é exato à esquerda e preserva injetivos, pois $\Gamma: \mathcal{E} \rightarrow$ Sets é um morfismo geométrico e vale o seguinte resultado:

Proposição 3.3.9 Seja $\mathcal{F} \rightarrow \mathcal{E}$ um morfismo geométrico. Então $f_{*}: \boldsymbol{A b}(\mathcal{F}) \rightarrow \boldsymbol{A b}(\mathcal{E})$ preserva injetivos.

\section{Demonstração:}

A ideia da demonstração é pegar um objeto injetivo de um lado e concluir algo no outro através de transposições que o morfimo geométrico permite por causa da adjunção.

Seja $E$ objeto injetivo em $\mathbf{A b}(\mathcal{F})$. Considere o diagrama abaixo em $\mathbf{A b}(\mathcal{E})$

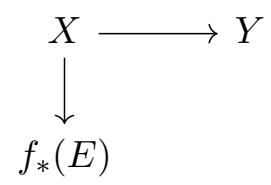

Transpondo tal diagrama: 


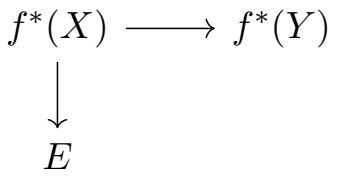

Como $E$ é injetivo podemos completar o diagrama acima e obter

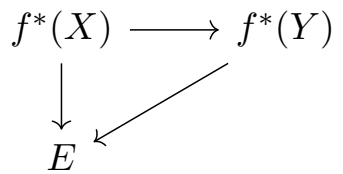

Por fim, transpomos o diagrama mais uma vez

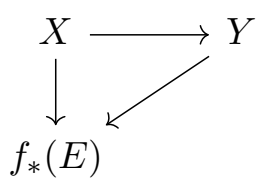

Ou seja, $f_{*}(E)$ é um objeto injetivo em $\mathbf{A b}(\mathcal{E})$

Com isso podemos definir sem medo a cohomologia de um topos de Grothendieck.

Definição 3.3.10 Seja $\mathcal{E}$ um topos de Grothendieck e $A$ um grupo abeliano em $\mathcal{E}$. O $q$-ésimo grupo de cohomologia de $\mathcal{E}$ com coeficientes em $A$ é o grupo

$$
H^{q}(\mathcal{E}, A)=R^{q}\left(\Gamma_{\mathbf{A b}}\right)(A)
$$

Em outras palavras, $H^{q}(\mathcal{E}, A)$ é q-ésimo funtor derivado à direita do complexo de cocadeias $\Gamma_{\mathbf{A b}} I^{0} \longrightarrow \Gamma_{\mathbf{A b}} I^{1} \longrightarrow \Gamma_{\mathbf{A b}} I^{2} \longrightarrow \ldots$ obtido de uma resolução injetiva

$$
0 \longrightarrow A \longrightarrow I^{0} \longrightarrow I^{1} \longrightarrow \ldots \text { em } \operatorname{Ab}(\mathcal{E})
$$

Podemos ainda definir o grupo de cohomologia para um certo objeto $X$ de $\mathcal{E}$ da seguinte forma: sabemos que $\operatorname{Hom}_{\mathcal{E}}(X,-)$ é exato à esquerda que manda um grupo abeliano $A \mathrm{em}$ um grupo de morfismos do tipo $X \rightarrow A \mathrm{em} \mathcal{E}$. Denotamos os derivados à direita desse funtor por $H^{q}(\mathcal{E}, X ; A)$. O funtor $X^{*}: \mathcal{E} \rightarrow \mathcal{E} \downarrow X$ que leva um objeto $Y$ no morfismo $Y \times X \rightarrow X$ induz um funtor $X_{A b}^{*}: A b(\mathcal{E}) \rightarrow A b(\mathcal{E} \downarrow X)$, o qual é exato e preserva injetivos. Assim conseguimos um isomorfismo $H^{q}(\mathcal{E}, X ; A) \cong H^{q}\left(\mathcal{E} \downarrow X, X_{A b}^{*}(A)\right)$ (ver página 262 de [Joh14]).

Para não sobrecarregar a notação iremos retirar o "índice" $A b$ dos funtores induzidos.

A partir de agora vamos usar outra poderosa ferramenta que introduzimos do capítulo de Álgebra Homológica, as sequências espectrais.

Teorema 3.3.11 Seja $f: \mathcal{F} \rightarrow \mathcal{E}$ um morfismo geométrico entre topos de Grothendieck.

(i) Se A é um grupo abeliano em $\mathcal{E}$, então existe um morfismo $H^{q}(\mathcal{E} ; A) \rightarrow H^{q}\left(\mathcal{F} ; f^{*} A\right)$, para todo $q \geq 0$.

(ii) Se $B$ é um grupo abeliano em $\mathcal{F}$, temos uma sequência espectral natural em $B$ $H^{p}\left(\mathcal{E} ; R^{q} f_{*}(B)\right) \Longrightarrow H^{p+q}(\mathcal{F} ; B)$. Essa é a conhecida sequência espectral de Leray.

\section{Demonstração:}


(i) Sejam $\Gamma: \mathcal{E} \rightarrow$ Sets e $\Lambda: \mathcal{F} \rightarrow$ Sets funtores seções globais. Vamos chamar de $\alpha: i d_{\mathcal{E}} \rightarrow f_{*} \circ f^{*}$ a unidade da adjunção $\left(f^{*} \dashv f_{*}\right)$. Por definição de funtor derivado à direita temos morfismos de conexão $R^{q}\left(\Gamma_{*}\right) \rightarrow R^{q+1}\left(\Gamma_{*}\right)$ que fornecem uma sequência exata longa.

Como $f^{*}$ preserva limites finitos, os funtores da forma $R^{q}\left(\Gamma_{*}\right) \circ f^{*}: \mathbf{A b}(\mathcal{E}) \rightarrow \mathbf{A b}$ também formam uma sequência longa exata, para todo $q \geq 0$.

Note que

$$
\Gamma_{*} f_{*} \cong \operatorname{Hom}_{\mathbf{A b}(\mathcal{F})}\left(1, f_{*}(-)\right) \cong \operatorname{Hom}_{\mathbf{A b}(\mathcal{E})}\left(f^{*} 1,(-)\right) \cong \operatorname{Hom}_{\mathbf{A b}(\mathcal{E})}(1,(-))=\Lambda_{*}
$$

Aplicando $\Gamma_{*}$ na unidade $\alpha$ obtemos $\Gamma_{*}(\alpha): \Gamma_{*} \rightarrow \Lambda_{*} \circ f^{*}$ transformação natural de obtemos morfismo induzido $R^{q}\left(\Gamma_{*}\right) \rightarrow R^{q}\left(\Lambda_{*}\right) \circ f^{*}$, por propriedade de funtor derivado. Aplicando $A \in \mathbf{A b}(\mathcal{E})$ :

$$
H^{q}(\mathcal{E}, A)=R^{q}\left(\Gamma_{*}\right) \rightarrow R^{q}\left(\Lambda_{*}\right) \circ f^{*}(A)=H^{q}\left(\mathcal{F}, f^{*} A\right)
$$

(ii) Sabemos que $\mathbf{A b}(\mathcal{E}), \mathbf{A b}(\mathcal{F})$ e $\mathbf{A b}(S e t s)$ são categorias abelianas e $f_{*}: \mathbf{A b}(\mathcal{F}) \rightarrow$ $\mathbf{A b}(\mathcal{E})$ e $\Gamma_{*}: \mathbf{A b}(\mathcal{E}) \rightarrow \mathbf{A b}($ Sets $)$ são funtores exatos à esquerda.

Basta agora aplicar o Teorema da Sequência Espectral de Grothendieck (Teorema 1.6.6) que obtemos uma sequência espectral $E_{r}^{p, q}$ de funtores $\mathbf{A b}(\mathcal{F}) \rightarrow \mathbf{A b}($ Sets $)$ tal que $E_{2}^{p, q}=\left(R^{p} \Gamma_{*}\right)\left(R^{q} f_{*}\right)$ e converge finitamente para $E_{\infty}^{p, q}$, que é filtração de $R^{p+q}\left(\Gamma_{*} f_{*}\right) \cong R^{p+q}\left(\Lambda_{*}\right)$.

Aplicando $B \in \mathbf{A b}(\mathcal{F})$, concluímos que $R^{p+q}\left(\Lambda_{*}\right)(B)=H^{p+q}(\mathcal{F} ; B)$

Proposição 3.3.12 Seja $f: \mathcal{F} \rightarrow \mathcal{E}$ um morfismo geométrico, com $\mathcal{E}=S h(\mathcal{C}, J)$. Então, para um grupo abeliano $A$ em $\mathcal{F}$ e q qualquer, $R^{q} f_{*}(A)$ é o $J$-feixe associado ao pré-feixe

$$
U \longmapsto H^{q}\left(\mathcal{F}, f^{*} l(U) ; A\right)
$$

em $\mathcal{C}$, onde $l: \mathcal{C} \rightarrow \operatorname{Sh}(\mathcal{C}, J)$ é o funtor canônico.

\section{Demonstração:}

Vamos começar supondo que $J$ é a topologia minimal, isto é, dado objeto $C$ de $\mathcal{C}$, $J(C)=\{$ o crivo maximal em $C\}$, dessa forma $\mathcal{E}=\operatorname{Sets}{ }^{\mathcal{C}^{o p}}$. Por definição de morfismo geométrico, $f^{*}$ preserva limites e é adjunto à esquerda de $f_{*}, \operatorname{logo}, f_{*}$ preserva limites pequenos e $f_{*}(-)(U)$ é exato à esquerda

Com isso obtemos que $R^{q} f_{*}(-)(U)$ é o q-ésimo funtor derivado à direita de $f_{*}(-)(U)$, pois $f_{*}(-)(U)$ é exato à esquerda, mas

$$
\begin{aligned}
f_{*}(-)(U) & \cong \operatorname{hom}_{\mathcal{E}}\left(h_{U}, f_{*}(-)\right) \\
& \cong \operatorname{hom}_{\mathcal{F}}\left(f^{*}\left(h_{U}\right),-\right) \\
& \cong H^{0}\left(\mathcal{F}, f^{*}\left(h_{U}\right),-\right): \mathbf{A} \mathbf{b}(\mathcal{F}) \rightarrow \mathbf{A b}(\text { Sets })
\end{aligned}
$$

Assim obtemos parcialmente o resultado desejado.

Para o caso em que $J$ é uma topologia de Grothendieck qualquer consideramos a inclusão $i: \mathcal{E} \rightarrow \operatorname{Sets}^{\mathcal{C}^{\text {op }}}$ e definimos um morfismo $g$ pela composição $g=i \circ f$.

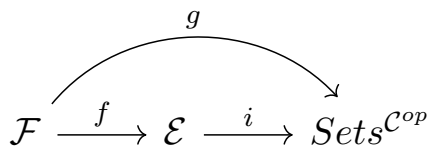


Desta forma $i^{*} g_{*}=\left(i^{*} i_{*}\right) f_{*} \cong f_{*}$. Porém, $i^{*}$ é exato e $i^{*} \cdot R^{q} g_{*} \cong R^{q}\left(i^{*} g_{*}\right) \cong R^{q}\left(f_{*}\right)$.

Além disso, $g^{*}\left(h_{U}\right)=f^{*} i^{*}\left(h_{U}\right)=f^{*} l(U)$, pois $i^{*}$ é o funtor feixe associado e $l$ é o funtor canônico.

Concluímos o resultado pelo que vimos acima para $\operatorname{Sets}^{\mathrm{C}^{o p}}$.

Como já dissemos, a existência de suficientes injetivos garante a existência de funtores derivados à direita e isso é o que nos dá a segurança para definir objetos de cohomologia, contudo, a via de resoluções injetivas não é a única forma de computar os grupos de cohomologia, às vezes, é mais conveniente considerar os grupos acíclicos de um determinado funtor. Para isso introduziremos uma definição e um lema útil.

Definição 3.3.13 Seja $A$ um grupo abeliano em um topos $\mathcal{E}$. Dizemos que $A$ é flácido se $H^{q}(\mathcal{E}, X ; A)=0$ para todo $q>0$ e para todo $X$ objeto de $\mathcal{E}$

Observe que essa denominação é uma certa simplificação. Uma vez que essa definição está completamente relacionada com o grupo de cohomologia, é um abuso de linguagem dizer que o grupo é flácido, no fundo estamos passando uma informação sobre as resoluções injetivas.

A Proposição 3.2 .9 parece ainda relacionar a noção de de feixe flácido acima com a definida em 3.2.4 para feixes de grupos abelianos sobre um espaço topológico. O resultado a seguir reforça isso:

Proposição 3.3.14 Seja $f: \mathcal{F} \rightarrow \mathcal{E}$ morfismo geométrico e $A$ um grupo abeliano flácido em $\mathcal{F}$. Então:

(i) A é acíclico para o funtor $f_{*}: \boldsymbol{A b}(\mathcal{F}) \rightarrow \boldsymbol{A} \boldsymbol{b}(\mathcal{E})$, isto é, $R^{q} f_{*}(A)=0, \forall q>0$.

(ii) $f_{*}(A)$ é flácido em $\mathcal{E}$

\section{Demonstração:}

(i) $A$ ser acíclico para $f_{*}$ significa $R^{q} f_{*}(A)=0, \forall q>0$. Isso ocorre pois $R^{q} f_{*}(A)$ é o $J$-feixe associado a $U \mapsto H^{q}\left(\mathcal{F}, f^{*} l(U) ; A\right)$, pela proposição anterior. Por definição de flácido, $H^{q}\left(\mathcal{F}, f^{*} l(U) ; A\right)=0, \forall q>0$.

(ii) Considerando a sequência espectral de Leray de $f$ temos:

$$
E_{2}^{p, q}=H^{p}\left(\mathcal{E}, R^{q} f_{*}(A)\right)= \begin{cases}H^{p}\left(\mathcal{E}, f_{*}(A)\right), & \text { se } q=0 \\ 0, & \text { se } q>0 \text { por }(\mathrm{i})\end{cases}
$$

Essa sequência converge para $E_{\infty}^{p, q}=R^{p+q}\left(f_{*}(A)\right)=H^{p+q}\left(\mathcal{E}, f_{*}(A)\right)$, que é uma filtração de $H^{p+q}(\mathcal{F} ; A)=0$, sempre que $p+q>0$.

Logo, $H^{p}\left(\mathcal{E}, f_{*}(A)\right)=0$, para todo $p>0$, ou seja, $f_{*}(A)$ é $H o m_{\mathcal{E}}(1,-)$-acíclico.

Note que $f_{*}(A)$ é $\operatorname{Hom}_{\mathcal{E}}(X,-)$-acíclico também, como veremos a seguir:

Da mesma forma como um objeto $X$ em $\mathcal{E}$ induz um funtor $X^{*}: \mathcal{E} \rightarrow \mathcal{E} \downarrow X$ (com respectivo adjunto $\left.X_{*}: \mathcal{E} \downarrow X \rightarrow \mathcal{E}\right)$, temos que o objeto $f^{*} X$ de $\mathcal{F}$ induz $\left(f^{*} X\right)^{*}: \mathcal{F} \rightarrow$ $\mathcal{F} \downarrow f^{*} X$ (com respectivo adjunto $\left(f^{*} X\right)_{*}: \mathcal{F} \downarrow f^{*} X \rightarrow \mathcal{F}$

Considere o morfismo geométrico $f \downarrow X: \mathcal{F} \downarrow f^{*} X \rightarrow \mathcal{E} \downarrow X$ de forma que comutatividade de um morfismo em $\mathcal{F} \downarrow f^{*} X$ é preservada em $\mathcal{E} \downarrow X$.

Desta forma, temos o seguinte diagrama comutativo:

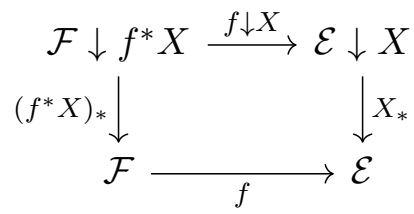


No Lema 2.5.8 introduzimos uma versão da Condição de Beck-Chevaley, aqui estamos diante de um caso um pouco diferente, mas basta a comutatividade do diagrama acima e considerar os funtores induzidos pela categoria de objetos de grupos abelianos que podemos obter um outro diagrama comutativo análogo ao do Lema 2.5.8 (veja a página 275 de [Joy08]). Então o seguinte diagrama comuta:

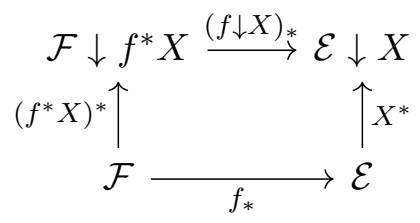

Assim temos um isomorfismo $X^{*} \circ f_{*}(A) \cong(f \downarrow X)_{*} \circ\left(f^{*} X\right)^{*}(A)$.

Vamos trabalhar com a sequência espectral de $(f \downarrow X)_{*} \circ\left(f^{*} X\right)^{*}$.

Então $E_{2}^{p, q}=\left(R^{p}(f \downarrow X)_{*}\right)\left(R^{q}\left(f^{*} X\right)^{*}\right)$, que converge para $E_{\infty}^{p, q}$, que é filtração de

$$
R^{p+q}\left((f \downarrow X)_{*} \circ\left(f^{*} X\right)^{*}\right) \cong R^{p+q}\left(X^{*} f_{*}(A)\right) \cong X^{*} R^{p+q} f_{*}(A) \cong 0, p+q>0
$$

$\operatorname{Mas} R^{p+q}\left(X^{*} f_{*}(A)\right)=H^{p+q}\left(\mathcal{E} \downarrow X ; X^{*} f_{*}(A)\right) \cong H^{p+q}\left(\mathcal{E}, X ; f_{*}(A)\right)$.

\subsection{Cohomologia de Čech}

Primeiro faremos a Cohomologia de Čech para feixes sobre espaços topológicos, sendo guiados por [Har77], depois veremos como proceder para topos de Grothendieck como feito em [Joh14].

Para a primeira parte dessa seção, fixemos $X$ um espaço topológico e $\mathscr{F}$ feixe de grupos abelianos sobre $X$. Dada uma cobertura $\bigcup_{i \in I} U_{i}=U$ de $X$ com $I$ conjunto de índices bem ordenado, para $i_{0}, \ldots, i_{q} \in I$ denotamos $U_{i_{0}, \ldots, i_{q}}=U_{i_{0}} \cap \ldots \cap U_{i_{q}}$. Assim:

Definição 3.4.1 O complexo de cocadeias de Čech é definido por

$$
C^{q}(U, \mathscr{F})=\prod_{i_{0}<\ldots<i_{p}} \mathscr{F}\left(U_{i_{0}, \ldots, i_{q}}\right), \forall q \geq 0
$$

Os morfismos de cobordo $d^{q}: C^{q}(U, \mathscr{F}) \rightarrow C^{q+1}(U, \mathscr{F})$ são dados por

$$
\left(d^{q} \alpha\right)=\left.\sum_{k=0}^{q+1}(-1)^{k} \alpha\left(\delta_{k}\right)\right|_{U_{i_{0}, \ldots, i_{q+1}}}
$$

onde $\alpha\left(\delta_{k}\right)=\alpha_{i_{0}, \ldots, \hat{i_{k}}, \ldots, i_{q+1}}$, isto é, $\delta_{k}$ indica que retiramos $i_{k}$.

Note que $\alpha_{i_{0}, \ldots, \widehat{i_{k}}, \ldots, i_{q+1}} \in \mathscr{F}\left(U_{i_{0}, \ldots, \hat{i_{k}}, \ldots, i_{q+1}}\right)$, por isso tomamos a restrição a $U_{i_{0}, \ldots, i_{q+1}}$ para garantir que caia em $C^{q+1}(U, \mathscr{F})$.

Observação 3.4.2 Veja que de fato temos um complexo de cocadeia, pois $d^{q+1} \circ d^{q}=0$.

Seja $\alpha \in C^{q}(U, \mathscr{F})$. Então:

$$
\begin{aligned}
\left(d^{q+1} \circ d^{q}\right)(\alpha) & =d^{q+1}\left(\sum_{k=0}^{q+1}(-1)^{k} \alpha\left(\delta_{k}\right)_{\left.\right|_{U_{0}, \ldots, i_{q+1}}}\right) \\
& =\sum_{l=0}^{q+2}(-1)^{l}\left(\sum_{k=0}^{q+1}(-1)^{k} \alpha\left(\delta_{k, l}\right)_{\left.\right|_{U_{i_{0}}, \ldots, i_{q+1}}}\right)_{\left.\right|_{U_{i_{0}}, \ldots, i_{q+2}}} \\
& =\sum_{l=0}^{q+2} \sum_{k=0}^{q+1}(-1)^{l+k} \alpha\left(\delta_{k, l}\right)_{\left.\right|_{U_{i_{0}}, \ldots, i_{q+2}}}
\end{aligned}
$$


onde $\alpha\left(\delta_{k, l}\right)$ indica que as $i_{k}$ e $i_{l}$ foram retirados. Cada termo da forma $U_{i_{0}, \ldots, \widehat{i_{l}}, \ldots, \widehat{i_{k}}, \ldots, i_{q+2}}$ aparece duas vezes mas com os sinais trocados quando $i_{l}$ e $i_{k}$ trocam de posição, assim, essa soma alternada se anula e $d^{q+1} \circ d^{q}=0$.

Sempre que tomamos um elemento $\alpha \in C^{q}(U, \mathscr{F})$ é conveniente usarmos $\alpha_{i_{0}, \ldots, i_{q}}$ para todas as $(q+1)$-uplas de elementos de $I$. Caso haja um repetição de índices em $\left\{i_{0}, \ldots, i_{q}\right\}$ convencionamos $\alpha_{i_{0}, \ldots, i_{q}}=0$ e se os índices forem todos distintos então $\alpha_{i_{0}, \ldots, i_{q}}=$ $(-1)^{\sigma} \alpha_{\sigma i_{0}, \ldots, \sigma i_{q}}$, onde $\sigma$ é a única permutação tal que $\sigma i_{0}<\ldots<\sigma i_{q}$.

Definição 3.4.3 Seja $\mathscr{F}$ um feixe de grupos abelianos em $X$, definimos $q$-ésimo grupo de cohomologia de Čech de $\mathscr{F}$ em relação a $U$ é o grupo

$$
\check{\mathrm{H}}^{q}(U, \mathscr{F})=\operatorname{Kerd}^{q} / \operatorname{Imd}^{q-1}, \forall q>0
$$

Os grupos de cohomologia de Čech não determinam uma teoria de Cohomologia, é apenas uma técnica para calcular a Cohomologia de feixes escolhendo uma cobertura de abertos do espaço topológico e a partir dele contruir um complexo, como fizemos acima. Uma vez que é uma ferramenta de cálculo de cohomologia, devemos providencias pelo menos um exemplo que iluestre a utilidade desse método.

Exemplo 3.4.4 Seja $X=S^{1}$ o círculo e $\mathscr{F}=\mathbb{Z}$ o feixe constante com fibra $\mathbb{Z}$, isto é, dado um aberto $U, \operatorname{Hom}(U, \mathbb{Z})=\mathscr{F}(U)$ é o conjunto das funções localmente constantes. Consideramos como cobertura $U$ dois semi-círculos abertos $U_{1}$ e $U_{2}$ que se sobrepõem nas pontas, de forma que a intersecção $U_{1} \cap U_{2}$ é formada por dois intervalos abertos.
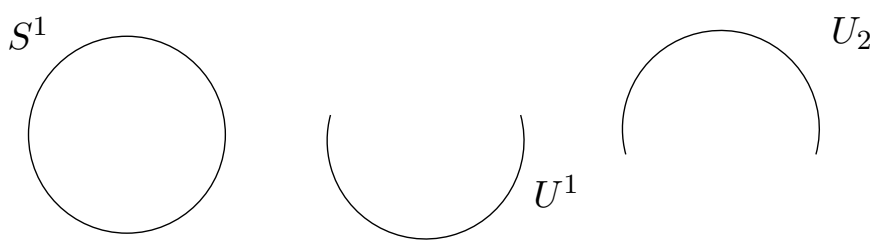

$$
U_{1} \cap U_{2}
$$

Os complexos de cocadeia são:

$$
C^{0}(U, \mathscr{F})=\mathscr{F}\left(U_{1}\right) \times \mathscr{F}\left(U_{2}\right)=\operatorname{Hom}\left(U_{1}, \mathbb{Z}\right) \times \operatorname{Hom}\left(U_{2}, \mathbb{Z}\right) \cong \mathbb{Z} \times \mathbb{Z}
$$

Pois $U_{1}$ e $U_{2}$ são abertos conexos.

$$
C^{1}(U, \mathscr{F})=\mathscr{F}\left(U_{1} \cap U_{2}\right)=H o m\left(U_{1} \cap U_{2}, \mathbb{Z}\right) \cong \mathbb{Z} \times \mathbb{Z}
$$

Pois $U_{1} \cap U_{2}$ é aberto com 2 componentes conexas.

Os morfismos de cobordo são:

$$
\begin{gathered}
d^{0}: C^{0}(U, \mathscr{F}) \rightarrow C^{1}(U, \mathscr{F}), d^{0}(<a, b>)=<b-a, b-a> \\
d^{1}: C^{1}(U, \mathscr{F}) \rightarrow 0, d^{1}(<a, b>)=0
\end{gathered}
$$

Logo, $K e r d^{0} \cong \mathbb{Z}, I m d^{0} \cong \mathbb{Z}, K e r d^{1} \cong \mathbb{Z} \times \mathbb{Z}$ e $I m d^{-1} \cong 0$, de onde segue:

$$
\check{\mathrm{H}}^{0}(U, \mathscr{F})=\mathbb{Z} \text { e } \check{\mathrm{H}}^{1}(U, \mathscr{F})=\mathbb{Z}
$$

Proposição 3.4.5 Seja $\mathscr{F}$ feixe de grupos abelianos em $X$, então $\check{\mathrm{H}}^{0}(U, \mathscr{F}) \cong \Gamma(X, \mathscr{F})$.

\section{Demonstração:}

Pela definição, $\check{\mathrm{H}}^{0}(U, \mathscr{F})=\operatorname{Ker}\left(d: C^{0}(U, \mathscr{F}) \rightarrow C^{1}(U, \mathscr{F})\right)$.

Seja $\alpha \in C^{0}(U, \mathscr{F})$ tal que $\alpha=\left(\alpha_{i} \in \mathcal{F}\left(U_{i}\right)\right)_{i \in I}$. Para cada par de índices $i<j$, temos $(d \alpha)_{i j}=\alpha_{j}-\alpha_{i}$. Então $\alpha$ pertence a Kerd se, e somente se, $(d \alpha)_{i j}=0$. 
Mas $(d \alpha)_{i j}=0$ se, e só se, $\alpha_{i}$ e $\alpha_{j}$ coincidem em $U_{i} \cap U_{j}$, isto é, $s_{\left.\right|_{U_{i} \cap U_{j}}}\left(\alpha_{i}\right)=s_{\left.\right|_{U_{i} \cap U_{j}}}\left(\alpha_{j}\right)$ e isso equivale a dizer que $\alpha$ pertence a $\Gamma(U, \mathscr{F})=\mathscr{F}(U)$.

Portanto, $\check{\mathrm{H}}^{0}(U, \mathscr{F}) \cong \Gamma(X, \mathscr{F})$.

Queremos estudar algumas situações em que a Cohomologia de Čech e a Cohomologia dos funtores derivados coincidem, para isso teremos coinsiderar uma versão feixificada de $C^{q}(U, \mathscr{F})$.

Definição 3.4.6 Seja $V$ aberto de $X$ e $f: V \rightarrow X$ o morfismo inclusão. Definimos a feixificação do complexo de Čech por

$$
\mathscr{C}^{q}(U, \mathscr{F})=\prod_{i_{0}<\ldots<i_{q}} f_{*}\left(\mathscr{F}_{U_{i_{0}, \ldots, i_{q}}}\right)
$$

Onde a restrição de feixe é dada por $\mathscr{F}_{\left.\right|_{i_{0}, \ldots, i_{q}}}(V)=\lim _{\overrightarrow{V C U}} \mathscr{F}(U)$.

Os morfismos de cobordo são o mesmos da Definição 3.4.1 e $f_{*}$ é a imagem direta tal como vimos no Exemplo 2.4.2

Com isso, para cada $q \geq 0, \Gamma\left(X, \mathscr{C}^{q}(U, \mathscr{F})\right)=C^{q}(U, \mathscr{F})$

Proposição 3.4.7 Seja $\mathscr{F}$ um feixe de grupos abelianos em $X$. O complexo $\mathscr{C} \bullet(U, \mathscr{F})$ é uma resolução de $\mathscr{F}$.

\section{Demonstração:}

Devemos exibir um morfismo $\mathscr{F} \rightarrow C^{0}(U, \mathscr{F})$ tal que a sequência

$$
0 \longrightarrow \mathscr{F} \longrightarrow C^{0}(U, \mathscr{F}) \longrightarrow C^{1}(U, \mathscr{F}) \longrightarrow \ldots
$$

é exata.

Recorde o que fizemos no Teorema 3.2.1, onde $x^{*} \mathcal{F}=\mathcal{F}_{x}$ Definimos $e: \mathscr{F} \rightarrow C^{0}(U, \mathscr{F})$ tomando o produto de $\mathscr{F} \rightarrow f_{*}\left(\mathscr{F}_{U_{i}}\right)_{i \in I}$. Desta forma é imediato dos axiomas de feixes que

$$
0 \longrightarrow \mathscr{F} \longrightarrow C^{0}(U, \mathscr{F})
$$

é exata. Devemos verificar a exatidão para valores $q>0$ e para isso basta provar a exatidão nos stalks (pois a coleção de funtores de stalk preservam e refletem kernel e cokernel).

Seja $x \in X$, suponha $x \in U_{j}$ para certo $i \in I$. Para cada $q \geq 1$ definimos um mapa $k$ : $\left(C^{q}(U, \mathscr{F})\right)_{x} \rightarrow\left(C^{q-1}(U, \mathscr{F})\right)_{x}$, com $(k \alpha)_{i_{0}, \ldots, i_{q-1}}=\alpha_{j, i_{0}, \ldots, i_{q-1}}$ onde $\alpha \in \Gamma\left(V, C^{q}(V, \mathscr{F})\right)$ representa $\alpha_{x} \in\left(C^{q}(U, \mathscr{F})\right)_{x}$ em que $V$ é vizinhaça de $x$ pequena o suficiente para $V \subseteq U_{j}$.

Uma vez que checarmos que $(d \circ k+k \circ d)(\alpha)=\alpha$ teremos que $k$ é uma homotopia de cadeia de $\left(C^{q}(U, \mathscr{F})\right)_{x}, q \geq 1$. Isso implica que o morfismo identidade e o morfismo nulo são homotópicas por cadeias, logo, pela Proposição $1.3 .10, H^{q}\left(C^{\bullet}(U, \mathscr{F})\right)=0$ para todo $q \geq 1$. Portanto, a sequência longa é exata como queríamos.

Checando que $(d \circ k+k \circ d)(\alpha)=\alpha$ :

Por um lado temos

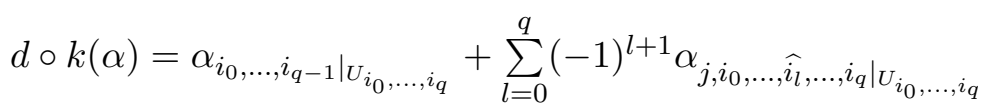

Por outro,

$$
k \circ d(\alpha)=k\left(\sum_{l=0}^{q+1}(-1)^{l} \alpha_{i_{0}, \ldots, \widehat{i_{l}}, \ldots,\left.i_{q}\right|_{U_{i_{0}}, \ldots, i_{q}+1}}\right)=\sum_{l=0}^{q}(-1)^{l} \alpha_{j, i_{0}, \ldots, \widehat{\hat{l}_{l}}, \ldots,\left.i_{q}\right|_{U_{i_{0}}, \ldots, i_{q}}}
$$

Desta forma as somas alternadas se anulam e $\alpha$, assim verificamos $(d \circ k+k \circ d)(\alpha)=\alpha$.

Vamos mostrar apenas mais dois resultados envolvendo a Cohomologia de Čech de feixes de grupos abelianos. 
Proposição 3.4.8 Seja $\mathscr{F}$ feixe de grupos abelianos flácido em $X$, então $\check{\mathrm{H}}^{q}(U, \mathscr{F})=$ $0, \forall q>0$

\section{Demonstração:}

Considere a resolução $0 \rightarrow \mathscr{F} \rightarrow C^{\bullet}(U, \mathscr{F})$. Como $\mathscr{F}$ é flácido e pela Proposição 3.2.6, o produto de flácidos $\prod_{i_{0}<\ldots<i_{q}} \mathscr{F}\left(U_{i_{0}, \ldots, i_{q}}\right)$ é flácido e, por 3.2.5, o feixe imagem direta $f_{*}\left(\mathscr{F}_{U_{i_{0}, \ldots, i_{q}}}\right)$ também é flácidao, então $C^{q}(U, \mathscr{F})$ é flácido.

Usando a Proposição 3.2 .9 e que $\Gamma\left(X, \mathscr{C}^{q}(U, \mathscr{F})\right)=C^{q}(U, \mathscr{F})$ temos $\check{\mathrm{H}}^{q}(U, \mathscr{F})=$ $H^{q}\left(\Gamma\left(X, \mathscr{C}^{q}(U, \mathscr{F})\right)=0\right.$ para todo $q>0$.

Proposição 3.4.9 Existe mapa $\check{\mathrm{H}}^{q}(U, \mathscr{F}) \rightarrow \mathrm{H}^{q}(X, \mathscr{F})$ natural e funtorial em $\mathscr{F}$ para cada $q \geq 0$

\section{Demonstração:}

Seja $0 \rightarrow \mathscr{F} \rightarrow \mathscr{I} \bullet$ resolução injetiva de $\mathscr{F}$ em $S h_{A b}(X)$. Considere uma outra resolução $0 \rightarrow \mathscr{F} \rightarrow C^{\bullet}(U, \mathscr{F})$. Pelo Teorema 1.4.6 existe um morfismo de complexos $f^{\bullet}: C^{\bullet}(U, \mathscr{F}) \rightarrow \mathscr{I} \bullet, \operatorname{logo}$, existe um morfismo $\Gamma\left(X, C^{\bullet}(U, \mathscr{F})\right) \rightarrow \Gamma(X, \mathscr{I})$.

Tomando o quociente do kernel pela imagem dos diferenciais desses complexos, obtemos o morfismo canônico $\check{\mathrm{H}}^{q}(U, \mathscr{F}) \rightarrow \mathrm{H}^{q}(X, \mathscr{F})$.

Com isso temos mais do que o suficiente de motivação para voltar nossa atenção para o caso dos topos de Grothendieck. Faremos o possível para evidenciar as semelhanças entre a Cohomologia de Čech dos feixes de grupos abelianos e do feixes de um site "abelianizados"pelos objetos de grupo.

Devemos esquecer a notação que fixamos no início da seção e fixar uma nova. De agora em diante manteremos $\mathcal{E}=S h(\mathcal{C}, J)$ topos de Grothendieck e $P=\operatorname{Sets}^{{ }^{\circ}{ }^{o p}}$ a correspondente categoria de pré-feixes. Sendo $i: \mathcal{E} \rightarrow P$ a inclusão canônica, denotaremos por $\mathcal{H}: \mathbf{A b}(\mathcal{C}) \rightarrow \mathbf{A b}(P)$ o $q$-ésimo funtor derivado à direita de $i_{*}$, isto é, $\mathcal{H}=R^{q} i_{*}$.

A proposição abaixo será necessária nos resultados seguintes:

Proposição 3.4.10 Seja $A \in \boldsymbol{A b}(\mathcal{E})$. Então, $\left(\mathcal{H}^{q}(A)\right)^{+}=0, \forall q>0$, onde $+: P \rightarrow P$ é o funtor de "semi-feixificação" introduzido na Definição 2.2.18

\section{Demonstração:}

Considere a composição $i^{*} \circ i_{*} \cong i d$. Lembre que a imagem inversa da inclusão é o funtor feixe associado $a$ que introduzimos na seção 2.2, conforme pontuamos no Exemplo 2.4.3.

$$
\left(\mathbf{A b}(\mathcal{E}) \stackrel{i_{*}}{\rightarrow} \mathbf{A b}(P) \stackrel{i^{*}}{\rightarrow} \mathbf{A b}(\mathcal{E})\right) \cong(\mathbf{A b}(\mathcal{E}) \stackrel{i d}{\rightarrow} \mathbf{A b}(\mathcal{E}))
$$

Pelo Teorema 1.6.6, existe uma sequência espectral $E_{r}^{p, q}=R^{p+q}\left(i^{*} \circ i_{*}\right)$ de $i^{*} \circ i_{*}$.

Uma vez que $i^{*}$ preserva limites, então preserva somas diretas e kerneis, isto é, é exato à esquerda. Logo, $R^{p}\left(i^{*}\right)=0$, de onde concluímos que $E_{2}^{p, q}=0$ para todo $p>0$. Além disso, como $i d_{\mathbf{A b}(\mathcal{E})}$ também é exato, $E_{\infty}^{p, q}=0$ para $p+q>0$.

Agora fixe $p=0$ e varie $q>0$. Assim temos uma sequência de diferenciais da forma
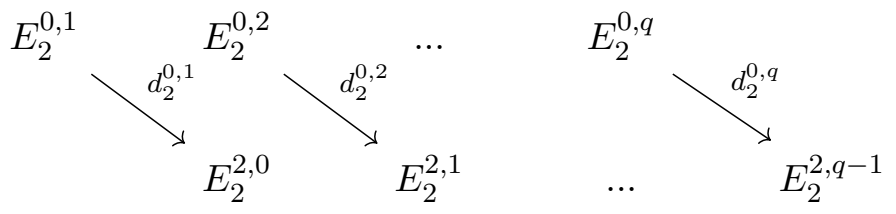

Como $E_{2}^{p, q}=0$ para todo $p>0$, temos que o contradomínio de todos os diferenciais são zero, logo, não pode ter diferenciais não nulos na sequência. Portanto, $E_{2}^{0, q}=0$, para todo $q>0$.

Por outro lado, $E_{2}^{0, q}=R^{0}\left(i^{*}\right) \circ R^{q}\left(i_{*}\right)=i^{*} \circ R^{q}\left(i_{*}\right)=i^{*}\left(\mathcal{H}^{q}\right)$. Então 


$$
\left(\mathcal{H}^{q}(A)\right)^{++}=i^{*}\left(\mathcal{H}^{q}(A)\right)=i^{*}\left(E_{2}^{0, q}\right)=0 \text {, para todo } q>0,
$$

Pela proposição $2.2 .21,\left(\mathcal{H}^{q}(A)\right)^{+}$é separável, logo, o mapa canônico $\left(\mathcal{H}^{q}(A)\right)^{+} \rightarrow$ $\left(\mathcal{H}^{q}(A)\right)^{++}$é mono.

Portanto, $\left(\mathcal{H}^{q}(A)\right)^{+}=0$ para todo $q>0$.

Vamos dar início à construção de um complexo que ainda não será uma generalização do Čech mas no auxiliará para este seja definido adiante.

Na proposição não precisamos, mas no que se segue devemos considerar $\mathcal{C}$ uma categoria com pullbacks (observamos que todo topos de Grothendieck é determinado com algum casal $(\mathcal{C}, J)$ com essa propriedade adicional).

No caso dos feixes tínhamos uma cobertura de um espaço topológico, então o primeiro passo é considerar um cobertura que faça sentido em $\mathcal{C}$. Também tínhamos definido $U_{i_{0}, \ldots, i_{q}}$ por intersecções, o análogo para o topos de Grothendieck é considerar o pullback. Seja $\mathcal{U}=\left\{U_{i} \rightarrow U\right\}$ família de morfismos em $\mathcal{C}$, defina $U_{i_{0}, \ldots, i_{q}}=U_{i_{0}} \times_{U} \ldots \times_{U} U_{i_{q}}$. Assim obteremos uma sequência em $P=$ Sets $^{\mathcal{C}^{o p}}$, dada por projeções e propriedades universais, da forma:

$$
\ldots \rightleftharpoons \coprod_{i_{0}<i_{1}<i_{2}} h_{U_{i_{0}, i_{1}, i_{2}}} \longrightarrow \coprod_{i_{0}<i_{1}} h_{U_{i_{0}, i_{1}}} \longrightarrow h_{U}
$$

onde $h_{U}=H o m_{\mathcal{C}}(-, U)$ é o funtor representável e temos morfismos do $U_{i_{0}, \ldots, i_{q}} \stackrel{\delta_{k}}{\longrightarrow}$ $U_{i_{0}, \ldots, \hat{i_{k}}, \ldots i_{q}}$ que esquecem $i_{k}$.

Aplicando o funtor livre $Z: P \rightarrow \mathbf{A b}(P)$, que é adjunto à esquerda do funtor esquecimento $U: \mathbf{A b}(P) \rightarrow P$, obtemos uma sequência em $\mathbf{A} \mathbf{b}(P)$ da forma

$$
\cdots \Longrightarrow \coprod_{i_{0}<i_{1}<i_{2}} Z\left(h_{U_{i_{0}, i_{1}, i_{2}}}\right) \Longrightarrow \coprod_{i_{0}<i_{1}} Z\left(h_{U_{i_{0}, i_{1}}}\right) \longrightarrow Z\left(h_{U}\right)
$$

Lembre que $Z$, por ser adjunto à esquerda, fornece isos do tipo $Z\left(\coprod_{i \in I}\right) h_{U_{i}} \cong \coprod_{i \in I} Z\left(h_{U_{i}}\right)$

Tal sequência determina um complexo de cadeias, denotado por $N_{\bullet}(\mathcal{U})$ com operador de bordo

$$
\left(d_{p} \alpha\right)=\sum_{k=0}^{q+1}(-1)^{k} \alpha\left(\delta_{k}\right)_{\left.\right|_{U_{0}, \ldots, i_{q+1}}}
$$

Proposição 3.4.11 A sequência $\cdots \rightarrow N_{2}(U) \rightarrow N_{1}(U) \rightarrow N_{0}(U)$ é exata em $\boldsymbol{A} \boldsymbol{b}(P)$

\section{Demonstração:}

Uma vez que $P=S e t s^{\mathcal{C}^{o p}}$, para provar que essa sequência exata basta verificar que $\cdots \rightarrow N_{2}(U)(V) \rightarrow N_{1}(U)(V) \rightarrow N_{0}(U)(V)$ é exata em $\mathbf{A b}($ Sets $)$ para $V$ objeto de $\mathcal{C}$.

Note que o funtor de avaliação em $V$ é um funtor de imagem inversa e que comuta com o funtor livre $Z$.

Então $N_{q}(U)(V)$ é um grupo abeliano livre gerado por $\underset{i_{0}<\ldots<i_{p}}{\amalg} H o m_{\mathcal{C}}\left(V, U_{i_{0}, \ldots, i_{p}}\right)$.

Sejam $\psi: V \rightarrow U$ e $\theta_{i}: U_{i} \rightarrow U$ morfismos em $\mathcal{C}$, definimos

$$
S_{\psi}=\coprod_{i \in I} \operatorname{Hom}_{\mathcal{C} \downarrow U}\left(\psi, \theta_{i}\right)
$$

Usando o pullback de $U_{i_{0}, \ldots, i_{p}}$ obtemos

$$
\coprod_{i_{0}<\ldots<i_{p}} \operatorname{Hom}_{\mathcal{C}}\left(V, U_{i_{0}, \ldots, i_{p}}\right) \cong \coprod_{\psi \in H o m(V, U)} S_{\psi}^{p+1}
$$

Como $A b($ Sets $) \cong A b$ satisfaz $\mathrm{AB} 4$ temos que o coproduto de sequências exatas é exata, logo, é suficiente mostrar que $\cdots \rightarrow Z\left(S_{\psi}^{3}\right) \rightarrow Z\left(S_{\psi}^{2}\right) \rightarrow Z\left(S_{\psi}\right)$ é exata. 
Os mapas $d: Z\left(S_{\psi}^{q+1}\right) \rightarrow Z\left(S_{\psi}^{q}\right)$ são a soma alternada dos mapas induzidos pelas projeções $S_{\psi}^{q+1} \rightarrow S_{\psi}^{q}$.

Se $S_{\psi}=\emptyset$ nada a fazer.

Se $S_{\psi} \neq \emptyset$, tome $t \in S_{\psi}$ e considere o homomorfismo $Z\left(S_{\psi}^{q}\right) \rightarrow Z\left(S_{\psi}^{q+1}\right)$ induzido por $h\left(\left(s_{1}, \ldots, s_{q}\right)\right)\left(t, s_{1}, \ldots, s_{q}\right)$, onde $s_{1}, \ldots, s_{q} \in S_{\psi}$. Indicando as contas, temos:

$$
\begin{aligned}
(h \circ d)\left(s_{1}, \ldots, s_{q}\right) & =h\left(\left(s_{2}, \ldots, s_{q}\right)-\left(s_{1}, s_{3}, \ldots, s_{q}\right)+\ldots+(-1)^{q}\left(s_{1}, \ldots, s_{q-1}\right)\right) \\
& =\left(t, s_{2}, \ldots, s_{q}\right)-\left(t, s_{1}, s_{3}, \ldots, s_{q}\right)+\ldots+(-1)^{q}\left(t, s_{1}, \ldots, s_{q-1}\right)
\end{aligned}
$$

Do outro lado:

$$
\begin{aligned}
(d \circ h)\left(s_{1}, \ldots, s_{q}\right) & =d\left(\left(t, s_{1}, \ldots, s_{q}\right)\right) \\
& =\left(\left(s_{1}, \ldots, s_{q}\right)-\left(t, s_{2}, \ldots, s_{q}\right)+\left(t, s_{1}, s_{3}, \ldots, s_{q}\right)-\ldots+(-1)\left(t, s_{1}, \ldots, s_{q-1}\right)\right)
\end{aligned}
$$

Assim, $((h \circ d)+(d \circ h))\left(\left(s_{1}, \ldots, s_{q}\right)\right)=\left(s_{1}, \ldots, s_{q}\right)$

Como isso calculamos $d t+t d=i d$, isto é, $i d \sim 0$. Logo, pela Proposição 1.3.10, os grupos de homologia são zero, implicando que a sequência é exata.

Definição 3.4.12 Seja $A \in \mathbf{A b}(P)$, o complexo de cocadeias de Čech é dado por

$$
C^{q}(\mathcal{U}, A)=H_{\operatorname{Hom}} \mathbf{A b}\left(N_{q}(\mathcal{U}), A\right)
$$

com operadores de bordo sendo da forma $-\circ d_{p}$

Veja que, de fato, é complexo de cocadeia, pois $\left(-\circ d_{q+1}\right) \circ\left(-\circ d_{q}\right)=-\circ\left(d_{q} \circ d_{q+1}\right)=$ $-\circ 0=0$.

Esse complexo induz uma sequência

$$
\prod_{i_{0}} A\left(U_{i_{0}}\right) \stackrel{d^{0}}{\rightarrow} \prod_{i_{0}<i_{1}} A\left(U_{i_{0}, i_{1}}\right) \stackrel{d^{1}}{\rightarrow} \prod_{i_{0}<i_{1}<i_{2}} A\left(U_{i_{0}, i_{1}, i_{2}}\right) \stackrel{d^{2}}{\rightarrow} \ldots
$$

porque temos o seguinte diagrama comutativo, onde todas as flechas verticais são isomorfismos e vamos explicar o porquê.

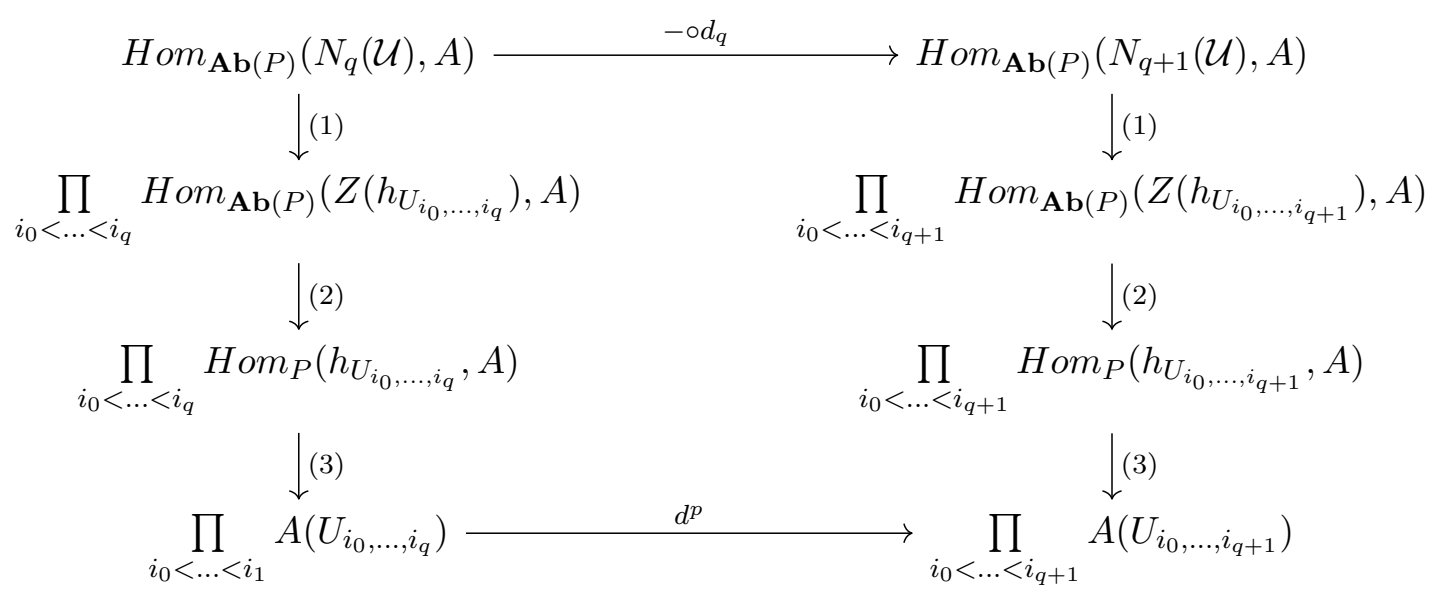

O isomorfismo (1) vale pois dados $X_{j}$ 's e $Y$ objetos de uma categoria $\mathcal{C}$, existem isomorfismos naturais $\operatorname{Hom}_{\mathcal{C}}\left(\coprod_{j \in J} X_{j}, Y\right) \cong \prod_{j \in J} \operatorname{Hom}_{\mathcal{C}}\left(X_{j}, Y\right)$.

O isomorfismo (2) vale por propriedade do funtor adjunto do funtor esquecimento $\mathbf{A b}(P) \rightarrow P$.

O isomorfismo (3) é uma aplicação do Lema de Yoneda. 
Definição 3.4.13 Seja $A$ um grupo abeliano em $P=S e t s^{\mathcal{C}^{o p}}$ e $\mathcal{U}$ uma família de morfismo em $\mathcal{C}$. Definimos o $q$-ésimo grupo de cohomologia de Čech de $\mathcal{U}$ com coeficientes em $A$ por $H^{q}(\mathcal{U}, A)=K e r d^{q} / I m d^{q-1}$

Note que essa definição é análoga à dos grupos de cohomologia de Čech em $S h_{A b}(X)$ $(3.4 .3)$

Proposição 3.4.14 $\mathrm{H}^{q}(U,-)$ é o q-ésimo funtor derivado à direita de $H^{0}(U,-)$

\section{Demonstração:}

Sabemos que $N_{\bullet}(U)$ é um complexo de cadeias $\ldots \longrightarrow Z\left(h_{U}\right)$

O complexo de cocadeias de Čech é $C^{q}(\mathcal{U}, A)=\operatorname{Hom}_{\mathbf{A b}(P)}\left(N_{q}(\mathcal{U}), A\right)$.

Pelo Lema 1.4.2, que $\operatorname{Hom}_{\mathbf{A b}(P)}(-, A)$ é exato se $A$ é injetivo, aplicando esse funtor exato na sequência exata dada na Proposição 3.4.11 obtemos que a sequência abaixo é exata.

$$
\operatorname{Hom}_{\mathbf{A b}(P)}\left(N_{0}(U), A\right) \longrightarrow \operatorname{Hom}_{\mathbf{A b}(P)}\left(N_{1}(U), A\right) \longrightarrow \ldots
$$

Ou seja, $C^{0}(U, A) \longrightarrow C^{1}(U, A) \longrightarrow \ldots$ é exata e $H^{q}(U, A)=0$ para todo $q>0$.

Veja que funtores representáveis são projetivos em $P$. De fato, seja $g: A \rightarrow B$ um morfismo sobrejetor em $P$ e $\gamma: h_{C}(-) \rightarrow B$. Pelo Lema de Yoneda, $\gamma \in \operatorname{Hom}\left(h_{C}, B\right)$ corresponde a $\bar{\gamma} \in B(C)$. Como $g$ é sobrejetor, $\bar{\gamma}=g(a)$ para $a \in A(C)$. Novamente pelo Lema de Yoneda, $a$ está em correspondência com $\bar{a} \in \operatorname{Hom}\left(h_{C}, A\right)$. Assim obtemos $\bar{a}: h_{C} \rightarrow A$ tal que $g \circ \bar{a}=\gamma$. Portanto, $h_{C}(-)$ é projetivo.

Então, aplicando apenas o funtor livre $Z$ obtemos que os $N_{q}(U)$ são projetivos em $\operatorname{Ab}(P)$ para todo $q \geq 0$. Segue da versão dual do Lema 1.4.2 que $\operatorname{Hom}_{\mathbf{A b}(P)}\left(N_{q}(\mathcal{U}),-\right)$ é exato, logo, o funtor que vai de $\mathbf{A b}(\mathcal{E})$ em $\mathbf{A b}(S e t s)$ por $A \mapsto C^{\bullet}(U, A)$ é exato.

Então

$$
0 \longrightarrow A \longrightarrow C^{0}(U, A) \longrightarrow C^{1}(U, A) \longrightarrow C^{2}(U, A) \longrightarrow \ldots
$$

é exata.

Pelo Teorema 1.4.12 concluímos que $R^{q}(C(U,-)(A)) \cong H^{q}\left(C^{\bullet}(U, A)\right)$, como desejado.

Considere duas famílias de morfismos $\mathcal{U}=\left\{U_{i} \rightarrow U \mid i \in I\right\}$ e $\mathcal{V}=\left\{V_{j} \rightarrow U \mid j \in J\right\}$. Elas cumprem em $S h(\mathcal{C}, J)$ o papel das coberturas por abertos de um espaço topológico $X$ em $S h(X)$. Assim como refinamos coberturas por aberto, também podemos refinar essas famílias.

Definição 3.4.15 Dizemos que $r: \mathcal{V} \rightarrow \mathcal{U}$ é um mapa de refinamento se $f: J \rightarrow I$ é uma função munida com uma família de fatorações

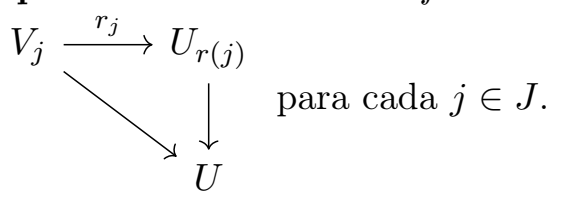

Dessa forma cada mapa de refinamento $r: \mathcal{V} \rightarrow \mathcal{U}$ induz um morfismo $V_{j} \rightarrow U_{r(j)}$ sobre $U$ e obtemos morfismo de cadeias $N_{\bullet}(\mathcal{V}) \rightarrow N_{\bullet}(\mathcal{U})$. isto é,

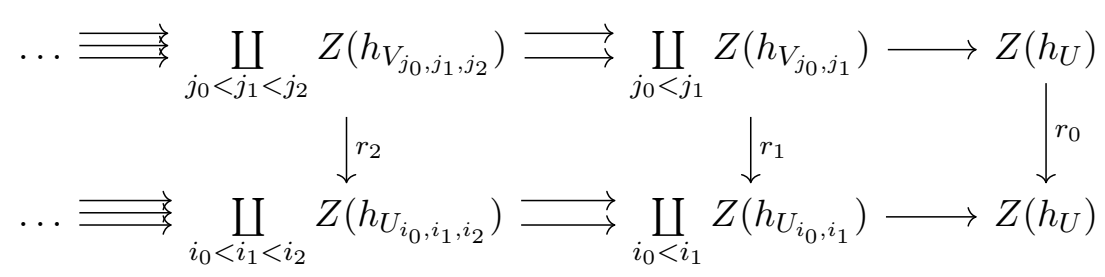


Observe que se $R$ é um crivo de $U$ gerado pela família $\mathcal{U}$, então o mapa inclusão $\mathcal{U} \rightarrow R$ é um mapa de refinamento.

Proposição 3.4.16 Sejam $r$ e $s$ mapas de refinamento $\mathcal{V} \rightarrow \mathcal{U}$. Então os mapas de cadeia r. e s. são homotópicos por cadeias.

\section{Demonstração:}

Devemos encontrar uma sequência de morfismos $N_{q}(\mathcal{V}) \rightarrow N_{q+1}(\mathcal{U})$ que torna $r_{\bullet}$ e $s_{\bullet}$ homotópicos por cadeia.

Sejam $i_{0}, \ldots, i_{q} \in I$, chame $\sigma=\left(i_{0}, \ldots, i_{q}\right)$. Para cada $l \in\{0,1, \ldots, q\}$ definimos um morfismo sobre $U$

$$
t_{\sigma}^{l}=\left(r_{i_{0}}, \ldots, r_{i_{l}}, s_{i_{l}}, \ldots, s_{i_{q}}\right): V_{\sigma} \rightarrow U_{\left(r_{i_{0}}, \ldots, r_{i_{l}}, s_{i_{l}}, \ldots, s_{i_{q}}\right)}
$$

Esse morfismo induz um homomorfismo de grupos $t_{q}^{l}: N_{q}(\mathcal{V}) \rightarrow N_{q+1}(\mathcal{U})$. Usaremos uma soma alternada de $t_{q}^{l}$ para montar a homotopia de cadeia.

Essas contas são mais complicadas que de costume, vamos ver o que acontece quando $q=1$.

$$
\sigma=\left(i_{0}, i_{1}\right), \quad t_{\sigma}^{0}=\left(r_{i_{0}}, s_{i_{0}}, s_{i_{1}}\right) \quad t_{\sigma}^{1}=\left(r_{i_{0}}, r_{i_{1}}, s_{i_{1}}\right)
$$

A soma alternada é $t_{1}^{(l)}=\sum_{l=0}^{1}(-1)^{l+1} t_{1}^{l}=-t_{q}^{0}+t_{q}^{1}=-\left(r_{i_{0}}, s_{i_{0}}, s_{i_{1}}\right)+\left(r_{i_{0}}, r_{i_{1}}, s_{i_{1}}\right)$.

Estamos com um diagrama não comutativo da forma

$$
\begin{aligned}
& \ldots \stackrel{d_{3}^{\mathcal{V}}}{\longrightarrow} \coprod_{i_{0}<i_{1}<i_{2}} Z\left(h_{V_{i_{0}, i_{1}, i_{2}}}\right) \stackrel{d_{2}^{\mathcal{\nu}}}{\longrightarrow} \underset{i_{0}<i_{1}}{\coprod_{1}} Z\left(h_{V_{i_{0}, i_{1}}}\right) \stackrel{d_{1}^{\mathcal{V}}}{\longrightarrow} \ldots
\end{aligned}
$$

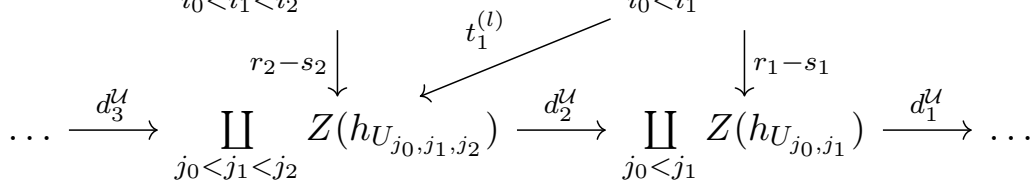

Os índices $j$ são obtidos através dos refinamentos $r$ e $s$.

Chame $\tau=\left(i_{0}, i_{1}, i_{2}\right)$ e $\alpha_{\tau}: Z\left(h_{V_{\tau}}\right) \rightarrow \coprod_{\tau} Z\left(h_{V_{\tau}}\right)$. Seja $C$ objeto, considere um elemento $\theta_{\tau} \in Z\left(h_{V_{\tau}}(C)\right)$, temos

$$
\left(r_{2}-s_{2}\right) \circ \alpha_{\tau}\left(\theta_{\tau}\right)=\theta_{r_{i_{0}}, r_{i_{1}}, r_{i_{2}}}
$$

Por outro lado

$$
d_{2}^{\mathcal{V}}\left(\alpha_{\tau}\left(\theta_{\tau}\right)\right)=\theta_{i_{0}, i_{1}}-\theta_{i_{0}, i_{2}}+\theta_{i_{1}, i_{2}}
$$

Diagramaticamente

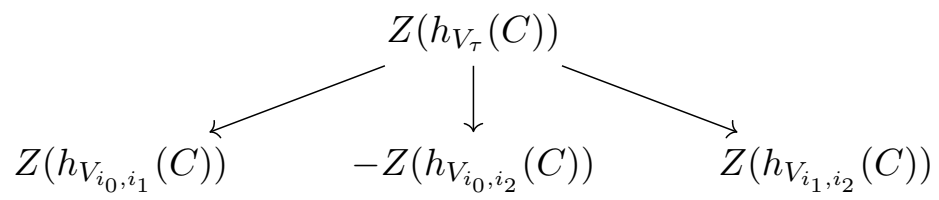

Então aplicamos $t_{1}^{(l)}$

$$
\begin{aligned}
t_{1}^{(l)} \circ d_{2}^{\mathcal{V}}\left(\alpha_{\tau}\left(\theta_{\tau}\right)\right) & =t_{1}^{(l)}\left(\theta_{i_{0}, i_{1}}-\theta_{i_{0}, i_{2}}+\theta_{i_{1}, i_{2}}\right) \\
& =-\theta_{r_{i_{0}}, s_{i_{0}}, s_{i_{1}}}+\theta_{r_{i_{0}}, r_{i_{1}}, s_{i_{1}}} \\
& +\left(-\theta_{r_{i_{0}}, s_{i_{0}}, s_{i_{2}}}+\theta_{r_{i_{0}}, r_{i_{2}}, s_{i_{2}}}\right) \\
& +\left(-\theta_{r_{i_{1}}, s_{i_{1}}, s_{i_{2}}}+\theta_{r_{i_{1}}, r_{i_{2}}, s_{i_{2}}}\right)
\end{aligned}
$$


Agora veremos como fica $d_{3}^{\mathcal{U}} \circ t_{2}^{(l)}$.

$$
\begin{aligned}
d_{3}^{\mathcal{U}} \circ t_{2}^{(l)}\left(\alpha_{\tau}\left(\theta_{\tau}\right)\right) & =d_{3}^{\mathcal{U}}\left(-\theta_{r_{i_{0}}, s_{i_{0}}, s_{i_{1}}, s_{i_{2}}}+\theta_{r_{i_{0}}, r_{i_{1}}, s_{i_{1}}, s_{i_{2}}}-\theta_{r_{i_{0}}, r_{i_{1}}, r_{i_{2}}, s_{i_{2}}}\right) \\
& =-\left(\theta_{s_{i_{0}}, s_{i_{1}}, s_{i_{2}}}-\theta_{r_{i_{0}}, s_{i_{1}}, s_{i_{2}}}+\theta_{r_{i_{0}}, s_{i_{0}}, s_{i_{2}}}-\theta_{r_{i_{0}}, s_{i_{0}}, s_{i_{1}}}\right) \\
& +\left(\theta_{r_{i_{1}}, s_{i_{1}}, s_{i_{2}}}-\theta_{r_{i_{0}}, s_{i_{1}}, s_{i_{2}}}+\theta_{r_{i_{0}}, r_{i_{1}}, s_{i_{2}}}-\theta_{r_{i_{0}}, r_{i_{1}}, s_{i_{1}}}\right) \\
& -\left(\theta_{r_{i_{1}}, r_{i_{2}}, s_{i_{2}}}-\theta_{r_{i_{0}}, r_{i_{2}}, s_{i_{2}}}+\theta_{r_{i_{0}}, r_{i_{1}}, s_{i_{2}}}-\theta_{r_{i_{0}}, r_{i_{1}}, r_{i_{2}}}\right)
\end{aligned}
$$

Fazendo

$$
\begin{aligned}
d_{3}^{\mathcal{U}} \circ t_{2}^{(l)}\left(\alpha_{\tau}\left(\theta_{\tau}\right)\right)+t_{1}^{(l)} \circ d_{2}^{\mathcal{V}}\left(\alpha_{\tau}\left(\theta_{\tau}\right)\right) & =\theta_{r_{i_{0}}, r_{i_{1}}, r_{i_{2}}} \\
& =\left(r_{2}-s_{2}\right) \circ \alpha_{\tau}\left(\theta_{\tau}\right)
\end{aligned}
$$

Assim, nesse caso, a homotopia de cadeias está provada. Para $q \geq 0$ em geral usamos $\tau=\left(i_{0}, \ldots, i_{q}\right)$ e $t_{q}^{(l)}=-\left(r_{i_{0}}, s_{i_{0}}, \ldots, s_{i_{q}}\right)+\ldots+\left(r_{i_{0}}, \ldots, r_{i_{q}}, s_{i_{q}}\right)$.

Desenvolvendo as contas de forma análoga obtemos, como desejado,

$$
\left(r_{q}-s_{q}\right)\left(\alpha_{\tau}\left(\theta_{\tau}\right)\right)=d_{q+1}^{\mathcal{U}} \circ t_{q}^{(l)}\left(\alpha_{\tau}\left(\theta_{\tau}\right)\right)+t_{q-1}^{(l)} \circ d_{q}^{\mathcal{V}}\left(\alpha_{\tau}\left(\theta_{\tau}\right)\right)
$$

Usamos o resultado acima para mostrar que existe um isomorfismo entre os grupos de cohomologias em uma família de morfismos e os grupos de cohomologias no crivo gerado por essa família.

Proposição 3.4.17 Seja $\mathcal{U}=\left\{U_{i} \rightarrow U \mid i \in I\right\}$ família de morfismos e $R$ o crivo gerado por $\mathcal{U}$. Então $\mathrm{H}^{q}(\mathcal{U}, A) \cong \mathrm{H}^{q}(R, A)$ é isomorfismo para qualquer $A \in A b(\mathcal{E})$.

\section{Demonstração:}

$R$ ser um crivo gerado por $\mathcal{U}$ significa que qualquer morfismo $\alpha$ em $R$ se fatora por $U_{i}$, isto é, $\alpha=\gamma_{i} \circ \beta_{j}$, onde $\beta_{j}: V_{j} \rightarrow U_{i}$ e $\gamma_{i} \in \mathcal{U}$. Então existe um mapa de refinamento $R \rightarrow \mathcal{U}$.

Por outro lado, a inclusão $\mathcal{U} \rightarrow R$ também nos fornece um mapa de refinamento.

Usando a Proposição 3.4.16 temos que esses refinamentos são único a menos de homotopia por cadeia, porém, inversos. Isso nos dá que $N_{\bullet}(\mathcal{U})$ e $N_{\bullet}(R)$ são homotópicos, logo, $C^{\bullet}(\mathcal{U}, A)$ e $C^{\bullet}(\mathcal{U}, A)$ também são e, portanto, os grupos de cohomologia são isomorfos (Proposição 1.3.10).

Além disso, esse isomorfismo não depende da escolha do refinamento $R \rightarrow \mathcal{U}$, pois - pela Proposição 3.4.16 - duas escolhas distintas de refinamento resultam em mapas de cocadeia que diferem apenas por homotopia, logo, ao passar para os grupos de cohomologia obtemos isomorfismos.

Agora vamos fornecer uma outra definição da Cohomologia de Čech, mas em vez de considerarmos a famílias de morfismos em $\mathcal{C}$, sobre um objeto $U$ teremos direto o objeto $U$ de $\mathcal{C}$.

Definição 3.4.18 Sejam $(\mathcal{C}, J)$ um casal onde $\mathcal{C}$ possui pullbacks, $U$ um objeto de $\mathcal{C}$ e $A$ pré-feixe de grupos abelianos em $\mathcal{C}$. Definimos o $q$-ésimo grupo de cohomologia de Čech de $U$ com coeficientes em $A$ como

$$
\check{\mathrm{H}}^{q}(U, A)=\underset{R \in J(U)}{\lim _{R \in}} H^{q}(R, A)
$$

Observação 3.4.19 Note que $\check{\mathrm{H}}^{0}(U, A) \cong \underset{R \in J(U)}{\lim _{\overparen{E}}} \operatorname{Hom}_{\mathcal{E}}(R, A)=A^{+}(U)$. 
Então o q-ésimo funtor derivado de + é um funtor $\mathbf{A b}(P) \rightarrow \mathbf{A b}(P)$ que leva um pré-feixe $A$ e outro pré-feixe mas da forma $U \mapsto \check{\mathrm{H}}^{q}(U, A)$, sendo $\check{\mathrm{H}}^{q}(U, A)$ funtorial em $U$.

Teorema 3.4.20 Seja $\mathcal{E}=S h(\mathcal{C}, J)$, $U$ objeto de $\mathcal{C}$ e $A \in A b(\mathcal{E})$. Então existe um homomorfismo $\check{\mathrm{H}}^{q}(U, A) \rightarrow \mathrm{H}^{q}(\mathcal{E}, l(U) ; A)$, onde l é o funtor canônico l $: \mathcal{C} \rightarrow S h(\mathcal{C}, J)$ que é iso quando $q=0,1$ e mono caso $q=2$.

\section{Demonstração:}

Seja $+: P \rightarrow P$ o funtor de "semi-feixificação", trabalharemos com a sequência espectral da composição $+\circ i_{*} \cong i_{*}$, onde $i: S h(\mathcal{C}, J) \rightarrow \operatorname{Sets}^{\mathcal{C}^{o p}}$

$$
\left(\mathbf{A b}(\mathcal{E}) \stackrel{i_{*}}{\rightarrow} \mathbf{A b}(P) \stackrel{+}{\rightarrow} \mathbf{A b}(P)\right)=\left(\mathbf{A b}(E) \stackrel{i_{*}}{\rightarrow} \mathbf{A b}(P)\right)
$$

Pelo Teorema 1.6.6, $E_{2}^{p, q} \cong\left(R^{p} \circ+\right)\left(R^{q} i_{*}\right)=\left(R^{p} \circ+\right)\left(\mathcal{H}^{q}\right)$. Aplicando o funtor exato que avalia em $U$ na composição $+\circ i_{*} \cong i_{*}$ obtemos $E_{2}^{p, q}=\check{\mathrm{H}}^{p}\left(U, \mathcal{H}^{q}(A)\right)$, pois $\mathcal{H}^{q}(A) \in \mathbf{A b}(P)$ e usamos a Observação 1.4.19 acima.

Ainda por 1.6.6, $E_{\infty}^{p, q}$ é filtração de $R^{p+q}\left(+\circ i_{*}\right)(-)$. Então é filtração do $(p+q)$-ésimo funtor derivado à direita de

$$
\begin{aligned}
\left(i_{*}(-)\right)^{+}(U) & \cong \underset{R \in \lim _{R(U)}}{ } \operatorname{Hom}_{P}\left(R, i_{*}(-)\right) \text { por propriedade de feixe } \\
& \cong \operatorname{Hom}_{P}\left(h_{U}, i_{*}(-)\right) \\
& \cong \operatorname{Hom}_{\mathcal{E}}(l(U),(-)) \\
& \cong H^{0}(\mathcal{E}, l(U) ; A) .
\end{aligned}
$$

Note que

$$
E_{2}^{p, 0}=R^{p}\left(\mathcal{H}^{0}(A)^{+}\right)=R^{p}\left(A^{+}\right)=R^{p}\left(\check{\mathrm{H}}^{0}(U, A)\right)=\check{\mathrm{H}}^{p}(U, A)
$$

Assim obtemos (aplicando a Proposição 1.6.7)

$$
\check{\mathrm{H}}^{p}(U, A)=E_{2}^{p, 0} \rightarrow E_{\infty}^{p, 0} \longmapsto H^{p}(\mathcal{E}, l(U) ; A)=R^{p+0}\left(H^{0}(\mathcal{E}, l(U) ; A)\right)
$$

Por outro lado, pela Proposição 3.4.10,

$$
E_{2}^{0, q}=\check{\mathrm{H}}^{0}\left(U, \mathcal{H}^{q}(A)\right)=\left(\mathcal{H}^{q}(A)\right)^{+}(U)=0 \text {, para todo } q>0
$$

Desta forma, $E_{2}^{p, 0} \rightarrow E_{\infty}^{p, 0}$ é iso quando $p=0,1$ ou 2 , pois esses grupos não podem ser atingidos por diferenciais não nulos

$$
\begin{aligned}
& E_{2}^{-2,1}=0 \longrightarrow E_{2}^{0,0} \\
& E_{2}^{-1,1}=0 \longrightarrow E_{2}^{1,0} \\
& E_{2}^{0,1}=0 \longrightarrow E_{2}^{2,0}
\end{aligned}
$$

Isso dá automaticamente que $\check{\mathrm{H}}^{p}(U, A) \cong E_{\infty}^{p, 0} \longmapsto \mathrm{H}^{p}(\mathcal{E}, l(U) ; A)$ é mono para $p=0,1$ ou 2. Para os casos $p=0$ ou 1 temos também que $E_{\infty}^{p, 0}$ é o único termo não nulo da filtração (se $p=2$ caimos numa filtração no tipo $0=F^{3} R^{2} \subseteq \ldots \subseteq F^{0} R^{2}$ e $F^{0} R^{2} / F^{1} R^{2}$ não é nulo), $\operatorname{logo}, E_{\infty}^{p, 0} \longmapsto \mathrm{H}^{p}(\mathcal{E}, l(U) ; A)$ também é iso.

Gostaríamos que o resultado fosse verdade para todo $q>0$. Veremos que de fato é se fizermos algumas outras imposições.

Proposição 3.4.21 Seja $\mathcal{E}=S h(\mathcal{C}, J)$ e $A \in A b(\mathcal{E})$. Suponha que exista um subconjunto $K$ dos objetos de $\mathcal{C}$ tal que: 
(i) Para cada $V \in K$, temos $\check{\mathrm{H}}^{q}(V, A)=0, \forall q>0$

(ii) Para cada objeto $U$ de $\mathcal{C}$ existe $J$-cobertura $\left\{V_{i} \rightarrow U \mid i \in I\right\}$ com cada $V_{i}$ em $K$.

(iii) Se $V$ e $W$ estão em $K$, então qualquer pullback $V \times{ }_{U} W$ está em $K$.

Então o mapa canônico $\check{\mathrm{H}}^{q}(U ; A) \rightarrow \mathrm{H}^{q}(\mathcal{E}, l(U) ; A)$ é iso para todo objeto $U$ de $\mathcal{C}$ e para todo $q>0$.

\section{Demonstração:}

Seja $\mathcal{U}=\left\{U_{j} \rightarrow U \mid j \in J\right\}$ uma $J$-cobertura (no sentido de 2.2.9). Por definição de cobertura e pela propriedade $(i i)$ acima, podemos refinar $\mathcal{U}$ para uma cobertura $\mathcal{V}=$ $\left\{V_{j i} \rightarrow U \mid i \in I\right\}$ onde cada $V_{j i}$ pertence a $K$.

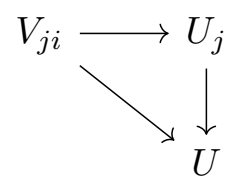

Vamos chamar $V_{i}=V_{j i}$ por ser mais prático e nesse contexto não provocar confusão.

Com o que fizemos temos um refinamento $r: \mathcal{V} \rightarrow \mathcal{U}$. Note que cada elemento de $\check{\mathrm{H}}^{q}(U ; A)$ vem de um elemento de $H^{q}(\mathcal{V}, A)$, pois $H^{q}(\mathcal{V}, A) \cong H^{q}(R, A)$ onde $R$ é crivo gerado por $\mathcal{V}$ e $\check{\mathrm{H}}^{q}(U ; A)=\underset{R \in J(U)}{\lim _{R}} H^{q}(R, A)$.

Vimos que $V_{i}$ está em $K$ para todo $i \in I$. Pelo item $(i i i), V_{0} \times_{U} \ldots \times_{U} V_{q}$ está em $K$

Feitas essas observações vamos provar o resultado por indução em $q$. Se $q=0$, basta aplicar o Teorema 3.4.20. Suponha que vale para todo $q<n$ com $n>1$ fixado.

Considere o complexo $C^{\bullet}\left(\mathcal{V}, \mathcal{H}^{q}(A)\right), \mathcal{H}^{q}(A)=R^{q} i_{*}(A)$ e $\mathcal{V}$ é a família construída acima.

Aplicamos a Proposição 3.3.10 no morfismo geométrico $i: \mathcal{E} \rightarrow P$ e temos que $\mathcal{H}^{q}(A)$ é $U \mapsto H^{q}(\mathcal{E}, l(U) ; A)$. Por hipótese de indução, $H^{q}(\mathcal{E}, l(U) ; A) \cong \check{\mathrm{H}}^{q}(U ; A)$, então $\mathcal{H}^{q}(A)$ é o funtor $U \mapsto \check{\mathrm{H}}^{q}(U ; A)$. Como $V_{0} \times_{U} \ldots \times_{U} V_{q}$ está em $K$, pelo item $(i)$, temos $\check{\mathrm{H}}^{0}\left(V_{0} \times_{U}\right.$ $\left.\ldots \times{ }_{U} V_{q} ; A\right)=0$ para todo $p>0$.

Agora observe que todos os objetos avaliados em $\mathcal{H}^{q}(A)$ são da forma $V_{0} \times_{U} \ldots \times_{U} V_{q} \in K$, $\left.\operatorname{logo}, C^{p}(\mathcal{V}), \mathcal{H}^{q}(A)\right)=\operatorname{Hom}_{\mathbf{A b}(P)}\left(N_{q}\left(\mathcal{V}, \mathcal{H}^{q}(A)\right)\right.$.

Usando a Proposição 3.4.17 obtemos

$$
0=H^{p}\left(\mathcal{V}, \mathcal{H}^{q}(A)\right) \cong H^{p}\left(R, \mathcal{H}^{q}(A)\right)
$$

De onde segue

$$
0=\underset{R \in J(U)}{\lim _{R \in J}} H^{q}\left(R, \mathcal{H}^{q}(A)\right)=\check{\mathrm{H}}^{q}\left(U ; \mathcal{H}^{q}(A)\right)
$$

$\check{\mathrm{H}}^{q}\left(U ; \mathcal{H}^{q}(A)\right)=0$ significa, pela sequência espectral vista na Proposição 3.4 .20 , que $E_{r}^{p, q}=$ 0 para todo $p>0$ e para todo $0<q<n$.

Vimos que $E_{2}^{0, q}=0$ para todo $q>0$, então $E_{2}^{0, n}=0$.

Observe

$$
\begin{array}{ccc}
E_{2}^{p, q} & E_{2}^{p+1, q} & \\
E_{2}^{p, q-1} & E_{2}^{p+1, q-1} & \\
& \\
E_{2}^{p, 0} & \cdots & E_{2}^{n, 0}
\end{array}
$$

Agrupando o que sabemos, $E_{2}^{p, 0}, 0 \leq p \leq n$ são as únicas entradas não nulas na diagonal $p+q=n$ ou abaixo dela. Como os grupos só podem ser atingidos por diferenciais não 
nulos, temos que $E_{2}^{n, 0} \longmapsto E_{\infty}^{n, 0}$ é iso. Novamente, como $E_{\infty}^{n, 0}$ é o único termo não nulo da filtração, $E_{\infty}^{n, 0}=\check{\mathrm{H}}^{n}(U ; A) \rightarrow \mathrm{H}^{n}(\mathcal{E}, l(U) ; A)$ é iso.

Assim concluímos que o resultado vale também para $q=n$.

Vamos dar um exemplo de aplicação da Proposição acima.

Seja $X$ um aberto de $\mathbb{R}^{n}$ e $K=\{U \subseteq X \mid U$ é aberto e convexo $\}$ e $A$ um feixe em $X$. A propriedade $(i)$ vale pois todo conjunto convexo não vazio é contrátil, logo, $\check{\mathrm{H}}^{n}(U ; A)=0$ para todo $U \in K$ e $q>0$. Não falamos sobre isso aqui, mas em topologia algébrica é bem conhecido que espaço contráteis resultam em grupos de (co)homologia nulos. Para verificar (ii) basta pegar as bolas abertas, pois são também convexas. Por fim, para (iii) usamos que insterscção de convexos é convexo.

Uma vez que todas as três condições são satisfeitas aplicamos a proposição e obtemos que a noção usual dos grupos de cohomologia coincide com o grupos de cohomologia de Čech.

Com isso encerramos nossa apresentação de como aplicar as ferramentas de Álgebra Homológica em Topos de Grothendieck. Deixamos algumas comparações entre a conhecida Cohomologia de feixes e a Cohomologia dos Topos de Grothendieck para o capítulo seguinte, assim como outras ideias de estiverem presentes ao longo dessa Dissertação de Mestrado e caminhos para pesquisas futuras. 


\section{Capítulo 4}

\section{Considerações Finais}

Os teoremas fundamentais dessa Dissertação são o 3.3.4 e o 3.3.8. No primeiro garantimos que $\mathbf{A b}(\mathcal{E})$ é uma categoria abeliana para qualquer topos $\mathcal{E}$. Graças a isso temos a esperança de desenvolver uma teoria de cohomologia para os topos, esperança essa que se concretiza através do 3.3.8 com o topos mais esperado possível, o de Grothendieck, pois ganhamos a propriedade de $\mathbf{A b}(\mathcal{E})$ possuir suficientes injetivos.

A centralidade desses resultados nos leva a fazer outras perguntas:

- Quais as propriedades de uma categoria abeliana da forma $\mathbf{A b}(\mathcal{E})$, para $\mathcal{E}$ um topos (respectivamente, um topos de Grothendieck)?

- Dada uma categoria abeliana $\mathscr{C}$, em que condições suficientes existe um topos $\mathcal{E}$ tal que $\mathscr{C} \simeq \operatorname{Ab}(\mathcal{E})$ ?

- Para que outras categorias $\mathcal{C}$ temos que $\mathbf{A b}(\mathcal{C})$ é abeliana ou $\mathrm{AB} 5$ ?

Não encontramos respostas satisfatórias a essas questões na literatura. Acreditamos que uma possível alternativa para compreendê-las melhor seja através de uma comparação mais aprofundada entre categorias abelianas e topos, eventualmente, voltando mais a atenção para as lógicas envolvidas e como a teoria de modelos pode ser usada para descrever a categoria dos grupos abelianos internos.

Escolhemos apresentar $\mathbf{A b}(\mathcal{E})$ através dos objetos de grupos por ser a forma mais simples de compreender a estrutura da categoria, mas pensamos que a abordagem via modelos seja mais interessante para responder as questões mencionadas acima pois mais informações sobre o funtor esquecimento devem surgir nesse caso. A ideia é que $\mathbf{A b}(\mathcal{E})$ pode ser visto como um $A b$-modelo em $\mathcal{E}$ e com isso podemos aproveitar propriedade já conhecidas do funtor esquecimento vistos em teorias algébricas como feito no Capítulo 3 de [Bor94b]. Seguindo esse mesmo espírito, verificar se existe relação entre as categoria internas como na Definição 2.4.23 e a definição de $\mathbf{A b}(\mathcal{E})$ que fornecemos pode ser útil.

Aproveitando a centralidade de termos $\mathbf{A b}(\mathcal{E})$ categoria abeliana com suficientes objetos injetivos para realizarmos a cohomologia, será que também teríamos $\mathbf{A b}(\mathcal{E})$ com suficientes objetos projetivos e assim poderíamos pensar na homologia? O Teorema 8.13 de [Joh14] prova justamente a existência dos suficientes injetivos e logo após encerrar a demonstração, comenta que a falha do axioma da escolha para um topos qualquer implica que em geral $\mathcal{E}$ não possui suficientes projetivos e, dessa forma, $\mathbf{A b}(\mathcal{E})$ também não possui. Essa discussão é ainda mais interessante se considerarmos o artigo [Bla83] onde temos que o axioma da escolha é equivalente à cohomologia ser trivial, isto é, $H^{1}(X ; G)=0$, para todo espaço discreto $X$ e todos os grupos $G$. Com isso surge outra questão a ser explorada: as relações a ausência de projetivos e a não-trivilidade de $H^{1}$.

Voltando para as semelhanças entre topos e categorias abelianas vamos organizar algumas na seguinte tabela, a coluna esquerda contém o resultado em si e as outras duas colunas estão indicadas onde é possível encontrar a demonstração para as categorias abelianas e para os topos 


\begin{tabular}{l|l|l} 
& Caterogias Abelianas & Topos Elementares \\
\hline Todo mono é equalizador & Definição 1.1.13(2) & Proposição 2.3.10 \\
Todo epi é coequalizador & Definição 1.1.13(2) & Corolário 2.4.15 \\
Mono + Epi = Iso & Proposição 1.1.14 & Corolário 2.3.11 \\
Finitamente Completa & Proposição 1.1.17 & Definição 2.3.6(1) \\
Finitamente Cocompleta & Proposição 1.1.17 & Observação 2.3.7 \\
Interseç̧ão de Subobjetos existe & Proposição 1.1.16 & Lema 2.5.5 \\
Fatoração em epi e mono & Teorema 1.1.19 & Teorema 2.4.14
\end{tabular}

Existem outras propriedades em comum, mas essas e outras se devem ao que comentamos na Seção 6 do Capítulo 2: ambas são categorias regulares. Por exemplo, como demonstrado no Teorema 2.1.3 de [Bor94b], toda categoria regular admite fatoração em epi e mono. As categorias regulares nos dão resultados mais gerais do que o apresentamos. Comentamos logo no início do Capítulo 3 que $\mathbf{A b}(\mathcal{E})$ poderia ser visto como um modelo de uma teoria algébrica, nesse caso Ab seria uma teoria algébrica finitária. Em [Bar71] Teorema 5.11, página 24, temos:

Seja $\mathcal{C}$ uma categoria regular e $T$ uma teoria algébrica finitária, então $T(\mathcal{C})$ é regular e $T(\mathcal{C}) \rightarrow \mathcal{C}$ é funtor exato e reflete isomorfismos.

Assim perdemos algumas especificidades, aplicando esse resultado teríamos direto que $\operatorname{Ab}(\mathcal{E})$ é uma categoria regular, mas não teríamos que é uma categoria abeliana. Por outro lado, ganhamos informação sobre o funtor $T(\mathcal{C}) \rightarrow \mathcal{C}$. A ideia de usar a regularidade do topos para obter informação de algo da forma $T(\mathcal{C})$ é também apresentada no Capítulo 8 de [BW85].

A diferença entre categorias abelianas e topos reside no comportamento do objeto inicial em uma categoria cartesianamente fechada como o topos, assim, se retiramos esse axioma da definição de topos obtemos o que se conhece por pretopos conseguimos um ambiente em que categorias abelianas e pretopos compartilham mais semelhanças, a saber, essas são as AT Categorias. Até onde sabemos, não existe pesquisa em AT Categorias, elas surgiram em uma discussão entre Vaughan Pratt e Peter J. Freyd justamente sobre as semelhanças entre topos e categorias abelianas e serve para satisfazer a curiosidade de como aproximar essas duas categorias, mas não são estruturas que aparecem de forma natural [nLa19].

Aproveitando que estamos fazendo comparações, vamos agrupar resultados parecidos entre a cohomologia de feixes de grupos abelianos e a cohomologia de feixes de um casal $(\mathcal{C}, J)$ através de uma tabela no mesmo espírito da anterior.

\begin{tabular}{l|l|l} 
& $\mathbf{A b}(\operatorname{Sh}(X))$ & $\mathbf{A b}(S h(\mathcal{C}, J))$ \\
\hline É categoria abeliana & Teorema 3.1.10 & Teorema 3.3.3 \\
Possui suficientes injetivos & Teorema 3.1.2 & Teorema 3.3.7 \\
Todo feixe flácido é acíclico & Proposição 3.2.9 & Proposição 3.3.12(i) \\
Cohomologia de Čech & Definição 3.4.3 & Definição 3.4.13
\end{tabular}

Ao contrário da anterior, essa tabela seria mais a tradução de um caso mais particular, $\mathbf{A b}(S h(X))$, para um caso mais geral, $\mathbf{A b}(S h(\mathcal{C}, J))$. Comparando teorema lado a lado veremos que não são exatamente os mesmo resultados quando estamos em $\mathbf{A b}(S h(\mathcal{C}, J))$ precisamos ser mais cuidados e as demonstrações exigem um nível de abstração maior. Por exemplo, a verificação de que flácido implica acíclico é para funtores distintos, enquanto para uma caso bastava trabalhar com o funtor $\Gamma(X,-)$ para o outro precisamos da imagem direta de um morfismo geométrico $f$ e utilizamos sequências espectrais para concluir o resultado. Há ainda outras construções conhecidas de cohomologia de feixes que encontram sua versão em $\mathbf{A b}(S h(\mathcal{C}, J))$ e que não tratamos aqui, uma delas é a resolução de Godement. [Joh14]. Isso nos leva a outro possível caminho a ser seguido: analisar para quais topos conseguimos manter as propriedades de $\operatorname{Ab}(S h(\mathcal{C}, J))$, em particular, aquelas que são interessantes para aplicar técnicas de Álgebra Homológica. Ou seja, para quais topos $\mathcal{E}$ 
conseguimos pelo menos garantir que $\mathbf{A b}(\mathcal{E})$ possui suficientes injetivos. Ainda, encontrar em quais topos de Grothendieck além do $S h(X)$ existe uma noção de feixe flácido definido de forma que $\mathscr{F}(X) \rightarrow \mathscr{F}(U)$ é sobrejetor. Investigações dessa natureza já têm sido feitas como pode ser visto em [Ble18].

Pensando nas aplicações, nesse trabalho vimos as relações de topos com teoria de cohomologia, mas as interações com teoria de homotopia têm sido mais pesquisadas por causa do conceito de $\infty$-topos, ver [Lur09]. Algumas ideias que conversam com teoria de homotopia também aparecem em [Joh14], através dos grupos fundamentais profinitos e categorias de Galois.

No exemplo 8.15(iii) de [Joh14] observa-se que a cohomologia do topos coincide com a cohomologia de Galois quando $G$ é um grupo profinitio e estamos na categoria dos $G$-sets contínuos com mapas $G$-equivariantes. Tendo em vista que a cohomologia de Galois é utilizada em Teoria dos Números - por exemplo, na demonstração do Teorema de Mordell-Weil [Sil09] - podemos dizer que a cohomologia de topos encontra uso em Teoria dos Números e seria uma tarefa interessante verificar em que outras situações teríamos aplicações úteis.

Temos bem conhecidas aplicações de topos de Grothendieck e diversas outras cohomologias (Étale e Nisnevich, para citar algumas) em Geometria Algébrica, porém, mais recentemente tem se utilizado os recursos da lógica interna do topos, [Ble18]. Nessa tese de doutorado, entitulada "Using the internal language of toposes in algebraic geometry", I. Blechschmidt usa que esquemas possuem um topos de Zariski associado e se aproveita da linguagem interna destes para resultados de Geometria Álgebrica. No Capítulo 3 de nossa Dissertação pincelamos alguns argumentos da linguagem interna do topos para simplificar a demonstração de que $\mathbf{A b}(\mathcal{E})$ é uma categoria abeliana, mas após sabermos que $\mathbf{A b}(\mathcal{E})$ é abeliana para um topos $\mathcal{E}$ poderíamos passar a usar também a técnica dos pseudolementos (Definição 1.7.1). No Capítulo 1 usamos os pseudoelementos para demonstrar o Lema da Serpente em categorias abelianas, seria interessante ver se novos resultados ou simplificações de resultados já conhecidos surgem se unirmos os recursos dos pseudoelementos e da linguagem interna do topos quando nos aprofundamos na estrutura de $\mathbf{A b}(\mathcal{E})$.

Pontuamos ainda que as ideias desenvolvidas para a categoria $\operatorname{Ab}(\mathcal{E})$ no Capítulo 3 pode ser reproduzido para $R-\operatorname{Mod}(\mathcal{E}), \operatorname{com} R$ um objeto anel na categoria $\mathcal{E}$. Não verificamos, mas deve ser verdade que $R$-Mod $(\mathcal{E})$ é uma categoria abeliana com suficientes injetivos quando $\mathcal{E}$ é um topos de Grothendieck e a demonstração deve ser bastante similiar ao caso $\mathbf{A b}(\mathcal{E})$. As perguntas que fizemos nesse Capítulo para $\mathbf{A b}(\mathcal{E})$ também podem ter refeitas para $R-\operatorname{Mod}(\mathcal{E})$, mas há outras:

- Caso $\mathcal{E}$ seja um topos com recursos categoriais suficientes (o topos de Grothendieck é um exemplo disso pelas propriedades que possui, segundo o Teorema de Giraud), conseguiriamos refazer as provas de teoremas de representação de categorias abelianas internas a um topos mas agora como subcategorias de $R$-Mod $(\mathcal{E})$ ?

- Seria possível descrever a noção de categoria abeliana de forma independente do topos base, isto é, poderíamos utilizar a ideia de diagramas internos para formular o que é uma categoria abeliana em um topos $\mathcal{E}$ ? Aqui estamos usando a noção de diagrama interno introduzida na Definição 2.4.25.

Não está claro ao longo do texto, em alguns momentos ao longo do trabalho os diagramas internos discretamente pareciam se relacionar com a categoria $\mathbf{A b}(\mathcal{E})$, a última pergunta enunciada acima deve ser o que melhor expõe o tipo de relação que detectamos e esperamos respondê-la no futuro.

Essas considerações finais foram expandidas na versão corrigida do texto graças às observações e sugestões dos membros titulares da banca, mais uma vez agradeço aos Professores Peter Arndt e Walter Pedra por acrescentarem novas perspectivas e mais possibilidades para pesquisas futuras. 


\section{Capítulo 5}

\section{Apêndice}

Deixamos esse espaço para listar alguns resultados de Teoria de Categorias que foram utilizados pelo menos uma vez ao longo da Dissertação. Não vamos demonstrá-los, são resultados bastante conhecidos e facilmente encontrados na literatura. As referências clássicas são [ML98] e [Bor94a].

Proposição 5.0.1 Sejam $f$ e g morfismos em uma categoria qualquer.

1. Se $f \circ g$ é mono, então g é mono.

2. Se $f \circ g$ é epi, então $f$ é epi.

Lema 5.0.2 (Lema do Pullback) Considere o seguinte diagrama comutativo<smiles></smiles>

Suponha que o quadrado da direita é um diagrama de pullback. Então o diagrama da esquerda é um diagrama de pullback se, e somente se, o retângulo inteiro é um diagrama de pullback.

Proposição 5.0.3 Seja $\mathcal{C}$ uma categoria. São equivalentes:

(i) $\mathcal{C}$ possui todos os limites finitos.

(ii) $\mathcal{C}$ possui produtos binários, equalizadores e objeto terminal.

(iii) $\mathcal{C}$ possui pullback e objeto terminal. 


\section{Referências Bibliográficas}

[Bar71] Michael Barr. Exact categories. Em Exact categories and categories of sheaves, páginas 1-120. Springer, 1971. 126

[Bel08] John L. Bell. Toposes and local set theories: an introduction. Courier Corporation, 2008. 43

[Bla83] Andreas Blass. Cohomology detects failures of the axiom of choice. Transactions of the American Mathematical Society, 279(1):257-269, 1983. 125

[Ble18] Ingo Blechschmidt. Using the internal language of toposes in algebraic geometry, 2018. 127

[Bor94a] Francis Borceux. Handbook of Categorical Algebra: Volume 1, Basic Category Theory. Cambridge Textbooks in Linguis. Cambridge University Press, 1994. 7, 44, 129

[Bor94b] Francis Borceux. Handbook of Categorical Algebra: Volume 2, Categories and Structures. Cambridge Studies in Philosoph. Cambridge University Press, 1994. $\mathrm{x}, 1,2,13,36,39,40,93,125,126$

[Bor94c] Francis Borceux. Handbook of Categorical Algebra: Volume 3, Sheaf Theory. Encyclopedia of Mathematics and its Applications. Cambridge University Press, 1994. $1,44,78,79,88,92$

[Bre13] Glen E. Bredon. Topology and geometry, volume 139. Springer Science \& Business Media, 2013. 18

[BW85] Michael Barr e Charles Wells. Toposes, triples, and theories. Grundlehren der mathematischen Wissenschaften. Springer-Verlag, 1985. 96, 126

[CE99] Henri Cartan e Samuel Eilenberg. Homological Algebra. Princeton landmarks in mathematics and physics. Princeton University Press, 1999. ix, 1

[Flo13] Cecilia Flori. A First Course in Topos Quantum Theory. Lecture Notes in Physics. Springer Berlin Heidelberg, 2013. 43

[Fre64] Peter Freyd. Abelian categories: an introduction to the theory of functors. Harper's series in modern mathematics. Harper \& Row, 1964. 1, 33

[GM02] Sergei I. Gelfand e Yuri I. Manin. Methods of Homological Algebra. Springer Monographs in Mathematics. Springer Berlin Heidelberg, 2002. 1, 16

[Gro57] Alexander Grothendieck. Sur quelques points d'algèbre homologique, i. Tohoku Math. J. (2), 9(2):119-221, 1957. ix, 1, 30, 31, 34, 36, 108

[Har77] Robin Hartshorne. Algebraic Geometry. Encyclopaedia of mathematical sciences. Springer, 1977. 20, 112

[HS71] Peter J. Hilton e Urs Stammbach. A course in homological algebra. Graduate texts in mathematics. Springer-Verlag, 1971. 1, 26, 32, 34, 35 
[Ive84] Birger Iversen. Cohomology of Sheaves. Aarhus Universitet, Matematisk Institut: Lecture notes series. Inst., Univ., 1984. 50, 95, 99, 100

[Joh02a] Peter T. Johnstone. Sketches of an Elephant: A Topos theory compendium. Oxford logic guides. Oxford Univ. Press, New York, NY, 2002. 43, 70

[Joh02b] Peter T. Johnstone. Sketches of an Elephant: A Topos Theory Compendium. Number v. 2 in Oxford Logic Guides. Clarendon Press, 2002. 92, 96

[Joh14] Peter T. Johnstone. Topos Theory. Dover Books on Mathematics. Dover Publications, 2014. x, 43, 61, 66, 71, 79, 95, 103, 105, 106, 107, 109, 112, 125, 126, 127

[Joy08] André Joyal. The theory of quasi-categories and its applications, 2008. 112

[Lin69] Fred E. J. Linton. Coequalizers in categories of algebras. Em Seminar on Triples and Categorical Homology Theory, páginas 75-90. Springer Berlin Heidelberg, 1969. 107

[Lur09] Jacob. Lurie. Higher Topos Theory (AM-170), volume 189. Princeton University Press, 2009. 127

[McC01] John McCleary. A User's Guide to Spectral Sequences. A User's Guide to Spectral Sequences. Cambridge University Press, 2001. 34

[Mc190] Colin Mclarty. The uses and abuses of the history of topos theory. British Journal for the Philosophy of Science, 41(3):351-375, 1990. 43, 65

[McL96] Colin McLarty. Elementary Categories, Elementary Toposes. Oxford University Press, Inc., New York, NY, USA, 1996. 2, 92

[ML98] Saunders Mac Lane. Categories for the Working Mathematician. Graduate Texts in Mathematics. Springer New York, 1998. 1, 50, 60, 129

[MLM94] Saunders Mac Lane e Ieke Moerdijk. Sheaves in Geometry and Logic: A First Introduction to Topos Theory. Universitext. Springer New York, 1994. x, 43, 44, $49,50,55,61,63,70,72,78,79,82,85,95,97,100$

[nLa19] nLab authors. AT category. http://ncatlab.org/nlab/show/AT\%20category, Janeiro 2019. Revision 23. 126

[Pic02] Rogerio C. Picanço. Álgebra homologica de feixes. 2002. 20

[Sil09] Joseph H Silverman. The arithmetic of elliptic curves, volume 106. Springer Science \& Business Media, 2009. 127

[Şte81] Alexandru Ştefănescu. The category of abelian groups in a topos. Bulletin mathématique de la Société des Sciences Mathématiques de la République Socialiste de Roumanie, 25 (73)(2):179-189, 1981. 107

[Ten75] Barry R. Tennison. Sheaf Theory. Cambridge Monographs on Physic. Cambridge University Press, 1975. 45, 46, 95, 97

[Wei95] Charles A. Weibel. An Introduction to Homological Algebra. Cambridge Studies in Advanced Mathematics. Cambridge University Press, 1995. x, 1, 16, 20, 34 